This document is downloaded from DR-NTU (https://dr.ntu.edu.sg) Nanyang Technological University, Singapore.

Micromechanical models based on hypersingular integro-differential equations for analyzing weak interfaces between dissimilar orthotropic materials

Wang, Xue

2015

https://hdl.handle.net/10356/65541

https://doi.org/10.32657/10356/65541 


\section{MICROMECHANICAL MODELS BASED ON HYPERSINGULAR INTEGRO-DIFFERENTIAL EQUATIONS FOR ANALYZING WEAK INTERFACES BETWEEN DISSIMILAR ORTHOTROPIC MATERIALS}

\section{WANG XUE}

A thesis submitted to Nanyang Technological University in partial fulfilment of the requirement for the degree of Doctor of Philosophy in School of Mechanical and Aerospace Engineering 



\section{Abstract}

This thesis is concerned with the development and formulation of micromechanical models for estimating the effective stiffness coefficients of a microscopically damaged interface between dissimilar orthotropic materials under antiplane and inplane deformations. The effective stiffness coefficients are important parameters in the macro-level spring-like model for a weak interface.

The microscopically damaged interface is modeled as containing a periodic array of micro-cracks. Three different micromechanical models are proposed for the micro-cracked interface.

The first model is the so called three-phase model which highly simplifies a period interval of the interface into three parts: (i) a representative microcrack, (ii) perfectly bonded parts and (iii) effective regions with an unknown stiffness. The only details of the original micro-cracked interface captured in the three-phase models are the density of the micro-cracks and the average micro-crack length which is the length of the representative micro-crack.

In the second model, all the micro-cracks are of the same length and are evenly distributed throughout the interface. Like the three-phase model, this model is a highly simplified one having only the density and the average length of the micro-cracks as micro-details of the micro-cracked interface. As may be expected, the values of the effective stiffness coefficients calculated using the second model are close to the ones predicted by the three-phase model. 
The third model is a more realistic one in which a period interval of the interface contains a large number of randomly generated micro-cracks. It is used to analyze statistically the effective stiffness coefficients of the microcracked interface. In addition to the density and average length of the microcracks, the statistical variations in the micro-crack length and the random positions of the micro-cracks are captured in such a micromechanical-statistical approach.

The interfacial conditions in all the three micromechanical models are formulated in terms of hypersingular integro-differential equations. The displacement jumps across the damaged parts of the interface, which appear directly as unknown functions in the hypersingular integro-differential equations, are needed to estimate the effective stiffness coefficients of the micro-cracked interface.

The micromechanical models above are used to estimate the effective stiffness coefficients of micro-cracked interfaces for each of the following problems: (i) an interface between two dissimilar half-spaces, (ii) an interface between a thin elastic layer and an elastic half-space, and (iii) a pair of parallel interfaces in an elastic trimaterial. Each of the problems under consideration is formulated in terms of hypersingular integro-differential equations. Numerical procedures for solving these hypersingular integro-differential equations and estimating the effective stiffness coefficients are outlined.

The numerical results obtained from the micromechanical models demonstrate that the effective stiffness coefficients are influenced by the densities of the micro-cracks on the interfaces, the elastic moduli and the geometries of the materials. Numerical values of the effective stiffness coefficients are obtained for parametric studies for specific cases of the problems under consideration. The effects of various parameters, such as the micro-crack density and the 


\section{Abstract}

variations in the micro-crack length, on the effective stiffness coefficients are examined in detail. 



\section{Acknowledgments}

Having completed the writing of this thesis, I would like to acknowledge Nanyang Technological University for financial support in the form of a Nanyang Engineering Doctoral Scholarship and humbly thank many people who had helped me one way or another in my research work.

I would like to express my most sincere gratitude to my supervisor Professor Ang Whye Teong for the consistent guidance he had given me throughout my entire Ph.D. studies. I have benefited from his expertise in the boundary element method, general knowledge in mathematics and sharp attention to details in research matters. His advice and encouragement had helped me to overcome difficulties encountered during the research and writing of this thesis.

I would also like to express my deep appreciation to my co-supervisor Professor Fan Hui. He had provided me with valuable suggestions and comments on the micromechanical aspects of this research project. I am impressed by his academic insights and serious attitude to research work.

Special thanks are due to all my seniors for sharing their research experience with me and always being there when I requested their help.

Lastly but not least, I am eternally grateful for the constant encouragement, care and support from my beloved parents and loving fiancé. 



\section{Contents}

page

Abstract

Acknowledgments $\quad$ v

Contents vii

List of Figures $\quad$ xiii

List of Tables $\quad$ xix

1 General Introduction 1

1.1 Motivation .................... 1

1.2 Micromechanical Models for Estimation of Effective Properties . 3

1.3 Hypersingular Integral Approach for Crack Problems . . . . . . 9

1.4 The Present Thesis . . . . . . . . . . . . . . . . . . . . 11

1.4.1 Research Objective and Scope . . . . . . . . . 11

1.4.2 Overview of Remaining Chapters ... . . . . . . 11

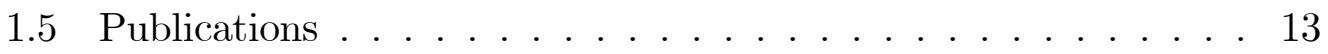

2 Mathematical Preliminaries $\quad 15$

2.1 Introduction . . . . . . . . . . . . . . . 15 
2.2 Basic Equations of Elasticity . . . . . . . . . . . . . . . 15

2.2.1 Antiplane Deformations _. . . . . . . . . . . . 17

2.2 .2 Inplane Deformations . . . . . . . . . . . . . . 18

2.3 Boundary Integral Equations in Elasticity _ . . . . . . . . . . 20

2.3.1 Antiplane Deformations . . . . . . . . . . . . 20

2.3.2 Inplane Deformations . . . . . . . . . . . . . . . . . 22

2.4 Special Green's Functions for Perfect Interfaces _ . . . . . . . 23

2.4.1 Antiplane Green's Function . . . . . . . . . . . . . 23

2.4 .2 Inplane Green's Function . . . . . . . . . . . . . . . . 25

2.5 Hadamard Finite-part Integrals _ . . . . . . . . . . . . 27

2.6 Summation Formulae Involving Digamma and Trigamma Func-

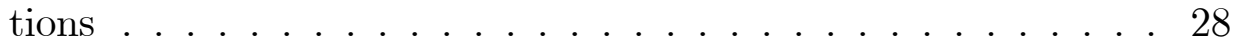

2.7 Chi-square $\left(\chi^{2}\right)$ Distribution $\ldots \ldots \ldots \ldots$

3 Weak Interfaces between Two Orthotropic Half-spaces 33

3.1 Introduction . . . . . . . . . . . . . . . . . . . . . 33

3.2 A Micro-cracked Interface . . . . . . . . . . . . . . . . 34

3.3 Hypersingular Integro-differential Equations for Interfacial Micro-

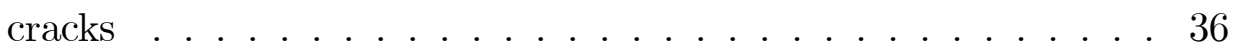

3.3.1 Antiplane Deformations . . . . . . . . . . . . 37

3.3 .2 Inplane Deformations . . . . . . . . . . . . . . . . 42

3.4 Micromechanical Models for Estimating Effective Stiffness _ . . 44

3.4.1 Three-phase Model . . . . . . . . . . . . . . . . . . . . 45

3.4.2 Periodic Model Involving Evenly Distributed Micro-cracks of Equal Length . . . . . . . . . . . . . . . . 48

3.4.3 Micromechanical-statistical Model . . . . . . . . . . . . . 50

3.5 Numerical Procedures . . . . . . . . . . . . . . . . . . . 53 
3.5.1 Three-phase Model . . . . . . . . . . . . . . . . . . 53

3.5.2 Periodic Model Involving Evenly Distributed Micro-cracks of Equal Length . . . . . . . . . . . . . . . . 60

3.5.3 Micromechanical-statistical Model . . . . . . . . . . . . . 62

3.5.4 Numerical Values of Effective Stiffness Coefficients . . . . 65

3.6 Numerical Comparison of the Three-Phase and the Periodic Models . . . . . . . . . . . . . . . . . . . 66 66

3.6.1 Antiplane Deformations . . . . . . . . . . . . 66

3.6.2 Inplane Deformations . . . . . . . . . . . . . . . . 68

3.7 Micromechanical-statistical Simulations . . . . . . . . . . . . 70

3.7.1 Number of Micro-cracks for Homogenizing the Interface . 71

3.7.2 Effect of the Micro-crack Length Distribution on the Effective Stiffness Coefficients . . . . . . . . . . . . 73

3.7.3 Effect of the Micro-crack Tip Gap Distribution on the Effective Stiffness Coefficients . . . . . . . . . . . 76

3.7.4 Case Studies Using Specific Orthotropic Materials for Inplane Deformations . . . . . . . . . . . . . . . 78

3.7.5 Isotropic Bimaterial Subject to Inplane Deformations . . 80 3.8 Summary . . . . . . . . . . . . . . . . . 83

4 Weak Interfaces between Thin Orthotropic Layers and Orthotropic Half-spaces $\quad 85$

4.1 Introduction . . . . . . . . . . . . . . 85

4.2 Micromechanical Models . . . . . . . . . . . . . . . . . 86

4.2.1 Three-phase Model . . . . . . . . . . . . . . . . . . . 88

4.2.2 Periodic Model Involving Evenly Distributed Micro-cracks of Equal Length . . . . . . . . . . . . . . . . . . . . 89 
4.2.3 Micromechanical-statistical Model . . . . . . . . . 89

4.3 Hypersingular Boundary Integro-differential Equations ... . 90

4.3.1 Three-phase Model . . . . . . . . . . . . . . 91

4.3.2 Periodic Model Involving Evenly Distributed Micro-cracks of Equal Length . . . . . . . . . . . . . . . . . 98

4.3.3 Micromechanical-statistical Model . . . . . . . . . . . 100

4.4 Numerical Procedures . . . . . . . . . . . . . . . . 103

4.4.1 Three-phase Model . . . . . . . . . . . . . . . . . 103

4.4.2 Periodic Model Involving Evenly Distributed Micro-cracks of Equal Length . . . . . . . . . . . . . . . 105

4.4.3 Micromechanical-statistical Model . . . . . . . . . . 106

4.4.4 Numerical Values of Effective Stiffness Coefficients . . . . 106

4.5 Numerical Comparison between the Three-phase and the Periodic Models . . . . . . . . . . . . . . . . 107 4.5.1 Antiplane Deformations . . . . . . . . . . 107

4.5.2 Inplane Deformations . . . . . . . . . . . . . . . . 110

4.6 Micromechanical-statistical Simulations . . . . . . . . . . . . . . 112

4.6.1 Number of Micro-cracks for Homogenizing the Interface . 113

4.6.2 Influence of the Micro-crack Length Distribution . . . . . 115

4.6.3 Case Studies Using Specific Orthotropic Materials for Inplane Deformations . . . . . . . . . . . . . . . 118

4.6.4 Isotropic Bimaterial Subject to Inplane Deformations . . 120

4.6.5 Parametric Studies for Antiplane Deformations . . . . . 124

4.7 Summary . . . . . . . . . . . . . . . . . . 129

5 Interaction of a Pair of Parallel Weak Interfaces 133

5.1 Introduction ........................ 133 
5.2 A Pair of Micro-cracked Interfaces . . . . . . . . . . . . . . 134

5.3 Boundary Value Problems . . . . . . . . . . . . . . . . 137

5.3.1 Antiplane Deformations . . . . . . . . . . . . 138

5.3.2 Inplane Deformations . . . . . . . . . . . . . . . . 139

5.4 Hypersingular Boundary Integro-differential Equations . . . . 140

5.4 .1 Antiplane Deformations . . . . . . . . . . . . . 140

5.4 .2 Inplane Deformations . . . . . . . . . . . . . . . . . 143

5.5 Numerical Procedures . . . . . . . . . . . . . . . . . . 148

5.5.1 Antiplane Deformations . . . . . . . . . . . . . . 149

5.5 .2 Inplane Deformations . . . . . . . . . . . . . . . 151

5.6 Numerical Results for Antiplane Deformations . . . . . . . . . 153

5.6.1 A Comparison of Two Micro-models . . . . . . . . . . 153

5.6.2 Micromechanical-statistical Simulations . . . . . . . . . 161

5.7 Numerical Results for Inplane Deformations . . . . . . . . . 167

5.7.1 A Perfect Interface and a Micro-cracked Interface . . . 168

5.7.2 A Particular Periodic Model for a Pair of Micro-cracked Interfaces . . . . . . . . . . . . . . . . . 172

5.7.3 Micromechanical-statistical Simulations . . . . . . . . 180

5.8 Summary . . . . . . . . . . . . . . . . . . . . . 182

6 Research Contributions and Extensions 185

6.1 Summary of Contributions . . . . . . . . . . . . . 185

6.2 Research Extensions _. . . . . . . . . . . . . . . . . 187

$\begin{array}{ll}\text { Bibliography } & 189\end{array}$ 



\section{List of Figures}

1.1 A sketch of the microscopically damaged interface and the springlike interface. . . . . . . . . . . . . . . . 2

1.2 A sketch of the multiphase composite material and the equivalent homogeneous solid. . . . . . . . . . . . . . . 4

1.3 Three micromechanical approaches for modeling the composite material in Figure 1.2: (a) the three-phase model, (b) periodically repeated inclusions embedded in the matrix, and (c) randomly generated microscopic inclusions embedded in the matrix. 5

1.4 A microscopically damaged plane interface between two dissimilar materials. . . . . . . . . . . . . . . 7

1.5 Three micromechanical models for the microscopically imperfect interface: (a) three-phase model, (b) periodically repeated micro-cracks, and (c) randomly positioned micro-cracks of randomly generated sizes. . . . . . . . . . . . . . 8

2.1 A sketch of two dissimilar orthotropic half-spaces perfectly bonded along $x_{2}=0 . \ldots \ldots \ldots \ldots \ldots \ldots \ldots$

2.2 Relative frequency distribution of the micro-crack lengths generated by using the $\chi^{2}$ distribution of degrees of freedom 5, 10 and 25. A unit length of the micro-crack is denoted by $u$. . . . 30 
3.1 A sketch of a period length of the micro-cracked interface between two dissimilar half-spaces. . . . . . . . . . . . . . . . 35

3.2 A geometrical sketch of the three-phase model for the microcracked interface. . . . . . . . . . . . . . . . . 46

3.3 A sketch of the periodic model. . . . . . . . . . . . . . . 49

3.4 Plots of $a\left(\beta^{(1)}+\beta^{(2)}\right) k_{33} /\left(2 \beta^{(1)} \beta^{(2)}\right)$ against $\rho \ldots \ldots \ldots \ldots 7$

3.5 Plots of $a k_{11} / C_{66}^{(2)}$ and $a k_{22} / C_{66}^{(2)}$ against $\rho \ldots \ldots \ldots 9$

3.6 Scatter plots and mean values of the non-dimensionalized effective stiffness coefficients against $M \ldots \ldots \ldots \ldots$. . . . 72

3.7 Plots of mean values $\widehat{K}_{11}, \widehat{K}_{22}$ and $\widehat{K}_{33}$ against the damage ratio $\rho$, for micro-crack length generated by the $\chi^{2}$ distribution of degrees of freedom 5, 10 and 25. Values predicted by the three-phase model are also plotted. . . . . . . . . . . . . . 74

3.8 Plots of the mean values $\widehat{K}_{33}$ against the damage ratio $\rho$, for distributions of micro-crack tip gap generated using the $\chi^{2}$ distribution of degrees of freedom 5, 10 and 25. Also included are the corresponding values predicted by the three-phase model. . . 77

3.9 Plots of $\widehat{K}_{11}$ and $\widehat{K}_{22}$ against $\rho$ for selected materials in the upper half-space. . . . . . . . . . . . . . . . . 79

3.10 Plots of $\widehat{K}_{11}$ against $\beta$ for selected values of $\rho \ldots \ldots$. . . 82

4.1 A sketch of the micro-cracked interface between the thin orthotropic layer and the orthotropic half-space. Note that $L$ is the period length of the periodically repeated micro-cracked interface and $\left(a^{(m)}, 0\right)$ and $\left(b^{(m)}, 0\right)$ are respectively the left and the right tips of the $m$-th micro-crack. . . . . . . . . 87 
4.2 A sketch of the three-phase model for the micro-cracked interface between the thin orthotropic layer and the orthotropic halfspace. The interface comprises three distinct parts as described in Subsection 3.4.1. . . . . . . . . . . . . . . . . . . . 88

4.3 A sketch of the periodic model for the micro-cracked interface between the thin orthotropic layer and the orthotropic halfspace. The interface contains a periodic array of evenly distributed micro-cracks of equal length $2 a \ldots \ldots$. . . . . . 89

4.4 Plots of $a k_{33} / G^{(2)}$ against $\rho$ for the shear modulus ratio $G^{(1)} / G^{(2)}=$ 1 and selected values of $a / h \ldots \ldots \ldots \ldots$

4.5 Plots of $a k_{33} / G^{(2)}$ against $a / h$ for the damage ratio $\rho=0.5$ and selected values of the shear modulus ratio $G^{(1)} / G^{(2)} \ldots \ldots \ldots 109$

4.6 Plots of $a k_{11} / C_{66}^{(2)}$ and $a k_{22} / C_{66}^{(2)}$ against $a / h$ for selected values

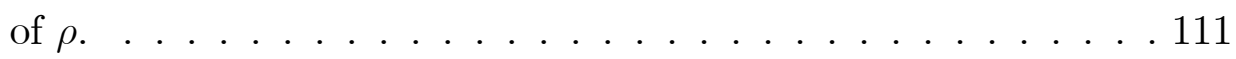

4.7 Scatter plots and mean values of the non-dimensionalized effective stiffness against $M . \ldots \ldots \ldots$. . . . . . . . . 114

4.8 Plots of $\widehat{K}_{11}$ and $\widehat{K}_{22}$ against $\widehat{a} / h$ for $\rho=0.5$ and cases where the micro-crack length follows the $\chi^{2}(5), \chi^{2}(10)$ and $\chi^{2}(25)$ distributions. Also given are plots of the corresponding nondimensionalized stiffness coefficients computed by using the threephase model. . . . . . . . . . . . . . . . . . 116

4.9 Plots of $\widehat{K}_{33}$ against $\widehat{a} / h$ for $\rho=0.5$, selected values of $G^{(1)} / G^{(2)}$ and cases where the micro-crack length follows the $\chi^{2}(5), \chi^{2}(10)$ and $\chi^{2}(25)$ distributions. Also given are plots of the corresponding non-dimensionalized stiffness coefficients computed by using the three-phase model. . . . . . . . . . . . . . . . . . . 117

4.10 Plots of $\widehat{K}_{11}$ and $\widehat{K}_{22}$ against $\widehat{a} / h$ for selected thin layer materials. 119 


\section{Micromechanical Models for Weak Interfaces}

4.11 Plots of $\widehat{K}_{11}$ for isotropic bimaterial against $\log _{10}\left(E^{(1)} / E^{(2)}\right)$ for selected values of $\widehat{a} / h, \rho=0.5$ and $\nu=0.25 \ldots . . . . . .123$

4.12 Plots of $\widehat{K}_{11}$ for isotropic bimaterial against $\nu$ for selected values of $E^{(1)} / E^{(2)}, \rho=0.5$ and $\widehat{a} / h=1 \ldots \ldots . \ldots . . . \ldots 124$

4.13 Plots of $\widehat{K}_{33}$ against $\rho$ for selected values of $\widehat{a} / h$ and $G^{(1)} / G^{(2)}$. 126

4.14 Plots of $\widehat{K}_{33}$ against $\widehat{a} / h$ for selected values of $G^{(1)} / G^{(2)}$ and $\rho .127$

4.15 Plots of $\widehat{K}_{33}$ against $\log _{10}\left(G^{(1)} / G^{(2)}\right)$ for $\rho=0.5$ and selected values of $\widehat{a} / h . \ldots \ldots \ldots \ldots$

5.1 A sketch of the geometry of the trimaterial for $M=3 . \quad \ldots 136$

5.2 A geometrical sketch of the periodic model for interfaces I and II.154

5.3 A geometrical sketch of the three-phase model for interfaces I and II. . . . . . . . . . . . . . . . . . . . . . . 155

5.4 Plots of $a_{\mathrm{I}} k_{33 \mathrm{I}} / G^{(2)}$ against $a_{\mathrm{I}} / h$ for $G^{(1)} / G^{(2)}=G^{(3)} / G^{(2)}=2$ and $\rho_{\mathrm{I}}=\rho_{\mathrm{II}}=0.3,0.5$ and $0.7 \ldots \ldots \ldots 1$

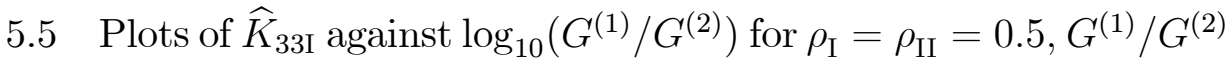
$=G^{(3)} / G^{(2)}$ and selected values of $\widehat{a}_{\mathrm{I}} / h \ldots \ldots . \ldots 163$

5.6 Plots of $\widehat{K}_{33 \mathrm{I}}$ against $\log _{10}\left(G^{(1)} / G^{(2)}\right)$ for $\widehat{a}_{\mathrm{I}} / h=1, G^{(1)} / G^{(2)}$ $=G^{(3)} / G^{(2)}, \rho_{\mathrm{I}}=\rho_{\mathrm{II}}=0.3,0.5$ and $0.7 \ldots \ldots . \ldots . \ldots 164$

5.7 Plots of $\widehat{K}_{33 \mathrm{I}}$ against $\widehat{a}_{\mathrm{I}} / h$ for $G^{(1)} / G^{(2)}=G^{(3)} / G^{(2)}=10, \rho_{\mathrm{I}}=$ $\rho_{\mathrm{II}}=0.4,0.5$ and $0.6 \ldots \ldots \ldots \ldots$. . . . . . . . . . . . . . . . . .

5.8 Plots of $\widehat{K}_{33 \mathrm{I}}$ and $\widehat{K}_{33 \mathrm{II}}$ against $\rho_{\mathrm{II}}$ for $\rho_{\mathrm{I}}=0.5, G^{(1)} / G^{(2)}=$ $G^{(3)} / G^{(2)}=10$ and selected values of $\widehat{a}_{\mathrm{I}} / h$. . . . . . . . 166

5.9 Plots of $a_{\mathrm{I}} k_{11 \mathrm{I}} / C_{66}^{(2)}$ and $a_{\mathrm{I}} k_{22 \mathrm{I}} / C_{66}^{(2)}$ against $a_{\mathrm{I}} / h$ for the cases where interface II is perfectly bonded with $\rho_{\mathrm{I}}=0.5$ and selected values of $\alpha$ 
5.10 Plots of $a_{\mathrm{I}} k_{11 \mathrm{I}} / C_{66}^{(2)}$ and $a_{\mathrm{I}} k_{22 \mathrm{I}} / C_{66}^{(2)}$ against $\log _{10} \alpha$ for the cases where interface II is perfectly bonded with $\rho_{\mathrm{I}}=0.5$ and selected values of $a_{\mathrm{I}} / h \ldots \ldots \ldots \ldots \ldots \ldots \ldots \ldots \ldots \ldots \ldots$

5.11 Plots of $a k_{1} / C_{66}$ and $a k_{2} / C_{66}$ against $a / h$ for $\rho=0.4,0.5$ and

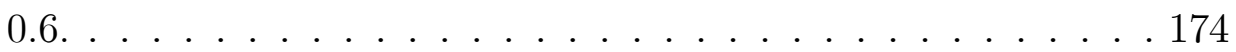

5.12 Plots of $\left(a_{\mathrm{I}}+a_{\mathrm{II}}\right) k_{11 \mathrm{I}} /\left(2 C_{66}\right)$ and $\left(a_{\mathrm{I}}+a_{\mathrm{II}}\right) k_{22 \mathrm{I}} /\left(2 C_{66}\right)$ against $\left(a_{\mathrm{I}}+a_{\mathrm{II}}\right) /(2 h)$ for selected values of $\rho_{\mathrm{II}} \ldots \ldots \ldots \ldots \ldots$

5.13 Plots of $\left(a_{\mathrm{I}}+a_{\mathrm{II}}\right) k_{11 \mathrm{II}} /\left(2 C_{66}\right)$ and $\left(a_{\mathrm{I}}+a_{\mathrm{II}}\right) k_{22 \mathrm{II}} /\left(2 C_{66}\right)$ against $\left(a_{\mathrm{I}}+a_{\mathrm{II}}\right) /(2 h)$ for selected values of $\rho_{\mathrm{II}} \ldots \ldots \ldots \ldots \ldots$

5.14 Plots of $a k_{1} / C_{66}^{(2)}$ and $a k_{2} / C_{66}^{(2)}$ against $a / h$ for the cases where martensite, graphite-epoxy and e-glass-epoxy are used as the material of the half-spaces. . . . . . . . . . . . . . . . . 179

5.15 Plots of the mean values $\widehat{K}_{i j \mathrm{I}}$ of the effective stiffness coefficients against $\widehat{a}_{\mathrm{I}} / h$ for $\rho_{\mathrm{I}}=\rho_{\mathrm{II}}=0.5 \ldots \ldots \ldots \ldots \ldots \ldots \ldots \ldots \ldots \ldots$ 



\section{List of Tables}

3.1 Elastic moduli (in GPa) for selected orthotropic materials. . . . 70

3.2 The mean $\widehat{K}_{33}$ and standard deviation $s_{33}$ of the antiplane nondimensionalized effective stiffness coefficient. . . . . . . . . 76 



\section{Chapter 1}

\section{General Introduction}

\subsection{Motivation}

Multilayered structures are nowadays widely used in many engineering applications such as in microelectromechanical devices (see, for example, Cui et al. [29], Michael and Kwok [58], Muralt [59] and Sun et al. [78]). The different layers in multilayered materials may be imperfectly bonded for various reasons. During the fabrication or usage of a multilayered material, micro-cracks may be formed in interfaces between layers due to mismatches in thermal and mechanical properties of materials (Nix [64]). Micro-roughness of surfaces may also give rise to interfacial imperfections (Hamidi et al. [42]).

Microscopically damaged interfaces are termed "soft" or "weak". For a more tractable analysis, such an imperfect interface may be modeled as a continuous distribution of springs characterized by a set of stiffness coefficients, as proposed in Benveniste and Miloh [13]. Refer to Figure 1.1. The tractions on the spring-like interface are linearly related to the jump in the displacements across opposite sides of the interface, that is, if the spring-like interface between materials 1 and 2 is denoted by $\Gamma$, then the interfacial conditions are given by

$$
\underline{\underline{\boldsymbol{\sigma}}}^{(1)} \cdot \underline{\mathbf{n}}=\underline{\underline{\boldsymbol{\sigma}}}^{(2)} \cdot \underline{\mathbf{n}}=\underline{\underline{\mathbf{k}}} \cdot\left(\underline{\mathbf{u}}^{(1)}-\underline{\mathbf{u}}^{(2)}\right) \text { on } \Gamma,
$$




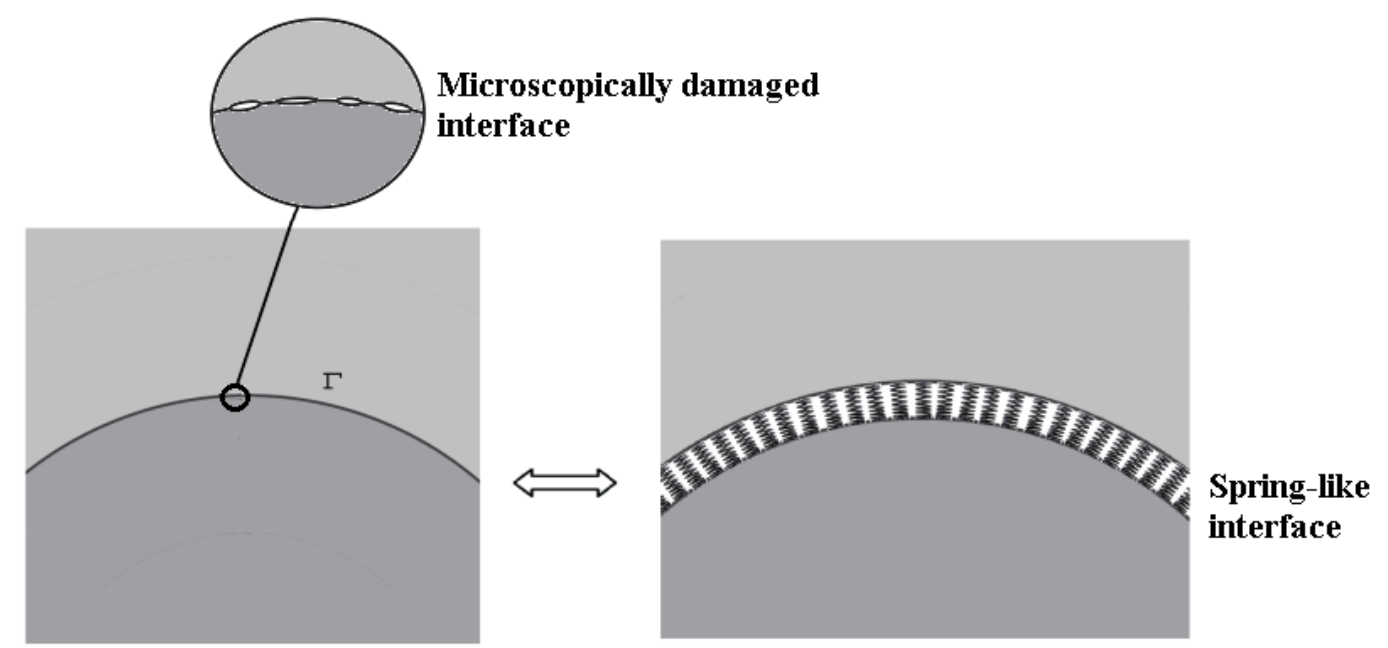

Figure 1.1: A sketch of the microscopically damaged interface and the springlike interface.

where $\underline{\mathbf{n}}$ is the unit normal vector to $\Gamma$ pointing into material $1, \underline{\mathbf{u}}^{(i)}$ and $\underline{\underline{\boldsymbol{\sigma}}}^{(i)}$ are respectively the displacement (first order tensor) and the stress (second order tensor) in material $i$ and $\underline{\underline{\mathbf{k}}}$ is the stiffness (second order tensor) of $\Gamma$.

The spring-like interface defined by (1.1) was used by many researchers for analyzing multilayered materials with imperfect weak interfaces. For example, Boström and Golub [18], Boström and Kvasha [19] and Ishii and Biwa [45] examined the propagation of ultrasonic waves in multilayered elastic materials with spring-like imperfect interfaces, and Fan and Wang [32], Sudak [76] and Wang [80] investigated the interaction between a dislocation and a spring-like interface. Earlier works involving spring-like interfaces may be found in Nagy [62], Pilarski and Rose [65], Rokhlin and Huang [67] and Rokhlin and Wang $[68]$.

Hitherto, not much research has been carried out on the micromechanical aspects of imperfect interfaces. To estimate the effective stiffness $\underline{\underline{\mathbf{k}}}$ in (1.1) for 
a microscopically damaged interface, Fan and Sze [31] presented a three-phase model in which the microscopically damaged interface was modeled using a representative micro-crack interacting with spring-like effective regions having an unknown stiffness. The micro-crack details captured in the highly simplified three-phase model were the average micro-crack length and the micro-crack density. In [31], the nonlinear boundary value problem for the three-phase model was solved numerically using a finite element technique which involved over 10000 elements.

Further research into the development of micromechanical models that take into account more realistic features of microscopically imperfect interfaces is needed. The micromechanical models must be based on computationally accurate and efficient numerical methods. An understanding on the micromechanical interaction of interfaces is essential in the analysis of engineering structures that are made up of extremely thin layers (such as micromechanical devices).

\subsection{Micromechanical Models for Estimation of Effective Properties}

The analysis of a microscopically heterogeneous material based on the continuum theory of elasticity is a subject of considerable interest. The heterogeneous material is modeled as a composite material which is made of an elastic matrix containing microscopic inhomogeneities such as holes or inclusions. At the macro level, the multiphase composite is regarded as a homogeneous material with an effective elastic property. A sketch of this is given in Figure 1.2 where the elastic moduli of the matrix and the $n$-th micro-inclusion are given by the second order tensors $\underline{\underline{\mathbf{C}}}^{(\text {matrix) }}$ and $\underline{\underline{\mathbf{C}}}^{(n)}$ respectively and the effective elastic moduli of the homogeneous material by $\underline{\underline{\mathbf{C}}}^{(0)}$. 
In the micromechanics literature, there are various micromechanical approaches, such as the Voigt approximation, the Reuss approximation, selfconsistent scheme, three-phase model and method of cells, for estimating the effective properties of multiphase composites (see, for example, Aboudi [2], Fan et al. [33], Li and Wang [54], Sertse and Yu [74], Xie and Fan [79], Yu and Tang [82] and Yu et al. [83]). Three of the main micromechanical approaches which are widely used in modeling the composite material in Figure 1.2 are illustrated in Figure 1.3 and briefly explained below.

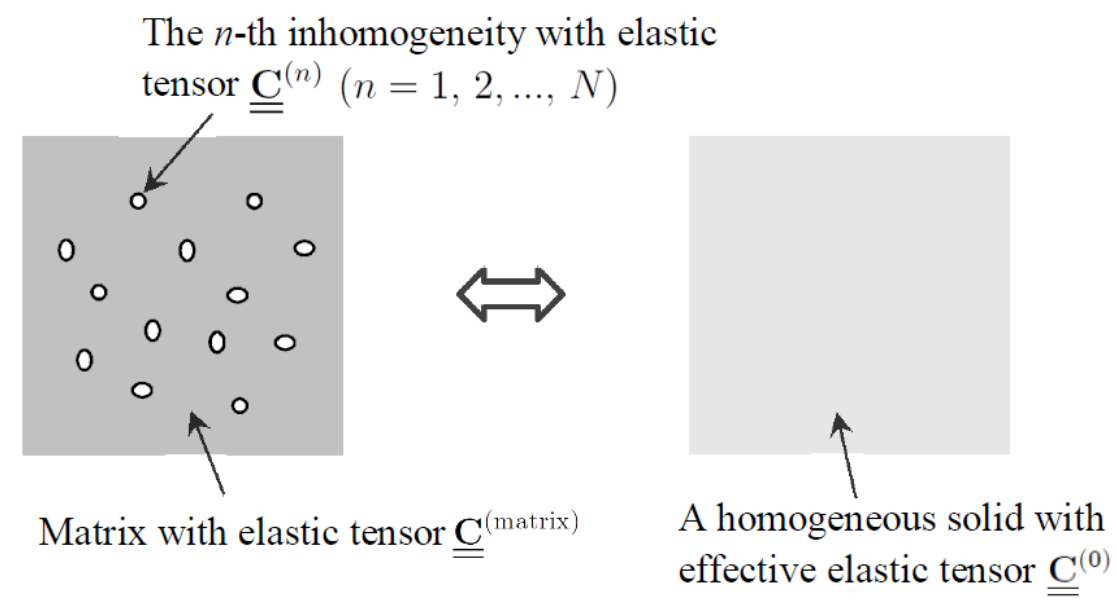

Figure 1.2: A sketch of the multiphase composite material and the equivalent homogeneous solid.

The three-phase model in Figure 1.3(a) simplifies the composite material to one consisting of three parts: a representative inclusion, a portion of the matrix surrounding the inclusion and a homogeneous effective region of infinite extent (Christensen and Lo [24]). The ratio of the area or volume of the inclusion to the matrix reflects the density of the microscopic inclusions in the matrix. The application of such a three-phase model in estimating the effective mechanical properties of composite materials is well documented in the literature (see, for 
example, Aboudi and Benveniste [4], Christensen [23], Colombini et al. [27] and Colombini and Maurer [28]).

(a)

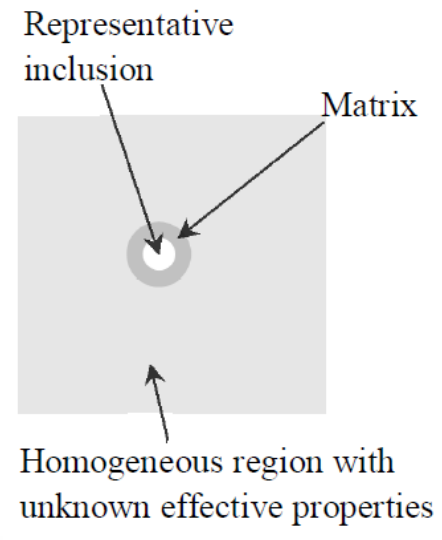

(b)

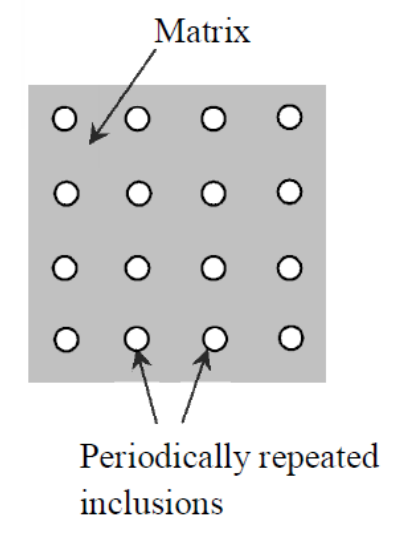

(c)

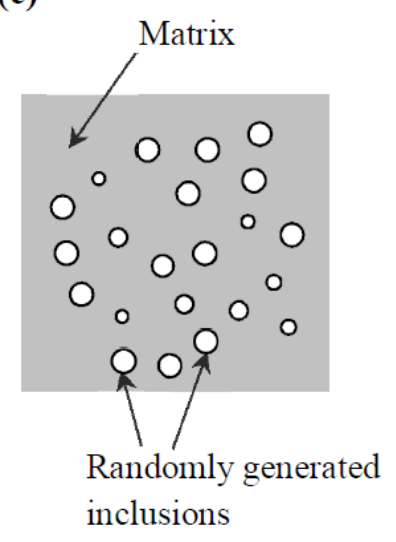

Figure 1.3: Three micromechanical approaches for modeling the composite material in Figure 1.2: (a) the three-phase model, (b) periodically repeated inclusions embedded in the matrix, and (c) randomly generated microscopic inclusions embedded in the matrix.

The approach in Figure 1.3(b) models the composite material as containing identical inclusions which are periodically embedded in the matrix. An example of such an approach is the generalized method of cells described in Aboudi 
$[1,3]$. It is widely used in estimating the effective properties of composite materials. In this method, the composite material under consideration is considered as containing periodically repeated unit cells. Each unit cell contains some characteristics of the multiphase material. In Kushch and Sevostianov [52], the unit cell consists of a continuous matrix with a spherical inclusion. Afonso and Ranalli [6], Berger et al. [15] and Guinovart-Díaz et al. [41] proposed periodic unit cells where each unit cell is a three-phase composite. Other works on the periodic unit cells for estimating the effective properties of multiphase materials may be found in Feng et al. [35, 36].

The approach illustrated in Figure 1.3(c) is a statistical one in which the composite material is modeled as containing randomly generated microscopic inclusions embedded in the matrix. To carry out such a statistical simulation, a selected number of micro-inclusions are randomly generated in a representative volume element which is much larger than each of the micro-inclusions and over which the macroscopic effective properties are uniform or homogenized. For example, to investigate the effective elastic properties of a rubber-toughened polymer, Danielsson et al. [30] modeled the polymer as containing periodically distributed representative volume elements, where a representative volume element contains a selected number of randomly generated rubber inclusions. Other related works involving statistical approaches for estimating effective properties may be found in Ju and Chen [49], Ju and Sun [50] and Segurado and LLorca [73].

As mentioned in Section 1.1, there is currently not much research work on the estimation of the effective stiffness of microscopically damaged interfaces. In Figure 1.4, we model a microscopically damaged plane interface between two dissimilar materials as containing micro-cracks. The parts of the interface outside the micro-cracks are perfectly bonded. If the effective stiffness 
$\underline{\underline{\mathbf{k}}}$ is a constant, that is, the microscopical damage is homogeneous over the interface, the micro-cracks may be taken to be periodically distributed over the whole interface. We adopt the three micromechanical approaches (Figure 1.3) for estimating effective properties of heterogeneous structures to analyze microscopically damaged interfaces between dissimilar materials as described below.

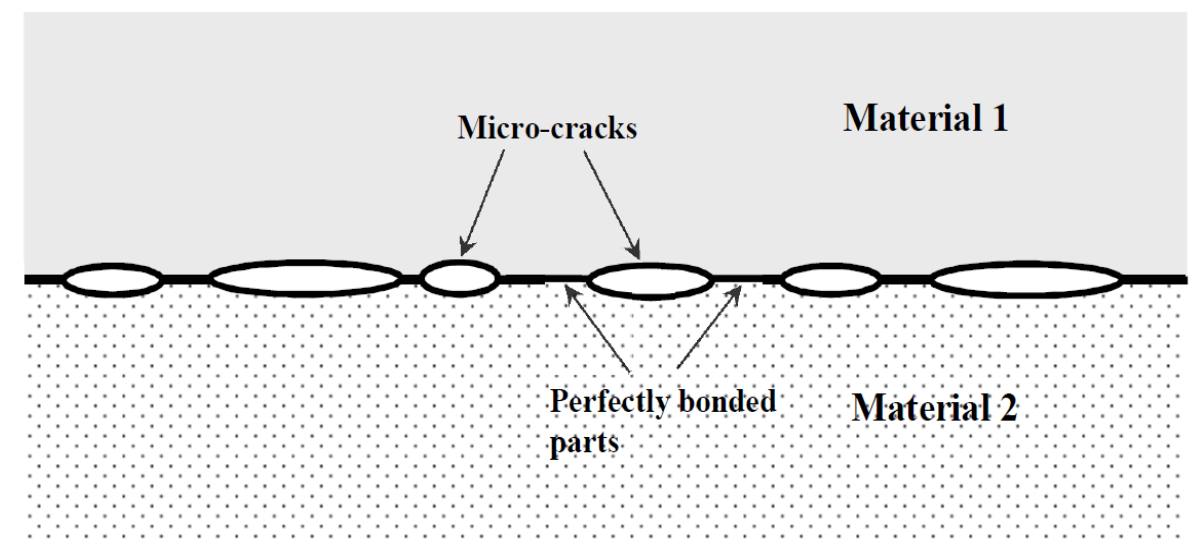

Figure 1.4: A microscopically damaged plane interface between two dissimilar materials.

In the three-phase model approach in Figure 1.5(a), a period interval of the interface consists of a representative micro-crack, perfectly bonded regions and effective regions with the effective stiffness yet to be determined. The length of each of the effective regions is usually taken to be much larger than the total length of the micro-crack and the perfectly bonded parts. A version of the three-phase model based on finite element analysis is given in Fan and Sze [31] for the interface between two rectangular strips.

In the second approach as sketched in Figure 1.5(b), we model a period interval of the interface as containing only one micro-crack. That is, microcracks of equal length are evenly distributed along the whole interface. As 
we shall see in later chapters, the effective stiffness estimated by using this approach is fairly close to the one given by the three-phase model in Figure $1.5(\mathrm{a})$.

(a)

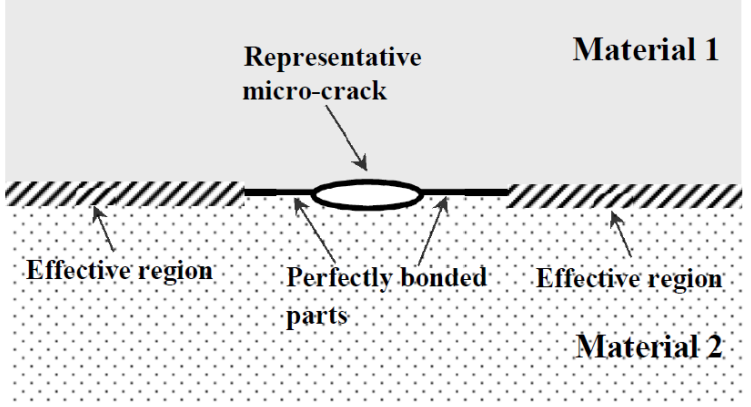

(b)

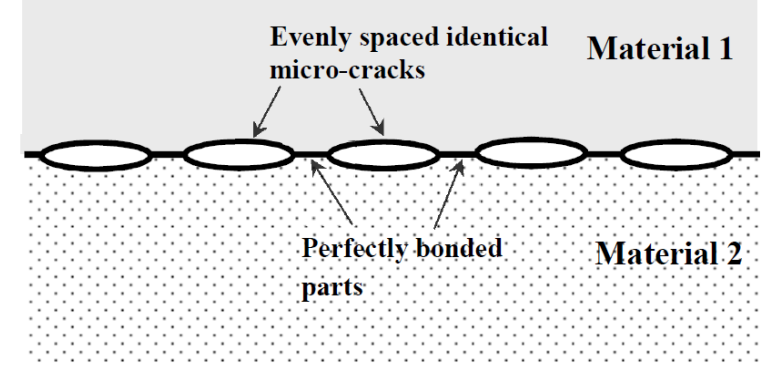

(c)

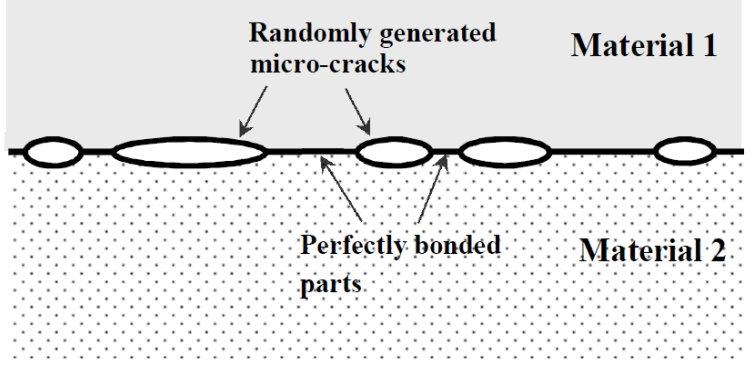

Figure 1.5: Three micromechanical models for the microscopically imperfect interface: (a) three-phase model, (b) periodically repeated micro-cracks, and (c) randomly positioned micro-cracks of randomly generated sizes.

The only details of the micro-cracked interface captured by the first two micromechanical approaches are the average length and the density of the in- 
terfacial micro-cracks. For a more realistic model of the interface, in Figure 1.5(c), we model a period length of the interface as containing a selected number of randomly positioned micro-cracks of randomly generated sizes. The length of a micro-crack is a random variable following a chosen probability density function.

\subsection{Hypersingular Integral Approach for Crack Problems}

In the early 1980s, N. I. Ioakimidis pioneered the formulation of elastic crack problems in terms of integral equations containing Hadamard finite-part integrals (Ioakimidis $[46,47]$ ). To describe such integral equations, he coined the term "hypersingular integral equations". Since then, the hypersingular integral approach for formulating and solving crack problems has attracted the attention of many researchers (see, for example, Ang [7, 8], Ang et al. [11], Ang and Fan [12], Chen [20, 21], García-Sánchez et al. [38], Long and Eshkuvatov [53], Mandal and Chakrabarti [56], Martin [57], Nied [63] and Zhang et al. [84]).

Mathematical approaches for deriving hypersingular integral equations for cracks are given in Ang [9]. For problems with idealized geometries, such as collinear cracks in an infinite elastic space, the hypersingular integral equations may be derived from Fourier integral representations for the elastic displacements and stresses. The derivation of hypersingular integral equations for more general geometries (such as curved cracks and cracks in bodies of finite extent) requires the use of the boundary integral equations in linear elasticity. Boundary integral equations for antiplane and inplane deformations of isotropic and anisotropic elastic bodies are given in Clements [25] and Rizzo [66]. 
The displacement jumps across opposite crack faces appear directly as unknown functions in hypersingular integral equations for crack problems. Once the hypersingular integral equations are solved, the crack-opening displacements are immediately available for further analysis. Other integral approaches for crack problems do not account conveniently for the displacement jumps in such a direct manner. For example, in the distributed dislocation technique for modeling cracks (Hills et al. [44]), the unknowns in the resulting singular integral equations are the dislocational density functions which have to be integrated to recover the crack-opening displacements.

The displacement jumps over the micro-cracks are required in the micromechanical models in Figure 1.5 for estimating the effective stiffness of microscopically damaged interfaces. Because of this, it is advantageous to use the hypersingular integral approach to formulate the micromechanical models. From the analyses in Ang [9] and Ioakimidis [48], the conditions on the interfacial micro-cracks can be formulated in terms of hypersingular boundary integro-differential equations. Another desirable feature of micromechanical models formulated in terms of hypersingular integral or integro-differential equations is that the numerical analysis for estimating the effective stiffness does not require the entire elastic space to be discretized into elements. Generally, the number of unknowns in the numerical analysis is much smaller than that in domain-based numerical methods such as the finite element method. Accurate and efficient numerical techniques for solving hypersingular integral and integro-differential equations may be found in the literature (see, for example, Fannjiang et al. [34], Kaya and Erdogan [51], Linkov [55] and Mandal and Chakrabarti [56]). 


\subsection{The Present Thesis}

\subsubsection{Research Objective and Scope}

The main objective of the present thesis is to develop accurate and efficient micromechanical models based on hypersingular integro-differential equations for analyzing microscopically damaged interfaces between dissimilar orthotropic materials under antiplane and inplane deformations. The models are used to estimate the effective stiffness of microscopically damaged interfaces in multilayered materials. Some specific problems are analyzed in detail to gain useful insights into the modeling and the effective behaviors of micro-cracked interfaces.

\subsubsection{Overview of Remaining Chapters}

The remaining part of the thesis consists of five chapters. The content of each of these chapters is briefly described below.

Chapter 2 gives the mathematical preliminaries needed for the research work in this thesis. They include the governing partial differential equations for orthotropic solids under antiplane and inplane elastostatic deformations, the corresponding boundary integral equations, special Green's functions for perfect interfaces between two elastic half-spaces, definitions of Hadamard finite-part integrals, summation formulae involving digamma and trigamma functions and chi-square distributions.

Chapter 3 presents in detail three different micromechanical models for estimating the effective stiffness of micro-cracked interfaces between orthotropic elastic half-spaces. Cases of antiplane and inplane deformations are considered separately. The first and the second models are highly simplified - they are based on the assumption that the interfacial micro-cracks are identical and 
evenly distributed. In the third model which is based on statistical simulations, the lengths and the positions of the micro-cracks are randomly generated. Suitable Fourier integral representations for the displacements and stresses are employed to formulate and solve the boundary values problems for the models in terms of hypersingular integro-differential equations. The unknown functions in the hypersingular integro-differential equations are the displacement jumps over the damaged parts of the interface. The displacement jumps appear directly in the spring-like model for the interface. Once the hypersingular integro-differential equations are solved, the effective stiffness of the interface can be estimated. Numerical methods for solving the hypersingular integro-differential equations are outlined.

In Chapter 4, micromechanical models like the ones in Chapter 3 are formulated for analyzing a micro-cracked interface between a thin orthotropic layer and an orthotropic half-space. Instead of the Fourier integral approach, the boundary integral equations and the perfect interface Green's functions given in Chapter 2 are employed to derive the hypersingular boundary integrodifferential equations for the models. The unknown functions in the hypersingular boundary integro-differential equations are the displacement jumps across the micro-damaged interface between the thin layer and the half-space and the displacements on the external plane boundary of the layer. Numerical procedures for solving the hypersingular boundary integro-differential equations and estimating the effective stiffness of the interface are outlined here. The effects of the external plane boundary of the thin layer, the micro-crack density and the material properties of the thin layer and the half-space on the effective stiffness of the interface are investigated.

Chapter 5 considers the problem of the interaction of a pair of parallel micro-cracked interfaces in a trimaterial under antiplane and inplane deforma- 
tions. The trimaterial is made of a thin orthotropic elastic layer sandwiched between two orthotropic elastic half-spaces. For the mathematical formulation of the problem, the trimaterial is divided into two subdomains along an artificial plane boundary in the middle of the thin layer. Each of the subdomains may be regarded as a bimaterial consisting of a thin elastic layer and an elastic half-space. Guided by the analysis in Chapter 4, we use the boundary integral equations together with the perfect Green's functions for bimaterials to derive a set of hypersingular boundary integro-differential equations for each of the two subdomains. The unknown functions in the hypersingular integrodifferential equations are the displacement jumps across the micro-cracks on the two interfaces and the displacements and the normal stresses on the artificial plane boundary. Conditions where the displacements and the normal stresses are continuous on the artificial boundary are required to complete the formulation of the problem. Once the hypersingular boundary integrodifferential equations are solved together with the continuity conditions on the artificial boundary, the effective stiffness coefficients of the interfaces may be readily computed. Some specific cases studies are conducted to investigate the effects of the material constants of the thin layer and the two half-spaces, the width of the elastic layer and the densities of the micro-cracks on the effective stiffness coefficients of the two interfaces.

Lastly, Chapter 6 summarizes the main research contributions in this thesis and gives some suggestions for extending the research works here.

\subsection{Publications}

The works reported in the present thesis have been published as follows. 
X. Wang, W. T. Ang and H. Fan, Micro-mechanics models for an imperfect interface under anti-plane shear load: Hypersingular integral formulations, Engineering Analysis with Boundary Elements 36 (2012) 1856-1864.

X. Wang, H. Fan and W. T. Ang, On micromechanical-statistical modeling of microscopically damaged interfaces under antiplane deformations, International Journal of Solids and Structures 51 (2014) 2327-2335.

X. Wang, W. T. Ang and H. Fan, Hypersingular integral and integro-differential micromechanical models for an imperfect interface between a thin orthotropic layer and an orthotropic half-space under inplane elastostatic deformations, Engineering Analysis with Boundary Elements 52 (2015) 32-43.

X. Wang, W. T. Ang and H. Fan, Hypersingular integral equation based micromechanical models for a microscopically damaged antiplane interface between a thin elastic layer and an elastic half space, Applied Mathematical Modelling (accepted for publication). DOI:10.1016/ j.apm.2015.02.002.

X. Wang, W. T. Ang and H. Fan, A micromechanical-statistical model based on hypersingular boundary integral equations for analyzing a pair of parallel interfaces weakened by antiplane micro-cracks, Computers and Structures $\mathbf{1 5 7}$ (2015) 178-188.

X. Wang, W. T. Ang and H. Fan, A micromechanical model based on hypersingular integral and integro-differential equations for a pair of parallel weak interfaces under inplane elastostatic deformations, Computer Modeling in Engineering 83 Sciences (accepted for publication). 


\section{Chapter 2}

\section{Mathematical Preliminaries}

\subsection{Introduction}

The mathematical preliminaries needed in later chapters are given in this chapter. They include the governing partial differential equations for orthotropic solids under antiplane and inplane elastostatic deformations, the corresponding boundary integral equations, special Green's functions for perfect interfaces between two elastic half-spaces, definitions of Hadamard finite-part integrals, summation formulae involving digamma and trigamma functions and chi-square $\left(\chi^{2}\right)$ distributions.

\subsection{Basic Equations of Elasticity}

With reference to a Cartesian coordinate system denoted by $O x_{1} x_{2} x_{3}$, the Cartesian displacements and stresses in a solid are denoted by $u_{k}$ and $\sigma_{i j}$ respectively. For elastostatic problems, $u_{k}$ and $\sigma_{i j}$ are functions of the Cartesian coordinates $x_{1}, x_{2}$ and $x_{3}$ only. In the absence of body force, the stresses are required to satisfy the equilibrium equations (Sadd [70])

$$
\frac{\partial \sigma_{i j}}{\partial x_{j}}=0
$$


Note that lower case Latin subscripts such as $i$ and $j$ have values running from 1 to 3 (in general) and the Einstenian convention of summing over a repeated lowercase Latin subscripts is adopted in this thesis.

According to the generalized Hooke's law, the stresses $\sigma_{i j}$ in an orthotropic elastic solid are related to the displacements $u_{k}$ by

$$
\begin{aligned}
\sigma_{11} & =C_{11} \frac{\partial u_{1}}{\partial x_{1}}+C_{12} \frac{\partial u_{2}}{\partial x_{2}}+C_{13} \frac{\partial u_{3}}{\partial x_{3}}, \\
\sigma_{22} & =C_{12} \frac{\partial u_{1}}{\partial x_{1}}+C_{22} \frac{\partial u_{2}}{\partial x_{2}}+C_{23} \frac{\partial u_{3}}{\partial x_{3}}, \\
\sigma_{12} & =\sigma_{21}=C_{66}\left(\frac{\partial u_{1}}{\partial x_{2}}+\frac{\partial u_{2}}{\partial x_{1}}\right), \\
\sigma_{33} & =C_{13} \frac{\partial u_{1}}{\partial x_{1}}+C_{23} \frac{\partial u_{2}}{\partial x_{2}}+C_{33} \frac{\partial u_{3}}{\partial x_{3}}, \\
\sigma_{13} & =\sigma_{31}=C_{55}\left(\frac{\partial u_{1}}{\partial x_{3}}+\frac{\partial u_{3}}{\partial x_{1}}\right), \\
\sigma_{23} & =\sigma_{32}=C_{44}\left(\frac{\partial u_{2}}{\partial x_{3}}+\frac{\partial u_{3}}{\partial x_{2}}\right),
\end{aligned}
$$

where $C_{i j}$ are the elastic moduli of orthotropic materials satisfying the strict inequality

$$
\begin{aligned}
& C_{11} \xi_{1}^{2}+C_{22} \xi_{2}^{2}+C_{33} \xi_{3}^{2}+2 C_{12} \xi_{1} \xi_{2}+2 C_{13} \xi_{1} \xi_{3}+2 C_{23} \xi_{2} \xi_{3} \\
&+C_{44} \xi_{4}^{2}+C_{55} \xi_{5}^{2}+C_{66} \xi_{6}^{2}>0
\end{aligned}
$$

where $\xi_{k}$ are any real numbers such that $\xi_{1}^{2}+\xi_{2}^{2}+\cdots+\xi_{6}^{2} \neq 0$.

Substitution of (2.2) into (2.1) gives rise to the elliptic system of partial 
differential equations

$$
\begin{aligned}
C_{11} \frac{\partial^{2} u_{1}}{\partial x_{1}^{2}}+\left(C_{12}+C_{66}\right) \frac{\partial^{2} u_{2}}{\partial x_{1} \partial x_{2}}+\left(C_{13}+C_{55}\right) \frac{\partial^{2} u_{3}}{\partial x_{1} \partial x_{3}} & \\
+C_{66} \frac{\partial^{2} u_{1}}{\partial x_{2}^{2}}+C_{55} \frac{\partial^{2} u_{1}}{\partial x_{3}^{2}} & =0, \\
C_{66} \frac{\partial^{2} u_{2}}{\partial x_{1}^{2}}+\left(C_{12}+C_{66}\right) \frac{\partial^{2} u_{1}}{\partial x_{1} \partial x_{2}}+\left(C_{23}+C_{44}\right) \frac{\partial^{2} u_{3}}{\partial x_{2} \partial x_{3}} & =0, \\
+C_{22} \frac{\partial^{2} u_{2}}{\partial x_{2}^{2}}+C_{44} \frac{\partial^{2} u_{2}}{\partial x_{3}^{2}} & =0 \\
C_{55} \frac{\partial^{2} u_{3}}{\partial x_{1}^{2}}+\left(C_{13}+C_{55}\right) \frac{\partial^{2} u_{1}}{\partial x_{1} \partial x_{3}}+\left(C_{23}+C_{44}\right) \frac{\partial^{2} u_{2}}{\partial x_{2} \partial x_{3}} & = \\
+C_{44} \frac{\partial^{2} u_{3}}{\partial x_{2}^{2}}+C_{33} \frac{\partial^{2} u_{3}}{\partial x_{3}^{2}} & =0 .
\end{aligned}
$$

The present thesis considers elastostatic antiplane and inplane deformations of orthotropic bimaterials and trimaterials with geometries independent of the $x_{3}$ coordinate, where the displacements and stresses are functions of only $x_{1}$ and $x_{2}$. The basic equations for antiplane and inplane deformations of orthotropic materials are given below.

\subsubsection{Antiplane Deformations}

For antiplane deformations in which the only non-zero component of the displacements is $u_{3}$, the partial differential equation in (2.4) reduces to

$$
C_{55} \frac{\partial^{2} u_{3}}{\partial x_{1}^{2}}+C_{44} \frac{\partial^{2} u_{3}}{\partial x_{2}^{2}}=0
$$

The partial differential equation in (2.5) has solutions of the general form

$$
u_{3}\left(x_{1}, x_{2}\right)=\operatorname{Re}\left\{f\left(x_{1}+\tau x_{2}\right)\right\}
$$

where Re denotes the real part of a complex number, $f$ is an analytic function of the complex variable $x_{1}+\tau x_{2}$ in the solution domain under consideration 
and $\tau$ is the complex number

$$
\tau=i \sqrt{C_{55} / C_{44}}
$$

where $i=\sqrt{-1}$.

From (2.2), the antiplane shear stresses are

$$
\begin{aligned}
\sigma_{13} & =\sigma_{31}=C_{55} \frac{\partial u_{3}}{\partial x_{1}}, \\
\sigma_{23} & =\sigma_{32}=C_{44} \frac{\partial u_{3}}{\partial x_{2}} .
\end{aligned}
$$

Substitution of (2.6) into (2.8) gives

$$
\begin{aligned}
& \sigma_{31}\left(x_{1}, x_{2}\right)=\sigma_{13}\left(x_{1}, x_{2}\right)=C_{55} \operatorname{Re}\left\{f^{\prime}\left(x_{1}+\tau x_{2}\right)\right\}, \\
& \sigma_{32}\left(x_{1}, x_{2}\right)=\sigma_{23}\left(x_{1}, x_{2}\right)=C_{44} \operatorname{Re}\left\{\tau f^{\prime}\left(x_{1}+\tau x_{2}\right)\right\},
\end{aligned}
$$

where the prime denotes differentiation with respect to the relevant argument.

\subsubsection{Inplane Deformations}

For inplane deformations where $u_{3}=0,(2.4)$ reduces to

$$
\begin{aligned}
& C_{11} \frac{\partial^{2} u_{1}}{\partial x_{1}^{2}}+\left(C_{12}+C_{66}\right) \frac{\partial^{2} u_{2}}{\partial x_{1} \partial x_{2}}+C_{66} \frac{\partial^{2} u_{1}}{\partial x_{2}^{2}}=0, \\
& C_{66} \frac{\partial^{2} u_{2}}{\partial x_{1}^{2}}+\left(C_{12}+C_{66}\right) \frac{\partial^{2} u_{1}}{\partial x_{1} \partial x_{2}}+C_{22} \frac{\partial^{2} u_{2}}{\partial x_{2}^{2}}=0 .
\end{aligned}
$$

The partial differential equations in (2.10) have solutions of the general form

$$
u_{k}\left(x_{1}, x_{2}\right)=\operatorname{Re}\left\{\sum_{\alpha=1}^{2} A_{k \alpha} f_{\alpha}\left(x_{1}+\tau_{\alpha} x_{2}\right)\right\},
$$

where $f_{\alpha}$ are analytic functions of the complex variable $x_{1}+\tau_{\alpha} x_{2}, \tau_{1}$ and $\tau_{2}$ are complex numbers having positive imaginary parts and satisfying the quartic 
equation in $\tau$ given by

$$
\operatorname{det}\left[\begin{array}{cc}
C_{11}+C_{66} \tau^{2} & \left(C_{12}+C_{66}\right) \tau \\
\left(C_{12}+C_{66}\right) \tau & C_{66}+C_{22} \tau^{2}
\end{array}\right]=0
$$

and $A_{k \alpha}$ are constants given by

$$
\left[A_{k \alpha}\right]=\left[\begin{array}{cc}
-\frac{i \tau_{1}\left(C_{12}+C_{66}\right)}{C_{11}+C_{66} \tau_{1}^{2}} & -\frac{i \tau_{2}\left(C_{12}+C_{66}\right)}{C_{11}+C_{66} \tau_{2}^{2}} \\
i & i
\end{array}\right]
$$

Note that lowercase Latin and Greek subscripts henceforth take the values of 1 and 2 .

The inequality in (2.3) ensures that (2.12) has non-real solutions. Further details are given in Clements [25] and Stroh [75]. Later analysis requires inverting the matrix $\left[A_{k \alpha}\right]$. For this purpose, it is assumed here that $\tau_{1} \neq \tau_{2}$.

From (2.2), the inplane stresses are given by

$$
\begin{aligned}
\sigma_{11} & =C_{11} \frac{\partial u_{1}}{\partial x_{1}}+C_{12} \frac{\partial u_{2}}{\partial x_{2}} \\
\sigma_{22} & =C_{12} \frac{\partial u_{1}}{\partial x_{1}}+C_{22} \frac{\partial u_{2}}{\partial x_{2}} \\
\sigma_{12} & =\sigma_{21}=C_{66}\left(\frac{\partial u_{1}}{\partial x_{2}}+\frac{\partial u_{2}}{\partial x_{1}}\right) .
\end{aligned}
$$

For convenience, (2.10) and (2.14) can be respectively written in a more compact form as

$$
c_{i j k p} \frac{\partial^{2} u_{k}}{\partial x_{j} \partial x_{p}}=0
$$

and

$$
\sigma_{i j}=c_{i j k p} \frac{\partial u_{k}}{\partial x_{p}}
$$


where

$$
c_{i j k p}= \begin{cases}C_{11} & \text { if }(i, j, k, p)=(1,1,1,1) \\ C_{12} & \text { if }(i, j, k, p)=(1,1,2,2)=(2,2,1,1), \\ C_{22} & \text { if }(i, j, k, p)=(2,2,2,2) \\ C_{66} & \text { if }(i, j, k, p)=(1,2,1,2)=(2,1,2,1)=(1,2,2,1)=(2,1,1,2), \\ 0 & \text { otherwise. }\end{cases}
$$

Substitution of (2.11) into (2.16) gives rise to the inplane stresses

$$
\sigma_{k j}=\operatorname{Re}\left\{\sum_{\alpha=1}^{2} L_{k j \alpha} f_{\alpha}^{\prime}\left(x_{1}+\tau_{\alpha} x_{2}\right)\right\}
$$

where

$$
L_{i j \alpha}=\left(c_{i j k 1}+\tau_{\alpha} c_{i j k 2}\right) A_{k \alpha}
$$

Note that the summation over repeated lower case Latin subscripts is from 1 to 2 here.

\subsection{Boundary Integral Equations in Elasticity}

Boundary integral equations for antiplane and inplane elastostatic deformations of orthotropic materials are presented here.

\subsubsection{Antiplane Deformations}

If the system (2.5) holds in a two-dimensional region $R$ (bounded by a simple closed curve $C$ ), the antiplane displacement $u_{3}$ satisfies the boundary integral equation (see Clements [25])

$$
\begin{aligned}
u_{3}\left(\xi_{1}, \xi_{2}\right)= & \int_{C}\left[u_{3}\left(x_{1}, x_{2}\right) \Gamma\left(x_{1}, x_{2} ; \xi_{1}, \xi_{2}\right)\right. \\
& \left.-P_{3}\left(x_{1}, x_{2}\right) \Phi\left(x_{1}, x_{2} ; \xi_{1}, \xi_{2}\right)\right] d s\left(x_{1}, x_{2}\right) \\
& \text { for }\left(\xi_{1}, \xi_{2}\right) \text { in the interior of } R,
\end{aligned}
$$


where $P_{3}$ is the $x_{3}$ component of the traction defined by

$$
P_{3}\left(x_{1}, x_{2}\right)=\sigma_{i 3}\left(x_{1}, x_{2}\right) n_{i}\left(x_{1}, x_{2}\right),
$$

$n_{i}$ are the components of the unit outward normal vector to $C$, the functions $\Phi\left(x_{1}, x_{2} ; \xi_{1}, \xi_{2}\right)$ and $\Gamma\left(x_{1}, x_{2} ; \xi_{1}, \xi_{2}\right)$ are defined by

$$
\begin{aligned}
\Phi\left(x_{1}, x_{2} ; \xi_{1}, \xi_{2}\right)= & \frac{1}{2 \pi \sqrt{C_{44} C_{55}}} \operatorname{Re}\left\{\ln \left(x_{1}-\xi_{1}+\tau\left(x_{2}-\xi_{2}\right)\right)\right\} \\
& +\Phi^{*}\left(x_{1}, x_{2} ; \xi_{1}, \xi_{2}\right), \\
\Gamma\left(x_{1}, x_{2} ; \xi_{1}, \xi_{2}\right)= & n_{1} C_{55} \frac{\partial}{\partial x_{1}}\left[\Phi\left(x_{1}, x_{2} ; \xi_{1}, \xi_{2}\right)\right] \\
& +n_{2} C_{44} \frac{\partial}{\partial x_{2}}\left[\Phi\left(x_{1}, x_{2} ; \xi_{1}, \xi_{2}\right)\right],
\end{aligned}
$$

and $\Phi^{*}\left(x_{1}, x_{2} ; \xi_{1}, \xi_{2}\right)$ is any function satisfying

$$
C_{55} \frac{\partial^{2} \Phi^{*}}{\partial x_{1}^{2}}+C_{44} \frac{\partial^{2} \Phi^{*}}{\partial x_{2}^{2}}=0 \text { for }\left(x_{1}, x_{2}\right) \in R .
$$

The boundary integral equation for $\sigma_{3 i}$ may be obtained by substituting (2.20) into (2.8) and interchanging the order of the partial differentiation and integration as given by

$$
\begin{aligned}
\sigma_{3 i}\left(\xi_{1}, \xi_{2}\right)= & \left(\delta_{i 1} \delta_{k 1} C_{55}+\delta_{i 2} \delta_{k 2} C_{44}\right) \int_{C}\left\{u_{3}\left(x_{1}, x_{2}\right) \frac{\partial}{\partial \xi_{k}}\left[\Gamma\left(x_{1}, x_{2} ; \xi_{1}, \xi_{2}\right)\right]\right. \\
& \left.-P_{3}\left(x_{1}, x_{2}\right) \frac{\partial}{\partial \xi_{k}}\left[\Phi\left(x_{1}, x_{2} ; \xi_{1}, \xi_{2}\right)\right]\right\} d s\left(x_{1}, x_{2}\right) \\
& \text { for }\left(\xi_{1}, \xi_{2}\right) \text { in the interior of } R
\end{aligned}
$$

where $\delta_{i p}$ is Kronecker-delta.

The boundary integral equations in (2.24) will be used in later chapters to derive hypersingular integral equations for micromechanical models of weak interfaces under antiplane deformations. 


\subsubsection{Inplane Deformations}

For inplane deformations governed by (2.10), the boundary integral equations are given by (Clements [25] and Clements and Rizzo [26])

$$
\begin{aligned}
u_{k}\left(\xi_{1}, \xi_{2}\right)= & \int_{C}\left[u_{r}\left(x_{1}, x_{2}\right) \Gamma_{r k}\left(x_{1}, x_{2} ; \xi_{1}, \xi_{2}\right)\right. \\
& \left.-P_{r}\left(x_{1}, x_{2}\right) \Phi_{r k}\left(x_{1}, x_{2} ; \xi_{1}, \xi_{2}\right)\right] d s\left(x_{1}, x_{2}\right) \\
& \text { for }\left(\xi_{1}, \xi_{2}\right) \text { in the interior of } R,
\end{aligned}
$$

where $P_{r}\left(x_{1}, x_{2}\right)(r=1$ and 2) are the tractions defined by

$$
P_{r}\left(x_{1}, x_{2}\right)=\sigma_{r j}\left(x_{1}, x_{2}\right) n_{j}\left(x_{1}, x_{2}\right),
$$

the functions $\Phi_{r k}\left(x_{1}, x_{2} ; \xi_{1}, \xi_{2}\right)$ are given by

$$
\begin{aligned}
\Phi_{r k}\left(x_{1}, x_{2} ; \xi_{1}, \xi_{2}\right)= & \frac{1}{2 \pi} \operatorname{Re}\left\{\sum_{\alpha=1}^{2} A_{r \alpha}\left[N_{\alpha p} \ln \left(x_{1}-\xi_{1}+\tau_{\alpha}\left(x_{2}-\xi_{2}\right)\right)\right\} D_{p k}\right. \\
& +\Phi_{r k}^{*}\left(x_{1}, x_{2} ; \xi_{1}, \xi_{2}\right),
\end{aligned}
$$

the matrix $\left[N_{\alpha p}\right]$ is the inverse of $\left[A_{r \alpha}\right]$, the constants $D_{p k}$ are defined by

$$
\operatorname{Im}\left\{\sum_{\alpha=1}^{2} L_{i 2 \alpha} N_{\alpha r}\right\} D_{r p}=\delta_{i p}
$$

$L_{i 2 \alpha}$ is given in (2.19), Im denotes the imaginary part of a complex number, the functions $\Gamma_{r k}\left(x_{1}, x_{2} ; \xi_{1}, \xi_{2}\right)$ are defined in terms of the partial derivatives of $\Phi_{r k}\left(x_{1}, x_{2} ; \xi_{1}, \xi_{2}\right)$ by

$$
\Gamma_{r k}\left(x_{1}, x_{2} ; \xi_{1}, \xi_{2}\right)=n_{j}\left(x_{1}, x_{2}\right) c_{r j p l} \frac{\partial}{\partial x_{l}}\left[\Phi_{p k}\left(x_{1}, x_{2} ; \xi_{1}, \xi_{2}\right)\right],
$$

and $\Phi_{r k}^{*}\left(x_{1}, x_{2} ; \xi_{1}, \xi_{2}\right)$ are any functions satisfying

$$
c_{i j r p} \frac{\partial^{2}}{\partial x_{j} \partial x_{p}}\left[\Phi_{r k}^{*}\left(x_{1}, x_{2} ; \xi_{1}, \xi_{2}\right)\right]=0 .
$$


Substitution of (2.25) into (2.16) gives the boundary integral equations for inplane stresses

$$
\begin{gathered}
\sigma_{i j}\left(\xi_{1}, \xi_{2}\right)=c_{i j k p} \int_{C}\left\{u_{r}\left(x_{1}, x_{2}\right) \frac{\partial}{\partial \xi_{p}}\left[\Gamma_{r k}\left(x_{1}, x_{2} ; \xi_{1}, \xi_{2}\right)\right]\right. \\
\left.-P_{r}\left(x_{1}, x_{2}\right) \frac{\partial}{\partial \xi_{p}}\left[\Phi_{r k}\left(x_{1}, x_{2} ; \xi_{1}, \xi_{2}\right)\right]\right\} d s\left(x_{1}, x_{2}\right) \\
\text { for }\left(\xi_{1}, \xi_{2}\right) \text { in the interior of } R .
\end{gathered}
$$

The boundary integral equations in (2.30) will be used in later chapters to derive hypersingular integral equations for micromechanical models of weak interfaces under inplane deformations.

\subsection{Special Green's Functions for Perfect In- terfaces}

Consider a bimaterial consisting of two dissimilar orthotropic materials occupying the half-spaces $x_{2}>0$ and $x_{2}<0$. The two half-spaces are perfectly bonded on the plane $x_{2}=0$. Refer to Figure 2.1. Green's functions satisfying the conditions on the perfectly bonded interface are given below.

\subsubsection{Antiplane Green's Function}

For the antiplane Green's function, the function $\Phi^{*}$ in $(2.22)$ is chosen in such a way that the function $\Phi$ satisfies the interfacial conditions

$$
\left.\begin{array}{c}
\Phi\left(x_{1}, 0^{+} ; \xi_{1}, \xi_{2}\right)=\Phi\left(x_{1}, 0^{-} ; \xi_{1}, \xi_{2}\right) \\
\left.C_{44}^{(1)} \frac{\partial \Phi}{\partial x_{2}}\right|_{x_{2}=0^{+}}=\left.C_{44}^{(2)} \frac{\partial \Phi}{\partial x_{2}}\right|_{x_{2}=0^{-}}
\end{array}\right\} \text {for }-\infty<x_{1}<\infty .
$$

where the superscripts (1) and (2) are used to denote the elastic moduli of materials 1 and 2 respectively. 


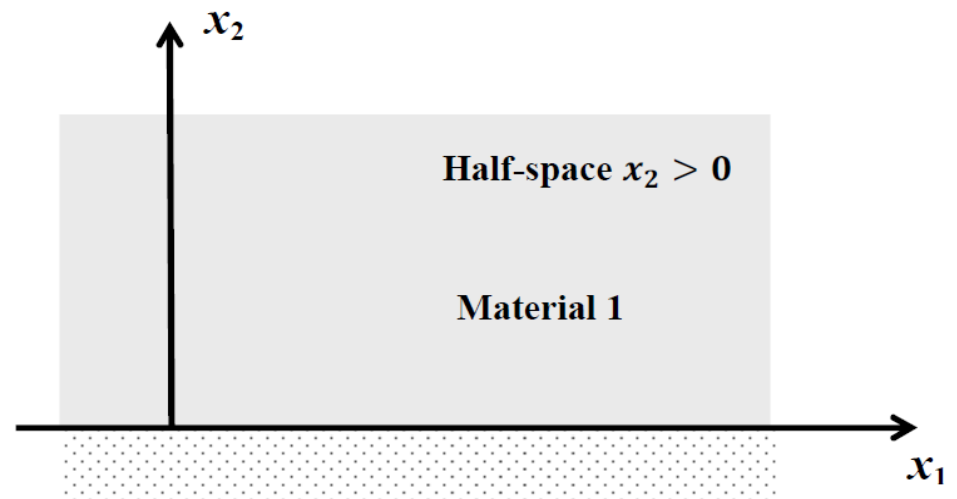

Materal 2

Half $\mathrm{spce} x_{2}<0$

Figure 2.1: A sketch of two dissimilar orthotropic half-spaces perfectly bonded along $x_{2}=0$.

The Green's function is given in complete form in Berger and Karageorghis [14] as

$$
\begin{aligned}
& \Phi\left(x_{1}, x_{2} ; \xi_{1}, \xi_{2}\right) \\
= & H\left(x_{2}\right) H\left(\xi_{2}\right)\left[\frac{1}{2 \pi \beta^{(1)}} \operatorname{Re}\left\{\ln \left(x_{1}-\xi_{1}+\tau^{(1)}\left(x_{2}-\xi_{2}\right)\right)\right\}\right. \\
& \left.\frac{\beta^{(1)}-\beta^{(2)}}{2 \pi \beta^{(1)}\left(\beta^{(1)}+\beta^{(2)}\right)} \operatorname{Re}\left\{\ln \left(x_{1}+\tau^{(1)} x_{2}-\xi_{1}-\bar{\tau}^{(1)} \xi_{2}\right)\right\}\right] \\
& +H\left(-x_{2}\right) H\left(-\xi_{2}\right)\left[\frac{1}{2 \pi \beta^{(2)}} \operatorname{Re}\left\{\ln \left(x_{1}-\xi_{1}+\tau^{(2)}\left(x_{2}-\xi_{2}\right)\right)\right\}\right. \\
& \left.\frac{\beta^{(2)}-\beta^{(1)}}{2 \pi \beta^{(2)}\left(\beta^{(1)}+\beta^{(2)}\right)} \operatorname{Re}\left\{\ln \left(x_{1}+\tau^{(2)} x_{2}-\xi_{1}-\bar{\tau}^{(2)} \xi_{2}\right)\right\}\right] \\
& +H\left(x_{2}\right) H\left(-\xi_{2}\right)\left[\frac{1}{\pi\left(\beta^{(1)}+\beta^{(2)}\right)} \operatorname{Re}\left\{\ln \left(x_{1}+\tau^{(1)} x_{2}-\xi_{1}-\tau^{(2)} \xi_{2}\right)\right\}\right] \\
& +H\left(-x_{2}\right) H\left(\xi_{2}\right)\left[\frac{1}{\pi\left(\beta^{(1)}+\beta^{(2)}\right)} \operatorname{Re}\left\{\ln \left(x_{1}+\tau^{(2)} x_{2}-\xi_{1}-\tau^{(1)} \xi_{2}\right)\right\}\right],
\end{aligned}
$$


where the overhead bar denotes the complex conjugate of a complex number, $\beta^{(p)}=\sqrt{C_{44}^{(p)} C_{55}^{(p)}}, \tau^{(p)}=i \sqrt{C_{55}^{(p)} / C_{44}^{(p)}}$ and $H(x)$ is the unit-step Heaviside function defined by

$$
H(x)= \begin{cases}1 & \text { if } x>0 \\ 0 & \text { if } x<0\end{cases}
$$

The function $\Gamma\left(x_{1}, x_{2} ; \xi_{1}, \xi_{2}\right)$ corresponding to $\Phi\left(x_{1}, x_{2} ; \xi_{1}, \xi_{2}\right)$ in (2.32) is given by

$$
\begin{aligned}
& \Gamma\left(x_{1}, x_{2} ; \xi_{1}, \xi_{2}\right) \\
= & H\left(x_{2}\right) H\left(\xi_{2}\right)\left[\frac{1}{2 \pi \beta^{(1)}} \operatorname{Re}\left\{\frac{L_{i}^{(1)} n_{i}\left(x_{1}, x_{2}\right)}{x_{1}-\xi_{1}+\tau^{(1)}\left(x_{2}-\xi_{2}\right)}\right\}\right. \\
& \left.\frac{\beta^{(1)}-\beta^{(2)}}{2 \pi \beta^{(1)}\left(\beta^{(1)}+\beta^{(2)}\right)} \operatorname{Re}\left\{\frac{L_{i}^{(1)} n_{i}\left(x_{1}, x_{2}\right)}{x_{1}+\tau^{(1)} x_{2}-\xi_{1}-\bar{\tau}^{(1)} \xi_{2}}\right\}\right] \\
& +H\left(-x_{2}\right) H\left(-\xi_{2}\right)\left[\frac{1}{2 \pi \beta^{(2)}} \operatorname{Re}\left\{\frac{L_{i}^{(2)} n_{i}\left(x_{1}, x_{2}\right)}{x_{1}-\xi_{1}+\tau^{(2)}\left(x_{2}-\xi_{2}\right)}\right\}\right. \\
& \left.\frac{\beta^{(2)}-\beta^{(1)}}{2 \pi \beta^{(2)}\left(\beta^{(1)}+\beta^{(2)}\right)} \operatorname{Re}\left\{\frac{L_{i}^{(2)} n_{i}\left(x_{1}, x_{2}\right)}{x_{1}+\tau^{(2)} x_{2}-\xi_{1}-\bar{\tau}^{(2)} \xi_{2}}\right\}\right] \\
& +H\left(x_{2}\right) H\left(-\xi_{2}\right)\left[\frac{1}{\pi\left(\beta^{(1)}+\beta^{(2)}\right)} \operatorname{Re}\left\{\frac{L_{i}^{(1)} n_{i}\left(x_{1}, x_{2}\right)}{x_{1}+\tau^{(1)} x_{2}-\xi_{1}-\tau^{(2)} \xi_{2}}\right\}\right] \\
& +H\left(-x_{2}\right) H\left(\xi_{2}\right)\left[\frac{1}{\pi\left(\beta^{(1)}+\beta^{(2)}\right)} \operatorname{Re}\left\{\frac{L_{i}^{(2)} n_{i}\left(x_{1}, x_{2}\right)}{x_{1}+\tau^{(2)} x_{2}-\xi_{1}-\tau^{(1)} \xi_{2}}\right\}\right],
\end{aligned}
$$

where $L_{i}^{(p)}$ is defined by

$$
L_{i}^{(p)}=\delta_{i 1} C_{55}^{(p)}+\delta_{i 2} \tau^{(p)} C_{44}^{(p)}
$$

\subsubsection{Inplane Green's Function}

For the inplane Green's function, the functions $\Phi_{r k}^{*}$ in (2.27) are chosen such that

$$
\left.\begin{array}{rl}
\Phi_{r k}\left(x_{1}, 0^{+} ; \xi_{1}, \xi_{2}\right) & =\Phi_{r k}\left(x_{1}, 0^{-} ; \xi_{1}, \xi_{2}\right) \\
\left.c_{i 2 r l}^{(1)} \frac{\partial \Phi_{r k}}{\partial x_{l}}\right|_{x_{2}=0^{+}} & =\left.c_{i 2 r l}^{(2)} \frac{\partial \Phi_{r k}}{\partial x_{l}}\right|_{x_{2}=0^{-}}
\end{array}\right\} \text {for }-\infty<x_{1}<\infty .
$$


The Green's function is originally given in Berger and Tewary [16]. It can also be found in Chen and Ang [22] where it is written as

$$
\begin{aligned}
& \Phi_{r k}\left(x_{1}, x_{2} ; \xi_{1}, \xi_{2}\right) \\
= & \frac{1}{2 \pi} \operatorname{Re}\left\{H ( x _ { 2 } ) H ( \xi _ { 2 } ) \sum _ { \alpha = 1 } ^ { 2 } A _ { r \alpha } ^ { ( 1 ) } \left[N_{\alpha p}^{(1)} \ln \left(x_{1}-\xi_{1}+\tau_{\alpha}^{(1)}\left(x_{2}-\xi_{2}\right)\right)\right.\right. \\
& \left.+\sum_{\beta=1}^{2} Q_{\alpha \beta p}^{(1)} \ln \left(x_{1}-\xi_{1}+\tau_{\alpha}^{(1)} x_{2}-\bar{\tau}_{\beta}^{(1)} \xi_{2}\right)\right] D_{p k}^{(1)} \\
& +H\left(-x_{2}\right) H\left(\xi_{2}\right) \sum_{\alpha=1}^{2} A_{r \alpha}^{(2)} \sum_{\beta=1}^{2} Q_{\alpha \beta p}^{(2)} \ln \left(x_{1}-\xi_{1}+\tau_{\alpha}^{(2)} x_{2}-\tau_{\beta}^{(1)} \xi_{2}\right) D_{p k}^{(1)} \\
& +H\left(x_{2}\right) H\left(-\xi_{2}\right) \sum_{\alpha=1}^{2} A_{r \alpha}^{(1)} \sum_{\beta=1}^{2} T_{\alpha \beta p}^{(1)} \ln \left(x_{1}-\xi_{1}+\tau_{\alpha}^{(1)} x_{2}-\tau_{\beta}^{(2)} \xi_{2}\right) D_{p k}^{(2)} \\
& +H\left(-x_{2}\right) H\left(-\xi_{2}\right) \sum_{\alpha=1}^{2} A_{r \alpha}^{(2)}\left[N_{\alpha p}^{(2)} \ln \left(x_{1}-\xi_{1}+\tau_{\alpha}^{(2)}\left(x_{2}-\xi_{2}\right)\right)\right. \\
& \left.\left.+\sum_{\beta=1}^{2} T_{\alpha \beta p}^{(2)} \ln \left(x_{1}-\xi_{1}+\tau_{\alpha}^{(2)} x_{2}-\bar{\tau}_{\beta}^{(2)} \xi_{2}\right)\right] D_{p k}^{(2)}\right\}
\end{aligned}
$$

where the constants $Q_{\alpha \beta p}^{(p)}$ and $T_{\alpha \beta p}^{(p)}$ are defined by

$$
\begin{aligned}
N_{\gamma k}^{(2)}\left(A_{k \beta}^{(1)} N_{\beta p}^{(1)}+\sum_{\alpha=1}^{2} \bar{A}_{k \alpha}^{(1)} \bar{Q}_{\alpha \beta p}^{(1)}\right) & =Q_{\gamma \beta p}^{(2)}, \\
M_{\gamma k}^{(2)}\left(L_{k 2 \beta}^{(1)} N_{\beta p}^{(1)}+\sum_{\alpha=1}^{2} \bar{L}_{k 2 \alpha}^{(1)} \bar{Q}_{\alpha \beta p}^{(1)}\right) & =Q_{\gamma \beta p}^{(2)}, \\
N_{\gamma k}^{(1)}\left(A_{k \beta}^{(2)} N_{\beta p}^{(2)}+\sum_{\alpha=1}^{2} \bar{A}_{k \alpha}^{(2)} \bar{T}_{\alpha \beta p}^{(2)}\right) & =T_{\gamma \beta p}^{(1)}, \\
M_{\gamma k}^{(1)}\left(L_{k 2 \beta}^{(2)} N_{\beta p}^{(2)}+\sum_{\alpha=1}^{2} \bar{L}_{k 2 \alpha}^{(2)} \bar{T}_{\alpha \beta p}^{(2)}\right) & =T_{\gamma \beta p}^{(1)},
\end{aligned}
$$

the constants $\tau_{\alpha}^{(p)}, A_{r \alpha}^{(p)}, L_{r j \alpha}^{(p)}, N_{\alpha p}^{(p)}$ and $D_{r k}^{(p)}$ are respectively $\tau_{\alpha}, A_{r \alpha}, N_{\alpha p}, L_{r j \alpha}$ and $D_{r k}$ in Subsection 2.3.2 computed by using $c_{i j k \ell}=c_{i j k \ell}^{(p)}$ and $\left[M_{r k}^{(p)}\right]$ is the inverse of $\left[L_{r 2 k}^{(p)}\right]$. 
The functions $\Gamma_{r k}\left(x_{1}, x_{2} ; \xi_{1}, \xi_{2}\right)$ corresponding to $\Phi_{r k}\left(x_{1}, x_{2} ; \xi_{1}, \xi_{2}\right)$ in $(2.37)$ are given by

$$
\begin{aligned}
& \Gamma_{r k}\left(x_{1}, x_{2} ; \xi_{1}, \xi_{2}\right) \\
= & \frac{1}{2 \pi} \operatorname{Re}\left\{H ( x _ { 2 } ) H ( \xi _ { 2 } ) \sum _ { \alpha = 1 } ^ { 2 } L _ { r j \alpha } ^ { ( 1 ) } n _ { j } ( x _ { 1 } , x _ { 2 } ) \left[\frac{N_{\alpha p}^{(1)}}{x_{1}-\xi_{1}+\tau_{\alpha}^{(1)}\left(x_{2}-\xi_{2}\right)}\right.\right. \\
& \left.+\sum_{\beta=1}^{2} \frac{Q_{\alpha \beta p}^{(1)}}{x_{1}-\xi_{1}+\tau_{\alpha}^{(1)} x_{2}-\bar{\tau}_{\beta}^{(1)} \xi_{2}}\right] D_{p k}^{(1)} \\
& +H\left(-x_{2}\right) H\left(\xi_{2}\right) \sum_{\alpha=1}^{2} L_{r j \alpha}^{(2)} n_{j}\left(x_{1}, x_{2}\right) \sum_{\beta=1}^{2} \frac{Q_{\alpha \beta p}^{(2)}}{x_{1}-\xi_{1}+\tau_{\alpha}^{(2)} x_{2}-\tau_{\beta}^{(1)} \xi_{2}} D_{p k}^{(1)} \\
& +H\left(x_{2}\right) H\left(-\xi_{2}\right) \sum_{\alpha=1}^{2} L_{r j \alpha}^{(1)} n_{j}\left(x_{1}, x_{2}\right) \sum_{\beta=1}^{2} \frac{T_{\alpha \beta p}^{(1)}}{\left(x_{1}-\xi_{1}+\tau_{\alpha}^{(1)} x_{2}-\tau_{\beta}^{(2)} \xi_{2}\right)} D_{p k}^{(2)} \\
& +H\left(-x_{2}\right) H\left(-\xi_{2}\right) \sum_{\alpha=1}^{2} L_{r j \alpha}^{(2)} n_{j}\left(x_{1}, x_{2}\right)\left[\frac{N_{\alpha p}^{(2)}}{x_{1}-\xi_{1}+\tau_{\alpha}^{(2)}\left(x_{2}-\xi_{2}\right)}\right. \\
& \left.\left.+\sum_{\beta=1}^{2} \frac{T_{\alpha \beta p}^{(2)}}{x_{1}-\xi_{1}+\tau_{\alpha}^{(2)} x_{2}-\bar{\tau}_{\beta}^{(2)} \xi_{2}}\right] D_{p k}^{(2)}\right\} .
\end{aligned}
$$

\subsection{Hadamard Finite-part Integrals}

If the point $\left(\xi_{1}, \xi_{2}\right)$ in $(2.24)$ and $(2.30)$ approaches a point $\left(y_{1}, y_{2}\right)$ on a smooth part of the boundary $C$, the boundary integrals become improper and must be interpreted as Cauchy principal and Hadamard finite-part integrals. Basic definitions of these integrals are given below.

Cauchy principal and Hadamard finite-part integrals are respectively defined by(see Ang [9], Kaya and Erdogan [51], Mandal and Chakrabarti [56] and Martin [57])

$$
f_{a}^{b} \frac{F(t) d t}{t-x}=\lim _{\epsilon \rightarrow 0^{+}}\left\{\int_{a}^{x-\epsilon} \frac{F(t) d t}{t-x}+\int_{x+\epsilon}^{b} \frac{F(t) d t}{t-x}\right\} \text { for } a<x<b,
$$


and

$$
f_{a}^{b} \frac{F(t)}{(x-t)^{2}} d t=\lim _{\epsilon \rightarrow 0^{+}}\left\{\int_{a}^{x-\epsilon} \frac{F(t)}{(x-t)^{2}} d t+\int_{\substack{x+\epsilon \\ \text { for } a<x<b,}}^{b} \frac{F(t)}{(x-t)^{2}} d t-\frac{2 F(x)}{\epsilon}\right\}
$$

where $F(t)$ is a well defined function for $a<t<b$. Note the use of the symbols - and $=$ superimposed on the integral sign is to denote that the integrals are to be interpreted in the Cauchy principal and Hadamard finite-part sense.

As shown in Ang [9], the two integrals are related to each other by

$$
f_{a}^{b} \frac{F(t) d t}{(t-x)^{2}}=\frac{d}{d x}\left[f_{a}^{b} \frac{F(t) d t}{t-x}\right] \text { for } a<x<b
$$

An alternative but equivalent definition for the Hadamard finite-part integrals is given by (see Ang and Clements [10])

$$
\begin{aligned}
& f_{a}^{b} \frac{F(t)}{(x-t)^{2}} d t= \lim _{\epsilon \rightarrow 0^{+}} \int_{a}^{b} \frac{\left((t-x)^{2}-\epsilon^{2}\right) F(t)}{\left[(x-t)^{2}+\epsilon^{2}\right]^{2}} d t \\
&= \lim _{\epsilon \rightarrow 0^{+}}\left\{\frac{(t-x)^{2} F(t)}{\left[(x-t)^{2}+\epsilon^{2}\right]^{2}} d t-\frac{\pi F(x)}{2 \epsilon}\right\} \\
&-\frac{F(x)}{2}\left[\frac{1}{b-x}-\frac{1}{a-x}\right] \\
& \quad \text { for } a<x<b .
\end{aligned}
$$

\subsection{Summation Formulae Involving Digamma and Trigamma Functions}

In later chapters, the following summation formulae are used to sum up the contributions of periodically distributed micro-cracks and other periodic bound- 
ary (if any) to the stress field in a multilayered material:

$$
\left.\begin{array}{r}
\sum_{n=1}^{\infty}\left(\frac{1}{z_{1}-n z_{2}}+\frac{1}{z_{1}+n z_{2}}\right)=\frac{1}{z_{2}}\left[\Psi_{0}\left(1-\frac{z_{1}}{z_{2}}\right)-\Psi_{0}\left(1+\frac{z_{1}}{z_{2}}\right)\right] \\
\sum_{n=1}^{\infty} \frac{1}{\left(z_{1} \pm n z_{2}\right)^{2}}=\frac{1}{z_{2}^{2}} \Psi_{1}\left(1 \pm \frac{z_{1}}{z_{2}}\right) \\
\text { for }\left|z_{2}\right| \neq 0 \text { and } \operatorname{Re}\left\{1 \pm \frac{z_{1}}{z_{2}}\right\}>0
\end{array}\right\}
$$

where $z_{1}$ and $z_{2}$ are complex constants, $\Psi_{0}(z)$ is the digamma function and $\Psi_{1}(z)$ is the trigamma function.

In Abramowitz and Stegun [5], the special functions $\Psi_{0}(z)$ and $\Psi_{1}(z)$ are defined by:

$$
\left.\begin{array}{c}
\Psi_{0}(z)=\int_{0}^{\infty}\left(\frac{e^{-t}}{t}-\frac{e^{-z t}}{1-e^{-t}}\right) d t \\
\Psi_{1}(z)=\int_{0}^{\infty} \frac{t e^{-z t}}{1-e^{-t}} d t
\end{array}\right\} \text { for } \operatorname{Re}\{z\}>0 .
$$

Mathematical algorithms for calculating $\Psi_{0}(z)$ and $\Psi_{1}(z)$ are available in the literature (see, for example, Bernardo [17]). A strategy for a very accurate and efficient computation is to compute $\Psi_{0}(z)$ and $\Psi_{1}(z)$ very accurately for a sufficiently large value of $|z|$ by using the asymptotic formulae (for large $|z|$ )

$$
\begin{aligned}
& \Psi_{0}(z)=\ln (z)-\frac{1}{2 z}-\frac{1}{12 z^{2}}+\frac{1}{120 z^{4}}-\frac{1}{252 z^{6}}+O\left(\frac{1}{z^{8}}\right), \\
& \Psi_{1}(z)=\frac{1}{z}+\frac{1}{2 z^{2}}+\frac{1}{6 z^{3}}-\frac{1}{30 z^{5}}+\frac{1}{42 z^{7}}+O\left(\frac{1}{z^{9}}\right)
\end{aligned}
$$

and obtain the values of $\Psi_{0}(z)$ and $\Psi_{1}(z)$ for smaller values of $|z|$ by the recursive relations

$$
\begin{aligned}
& \Psi_{0}(z)=-\frac{1}{z}+\Psi_{0}(1+z), \\
& \Psi_{1}(z)=\frac{1}{z^{2}}+\Psi_{1}(1+z) .
\end{aligned}
$$




\subsection{Chi-square $\left(\chi^{2}\right)$ Distribution}

In this thesis, one of the micromechanical models for microscopically damaged interfaces requires the interfacial micro-cracks to be randomly generated. We use the chi-square $\left(\chi^{2}\right)$ distribution to generate randomly the lengths of the micro-cracks.

(a) $\chi^{2}(5)$

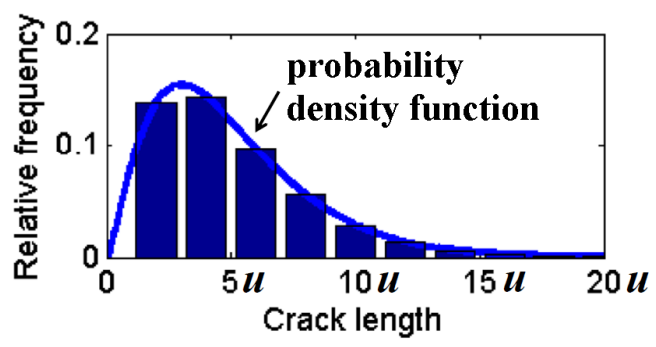

(b) $\chi^{2}(10)$

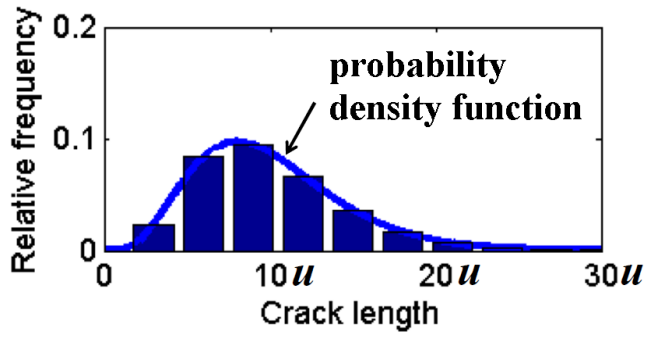

(c) $\chi^{2}(25)$

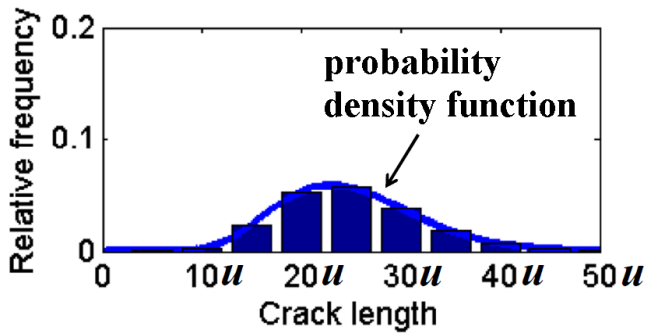

Figure 2.2: Relative frequency distribution of the micro-crack lengths generated by using the $\chi^{2}$ distribution of degrees of freedom 5, 10 and 25. A unit length of the micro-crack is denoted by $u$. 
The $\chi^{2}$ distribution of degree of freedom $m$ (denoted by $\chi^{2}(m)$ ) has the probability density function given by (Abramowitz and Stegun [5])

$$
f_{m}(x)=\left\{\begin{array}{cc}
\frac{x^{-1+m / 2} e^{-x / 2}}{2^{m / 2} \Gamma(m / 2)} & \text { for } x \geq 0, \\
0 & \text { for } x<0
\end{array}\right.
$$

where $m$ is a positive integer and $\Gamma$ is the gamma function.

Figure 2.2 shows the relative frequency distribution of the micro-crack lengths randomly generated using $\chi^{2}(5), \chi^{2}(10)$ and $\chi^{2}(25)$. If the $\chi^{2}$ distribution has a lower degree of freedom, the micro-crack length distribution is skewed towards shorter length, that is, there is a larger number of shorter micro-cracks. See Figure 2.2(a). If the $\chi^{2}$ distribution has a relatively large degree of freedom (for example, $\chi^{2}(25)$ in Figure 2.2(c)), the micro-crack length distribution becomes less skewed and more normal like. For more realistic simulations, a lower degree of freedom should be used in the $\chi^{2}$ distribution. 



\section{Chapter 3}

\section{Weak Interfaces between Two Orthotropic Half-spaces}

\subsection{Introduction}

Micromechanical models for analyzing a microscopically damaged interface between two orthotropic elastic half-spaces under antiplane and inplane deformations are proposed in this chapter. The interface is modeled as containing micro-cracks as explained in Section 1.2. The three micromechanical models as sketched in Figure 1.5 are formulated in terms of boundary value problems governed by the equilibrium equations of elasticity.

Suitable Fourier integral representations for the displacement and the stress fields are used to solve the boundary value problems in terms of hypersingular integro-differential equations. The unknown functions in the hypersingular integro-differential equations are the displacement jumps over the damaged parts of the interface. The displacement jumps appear directly in the spring-like model for the interface. Once the hypersingular integro-differential equations are solved, the effective stiffness of the interface can be estimated. Numerical methods are used for solving the hypersingular integro-differential equations. 


\subsection{A Micro-cracked Interface}

With reference to a Cartesian coordinate system $\mathrm{Ox}_{1} x_{2} x_{3}$, consider two dissimilar homogeneous orthotropic elastic half-spaces occupying the regions $x_{2}>0$ and $x_{2}<0$. The plane interface $x_{2}=0$ on which the elastic half-spaces are joined is microscopically damaged. The bimaterial undergoes either an antiplane or inplane elastostatic deformation which depends only on $x_{1}$ and $x_{2}$. The basic equations for the antiplane or inplane deformation are given in Section 2.2 with the elastic constants for the orthotropic materials given by

$$
\begin{aligned}
& \left(C_{11}, C_{22}, C_{12}, C_{66}, C_{44}, C_{55}\right) \\
= & \begin{cases}\left(C_{11}^{(1)}, C_{22}^{(1)}, C_{12}^{(1)}, C_{66}^{(1)}, C_{44}^{(1)}, C_{55}^{(1)}\right) & \text { for } x_{2}>0, \\
\left(C_{11}^{(2)}, C_{22}^{(2)}, C_{12}^{(2)}, C_{66}^{(2)}, C_{44}^{(2)}, C_{55}^{(2)}\right) & \text { for } x_{2}<0 .\end{cases}
\end{aligned}
$$

The microscopically damaged interface is modeled as containing periodic arrays of interfacial micro-cracks. More specifically, $M$ arbitrarily located micro-cracks of possibly different lengths lie on the part of the interface where $0<x_{1}<L, x_{2}=0$. The tips of a typical $m$-th micro-crack on this part of the interface are given by $\left(a^{(m)}, 0\right)$ and $\left(b^{(m)}, 0\right)$, where $a^{(m)}$ and $b^{(m)}$ are constants such that $0<a^{(1)}<b^{(1)}<a^{(2)}<b^{(2)}<\cdots<a^{(M)}<b^{(M)}<L$. On the remaining parts of the interface, the micro-cracks are given by $a^{(m)}+n L<x_{1}<$ $b^{(m)}+n L$ for $m=1,2, \cdots, M$ and $n= \pm 1, \pm 2, \cdots$, that is, the remaining micro-cracks are periodically distributed exact replicas of the $M$ micro-cracks on the interval $0<x_{1}<L, x_{2}=0$. Refer to Figure 3.1.

The damage ratio $\rho$ which gives the micro-crack density of the interface is defined as follows:

$$
\rho=\frac{1}{L} \sum_{m=1}^{M}\left(b^{(m)}-a^{(m)}\right) .
$$

At the macro level, the micro-cracked interface can be modeled as a springlike interface with interfacial conditions given by (1.1). In Cartesian coordi- 


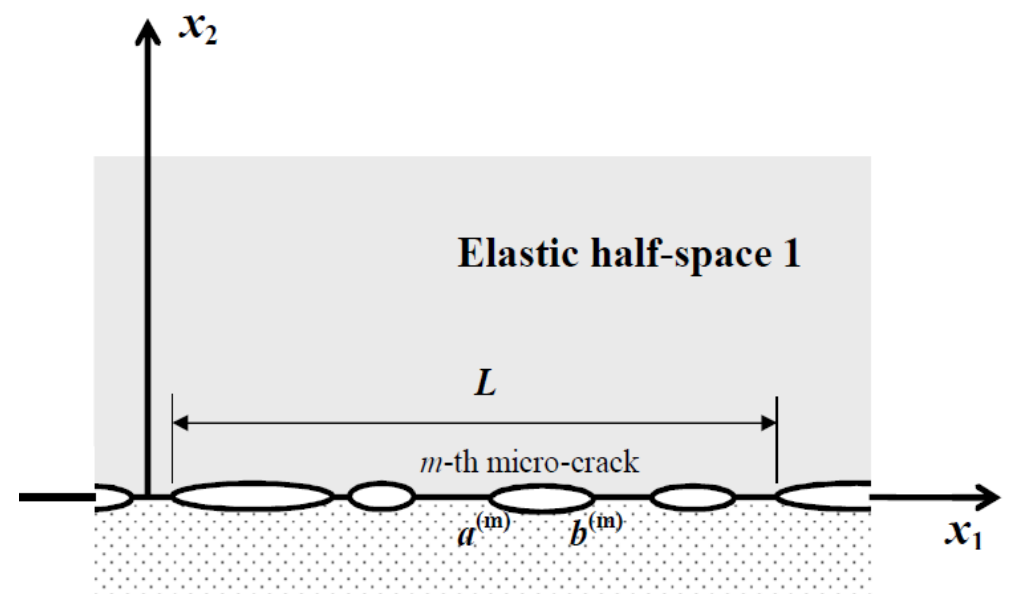

\section{Elastic half space 2}

Figure 3.1: A sketch of a period length of the micro-cracked interface between two dissimilar half-spaces.

nates, the spring-like interfacial conditions for the plane interface $x_{2}=0$ can be written as

$$
\left[\begin{array}{ll}
k_{11} & k_{12} \\
k_{21} & k_{22}
\end{array}\right]\left[\begin{array}{l}
\Delta u_{1}\left(x_{1}\right) \\
\Delta u_{2}\left(x_{1}\right)
\end{array}\right]=\left[\begin{array}{l}
\sigma_{12}\left(x_{1}, 0^{+}\right) \\
\sigma_{22}\left(x_{1}, 0^{+}\right)
\end{array}\right]=\left[\begin{array}{l}
\sigma_{12}\left(x_{1}, 0^{-}\right) \\
\sigma_{22}\left(x_{1}, 0^{-}\right)
\end{array}\right]
$$

for inplane deformations, (3.3)

and

$$
k_{33} \Delta u_{3}\left(x_{1}\right)=\sigma_{32}\left(x_{1}, 0^{+}\right)=\sigma_{32}\left(x_{1}, 0^{-}\right) \text {for antiplane deformations, }
$$

where $\Delta u_{k}\left(x_{1}\right)=u_{k}\left(x_{1}, 0^{+}\right)-u_{k}\left(x_{1}, 0^{-}\right), \Delta u_{3}\left(x_{1}\right)=u_{3}\left(x_{1}, 0^{+}\right)-u_{3}\left(x_{1}, 0^{-}\right)$, $u_{k}$ and $\sigma_{k j}(k, j=1,2)$ are respectively the Cartesian inplane displacements and stresses, $u_{3}$ and $\sigma_{3 j}(j=1,2)$ are respectively the antiplane displacement 
and stresses and $k_{i j}(i, j=1,2)$ and $k_{33}$ are the effective stiffness coefficients of the micro-cracked interface.

For the macro-level spring model defined by (3.3) (for inplane deformations) to be valid, the external tensile load acting on the bimaterial along the $x_{2}$ direction is assumed to be sufficiently large (dominant) so that the macrolevel interfacial displacement $\Delta u_{2}$ is positive.

The problem of interest here is to estimate the effective stiffness coefficients $k_{i j}(i, j=1,2)$ and $k_{33}$ for the micro-cracked interface between the orthotropic half-spaces. To estimate the effective stiffness coefficients, three specific micromechanical models are proposed here for the micro-cracked interface. Before discussing the models in detail, we provide in Section 3.3 below a derivation of hypersingular integro-differential equations for the conditions on the micro-cracks on the interface between the two orthotropic half-spaces. These hypersingular integro-differential equations or their modified forms are used in the mathematical formulations of the three micromechanical models.

\subsection{Hypersingular Integro-differential Equations for Interfacial Micro-cracks}

The interfacial micro-cracks in the bimaterial in Section 3.2 are assumed to become traction free under the action of external loads at infinity. Following closely the analysis in Ang [9], we express the displacements and stresses in terms of suitable Fourier integral representations to derive the hypersingular integro-differential equations for traction-free conditions on the interfacial micro-cracks. 


\subsubsection{Antiplane Deformations}

If the antiplane displacement and stresses are written as

$$
\begin{aligned}
u_{3} & =u_{3}^{(\mathrm{ext})}+u_{3}^{(\mathrm{imp})}, \\
\sigma_{3 r} & =\sigma_{3 r}^{(\mathrm{ext})}+\sigma_{3 r}^{(\mathrm{imp})},
\end{aligned}
$$

then the conditions on the interface may be rewritten as

$$
\begin{aligned}
& \sigma_{32}^{(\mathrm{imp})}\left(x_{1}, 0^{ \pm}\right)=-\sigma_{32}^{(\mathrm{ext})}\left(x_{1}, 0^{ \pm}\right) \text {for }\left(x_{1}, 0\right) \in D, \\
& \left.\begin{array}{rl}
\Delta u_{3}^{(\mathrm{imp})}\left(x_{1}\right) & =0 \\
\sigma_{32}^{(\mathrm{imp})}\left(x_{1}, 0^{+}\right) & =\sigma_{32}^{(\mathrm{imp})}\left(x_{1}, 0^{-}\right)
\end{array}\right\} \text {for }\left(x_{1}, 0\right) \in P,
\end{aligned}
$$

where $u_{3}^{(\mathrm{ext})}$ and $\sigma_{32}^{(\mathrm{ext})}$ are respectively the displacement and the stress fields in the bimaterial for the corresponding case where the interface does not contain any micro-crack, $\Delta u_{3}^{(\mathrm{imp})}\left(x_{1}\right)=u_{3}^{(\mathrm{imp})}\left(x_{1}, 0^{+}\right)-u_{3}^{(\mathrm{imp})}\left(x_{1}, 0^{-}\right)$, and $u_{3}^{(\mathrm{imp})}$ and $\sigma_{32}^{(\mathrm{imp})}$ are respectively the displacement and the stress induced by the microcracks and $D$ and $P$ denote the micro-cracked parts and the perfectly bonded parts of the interface respectively.

The loads $\sigma_{32}^{(\text {ext })}\left(x_{1}, 0\right)$ on the micro-cracks are assumed to be periodic functions of $x_{1}$ with period $L$ so that $u_{3}^{(\mathrm{imp})}$ and $\sigma_{32}^{(\mathrm{imp})}$ are also periodic functions of $x_{1}$ with period $L$. With $u_{3}^{\text {(ext) }}$ and $\sigma_{32}^{\text {(ext) }}$ assumed known, the boundary value problem for determining $u_{3}^{(\mathrm{imp})}$ and $\sigma_{32}^{(\mathrm{imp})}$ is to solve (2.5) together with (3.1) subject to the interfacial conditions in (3.6) and the far field condition given by $\sigma_{3 k}^{(\mathrm{imp})} \rightarrow 0$ as $\left|x_{2}\right| \rightarrow \infty$.

From (2.6) and (2.9), we write the antiplane fields $u_{3}^{(\mathrm{imp})}$ and $\sigma_{32}^{(\mathrm{imp})}$ in (3.5) respectively as

$$
u_{3}^{(\mathrm{imp})}\left(\xi_{1}, \xi_{2}\right)= \begin{cases}\operatorname{Re}\left\{f\left(\xi_{1}+\tau^{(1)} \xi_{2}\right)\right\} & \text { for } \xi_{2}>0 \\ \operatorname{Re}\left\{g\left(\xi_{1}+\tau^{(2)} \xi_{2}\right)\right\} & \text { for } \xi_{2}<0\end{cases}
$$

and

$$
\sigma_{32}^{(\mathrm{imp})}\left(\xi_{1}, \xi_{2}\right)= \begin{cases}\operatorname{Re}\left\{i \beta^{(1)} f^{\prime}\left(\xi_{1}+\tau^{(1)} \xi_{2}\right)\right\} & \text { for } \xi_{2}>0, \\ \operatorname{Re}\left\{i \beta^{(2)} g^{\prime}\left(\xi_{1}+\tau^{(2)} \xi_{2}\right)\right\} & \text { for } \xi_{2}<0\end{cases}
$$


where $\beta^{(1)}$ and $\beta^{(2)}$ are real numbers defined below (2.32) and calculated using the elastic coefficients of half-spaces 1 and 2 respectively, and $f$ and $g$ are analytic functions expressed in the Fourier integral form

$$
\begin{aligned}
& f\left(\xi_{1}+\tau^{(1)} \xi_{2}\right)=\int_{0}^{\infty} E^{(1)}(\zeta) \exp \left(i \zeta\left[\xi_{1}+\tau^{(1)} \xi_{2}\right]\right) d \zeta \\
& g\left(\xi_{1}+\tau^{(2)} \xi_{2}\right)=\int_{0}^{\infty} E^{(2)}(\zeta) \exp \left(-i \zeta\left[\xi_{1}+\tau^{(2)} \xi_{2}\right]\right) d \zeta
\end{aligned}
$$

and $E^{(1)}(\zeta)$ and $E^{(2)}(\zeta)$ are complex functions yet to be determined.

From (3.6), it is required that $\sigma_{32}^{(\mathrm{imp})}\left(\xi_{1}, 0^{+}\right)=\sigma_{32}^{(\mathrm{imp})}\left(\xi_{1}, 0^{-}\right)$for $-\infty<\xi_{1}<$ $-\infty$. This interfacial condition is satisfied if $E^{(1)}(\zeta)$ and $E^{(2)}(\zeta)$ are given by

$$
\beta^{(1)} E^{(1)}(\zeta)=-\beta^{(2)} \bar{E}^{(2)}(\zeta)=\phi(\zeta),
$$

where $\phi(\zeta)$ is an arbitrary function yet to be determined.

If $\phi(\zeta)$ is given by

$$
\phi(\zeta)=i T \sum_{n=-\infty}^{\infty} \sum_{m=1}^{M} \int_{a^{(m)}+n L}^{b^{(m)}+n L} r\left(x_{1}\right) \exp \left(-i \zeta x_{1}\right) d x_{1},
$$

where $T$ is a real number and $r\left(x_{1}\right)$ is a real function, then

$$
\begin{aligned}
& u_{3}^{(\mathrm{imp})}\left(\xi_{1}, 0^{+}\right)-u_{3}^{(\mathrm{imp})}\left(\xi_{1}, 0^{-}\right) \\
= & -T \lim _{\varepsilon \rightarrow 0^{+}} \sum_{n=-\infty}^{\infty} \sum_{m=1}^{M} \int_{a^{(m)}+n L}^{b^{(m)}+n L} r\left(x_{1}\right) \operatorname{Re}\left\{\frac{1}{i \beta^{(1)}\left(\xi_{1}-x_{1}+\tau^{(1)} \varepsilon\right)}\right. \\
& \left.+\frac{1}{i \beta^{(2)}\left(\xi_{1}-x_{1}-\bar{\tau}^{(2)} \varepsilon\right)}\right\} d x_{1} .
\end{aligned}
$$

For $\xi_{1} \notin\left[a^{(m)}+n L, b^{(m)}+n L\right]$, the integrands of the integrals on the right hand side of (3.12) are not singular for $\varepsilon=0$. Hence the integrals are proper for $\varepsilon=0$. The order of the limit and the integration can be interchanged. 
Thus,

$$
\begin{aligned}
& \lim _{\varepsilon \rightarrow 0^{+}} \int_{a^{(m)}+n L}^{b^{(m)}+n L} r\left(x_{1}\right) \operatorname{Re}\left\{\frac{1}{i \beta^{(1)}\left(\xi_{1}-x_{1}+\tau^{(1)} \varepsilon\right)}\right. \\
& \left.+\frac{1}{i \beta^{(2)}\left(\xi_{1}-x_{1}-\bar{\tau}^{(2)} \varepsilon\right)}\right\} d x_{1} \\
= & \int_{a^{(m)}+n L}^{b^{(m)}+n L} r\left(x_{1}\right) \operatorname{Re}\left\{\frac{1}{i \beta^{(1)}\left(\xi_{1}-x_{1}\right)}+\frac{1}{i \beta^{(2)}\left(\xi_{1}-x_{1}\right)}\right\} d x_{1} \\
= & 0 \text { for } \xi_{1} \notin\left[a^{(m)}+n L, b^{(m)}+n L\right],
\end{aligned}
$$

that is, the interfacial condition $u_{3}^{(\mathrm{imp})}\left(\xi_{1}, 0^{+}\right)=u_{3}^{(\mathrm{imp})}\left(\xi_{1}, 0^{-}\right)$in (3.6) is satisfied on the perfectly bonded areas of the interface.

If the constant $T$ is chosen such that

$$
\pi T\left(\frac{1}{i \beta^{(1)}}+\frac{1}{i \beta^{(2)}}\right)=-i
$$

then the real function $r\left(x_{1}\right)$ can be shown to be equal to $\Delta u_{3}^{(\mathrm{imp})}\left(x_{1}\right)$ which is the displacement jump across the opposite crack faces (Ang [9]).

Substitution of (3.9) into (3.8) together with (3.10) and (3.11) gives rise to

$$
\begin{aligned}
\sigma_{32}^{(\mathrm{imp})}\left(\xi_{1}, 0^{+}\right)= & T \operatorname{Re}\left\{\sum_{m=1}^{M} \lim _{\varepsilon \rightarrow 0^{+}} \int_{a^{(m)}}^{b^{(m)}} \frac{\Delta u_{3}^{(\mathrm{imp})}\left(x_{1}\right)}{\left(\xi_{1}-x_{1}+\tau^{(1)} \varepsilon\right)^{2}} d x_{1}\right\} \\
& +T \operatorname{Re}\left\{\sum_{n=1}^{\infty} \sum_{m=1}^{M} \lim _{\varepsilon \rightarrow 0^{+}} \int_{a^{(m)}+n L}^{b^{(m)}+n L} \frac{\Delta u_{3}^{(\mathrm{imp})}\left(x_{1}\right)}{\left(\xi_{1}-x_{1}+\tau^{(1)} \varepsilon\right)^{2}} d x_{1}\right\} \\
& +T \operatorname{Re}\left\{\sum_{n=1}^{\infty} \sum_{m=1}^{M} \lim _{\varepsilon \rightarrow 0^{+}} \int_{a^{(m)}-n L}^{b^{(m)}-n L} \frac{\Delta u_{3}^{(\mathrm{imp})}\left(x_{1}\right)}{\left(\xi_{1}-x_{1}+\tau^{(1)} \varepsilon\right)^{2}} d x_{1}\right\} .
\end{aligned}
$$


The first term on the right hand side of (3.14) can be written as

$$
\begin{aligned}
& T \operatorname{Re}\left\{\sum_{m=1}^{M} \lim _{\varepsilon \rightarrow 0^{+}} \int_{a^{(m)}}^{b^{(m)}} \frac{\Delta u_{3}^{(\mathrm{imp})}\left(x_{1}\right)}{\left(\xi_{1}-x_{1}+\tau^{(1)} \varepsilon\right)^{2}} d x_{1}\right\} \\
= & T \operatorname{Re}\left\{\lim _{\varepsilon \rightarrow 0^{+}} \int_{a^{(k)}}^{b^{(k)}} \frac{\Delta u_{3}^{(\mathrm{imp})}\left(x_{1}\right)}{\left(\xi_{1}-x_{1}+\tau^{(1)} \varepsilon\right)^{2}} d x_{1}\right\}+T \sum_{\substack{m=1 \\
m \neq k}}^{M} \int_{a^{(m)}}^{b^{(m)}} \frac{\Delta u_{3}^{(\mathrm{imp})}\left(x_{1}\right)}{\left(\xi_{1}-x_{1}\right)^{2}} d x_{1} \\
& \text { for } a^{(k)}<\xi_{1}<b^{(k)}(k=1,2, \ldots, M) .
\end{aligned}
$$

Note that the integrals over $a^{(m)}<x_{1}<b^{(m)}$ (on the left hand side of (3.15)) are proper for $\varepsilon=0$ if $m \neq k$. Hence the order of the limit and the integration can be directly interchanged if $m \neq k$ to give the second term on the right hand side of (3.15).

On the left hand side of (3.15), the integrand of the integral over $a^{(k)}<$ $x_{1}<b^{(k)}$ is singular for $a^{(k)}<\xi_{1}<b^{(k)}$. As shown in Ang [9], the first term on the right hand side of (3.15) can be interpreted in terms of the Hadamard finite-part integral defined in (2.42). More specifically, (3.15) can be rewritten as

$$
\begin{aligned}
& T \operatorname{Re}\left\{\sum_{m=1}^{M} \lim _{\varepsilon \rightarrow 0^{+}} \int_{a^{(m)}}^{b^{(m)}} \frac{\Delta u_{3}^{(\mathrm{imp})}\left(x_{1}\right)}{\left(\xi_{1}-x_{1}+\tau^{(1)} \varepsilon\right)^{2}} d x_{1}\right\} \\
= & T f_{a^{(k)}}^{b^{(k)}} \frac{\Delta u_{3}^{(\mathrm{imp})}\left(x_{1}\right)}{\left(x_{1}-\xi_{1}\right)^{2}} d x_{1}+T \sum_{\substack{m=1 \\
m \neq k}}^{M} \int_{a^{(m)}}^{b^{(m)}} \frac{\Delta u_{3}^{(\mathrm{imp})}\left(x_{1}\right)}{\left(x_{1}-\xi_{1}\right)^{2}} d x_{1} \\
& \text { for } a^{(k)}<\xi_{1}<b^{(k)}(k=1,2, \ldots, M) .
\end{aligned}
$$

From the summation formula in (2.45), if $a^{(k)}<\xi_{1}<b^{(k)}$ then the second and third terms on the right hand side of (3.14) can be rewritten respectively 
as

$$
\begin{aligned}
& T \sum_{n=1}^{\infty} \sum_{m=1}^{M} \lim _{\varepsilon \rightarrow 0^{+}} \int_{a^{(m)}+n L}^{b^{(m)}+n L} \frac{\Delta u_{3}^{(\mathrm{imp})}\left(x_{1}\right)}{\left(\xi_{1}-x_{1}+\tau^{(1)} \varepsilon\right)^{2}} d x_{1} \\
= & T \sum_{m=1}^{M} \sum_{n=1}^{\infty} \int_{a^{(m)}+n L}^{b^{(m)}+n L} \frac{\Delta u_{3}^{(\mathrm{imp})}\left(x_{1}\right)}{\left(x_{1}-\xi_{1}\right)^{2}} d x_{1} \\
= & \frac{T}{L^{2}} \sum_{m=1}^{M} \int_{a^{(m)}}^{b^{(m)}} \Delta u_{3}^{(\mathrm{imp})}\left(x_{1}\right) \Psi_{1}\left(\frac{L+x_{1}-\xi_{1}}{L}\right) d x_{1},
\end{aligned}
$$

and

$$
\begin{aligned}
& T \sum_{n=1}^{\infty} \sum_{m=1}^{M} \lim _{\varepsilon \rightarrow 0^{+}} \int_{a^{(m)}-n L}^{b^{(m)}-n L} \frac{\Delta u_{3}^{(\mathrm{imp})}\left(x_{1}\right)}{\left(\xi_{1}-x_{1}+\tau^{(1)} \varepsilon\right)^{2}} d x_{1} \\
= & T \sum_{m=1}^{M} \sum_{n=1}^{\infty} \int_{a^{(m)}-n L}^{b^{(m)}-n L} \frac{\Delta u_{3}^{(\mathrm{imp})}\left(x_{1}\right)}{\left(x_{1}-\xi_{1}\right)^{2}} d x_{1} \\
= & \frac{T}{L^{2}} \sum_{m=1}^{M} \int_{a^{(m)}}^{b^{(m)}} \Delta u_{3}^{(\mathrm{imp})}\left(x_{1}\right) \Psi_{1}\left(\frac{L+\xi_{1}-x_{1}}{L}\right) d x_{1},
\end{aligned}
$$

where $\Psi_{1}$ is the trigamma function defined in (2.45).

From (3.14)-(3.18), the conditions on the micro-cracks as given in (3.6) are given by the hypersingular integral equations

$$
\begin{aligned}
& f_{a^{(k)}}^{b^{(k)}} \frac{\Delta u_{3}^{(\mathrm{imp})}\left(x_{1}\right)}{\left(x_{1}-\xi_{1}\right)^{2}} d x_{1}+\sum_{\substack{m=1 \\
m \neq k}}^{M} \int_{a^{(m)}}^{b^{(m)}} \frac{\Delta u_{3}^{(\mathrm{imp})}\left(x_{1}\right)}{\left(x_{1}-\xi_{1}\right)^{2}} d x_{1} \\
& +\sum_{m=1}^{M} \int_{a^{(m)}}^{b^{(m)}} \Delta u_{3}^{(\mathrm{imp})}\left(x_{1}\right) \Theta\left(x_{1}, \xi_{1}\right) d x_{1} \\
& =-\frac{1}{T} \sigma_{32}^{(\mathrm{ext})}\left(\xi_{1}, 0^{+}\right) \text {for } a^{(k)}<\xi_{1}<b^{(k)}(k=1,2, \ldots, M),
\end{aligned}
$$

where

$$
\Theta\left(x_{1}, \xi_{1}\right)=\frac{1}{L^{2}} \Psi_{1}\left(\frac{L+x_{1}-\xi_{1}}{L}\right)+\frac{1}{L^{2}} \Psi_{1}\left(\frac{L+\xi_{1}-x_{1}}{L}\right) .
$$




\subsubsection{Inplane Deformations}

For inplane deformations, the displacements $u_{r}$ and the stresses $\sigma_{r j}$ are written as

$$
\begin{aligned}
u_{r} & =u_{r}^{(\mathrm{ext})}+u_{r}^{(\mathrm{imp})}, \\
\sigma_{r j} & =\sigma_{r j}^{(\mathrm{ext})}+\sigma_{r j}^{(\mathrm{imp})},
\end{aligned}
$$

then the conditions on the interface may be rewritten as

$$
\begin{aligned}
& \sigma_{r 2}^{(\mathrm{imp})}\left(x_{1}, 0^{ \pm}\right)=-\sigma_{r 2}^{(\mathrm{ext})}\left(x_{1}, 0^{ \pm}\right) \text {for }\left(x_{1}, 0\right) \in D, \\
& \left.\begin{array}{rl}
\Delta u_{r}^{(\mathrm{imp})}\left(x_{1}\right) & =0 \\
\sigma_{r 2}^{(\mathrm{imp})}\left(x_{1}, 0^{+}\right) & =\sigma_{r 2}^{(\mathrm{imp})}\left(x_{1}, 0^{-}\right)
\end{array}\right\} \text {for }\left(x_{1}, 0\right) \in P,
\end{aligned}
$$

where $u_{r}^{\text {(ext) }}$ and $\sigma_{r j}^{\text {(ext) }}$ are respectively the displacement and the stress fields in the bimaterial for the corresponding case where the interface does not contain any micro-crack, $\Delta u_{r}^{(\mathrm{imp})}\left(x_{1}\right)=u_{r}^{(\mathrm{imp})}\left(x_{1}, 0^{+}\right)-u_{r}^{(\mathrm{imp})}\left(x_{1}, 0^{-}\right), u_{r}^{(\mathrm{imp})}$ and $\sigma_{r 2}^{(\mathrm{imp})}$ are respectively the displacements and the stresses induced by the micro-cracks and $D$ and $P$ respectively denote all the micro-cracked parts and the perfectly bonded parts on the whole interface.

As in the case for antiplane deformations, the loads $\sigma_{r 2}^{(\text {ext })}\left(x_{1}, 0\right)$ on the micro-cracks are assumed to be periodic functions of $x_{1}$ with period $L$. With $u_{r}^{(\text {ext })}$ and $\sigma_{r j}^{(\text {ext })}$ assumed known, the boundary value problem for determining $u_{r}^{(\mathrm{imp})}$ and $\sigma_{r j}^{(\mathrm{imp})}$ is to solve (2.10) together with (3.1) subject to the interfacial conditions in (3.22) and the far field condition given by $\sigma_{r j}^{(\mathrm{imp})} \rightarrow 0$ as $\left|x_{2}\right| \rightarrow \infty$.

The elastic fields $u_{r}^{(\mathrm{imp})}$ and $\sigma_{r 2}^{(\mathrm{imp})}$ in (2.11) and (2.18) are respectively given by

$$
u_{r}^{(\mathrm{imp})}\left(\xi_{1}, \xi_{2}\right)=\left\{\begin{array}{cc}
\operatorname{Re}\left\{\sum_{\alpha=1}^{2} A_{r \alpha}^{(1)} f_{\alpha}\left(\xi_{1}+\tau_{\alpha}^{(1)} \xi_{2}\right)\right\} & \text { for } \xi_{2}>0, \\
\operatorname{Re}\left\{\sum_{\alpha=1}^{2} A_{r \alpha}^{(2)} g_{\alpha}\left(\xi_{1}+\tau_{\alpha}^{(2)} \xi_{2}\right)\right\} & \text { for } \xi_{2}<0,
\end{array}\right.
$$


and

$$
\sigma_{r j}^{(\mathrm{imp})}\left(\xi_{1}, \xi_{2}\right)=\left\{\begin{array}{cc}
\operatorname{Re}\left\{\sum_{\alpha=1}^{2} L_{r j \alpha}^{(1)} f_{\alpha}^{\prime}\left(\xi_{1}+\tau_{\alpha}^{(1)} \xi_{2}\right)\right\} & \text { for } \xi_{2}>0, \\
\left.\operatorname{Re}\left\{\sum_{\alpha=1}^{2} L_{r j \alpha}^{(2)} g_{\alpha}^{\prime}\left(\xi_{1}+\tau_{\alpha}^{(2)} \xi_{2}\right)\right]\right\} & \text { for } \xi_{2}<0,
\end{array}\right.
$$

where $\tau_{\alpha}^{(p)}, A_{r \alpha}^{(p)}$ and $L_{r j \alpha}^{(p)}$ are respectively the constants $\tau_{\alpha}, A_{r \alpha}$ and $L_{r j \alpha}$ in (2.11) and (2.18) calculated using the elastic moduli of half-space $p(p=1,2)$ and the complex functions $f_{\alpha}$ and $g_{\alpha}$ are taken to be in the Fourier integral form:

$$
\begin{aligned}
f_{\alpha}\left(\xi_{1}+\tau_{\alpha}^{(2)} \xi_{2}\right) & \left.=\int_{0}^{\infty} E_{\alpha}^{(1)}(\zeta) \exp \left(i \zeta\left[\xi_{1}+\tau_{\alpha}^{(1)} \xi_{2}\right)\right]\right) d \zeta \\
g_{\alpha}\left(\xi_{1}+\tau_{\alpha}^{(2)} \xi_{2}\right) & =\int_{0}^{\infty} E_{\alpha}^{(2)}(\zeta) \exp \left(-i \zeta\left[\xi_{1}+\tau_{\alpha}^{(2)} \xi_{2}\right]\right) d \zeta
\end{aligned}
$$

and $E_{\alpha}^{(1)}(\zeta)$ and $E_{\alpha}^{(2)}(\zeta)$ are complex functions yet to be determined.

To ensure that the stresses $\sigma_{r 2}^{(\mathrm{imp})}$ are continuous on the entire interface $x_{2}=0$, we take

$$
\begin{aligned}
& E_{\alpha}^{(1)}(\zeta)=M_{\alpha q}^{(1)} \phi_{q}(\zeta) \\
& E_{\alpha}^{(2)}(\zeta)=M_{\alpha q}^{(2)} \bar{\phi}_{q}(\zeta)
\end{aligned}
$$

where $M_{\alpha q}^{(p)}$ are defined below by (2.39) and $\phi_{q}(\zeta)$ are functions yet to be determined.

If $\phi_{q}(\zeta)$ is chosen to be

$$
\phi_{q}(\zeta)=i Q_{q j} \sum_{n=-\infty}^{\infty} \sum_{m=1}^{M} \int_{a^{(m)}+n L}^{b^{(m)}+n L} r_{j}\left(x_{1}\right) \exp \left(-i \zeta x_{1}\right) d x_{1}
$$


where $Q_{q j}$ are complex constants and $r_{j}\left(x_{1}\right)$ are real functions, then

$$
\begin{aligned}
& u_{r}^{(\mathrm{imp})}\left(\xi_{1}, 0^{+}\right)-u_{r}^{(\mathrm{imp})}\left(\xi_{1}, 0^{-}\right) \\
= & -\lim _{\varepsilon \rightarrow 0^{+}} \sum_{n=-\infty}^{\infty} \sum_{m=1}^{M} \int_{a^{(m)}+n L}^{b^{(m)}+n L} r_{j}\left(x_{1}\right) \operatorname{Re}\left\{\sum _ { \alpha = 1 } ^ { 2 } Q _ { q j } \left[\frac{A_{r \alpha}^{(1)} M_{\alpha q}^{(1)}}{\xi_{1}-x_{1}+\tau_{\alpha}^{(1)} \varepsilon}\right.\right. \\
& \left.\left.-\frac{\bar{A}_{r \alpha}^{(2)} \bar{M}_{\alpha q}^{(2)}}{\xi_{1}-x_{1}-\bar{\tau}_{\alpha}^{(2)} \varepsilon}\right]\right\} d x_{1} .
\end{aligned}
$$

Proceeding in a similar way as in Subsection 3.3.1 for antiplane deformations, we may show that (3.28) satisfies $u_{r}^{(\mathrm{imp})}\left(\xi_{1}, 0^{+}\right)-u_{r}^{(\mathrm{imp})}\left(\xi_{1}, 0^{-}\right)=0$ on the perfectly bonded parts of the interface and $r_{j}\left(x_{1}\right)=\Delta u_{j}^{(\mathrm{imp})}\left(x_{1}\right)$ is satisfied on the micro-cracks if the constants $Q_{q j}$ are implicitly given by

$$
\pi Q_{j r} \sum_{\alpha=1}^{2}\left[A_{k \alpha}^{(1)} M_{\alpha j}^{(1)}-\bar{A}_{k \alpha}^{(2)} \bar{M}_{\alpha j}^{(2)}\right]=-i \delta_{k r} .
$$

Also, from (3.23)-(3.27) and (2.45), the conditions in (3.22) for the interfacial micro-cracks can be expressed in terms of the hypersingular integrodifferential equations

$$
\begin{aligned}
& f_{a^{(k)}}^{b^{(k)}} \frac{\operatorname{Re}\left\{Q_{j r}\right\} \Delta u_{r}^{(\mathrm{imp})}\left(x_{1}\right)}{\left(x_{1}-\xi_{1}\right)^{2}} d x_{1}+\sum_{\substack{m=1 \\
m \neq k}}^{M} \int_{a^{(m)}}^{b^{(m)}} \frac{\operatorname{Re}\left\{Q_{j r}\right\} \Delta u_{r}^{(\mathrm{imp})}\left(x_{1}\right)}{\left(x_{1}-\xi_{1}\right)^{2}} d x_{1} \\
& +\sum_{m=1}^{M} \int_{a^{(m)}}^{b^{(m)}} \operatorname{Re}\left\{Q_{j r}\right\} \Delta u_{r}^{(\mathrm{imp})}\left(x_{1}\right) \Theta\left(x_{1}, \xi_{1}\right) d x_{1}-\operatorname{Im}\left\{\pi Q_{j r}\right\} \frac{d \Delta u_{r}^{(\mathrm{imp})}\left(\xi_{1}\right)}{d \xi_{1}} \\
& =-\sigma_{j 2}^{(\mathrm{ext})}\left(\xi_{1}, 0^{+}\right) \text {for } a^{(k)}<\xi_{1}<b^{(k)}(k=1,2, \ldots, M) .
\end{aligned}
$$

\subsection{Micromechanical Models for Estimating Ef- fective Stiffness}

The three micromechanical models for estimating the effective stiffness of the micro-cracked interface between the two orthotropic elastic half-spaces are presented below. The effective stiffness is independent of the prescribed loads 
acting on the interface. In the models, the loads are taken to be

$$
\begin{aligned}
& \sigma_{32}^{\text {(ext) }}\left(x_{1}, 0^{ \pm}\right)=\sigma_{3} \text { for antiplane deformations, } \\
& \sigma_{r 2}^{\text {(ext) }}\left(x_{1}, 0^{ \pm}\right)=\sigma_{r} \text { for inplane deformations }
\end{aligned}
$$

where $\sigma_{r}(r=1,2)$ and $\sigma_{3}$ are positive constants. For inplane deformations, we assume that $\sigma_{2}>>\sigma_{1}$, so that the micro-cracks mainly open up under the action of the loads.

\subsubsection{Three-phase Model}

In the three-phase model here, a period interval of the micro-cracked interface in Section 3.2 is modeled as comprising three parts: a representative (typical) micro-crack, a perfectly bonded part and an effective region. The geometry of the interface is periodic with period $L$. The effective regions are essentially spring-like interfaces with a yet to be determined effective stiffness. A geometrical sketch of the three-phase model for the micro-cracked interface is shown in Figure 3.2. On the period interval of the interface $x_{2}=0$ shown in Figure 3.2 , the interval $c^{(1)}<x_{1}<d^{(1)}$ is occupied by the micro-crack, the intervals $0<x_{1}<c^{(1)}$ and $d^{(1)}<x_{1}<c^{(2)}$ are perfectly bonded, and the interval $c^{(2)}<x_{1}<d^{(2)}$ is the effective region. The micro-crack is centrally located in the interval $0<x_{1}<c^{(2)}$. The length of the effective region is assumed to be very large compared to the lengths of the micro-crack and the perfectly bonded parts, that is, we assume $d^{(2)}-c^{(2)}>>c^{(2)}$. Note that $c^{(1)}=c^{(2)}-d^{(1)}$ and $d^{(2)}=L$.

For the three-phase model, the damage ratio for the interface corresponding to $(3.2)$ is given by

$$
\rho=\frac{d^{(1)}-c^{(1)}}{c^{(2)}}
$$




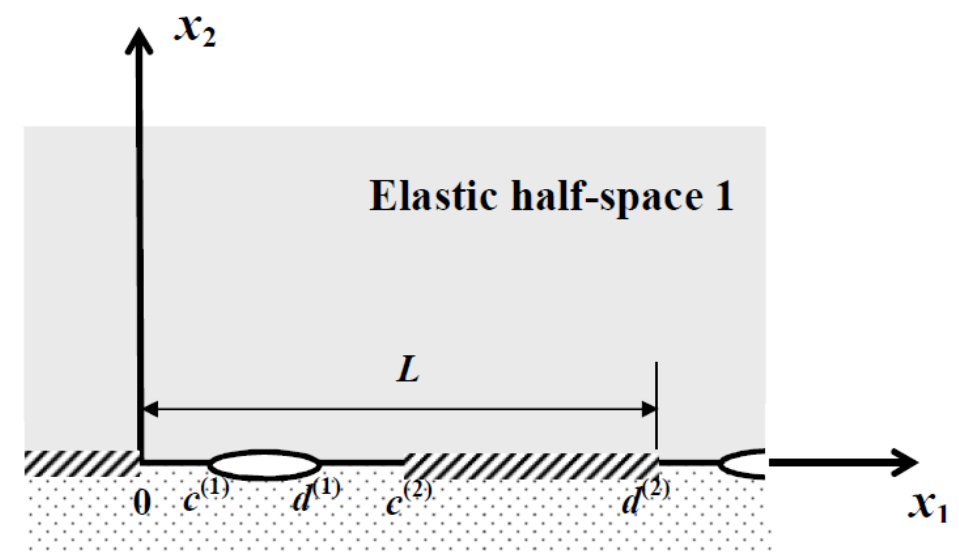

\section{Elastic half-space 2}

Figure 3.2: A geometrical sketch of the three-phase model for the micro-cracked interface.

For antiplane deformations, the interfacial conditions for the three-phase model are given by

$$
\begin{aligned}
& \sigma_{32}^{(\mathrm{imp})}\left(x_{1}, 0^{ \pm}\right)=-\sigma_{3} \text { for }\left(x_{1}, 0\right) \in D, \\
& \left.\begin{array}{rl}
\Delta u_{3}^{(\mathrm{imp})}\left(x_{1}\right) & =0 \\
\sigma_{32}^{(\mathrm{imp})}\left(x_{1}, 0^{+}\right) & =\sigma_{32}^{(\mathrm{imp})}\left(x_{1}, 0^{-}\right)
\end{array}\right\} \text {for }\left(x_{1}, 0\right) \in P, \\
& k_{33} \Delta u_{3}^{(\mathrm{imp})}\left(x_{1}\right)=\sigma_{3}+\sigma_{32}^{(\mathrm{imp})}\left(x_{1}, 0^{ \pm}\right) \text {for }\left(x_{1}, 0\right) \in E,
\end{aligned}
$$

where $D$ and $P$ are defined below (3.22), and $E$ denotes all the effective regions on the whole interface.

For inplane deformations, the interfacial conditions are given by

$$
\begin{aligned}
& \sigma_{r 2}^{(\mathrm{imp})}\left(x_{1}, 0^{ \pm}\right)=-\sigma_{r} \text { for }\left(x_{1}, 0\right) \in D, \\
& \left.\begin{array}{rl}
\Delta u_{r}^{(\mathrm{imp})}\left(x_{1}\right) & =0 \\
\sigma_{r 2}^{(\mathrm{imp})}\left(x_{1}, 0^{+}\right) & =\sigma_{r 2}^{(\mathrm{imp})}\left(x_{1}, 0^{-}\right)
\end{array}\right\} \text {for }\left(x_{1}, 0\right) \in P, \\
& k_{i j} \Delta u_{j}^{(\mathrm{imp})}\left(x_{1}\right)=\sigma_{i}+\sigma_{i 2}^{(\mathrm{imp})}\left(x_{1}, 0^{ \pm}\right) \text {for }\left(x_{1}, 0\right) \in E \text {. }
\end{aligned}
$$


The effective region $E$ behaves like the spring-like interface but with unknown (yet to determined) effective stiffness coefficients $k_{i j}$ (for inplane deformations) and $k_{33}$ (for antiplane deformations). To account for the unknown stiffness coefficients, additional equations are needed to complete the formulation of the model.

For antiplane deformations, to account for $k_{33}$, the additional equation is given by

$$
\frac{k_{33} \int_{c^{(1)}}^{d^{(1)}} \Delta u_{3}^{(\mathrm{imp})}\left(x_{1}\right) d x_{1}}{c^{(2)}}=\sigma_{3} .
$$

For inplane deformations, the additional equations accounting for $k_{i j}$ are given by

$$
\frac{k_{i j} \int_{c^{(1)}}^{d^{(1)}} \Delta u_{j}^{(\mathrm{imp})}\left(x_{1}\right) d x_{1}}{c^{(2)}}=\sigma_{i} .
$$

The relations (3.35) and (3.36) involve averaging the displacement jumps and the tractions over the part of the interface defined by $0<x_{1}<c^{(2)}, x_{2}=0$. Note that the displacement jumps are zero over the perfectly bonded parts of the interface.

The analysis in Section 3.3 can be easily modified to derive hypersingular integro-differential equations for the conditions over a period interval of the interface in the three-phase model.

For antiplane deformations, the hypersingular integral equations are

$$
\begin{aligned}
& f_{c^{(q)}}^{d^{(q)}} \frac{\Delta u_{3}^{(\mathrm{imp})}\left(x_{1}\right)}{\left(x_{1}-\xi_{1}\right)^{2}} d x_{1}+\sum_{\substack{m=1 \\
m \neq q}}^{2} \int_{c^{(m)}}^{d^{(m)}} \frac{\Delta u_{3}^{(\mathrm{imp})}\left(x_{1}\right)}{\left(x_{1}-\xi_{1}\right)^{2}} d x_{1} \\
& +\sum_{m=1}^{2} \int_{c^{(m)}}^{d^{(m)}} \Delta u_{3}^{(\mathrm{imp})}\left(x_{1}\right) \Theta\left(x_{1}, \xi_{1}\right) d x_{1} \\
& =-\frac{1}{T} \sigma_{3}+\frac{1}{T} \delta^{(q 2)} k_{33} \Delta u_{3}^{(\mathrm{imp})}\left(\xi_{1}\right) \text { for } c^{(q)}<\xi_{1}<d^{(q)}(q=1,2)
\end{aligned}
$$


where $\delta^{(k 2)}$ is such that $\delta^{(12)}=0$ and $\delta^{(22)}=1$.

For inplane deformations, the hypersingular integro-differential equations are

$$
\begin{aligned}
& f_{c^{(q)}}^{d^{(q)}} \frac{\operatorname{Re}\left\{Q_{j r}\right\} \Delta u_{r}^{(\mathrm{imp})}\left(x_{1}\right)}{\left(x_{1}-\xi_{1}\right)^{2}} d x_{1}+\sum_{\substack{m=1 \\
m \neq q}}^{2} \int_{c^{(m)}}^{d^{(m)}} \frac{\operatorname{Re}\left\{Q_{j r}\right\} \Delta u_{r}^{(\mathrm{imp})}\left(x_{1}\right)}{\left(x_{1}-\xi_{1}\right)^{2}} d x_{1} \\
& +\sum_{m=1}^{M} \int_{c^{(m)}}^{d^{(m)}} \operatorname{Re}\left\{Q_{j r}\right\} \Delta u_{r}^{(\mathrm{imp})}\left(x_{1}\right) \Theta\left(x_{1}, \xi_{1}\right) d x_{1}-\operatorname{Im}\left\{\pi Q_{j r}\right\} \frac{d}{d \xi_{1}}\left[\Delta u_{r}^{(\mathrm{imp})}\left(\xi_{1}\right)\right] \\
& =-\sigma_{j}+\delta^{(q 2)} k_{j i} \Delta u_{i}^{(\mathrm{imp})}\left(\xi_{1}\right) \text { for } c^{(q)}<\xi_{1}<d^{(q)}(q=1,2) .
\end{aligned}
$$

For antiplane deformations, the hypersingular integral equations in (3.37) are to be solved together with (3.35) for the unknown function $\Delta u_{3}^{(\mathrm{imp})}\left(x_{1}\right)$ and the effective stiffness $k_{33}$. For inplane deformations, (3.38) are to be solved together with (3.36) for $\Delta u_{r}^{(1)}\left(x_{1}\right), \Delta u_{r}^{(2)}\left(x_{1}\right)$ and $k_{i j}$ by using two independent sets of prescribed loads $\left[\sigma_{1}, \sigma_{2}\right]$ on the interface. Note that two independent sets of prescribed loads are needed for the case of inplane deformations so that (3.36) gives us four independent equations to account for the effective stiffness coefficients $k_{11}, k_{12}, k_{21}$ and $k_{22}$.

\subsubsection{Periodic Model Involving Evenly Distributed Micro- cracks of Equal Length}

In this periodic model, all the micro-cracks on the interface between the two orthotropic half-spaces have equal length and are evenly distributed. Thus, for the mathematical formulation of the model, we may regard a period interval of the micro-cracked interface in Section 3.2 as containing one micro-crack $(M=$ 1). The micro-crack has the length $2 a$ and the tips $\left(a^{(1)}, 0\right)$ and $\left(b^{(1)}, 0\right)$ in the portion of the interface where $0<x_{1}<L, x_{2}=0$ are given by $a^{(1)}=\frac{L}{2}(1-\rho)$ 


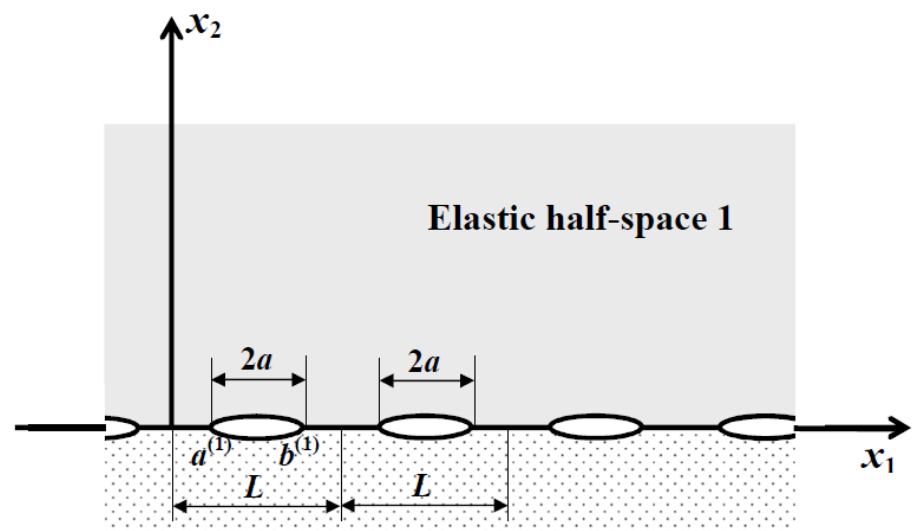

Elastic half - pace 2

Figure 3.3: A sketch of the periodic model.

and $b^{(1)}=a^{(1)}+2 a$. Note that $\rho=2 a / L$. The micro-cracks on the remaining parts of the interface are given by $a^{(1)}+n L<x_{1}<b^{(1)}+n L$ for $n= \pm 1, \pm 2$, $\cdots$, that is, micro-cracks of equal length $2 a$ are evenly distributed along the whole interface. For a sketch of the periodic model, refer to Figure 3.3.

For the model here, the hypersingular integro-differential equations (3.19) (for antiplane deformations) and (3.30) (for inplane deformations) can be respectively reduced to

$$
\begin{aligned}
& f_{a^{(1)}}^{b^{(1)}} \frac{\Delta u_{3}^{(\mathrm{imp})}\left(x_{1}\right)}{\left(x_{1}-\xi_{1}\right)^{2}} d x_{1}+\int_{a^{(1)}}^{b^{(1)}} \Delta u_{3}^{(\mathrm{imp})}\left(x_{1}\right) \Theta\left(x_{1}, \xi_{1}\right) d x_{1} \\
= & -\frac{1}{T} \sigma_{3} \text { for } a^{(1)}<\xi_{1}<b^{(1)}
\end{aligned}
$$


and

$$
\begin{aligned}
& f_{a^{(1)}}^{b^{(1)}} \frac{\operatorname{Re}\left\{Q_{j r}\right\} \Delta u_{r}^{(\mathrm{imp})}\left(x_{1}\right)}{\left(x_{1}-\xi_{1}\right)^{2}} d x_{1} \\
& +\int_{a^{(1)}}^{b^{(1)}} \operatorname{Re}\left\{Q_{j r}\right\} \Delta u_{r}^{(\mathrm{imp})}\left(x_{1}\right) \Theta\left(x_{1}, \xi_{1}\right) d x_{1}-\operatorname{Im}\left\{\pi Q_{j r}\right\} \frac{d \Delta u_{r}^{(\mathrm{imp})}\left(\xi_{1}\right)}{d \xi_{1}} \\
= & -\sigma_{j} \text { for } a^{(1)}<\xi_{1}<b^{(1)} .
\end{aligned}
$$

For antiplane deformations, once hypersingular integral equation in (3.39) is solved for $\Delta u_{3}^{(\mathrm{imp})}\left(x_{1}\right)$ for $a^{(1)}<x_{1}<b^{(1)}$, the effective stiffness $k_{33}$ can be computed using

$$
\frac{k_{33} \int_{a^{(1)}}^{b^{(1)}} \Delta u_{3}^{(\mathrm{imp})}\left(x_{1}\right) d x_{1}}{L}=\sigma_{3} .
$$

For inplane deformations, the hypersingular integro-differential equations (3.40) are solved for $\Delta u_{j}^{(\mathrm{imp})}\left(x_{1}\right)$ using two independent loads $\left[\sigma_{1}, \sigma_{2}\right]$. Denote the two independent loads by $\left[\sigma_{1}^{(1)}, \sigma_{2}^{(1)}\right]$ and $\left[\sigma_{1}^{(2)}, \sigma_{2}^{(2)}\right]$ (where $\left[\sigma_{1}^{(1)}, \sigma_{2}^{(1)}\right]$ is not a scalar multiple of $\left[\sigma_{1}^{(2)}, \sigma_{2}^{(2)}\right]$ ) and the corresponding displacement jumps $\Delta u_{j}^{(\mathrm{imp})}\left(x_{1}\right)$ by $\Delta u_{j}^{(1)}\left(x_{1}\right)$ and $\Delta u_{j}^{(2)}\left(x_{1}\right)$ respectively. The effective stiffness coefficients are then obtained by solving the following system of 4 linear algebraic equations in $k_{11}, k_{12}, k_{21}$ and $k_{22}$ :

$$
\begin{aligned}
& \frac{k_{i j} \int_{a^{(1)}}^{b^{(1)}} \Delta u_{j}^{(1)}\left(x_{1}\right) d x_{1}}{L}=\sigma_{i}^{(1)}, \\
& \frac{k_{i j} \int_{a^{(1)}}^{b^{(1)}} \Delta u_{j}^{(1)}\left(x_{1}\right) d x_{1}}{L}=\sigma_{i}^{(2)} .
\end{aligned}
$$

\subsubsection{Micromechanical-statistical Model}

The three-phase and the periodic models above assume that the micro-cracks on the interface of the bimaterial in Section 3.2 are of equal length and are 
evenly distributed on the interface. They do not take into consideration variations in the lengths of the micro-cracks. In the micromechanical-statistical model here, micro-cracks of varying lengths are statistically generated and randomly positioned on the interface.

To generate randomly an interface having a given damage ratio $\rho$, the lengths of $M$ micro-cracks are randomly generated to follow the chi-square distribution $\chi^{2}(m)$ ( $m$ is the degree of freedom) as explained in Section 2.7. The micro-cracks are then positioned randomly on the period interval $0<x_{1}<$ $L, x_{2}=0$. The micro-cracks on this period interval are duplicated periodically throughout the whole interface. If the average half micro-crack-length of the randomly generated micro-cracks is given by

$$
\widehat{a}=\frac{1}{2 M} \sum_{m=1}^{M}\left(b^{(m)}-a^{(m)}\right),
$$

then (3.2) gives rise to the relation $\rho L=2 M \widehat{a}$. Note that both sides of the relation denotes the total length of the damaged regions over a period length of the interface. Thus for a fixed damage ratio $\rho$, the period length $L$ may be calculated easily once the $M$ micro-cracks are randomly generated and positioned.

For fixed values of the material constants in the two orthotropic half-spaces, the average length of the micro-cracks, the damage ratio $\rho$ and the number of micro-cracks on a period length of the interface, $N$ interfaces are randomly generated as described above to form a statistical sample. The randomly generated interfaces in the sample are used to estimate the effective stiffness coefficients as follows.

For antiplane deformations, the hypersingular integral equations (3.19) with the load $\sigma_{32}^{(\text {ext) }}\left(\xi_{1}, 0^{+}\right)=\sigma_{3}$ (constant) are solved for the displacement jumps $\Delta u_{3}^{(\mathrm{imp})}\left(x_{1}\right)$ over the micro-cracks on each interface in the sample. The 


\section{Micromechanical Models for Weak Interfaces}

effective stiffness coefficient of the interface under consideration is then estimated by using

$$
\frac{k_{33} \sum_{m=1}^{M} \int_{a^{(m)}}^{b^{(m)}} \Delta u_{3}^{(\mathrm{imp})}\left(x_{1}\right) d x_{1}}{L}=\sigma_{3} .
$$

A set of $N$ data for the stiffness coefficient $k_{33}$ is obtained and processed statistically to estimate the antiplane effective stiffness of the micro-cracked interface.

Similarly, a set of $N$ data can be obtained for each of the inplane stiffness coefficients $k_{i j}$ by solving the hypersingular integro-differential equations in (3.30) for the $N$ interfaces. The hypersingular integro-differential equations in (3.30) are solved using two independent sets of loads given by $\sigma_{j 2}^{(\text {ext })}\left(\xi_{1}, 0^{+}\right)=\sigma_{j}^{(p)}$ (constant) $(j, p=1,2)$. The inplane stiffness coefficients are then determined by solving the system

$$
\frac{k_{i j} \sum_{m=1}^{M} \int_{a^{(m)}}^{b^{(m)}} \Delta u_{j}^{(p)}\left(x_{1}\right) d x_{1}}{L}=\sigma_{i}^{(p)} .
$$

Note that $\Delta u_{j}^{(p)}\left(x_{1}\right)$ are the displacement jumps $\Delta u_{j}^{(\mathrm{imp})}\left(x_{1}\right)$ for $\sigma_{j 2}^{(\mathrm{ext})}\left(\xi_{1}, 0^{+}\right)=$ $\sigma_{j}^{(p)}$.

If the appropriately non-dimensionalized values of the antiplane effective stiffness coefficient $k_{33}$ computed using the sample of the $N$ interfaces are given by $K_{33}^{(1)}, K_{33}^{(2)}, \ldots, K_{33}^{(N-1)}$ and $K_{33}^{(N)}$, then the mean value $\widehat{K}_{33}$ and the corresponding standard deviation $s_{33}$ of the non-dimensionalized effective stiffness coefficient are respectively given by

$$
\widehat{K}_{33}=\frac{1}{N} \sum_{n=1}^{N} K_{33}^{(n)} \text { and } s_{33}=\sqrt{\frac{1}{N-1} \sum_{n=1}^{N}\left(K_{33}^{(n)}-\widehat{K}_{33}\right)^{2}} .
$$

Similarly, if the appropriately non-dimensionalized values of the inplane effective stiffness coefficients $k_{i j}$ of the $N$ interfaces are given by $K_{i j}^{(1)}, K_{i j}^{(2)}, \ldots$, 
$K_{i j}^{(N-1)}$ and $K_{i j}^{(N)}$, then the mean value $\widehat{K}_{i j}$ and the corresponding standard deviation $s_{i j}$ of the non-dimensionalized effective stiffness coefficients are respectively given by

$$
\widehat{K}_{i j}=\frac{1}{N} \sum_{n=1}^{N} K_{i j}^{(n)} \text { and } s_{i j}=\sqrt{\frac{1}{N-1} \sum_{n=1}^{N}\left(K_{i j}^{(n)}-\widehat{K}_{i j}\right)^{2}} .
$$

\subsection{Numerical Procedures}

Numerical procedures for solving the hypersingular integro-differential equations for the three models in Section 3.4 are outlined here. The hypersingular integro-differential equations are eventually approximated by a system of linear algebraic equations.

\subsubsection{Three-phase Model}

For the three-phase model, the hypersingular integro-differential equations (3.37) together with (3.35) (for antiplane deformations) and (3.38) together with (3.36) (for inplane deformations) are solved by the numerical procedures below.

For antiplane deformations, the displacement jump $\Delta u_{3}^{(\text {imp })}\left(x_{1}\right)$ over $c^{(1)} \leq$ $x_{1} \leq d^{(1)}$ varies as $\sqrt{x_{1}-c^{(1)}}$ and $\sqrt{d^{(1)}-x_{1}}$ near $x_{1}=c^{(1)}$ and $x_{1}=d^{(1)}$ respectively. It is approximated as in Kaya and Erdogan [51] by using

$$
\begin{aligned}
\Delta u_{3}^{(\mathrm{imp})}\left(x_{1}\right) \simeq & \sqrt{\left(x_{1}-c^{(1)}\right)\left(d^{(1)}-x_{1}\right)} \\
& \times \sum_{m=1}^{N_{\mathrm{c}}} \alpha_{3}^{(m)} U^{(m-1)}\left(\frac{2 x_{1}-d^{(1)}-c^{(1)}}{d^{(1)}-c^{(1)}}\right) \\
& \text { for } c^{(1)}<x_{1}<d^{(1)},
\end{aligned}
$$

where $N_{\mathrm{c}}$ is a positive integer, $\alpha_{3}^{(1)}, a_{3}^{(2)}, \cdots, \alpha_{3}^{\left(N_{\mathrm{c}}-1\right)}$ and $\alpha_{3}^{\left(N_{\mathrm{c}}\right)}$ are constant coefficients to be determined and $U^{(m)}(x)$ is the $m$-th order Chebyshev poly- 
nomials of the second kind. Note that $N_{\mathrm{c}}$ may be required to be sufficiently large for (3.48) to be a good approximation. Details on such an approximation may be found in Ang [9].

For approximating $\Delta u_{3}^{(\mathrm{imp})}\left(x_{1}\right)$ over the effective region, the interval $c^{(2)}<$ $x_{1}<d^{(2)}$ is divided into $N_{\mathrm{e}}$ subintervals or elements given by

$$
x_{\mathrm{e}}^{(p)} \leq x_{1} \leq x_{\mathrm{e}}^{(p+1)}\left(p=1,2, \cdots, N_{\mathrm{e}}\right)
$$

where

$$
\begin{gathered}
x_{\mathrm{e}}^{(p)}=\frac{d^{(2)}+c^{(2)}}{2}-\frac{d^{(2)}-c^{(2)}}{2} \cos \left(\frac{[p-1] \pi}{N_{\mathrm{e}}}\right) \\
\quad \text { for } p=1,2, \ldots ., N_{\mathrm{e}}+1 .
\end{gathered}
$$

Note that (3.50) gives rise to more elements of shorter lengths nearer to the tips of the effective region. The displacement jumps $\Delta u_{3}^{(\mathrm{imp})}\left(x_{1}\right)$ over the $p$-th element on the effective region are approximated by

$$
\Delta u_{3}^{(\mathrm{imp})}\left(x_{1}\right) \simeq x_{1} \vartheta_{3}^{(p)}+\eta_{3}^{(p)} \text { for } x_{\mathrm{e}}^{(p)} \leq x_{1} \leq x_{\mathrm{e}}^{(p+1)},
$$

where $\vartheta_{3}^{(p)}$ and $\eta_{3}^{(p)}$ are $2 N_{\mathrm{e}}$ unknown constants yet to be determined.

With the approximations above, if (3.37) for $c^{(1)}<\xi_{1}<d^{(1)}$ are collocated at $N_{\text {c }}$ points (on the representative micro-crack) given by

$$
\begin{gathered}
\left(\widetilde{x}_{\mathrm{c}}^{(m)}, 0\right)=\left(\frac{d^{(1)}+c^{(1)}}{2}+\frac{d^{(1)}-c^{(1)}}{2} \cos \left(\frac{[2 m-1] \pi}{2 N_{\mathrm{c}}}\right), 0\right) \\
\quad \text { for } m=1,2, \cdots, N_{\mathrm{c}},
\end{gathered}
$$

then the hypersingular integral equations may be approximated by the linear algebraic equations 


$$
\begin{aligned}
& -\pi \sum_{m=1}^{N_{\mathrm{c}}} \alpha_{3}^{(m)} m U^{(m-1)}\left(\frac{2 \widetilde{x}_{\mathrm{c}}^{(j)}-d^{(1)}-c^{(1)}}{d^{(1)}-c^{(1)}}\right) \\
& +\sum_{m=1}^{N_{\mathrm{c}}} \alpha_{3}^{(m)} \int_{c^{(1)}}^{d^{(1)}} \sqrt{\left(x_{1}-c^{(1)}\right)\left(d^{(1)}-x_{1}\right)} \\
& \times U^{(m-1)}\left(\frac{2 x_{1}-d^{(1)}-c^{(1)}}{d^{(1)}-c^{(1)}}\right) \Theta\left(x_{1}, \widetilde{x}_{\mathrm{c}}^{(j)}\right) d x_{1} \\
& +\sum_{p=1}^{N_{\mathrm{e}}} \vartheta_{3}^{(p)}\left[F_{0}\left(x_{\mathrm{e}}^{(p+1)}, \widetilde{x}_{\mathrm{c}}^{(j)}\right)-F_{0}\left(x_{\mathrm{e}}^{(p)}, \widetilde{x}_{\mathrm{c}}^{(j)}\right)\right] \\
& +\sum_{p=1}^{N_{\mathrm{e}}} \vartheta_{3}^{(p)} \int_{x_{\mathrm{e}}^{(p)}}^{x_{\mathrm{e}}^{(p+1)}} x_{1} \Theta\left(x_{1}, \widetilde{x}_{\mathrm{c}}^{(j)}\right) d x_{1}+\sum_{p=1}^{N_{\mathrm{e}}} \eta_{3}^{(p)} \int_{x_{\mathrm{e}}^{(p)}}^{x_{\mathrm{e}}^{(p+1)}} \Theta\left(x_{1}, \widetilde{x}_{\mathrm{c}}^{(j)}\right) d x_{1} \\
& +\sum_{p=1}^{N_{\mathrm{e}}} \eta_{3}^{(p)}\left(-\frac{1}{x_{\mathrm{e}}^{(p+1)}-\widetilde{x}_{\mathrm{c}}^{(j)}}+\frac{1}{x_{\mathrm{e}}^{(p)}-\widetilde{x}_{\mathrm{c}}^{(j)}}\right) \\
& =-\frac{1}{T} \sigma_{3} \text { for } j=1,2, \ldots, N_{\mathrm{c}},
\end{aligned}
$$

where $F_{0}(x, t)=\ln |x-t|-t /(x-t)$.

If (3.37) for $c^{(2)}<\xi_{1}<d^{(2)}$ is collocated at $2 N_{\mathrm{e}}$ points (on the effective region) given by

$$
\left.\begin{array}{rl}
\left(\widetilde{x}_{\mathrm{e}}^{(p)}, 0\right) & =\left(\frac{3}{4} x_{\mathrm{e}}^{(p)}+\frac{1}{4} x_{\mathrm{e}}^{(p+1)}, 0\right) \\
\left(\widetilde{x}_{\mathrm{e}}^{\left(p+N_{\mathrm{e}}\right)}, 0\right) & =\left(\frac{1}{4} x_{\mathrm{e}}^{(p)}+\frac{3}{4} x_{\mathrm{e}}^{(p+1)}, 0\right)
\end{array}\right\} \text { for } p=1, \ldots, N_{\mathrm{e}}
$$

then the hypersingular integral equations may be approximately reduced to the linear algebraic equations 


$$
\begin{aligned}
& \sum_{m=1}^{N_{\mathrm{c}}} \alpha_{3}^{(m)} \int_{c^{(1)}}^{d^{(1)}} \sqrt{\left(x_{1}-c^{(1)}\right)\left(d^{(1)}-x_{1}\right)} U^{(m-1)}\left(\frac{2 x_{1}-d^{(1)}-c^{(1)}}{d^{(1)}-c^{(1)}}\right) \\
& \times\left[\frac{1}{\left(x_{1}-\widetilde{x}_{\mathrm{e}}^{(j)}\right)^{2}}+\Theta\left(x_{1}, \widetilde{x}_{\mathrm{e}}^{(j)}\right)\right] d x_{1} \\
& +\sum_{p=1}^{N_{\mathrm{e}}} \vartheta_{3}^{(p)}\left[F_{0}\left(x_{\mathrm{e}}^{(p+1)}, \widetilde{x}_{\mathrm{e}}^{(j)}\right)-F_{0}\left(x_{\mathrm{e}}^{(p)}, \widetilde{x}_{\mathrm{e}}^{(j)}\right)-\frac{1}{T} k_{33} \widetilde{x}_{\mathrm{e}}^{(j)} g_{\mathrm{e}}^{(p j)}\right] \\
& +\sum_{p=1}^{N_{\mathrm{e}}} \vartheta_{3}^{(p)} \int_{x_{\mathrm{e}}^{(p)}}^{x_{\mathrm{e}}^{(p+1)}} x_{1} \Theta\left(x_{1}, \widetilde{x}_{\mathrm{e}}^{(j)}\right) d x_{1}+\sum_{p=1}^{N_{\mathrm{e}}} \eta_{3}^{(p)} \int_{x_{\mathrm{e}}^{(p)}}^{x_{\mathrm{e}}^{(p+1)}} \Theta\left(x_{1}, \widetilde{x}_{\mathrm{e}}^{(j)}\right) d x_{1} \\
& +\sum_{p=1}^{N_{\mathrm{e}}} \eta_{3}^{(p)}\left(-\frac{1}{x_{\mathrm{e}}^{(p+1)}-\widetilde{x}_{\mathrm{e}}^{(j)}}+\frac{1}{x_{\mathrm{e}}^{(p)}-\widetilde{x}_{\mathrm{e}}^{(j)}}-\frac{k_{33}}{T} g_{\mathrm{e}}^{(p j)}\right) \\
& =-\frac{1}{T} \sigma_{3} \text { for } j=1,2, \ldots, 2 N_{\mathrm{e}},
\end{aligned}
$$

where $g_{\mathrm{e}}^{(p j)}$ is given by

$$
g_{\mathrm{e}}^{(p j)}=\left\{\begin{array}{l}
1 \text { for } p=j \text { or } p=j+N_{\mathrm{e}} \\
0 \text { otherwise }
\end{array}\right.
$$

Substitution of (3.48) into (3.35) gives rise to

$$
\begin{aligned}
k_{33}= & \sigma_{3} c^{(2)}\left[\sum_{m=1}^{N_{\mathrm{c}}} \alpha_{3}^{(m)} \int_{c^{(1)}}^{d^{(1)}} \sqrt{\left(x_{1}-c^{(1)}\right)\left(d^{(1)}-x_{1}\right)}\right. \\
& \left.\times U^{(m-1)}\left(\frac{2 x_{1}-d^{(1)}-c^{(1)}}{d^{(1)}-c^{(1)}}\right) d x_{1}\right]^{-1} .
\end{aligned}
$$

The equations in (3.53) and (3.55) may be solved for the unknowns $\alpha_{3}^{(m)}$ $\left(m=1,2, \ldots, N_{\mathrm{c}}\right), \vartheta_{3}^{(p)}\left(p=1,2, \ldots, N_{\mathrm{e}}\right), \eta_{3}^{(p)}\left(p=1,2, \ldots, N_{\mathrm{e}}\right)$ and $k_{33}$. An iterative method may be used to solve the equations as follows. With a guessed value of $k_{33},(3.53)$ and (3.55) are solved as a system of $N_{\mathrm{c}}+2 N_{\mathrm{e}}$ linear algebraic equations for $\alpha_{3}^{(m)}, \vartheta_{3}^{(p)}$ and $\eta_{3}^{(p)}$. The value of $k_{33}$ is then updated using (3.57). With the updated value of $k_{33},(3.53)$ and (3.55) are solved again. The 
procedure is repeated until $k_{33}$ converges to a prescribed number of significant figures. For the range of the parameters used in the calculations in Sections 3.6 and 3.7, the effective stiffness $k_{33}$ is found to converge to at least two significant figures in less than six iterations.

Note that all the integrals in (3.53) and (3.55) may be evaluated accurately by using suitable integration quadratures. Specifically, the integrals containing the term $\sqrt{\left(x_{1}-c^{(1)}\right)\left(d^{(1)}-x_{1}\right)}$ in the integrands may be computed with good accuracy by using formula (25.4.40) in Abramowitz and Stegun [5] and the other integrals by the Simpson's rule.

For inplane deformations, the displacement jumps $\Delta u_{r}^{(\mathrm{imp})}\left(x_{1}\right)$ for $c^{(1)}<$ $x_{1}<d^{(1)}$ (over the representative micro-crack in the three-phase model) are approximated by

$$
\begin{aligned}
\Delta u_{r}^{(\mathrm{imp})}\left(x_{1}\right) \simeq & \sqrt{\left(x_{1}-c^{(1)}\right)\left(d^{(1)}-x_{1}\right)} \\
& \times \sum_{m=1}^{N_{\mathrm{c}}} \alpha_{r}^{(m)} U^{(m-1)}\left(\frac{2 x_{1}-d^{(1)}-c^{(1)}}{d^{(1)}-c^{(1)}}\right) \\
& \text { for } c^{(1)}<x_{1}<d^{(1)},
\end{aligned}
$$

where $\alpha_{r}^{(m)}$ are $2 N_{\mathrm{c}}$ unknown constants to be determined.

As given in (3.49) and (3.50), the effective region on $c^{(2)}<x_{1}<d^{(2)}$ is divided into $N_{\mathrm{e}}$ elements. The displacement jumps $\Delta u_{r}^{(\mathrm{imp})}\left(x_{1}\right)$ over the $p$-th element on the effective region are approximated by

$$
\Delta u_{r}^{(\mathrm{imp})}\left(x_{1}\right) \simeq x_{1} \vartheta_{r}^{(p)}+\eta_{r}^{(p)} \text { for } x_{\mathrm{e}}^{(p)} \leq x_{1} \leq x_{\mathrm{e}}^{(p+1)},
$$

where $\vartheta_{r}^{(p)}$ and $\eta_{r}^{(p)}$ are $4 N_{\mathrm{e}}$ unknown constants yet to be determined.

As in the case for antiplane deformations, using the approximations (3.58) and (3.59) in the hypersingular integro-differential equations (3.38) for $c^{(1)}<$ $x_{1}<d^{(1)}$ and collocating the resulting equations at the $N_{\mathrm{c}}$ points given in (3.52) 
on the representative micro-crack give rise to the linear algebraic equations

$$
\begin{aligned}
& -\pi \sum_{m=1}^{N_{\mathrm{c}}} \alpha_{r}^{(m)} \operatorname{Re}\left\{Q_{j r}\right\} m U^{(m-1)}\left(\frac{2 \widetilde{x}_{\mathrm{c}}^{(n)}-d^{(1)}-c^{(1)}}{d^{(1)}-c^{(1)}}\right) \\
& +\sum_{m=1}^{N_{\mathrm{c}}} \alpha_{r}^{(m)} \operatorname{Re}\left\{Q_{j r}\right\} \int_{c^{(1)}}^{d^{(1)}} \sqrt{\left(x_{1}-c^{(1)}\right)\left(d^{(1)}-x_{1}\right)} \\
& \times U^{(m-1)}\left(\frac{2 x_{1}-d^{(1)}-c^{(1)}}{d^{(1)}-c^{(1)}}\right) \Theta\left(x_{1}, \widetilde{x}_{\mathrm{c}}^{(n)}\right) d x_{1} \\
& -\sum_{m=1}^{N_{c}} \alpha_{r}^{(m)} \operatorname{Im}\left\{\pi Q_{j r}\right\} \frac{\left(c^{(1)}-d^{(1)}\right) m \cos \left[m \arccos \left(\frac{2 \widetilde{x}_{\mathrm{c}}^{(n)}-d^{(1)}-c^{(1)}}{d^{(1)}-c^{(1)}}\right)\right]}{2 \sqrt{\left(\widetilde{x}_{\mathrm{c}}^{(n)}-c^{(1)}\right)\left(d^{(1)}-\widetilde{x}_{\mathrm{c}}^{(n)}\right)}} \\
& +\sum_{p=1}^{N_{\mathrm{e}}} \vartheta_{r}^{(p)} \operatorname{Re}\left\{Q_{j r}\right\}\left[F_{0}\left(x_{\mathrm{e}}^{(p+1)}, \widetilde{x}_{\mathrm{c}}^{(n)}\right)-F_{0}\left(x_{\mathrm{e}}^{(p)}, \widetilde{x}_{\mathrm{c}}^{(n)}\right)\right] \\
& +\sum_{p=1}^{N_{\mathrm{e}}} \vartheta_{r}^{(p)} \operatorname{Re}\left\{Q_{j r}\right\} \int_{x_{\mathrm{e}}^{(p)}}^{x_{\mathrm{e}}^{(p+1)}} x_{1} \Theta\left(x_{1}, \widetilde{x}_{\mathrm{c}}^{(n)}\right) d x_{1} \\
& +\sum_{p=1}^{N_{\mathrm{e}}} \eta_{r}^{(p)} \operatorname{Re}\left\{Q_{j r}\right\}\left(-\frac{1}{x_{\mathrm{e}}^{(p+1)}-\widetilde{x}_{\mathrm{c}}^{(n)}}+\frac{1}{x_{\mathrm{e}}^{(p)}-\widetilde{x}_{\mathrm{c}}^{(n)}}\right) \\
& +\sum_{p=1}^{N_{\mathrm{e}}} \eta_{r}^{(p)} \operatorname{Re}\left\{Q_{j r}\right\} \int_{x_{\mathrm{e}}^{(p)}}^{x_{\mathrm{e}}^{(p+1)}} \Theta\left(x_{1}, \widetilde{x}_{\mathrm{c}}^{(n)}\right) d x_{1} \\
& =-\sigma_{j} \text { for } n=1,2, \ldots, N_{\mathrm{c}} .
\end{aligned}
$$

Also, the hypersingular integro-differential equations (3.38) for $c^{(2)}<x_{1}<d^{(2)}$ are collocated at the $N_{\mathrm{e}}$ points given in (3.54) on the effective region to obtain the linear algebraic equations 


$$
\begin{aligned}
& \sum_{m=1}^{N_{\mathrm{c}}} \alpha_{r}^{(m)} \operatorname{Re}\left\{Q_{j r}\right\} \int_{c^{(1)}}^{d^{(1)}} \sqrt{\left(x_{1}-c^{(1)}\right)\left(d^{(1)}-x_{1}\right)} U^{(m-1)}\left(\frac{2 x_{1}-d^{(1)}-c^{(1)}}{d^{(1)}-c^{(1)}}\right) \\
& \times\left[\frac{1}{\left(x_{1}-\widetilde{x}_{\mathrm{e}}^{(n)}\right)^{2}}+\Theta\left(x_{1}, \widetilde{x}_{\mathrm{e}}^{(n)}\right)\right] d x_{1} \\
& +\sum_{p=1}^{N_{\mathrm{e}}} \vartheta_{r}^{(p)}\left[\operatorname{Re}\left\{Q_{j r}\right\}\left(F_{0}\left(x_{\mathrm{e}}^{(p+1)}, \widetilde{x}_{\mathrm{e}}^{(n)}\right)-F_{0}\left(x_{\mathrm{e}}^{(p)}, \widetilde{x}_{\mathrm{e}}^{(n)}\right)\right)\right. \\
& \left.-\left(\operatorname{Im}\left\{\pi Q_{j r}\right\}+k_{j r} \widetilde{x}_{\mathrm{e}}^{(n)}\right) g_{\mathrm{e}}^{(p n)}\right] \\
& +\sum_{p=1}^{N_{\mathrm{e}}} \vartheta_{r}^{(p)} \operatorname{Re}\left\{Q_{j r}\right\} \int_{x_{\mathrm{e}}^{(p)}}^{x_{\mathrm{e}}^{(p+1)}} x_{1} \Theta\left(x_{1}, \widetilde{x}_{\mathrm{e}}^{(n)}\right) d x_{1} \\
& +\sum_{p=1}^{N_{\mathrm{e}}} \eta_{r}^{(p)}\left[\operatorname{Re}\left\{Q_{j r}\right\}\left(-\frac{1}{x_{\mathrm{e}}^{(p+1)}-\widetilde{x}_{\mathrm{e}}^{(n)}}+\frac{1}{x_{\mathrm{e}}^{(p)}-\widetilde{x}_{\mathrm{e}}^{(n)}}\right)-k_{j r} g_{\mathrm{e}}^{(p n)}\right] \\
& +\sum_{p=1}^{N_{\mathrm{e}}} \eta_{r}^{(p)} \operatorname{Re}\left\{Q_{j r}\right\} \int_{x_{\mathrm{e}}^{(p)}}^{x_{\mathrm{e}}^{(p+1)}} \Theta\left(x_{1}, \widetilde{x}_{\mathrm{e}}^{(n)}\right) d x_{1} \\
& =-\sigma_{j} \text { for } n=1,2, \ldots, 2 N_{\mathrm{e}} .
\end{aligned}
$$

If the two independent loads on the micro-cracks and the effective regions are given by $\left[\sigma_{1}^{(1)}, \sigma_{2}^{(1)}\right]$ and $\left[\sigma_{1}^{(2)}, \sigma_{2}^{(2)}\right]$ and the constants $\alpha_{j}^{(m)}$ in the corresponding displacement jumps $\Delta u_{j}^{(\mathrm{imp})}\left(x_{1}\right)$ are given by $\alpha_{j}^{(1 m)}$ and $\alpha_{j}^{(2 m)}$, then (3.36) can be approximately written as

$$
\begin{array}{r}
k_{i j} \sum_{m=1}^{N_{c}} \alpha_{j}^{(p m)} \int_{c^{(1)}}^{d^{(1)}} \sqrt{\left(x_{1}-c^{(1)}\right)\left(d^{(1)}-x_{1}\right)} \\
\times U^{(m-1)}\left(\frac{2 x_{1}-d^{(1)}-c^{(1)}}{d^{(1)}-c^{(1)}}\right) d x_{1}=c^{(2)} \sigma_{i}^{(p)} \\
\text { for } p=1,2 .
\end{array}
$$

As in the case for antiplane deformations, an iterative procedure may be used to solve (3.60), (3.61) and (3.62) for $\alpha_{j}^{(p m)}$ and the inplane effective stiffness coefficients $k_{i j}$. If $k_{i j}$ are assumed known, the linear algebraic equations 
(3.60) and (3.61) under the loads $\left[\sigma_{1}^{(p)}, \sigma_{2}^{(p)}\right]$ may be solved for the constants $\alpha_{j}^{(p m)}\left(m=1,2, \ldots, N_{\mathrm{c}}\right)$ in (3.58) and $\vartheta_{r}^{(q)}$ and $\eta_{r}^{(q)}\left(q=1,2, \ldots, N_{\mathrm{e}}\right)$ in (3.59). Once $\alpha_{j}^{(p m)}$ are determined, we may update the values of $k_{i j}$ by using (3.62). The linear algebraic equations are then solved again using the updated values of $k_{i j}$. The iteration between solving the linear algebraic equations and updating the values of $k_{i j}$ is repeated until the values of $k_{i j}$ converge to within a prescribed number of significant figures.

\subsubsection{Periodic Model Involving Evenly Distributed Micro- cracks of Equal Length}

For the periodic model under antiplane deformations, the hypersingular integrodifferential equations (3.39) together with (3.41) may be solved by the numerical procedures (3.63)-(3.66) below.

As in (3.48), the displacement jump $\Delta u_{3}^{(\mathrm{imp})}\left(x_{1}\right)$ over the micro-crack on $a^{(1)}<x_{1}<b^{(1)}$ is approximated by using

$$
\begin{aligned}
\Delta u_{3}^{(\mathrm{imp})}\left(x_{1}\right) \simeq & \sqrt{\left(x_{1}-a^{(1)}\right)\left(b^{(1)}-x_{1}\right)} \\
& \times \sum_{m=1}^{N_{\mathrm{c}}} \alpha_{3}^{(m)} U^{(m-1)}\left(\frac{2 x_{1}-b^{(1)}-a^{(1)}}{b^{(1)}-a^{(1)}}\right) \\
& \text { for } a^{(1)}<x_{1}<b^{(1)} .
\end{aligned}
$$

With the approximations in (3.63), if (3.39) are collocated on $N_{\mathrm{c}}$ points (over the micro-crack) given by

$$
\begin{gathered}
\left(\widetilde{x}_{\mathrm{c}}^{(m)}, 0\right)=\left(\frac{b^{(1)}+a^{(1)}}{2}+\frac{b^{(1)}-a^{(1)}}{2} \cos \left(\frac{[2 m-1] \pi}{2 N_{\mathrm{c}}}\right), 0\right) \\
\quad \text { for } m=1,2, \cdots, N_{\mathrm{c}},
\end{gathered}
$$


then (3.39) may be reduced to the linear algebraic equations

$$
\begin{aligned}
& -\sum_{m=1}^{N_{\mathrm{c}}} \alpha_{3}^{(m)} \pi m U^{(m-1)}\left(\frac{2 \widetilde{x}_{\mathrm{c}}^{(j)}-b^{(1)}-a^{(1)}}{b^{(1)}-a^{(1)}}\right) \\
& +\sum_{m=1}^{N_{\mathrm{c}}} \alpha_{3}^{(m)} \int_{a^{(1)}}^{b^{(1)}} \sqrt{\left(x_{1}-a^{(1)}\right)\left(b^{(1)}-x_{1}\right)} \\
& \times U^{(m-1)}\left(\frac{2 x_{1}-b^{(1)}-a^{(1)}}{b^{(1)}-a^{(1)}}\right) \Theta\left(x_{1}, \widetilde{x}_{\mathrm{c}}^{(j)}\right) d x_{1} \\
= & -\frac{1}{T} \sigma_{3} \text { for } j=1,2, \ldots, N_{\mathrm{c}} .
\end{aligned}
$$

Once the constants $\alpha_{3}^{(m)}\left(m=1,2, \ldots, N_{\mathrm{c}}\right)$ are determined by solving the linear algebraic equations (3.65), we may estimate the effective stiffness $k_{33}$ simultaneously by using

$$
\begin{aligned}
k_{33}= & \sigma_{3} L\left[\sum_{m=1}^{N_{\mathrm{c}}} \alpha_{3}^{(m)} \int_{a^{(1)}}^{b^{(1)}} \sqrt{\left(x_{1}-a^{(1)}\right)\left(b^{(1)}-x_{1}\right)} d x_{1}\right. \\
& \left.\times U^{(m-1)}\left(\frac{2 x_{1}-b^{(1)}-a^{(1)}}{b^{(1)}-a^{(1)}}\right)\right]^{-1} .
\end{aligned}
$$

For inplane deformations, the numerical procedure used for solving the hypersingular integro-differential equations (3.40) together with (3.42) is outlined below.

As in (3.58), the displacement jumps $\Delta u_{r}^{(\mathrm{imp})}\left(x_{1}\right)$ over the micro-crack are approximated by

$$
\begin{aligned}
\Delta u_{r}^{(\mathrm{imp})}\left(x_{1}\right) \simeq & \sqrt{\left(x_{1}-a^{(1)}\right)\left(b^{(1)}-x_{1}\right)} \\
& \times \sum_{m=1}^{N_{c}} \alpha_{r}^{(m)} U^{(m-1)}\left(\frac{2 x_{1}-b^{(1)}-a^{(1)}}{b^{(1)}-a^{(1)}}\right) \\
& \text { for } a^{(1)}<x_{1}<b^{(1)} .
\end{aligned}
$$

By substituting (3.67) into the hypersingular integro-differential equations (3.40) and collocating the resulting equations on the $N_{\mathrm{c}}$ points given in (3.64), 
we may reduce (3.40) to the linear algebraic equations

$$
\begin{aligned}
& -\sum_{m=1}^{N_{\mathrm{c}}} \alpha_{r}^{(m)} \operatorname{Re}\left\{Q_{j r}\right\} \pi m U^{(m-1)}\left(\frac{2 \widetilde{x}_{\mathrm{c}}^{(n)}-b^{(1)}-a^{(1)}}{b^{(1)}-a^{(1)}}\right) \\
& +\sum_{m=1}^{N_{\mathrm{c}}} \alpha_{r}^{(m)} \operatorname{Re}\left\{Q_{j r}\right\} \int_{a^{(1)}}^{b^{(1)}} \sqrt{\left(x_{1}-a^{(1)}\right)\left(b^{(1)}-x_{1}\right)} \\
& \times U^{(m-1)}\left(\frac{2 x_{1}-b^{(1)}-a^{(1)}}{b^{(1)}-a^{(1)}}\right) \Theta\left(x_{1}, \widetilde{x}_{\mathrm{c}}^{(n)}\right) d x_{1} \\
& -\sum_{m=1}^{N_{c}} \alpha_{r}^{(m)} \operatorname{Im}\left\{\pi Q_{j r}\right\} \frac{\left(a^{(1)}-b^{(1)}\right) m \cos \left[m \arccos \left(\frac{2 \widetilde{x}_{\mathrm{c}}^{(n)}-b^{(1)}-a^{(1)}}{b^{(1)}-a^{(1)}}\right)\right]}{2 \sqrt{\left(\widetilde{x}_{\mathrm{c}}^{(n)}-a^{(1)}\right)\left(b^{(1)}-\widetilde{x}_{\mathrm{c}}^{(n)}\right)}} \\
& =-\sigma_{j} \text { for } n=1,2, \ldots, N_{\mathrm{c}} .
\end{aligned}
$$

Once the linear algebraic equations (3.68) are solved using the two inde-

pendent loads $\left[\sigma_{1}^{(1)}, \sigma_{2}^{(1)}\right]$ and $\left[\sigma_{1}^{(2)}, \sigma_{2}^{(2)}\right]$, the effective stiffness coefficients $k_{i j}$ may be calculated simultaneously by using

$$
\begin{gathered}
k_{i j} \sum_{m=1}^{N_{\mathrm{c}}} \alpha_{j}^{(p m)} \int_{a^{(1)}}^{b^{(1)}} \sqrt{\left(x_{1}-a^{(1)}\right)\left(b^{(1)}-x_{1}\right)} \\
\times U^{(m-1)}\left(\frac{2 x_{1}-b^{(1)}-a^{(1)}}{b^{(1)}-a^{(1)}}\right) d x_{1}=\sigma_{i}^{(p)} L \\
\text { for } p=1,2 .
\end{gathered}
$$

Note that $\alpha_{j}^{(p m)}$ are the constants $\alpha_{j}^{(m)}$ in (3.68) for $\sigma_{i}=\sigma_{i}^{(p)}$.

\subsubsection{Micromechanical-statistical Model}

For the micromechanical-statistical model, the hypersingular integro-differential equations (3.19) together with (3.44) (for antiplane deformations) and (3.30) together with (3.45) (for inplane deformations) are solved by using the numerical procedures below. 
For antiplane deformations, the displacement jump $\Delta u_{3}^{(\mathrm{imp})}\left(x_{1}\right)$ over the $k$-th micro-crack on the interval $0<x_{1}<L, x_{2}=0$ is approximated by using

$$
\begin{aligned}
\Delta u_{3}^{(\mathrm{imp})}\left(x_{1}\right) \simeq & \sqrt{\left(x_{1}-a^{(k)}\right)\left(b^{(k)}-x_{1}\right)} \\
& \times \sum_{m=1}^{N_{c}^{(k)}} \alpha_{3}^{(k m)} U^{(m-1)}\left(\frac{2 x_{1}-b^{(k)}-a^{(k)}}{b^{(k)}-a^{(k)}}\right) \\
& \text { for } a^{(k)}<x_{1}<b^{(k)}(k=1,2, \ldots, M),
\end{aligned}
$$

where $\alpha_{3}^{(k m)}$ are unknown constants to be determined and $N_{\mathrm{c}}^{(k)}$ are positive integers. By substituting (3.70) into (3.19) and collocating the resulting equations on the collocation points given by

$$
\begin{array}{r}
\left(\widetilde{x}_{\mathrm{c}}^{(k p)}, 0\right)=\left(\frac{b^{(k)}+a^{(k)}}{2}+\frac{b^{(k)}-a^{(k)}}{2} \cos \left(\frac{[2 p-1] \pi}{2 N_{\mathrm{c}}^{(k)}}\right), 0\right) \\
\quad \text { for } p=1,2, \cdots, N_{\mathrm{c}}^{(k)}(k=1,2, \ldots, M),
\end{array}
$$

we may reduce the hypersingular integro-differential equations (3.19) to the linear algebraic equations

$$
\begin{aligned}
& -\pi \sum_{m=1}^{N_{\mathrm{c}}^{(k)}} \alpha_{3}^{(k m)} m U^{(m-1)}\left(\frac{2 \widetilde{x}_{\mathrm{c}}^{(k p)}-b^{(k)}-a^{(k)}}{b^{(k)}-a^{(k)}}\right) \\
& +\sum_{\substack{n=1 \\
n \neq k}}^{M} \sum_{m=1}^{N_{\mathrm{c}}^{(n)}} \alpha_{3}^{(n m)} \int_{a^{(n)}}^{b^{(n)}} \frac{\sqrt{\left(x_{1}-a^{(n)}\right)\left(b^{(n)}-x_{1}\right)}}{\left(x_{1}-\widetilde{x}_{\mathrm{c}}^{(k p)}\right)^{2}} \\
& \times U^{(m-1)}\left(\frac{2 x_{1}-b^{(n)}-a^{(n)}}{b^{(n)}-a^{(n)}}\right) d x_{1} \\
& +\sum_{n=1}^{M} \sum_{m=1}^{N_{\mathrm{c}}^{(n)}} \alpha_{3}^{(n m)} \int_{a^{(n)}}^{b^{(n)}} \sqrt{\left(x_{1}-a^{(n)}\right)\left(b^{(n)}-x_{1}\right)} \\
& \times U^{(m-1)}\left(\frac{2 x_{1}-b^{(n)}-a^{(n)}}{b^{(n)}-a^{(n)}}\right) \Theta\left(x_{1}, \widetilde{x}_{\mathrm{c}}^{(k p)}\right) d x_{1} \\
& =-\frac{1}{T} \sigma_{3} \text { for } p=1,2, \ldots, N_{\mathrm{c}}^{(k)}(k=1,2, \ldots, M) .
\end{aligned}
$$


Once the constants $\alpha_{3}^{(n m)}$ are obtained by solving the linear algebraic equations (3.72), the effective stiffness $k_{33}$ may be estimated by substituting (3.70) into (3.44), that is, via the formula

$$
\begin{aligned}
k_{33}= & \sigma_{3} L\left[\sum_{k=1}^{M} \sum_{m=1}^{N_{c}^{(k)}} \alpha_{3}^{(k m)} \int_{a^{(k)}}^{b^{(k)}} \sqrt{\left(x_{1}-a^{(k)}\right)\left(b^{(k)}-x_{1}\right)}\right. \\
& \left.\times U^{(m-1)}\left(\frac{2 x_{1}-b^{(k)}-a^{(k)}}{b^{(k)}-a^{(k)}}\right)\right]^{-1}
\end{aligned}
$$

For inplane deformations, the displacement jumps $\Delta u_{r}^{(\mathrm{imp})}\left(x_{1}\right)$ over the micro-cracks are approximated by

$$
\begin{aligned}
\Delta u_{r}^{(\mathrm{imp})}\left(x_{1}\right) \simeq & \sqrt{\left(x_{1}-a^{(k)}\right)\left(b^{(k)}-x_{1}\right)} \\
& \times \sum_{m=1}^{N_{c}^{(k)}} \alpha_{r}^{(k m)} U^{(m-1)}\left(\frac{2 x_{1}-b^{(k)}-a^{(k)}}{b^{(k)}-a^{(k)}}\right) \\
& \text { for } a^{(k)}<x_{1}<b^{(k)}(k=1,2, \ldots, M) .
\end{aligned}
$$

Using the approximations (3.74) in (3.30) and collocating the resulting equations on the points given by (3.71) give rise to the linear algebraic equations 


$$
\begin{aligned}
& -\pi \sum_{m=1}^{N_{c}^{(k)}} \alpha_{r}^{(k m)} \operatorname{Re}\left\{Q_{j r}\right\} m U^{(m-1)}\left(\frac{2 \widetilde{x}_{\mathrm{c}}^{(k p)}-b^{(k)}-a^{(k)}}{b^{(k)}-a^{(k)}}\right) \\
& +\sum_{\substack{n=1 \\
n \neq k}}^{M} \sum_{m=1}^{N_{c}^{(n)}} \alpha_{r}^{(n m)} \operatorname{Re}\left\{Q_{j r}\right\} \int_{a^{(n)}}^{b^{(n)}} \frac{\sqrt{\left(x_{1}-a^{(n)}\right)\left(b^{(n)}-x_{1}\right)}}{\left(x_{1}-\widetilde{x}_{\mathrm{c}}^{(k p)}\right)^{2}} \\
& \times U^{(m-1)}\left(\frac{2 x_{1}-b^{(n)}-a^{(n)}}{b^{(n)}-a^{(n)}}\right) d x_{1} \\
& +\sum_{n=1}^{M} \sum_{m=1}^{N_{c}^{(n)}} \alpha_{r}^{(n m)} \operatorname{Re}\left\{Q_{j r}\right\} \int_{a^{(n)}}^{b^{(n)}} \sqrt{\left(x_{1}-a^{(n)}\right)\left(b^{(n)}-x_{1}\right)} \\
& \times U^{(m-1)}\left(\frac{2 x_{1}-b^{(n)}-a^{(n)}}{b^{(n)}-a^{(n)}}\right) \Theta\left(x_{1}, \widetilde{x}_{\mathrm{c}}^{(k p)}\right) d x_{1} \\
& -\sum_{m=1}^{N_{c}^{(k)}} \alpha_{r}^{(k m)} \operatorname{Im}\left\{\pi Q_{j r}\right\} \frac{\left(a^{(k)}-b^{(k)}\right) m \cos \left[m \arccos \left(\frac{2 \widetilde{x}_{\mathrm{c}}^{(k p)}-b^{(k)}-a^{(k)}}{b^{(k)}-a^{(k)}}\right)\right]}{2 \sqrt{\left(\widetilde{x}_{\mathrm{c}}^{(k p)}-a^{(k)}\right)\left(b^{(k)}-\widetilde{x}_{\mathrm{c}}^{(k p)}\right)}} \\
& =-\sigma_{j} \text { for } p=1,2, \ldots, N_{\mathrm{c}}^{(k)}(k=1,2, \ldots, M) .
\end{aligned}
$$

Once the linear algebraic equations (3.75) are solved by taking $\sigma_{j}=\sigma_{j}^{(p)}(p=$ $1,2)$, the effective stiffness coefficients $k_{i j}$ may be computed readily by using

$$
\begin{gathered}
k_{i j} \sum_{k=1}^{M} \sum_{m=1}^{N_{c}^{(k)}} \alpha_{j}^{(p k m)} \int_{a^{(k)}}^{b^{(k)}} \sqrt{\left(x_{1}-a^{(k)}\right)\left(b^{(k)}-x_{1}\right)} \\
\times U^{(m-1)}\left(\frac{2 x_{1}-b^{(k)}-a^{(k)}}{b^{(k)}-a^{(k)}}\right) d x_{1}=\sigma_{i}^{(p)} L \\
\text { for } p=1,2,
\end{gathered}
$$

where $\alpha_{j}^{(p k m)}$ are the constants $\alpha_{j}^{(k m)}$ in (3.75) for the loads $\sigma_{j}^{(p)}$.

\subsubsection{Numerical Values of Effective Stiffness Coefficients}

For all the three models described above, all the numerical values of the inplane effective stiffness coefficients obtained in Sections 3.6 and 3.7 show that $k_{11}$ and $k_{22}$ are comparable in magnitudes but $k_{12}$ and $k_{21}$ are insignificant in 
magnitude compared to either $k_{11}$ or $k_{22}$. Specifically, we find that $\left|k_{12} / k_{11}\right|$ and $\left|k_{21} / k_{22}\right|$ are of the order $10^{-9}$ or smaller. Thus, for all the three models here, $k_{12}$ and $k_{21}$ are regarded as insignificant for spring-like interfaces between two orthotropic elastic half-spaces. They are ignored in the numerical results presented in Sections 3.6 and 3.7 below.

\subsection{Numerical Comparison of the Three-Phase and the Periodic Models}

The values of the effective stiffness coefficients $k_{i j}$ (for inplane deformations) and $k_{33}$ (for antiplane deformations) computed by using the numerical procedures for the three-phase model in Subsection 3.5.1 are compared here with the corresponding ones calculated using the numerical procedures for the periodic model in Subsection 3.5.2. The two sets of values are expected to be close to each other.

\subsubsection{Antiplane Deformations}

For antiplane deformations, plots of the non-dimensionalized interface stiffness coefficient $a\left(\beta^{(1)}+\beta^{(2)}\right) k_{33} /\left(2 \beta^{(1)} \beta^{(2)}\right)$ against $\rho$ for $0.10 \leq \rho \leq 0.90$ are given in Figure 3.4. Note that $\beta^{(p)}=\sqrt{C_{44}^{(p)} C_{55}^{(p)}}$ and the non-dimensionalized effective stiffness $a\left(\beta^{(1)}+\beta^{(2)}\right) k_{33} /\left(2 \beta^{(1)} \beta^{(2)}\right)$ is independent of the material constants of the two half-spaces and is a function of only $\rho$. As expected, the plot obtained for the periodic model in Subsection 3.5.1 is close to the one for the corresponding three-phase model in Subsection 3.5.2. The percentage difference between the two plots for $a\left(\beta^{(1)}+\beta^{(2)}\right) k_{33} /\left(2 \beta^{(1)} \beta^{(2)}\right)$ ranges approximately from $0.25 \%(\rho=0.10)$ to $2.5 \%(\rho=0.90)$. As may be expected, the percentage difference is larger for $\rho$ closer to 1 . 


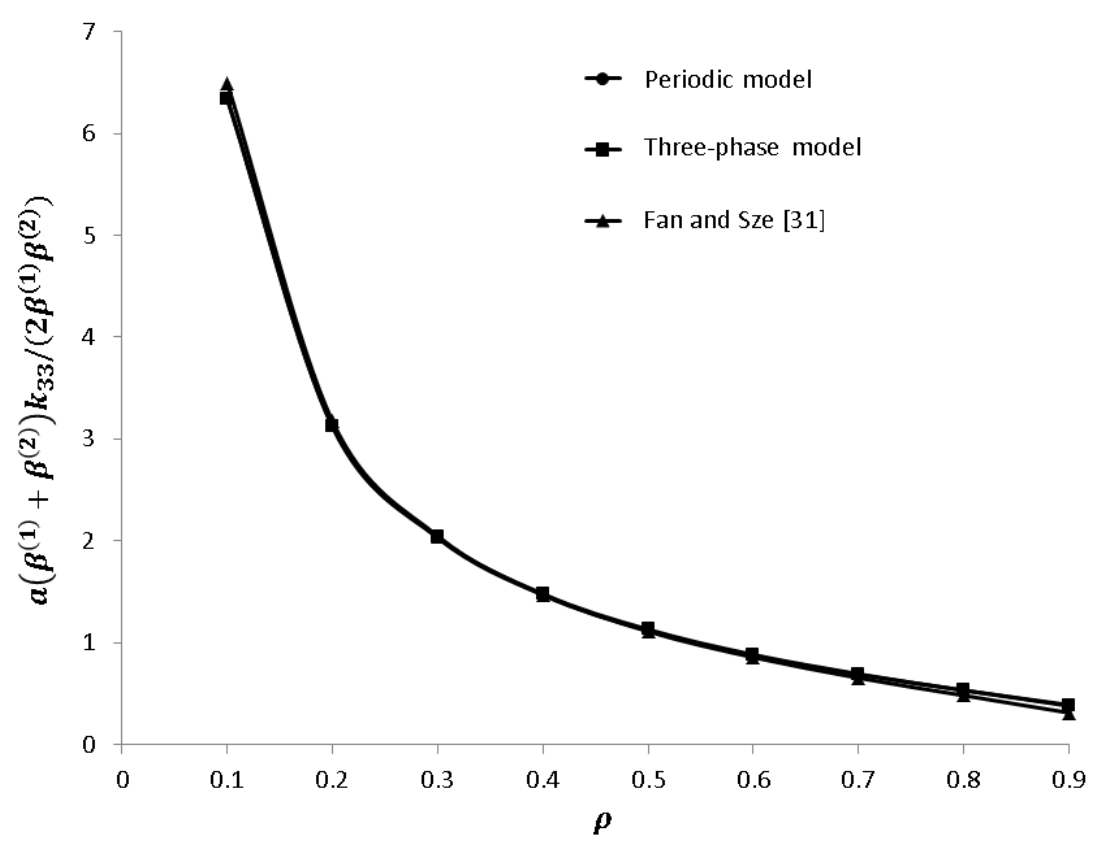

Figure 3.4: Plots of $a\left(\beta^{(1)}+\beta^{(2)}\right) k_{33} /\left(2 \beta^{(1)} \beta^{(2)}\right)$ against $\rho$.

In Figure 3.4, $a\left(\beta^{(1)}+\beta^{(2)}\right) k_{33} /\left(2 \beta^{(1)} \beta^{(2)}\right)$ computed using the finite element method based three-phase model in Fan and Sze [31] is also plotted against $\rho$. It may be of interest to note that as many as ten thousand elements are employed in the finite element calculation for the three-phase model in [31], whereas only 30 collocation points for the representative micro-crack and 40 elements for the effective region are needed in solving the hypersingular integrodifferential equations here for the three-phase model. The plots of $a\left(\beta^{(1)}+\right.$ $\left.\beta^{(2)}\right) k_{33} /\left(2 \beta^{(1)} \beta^{(2)}\right)$ calculated using the two models here and that of [31] are nearly visually indistinguishable for smaller $\rho$, but the percentage difference between the value calculated using the periodic model in Subsection 3.5.1 and that given in [31] ranges from $2.5 \%(\rho=0.10)$ to $25 \%(\rho=0.90)$. The percentage differences in the values of $a\left(\beta^{(1)}+\beta^{(2)}\right) k_{33} /\left(2 \beta^{(1)} \beta^{(2)}\right)$ computed using the periodic model and the three-phase model presented in this paper 
are roughly ten times smaller in magnitude, ranging from around $0.25 \%$ to $2.5 \%$ (as mentioned above). It is obvious that the periodic model is in better agreement with the three-phase model here than with the one in [31]. This is because the three-phase model in [31] ignores the effect that the stress induced by the micro-crack has on the effective region, that is, the model imposes the condition $k_{33} \Delta u_{3}\left(x_{1}\right)=\sigma_{32}^{(\text {ext) }}\left(x_{1}, 0^{+}\right)$on the effective region (instead of $k_{33} \Delta u_{3}\left(x_{1}\right)=\sigma_{32}^{(\mathrm{ext})}\left(x_{1}, 0^{+}\right)+\sigma_{32}^{(\mathrm{imp})}\left(x_{1}, 0^{+}\right)$in (3.33) for the three-phase model here). Thus, it is not surprising that, compared to the three-phase model here, the three-phase model in [31] deviates much more significantly from the periodic model if $\rho$ is very close to unity, that is, if there is a stronger interaction between the micro-crack and the effective region in the three-phase models.

\subsubsection{Inplane Deformations}

For inplane deformations, the elastic moduli (in GPa) for graphite-epoxy and E-glass-epoxy, as given in Table 3.1, are used as the material constants for the upper and lower half-spaces respectively. The values of the elastic moduli for the materials given in Table 3.1 are taken from Glodež et al. [39], Guechaichia and Trendafilova [40] and Rubio-Gonzalez and Mason [69].

In Subsection 3.6.1, we find that the antiplane effective stiffness written in non-dimensionalized form as $a\left(\beta^{(1)}+\beta^{(2)}\right) k_{33} /\left(2 \beta^{(1)} \beta^{(2)}\right)$ is independent of the material constants of the half-spaces. For inplane deformations of the orthotropic half-spaces, it is not clear whether or not the inplane effective stiffness coefficients can be written in similar non-dimensionalized forms that are independent of the material constants. The non-dimensionalized forms used here, namely $a k_{11} / C_{66}^{(2)}$ and $a k_{22} / C_{66}^{(2)}$, depend on not only the damage ratio $\rho$ but also the material constants of the two half-spaces.

Figure 3.5 gives the plots of $a k_{11} / C_{66}^{(2)}$ and $a k_{22} / C_{66}^{(2)}$ against $\rho$. As ex- 
(a)

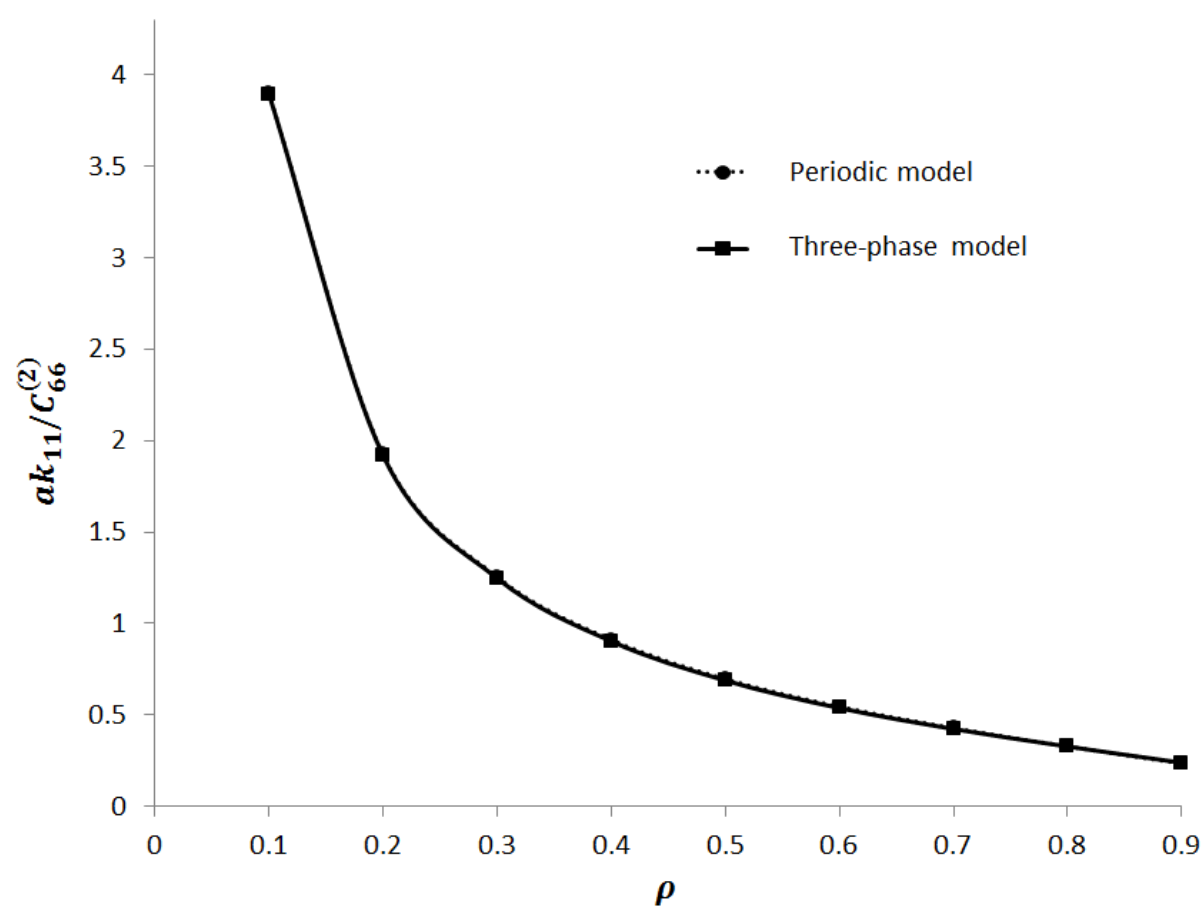

(b)

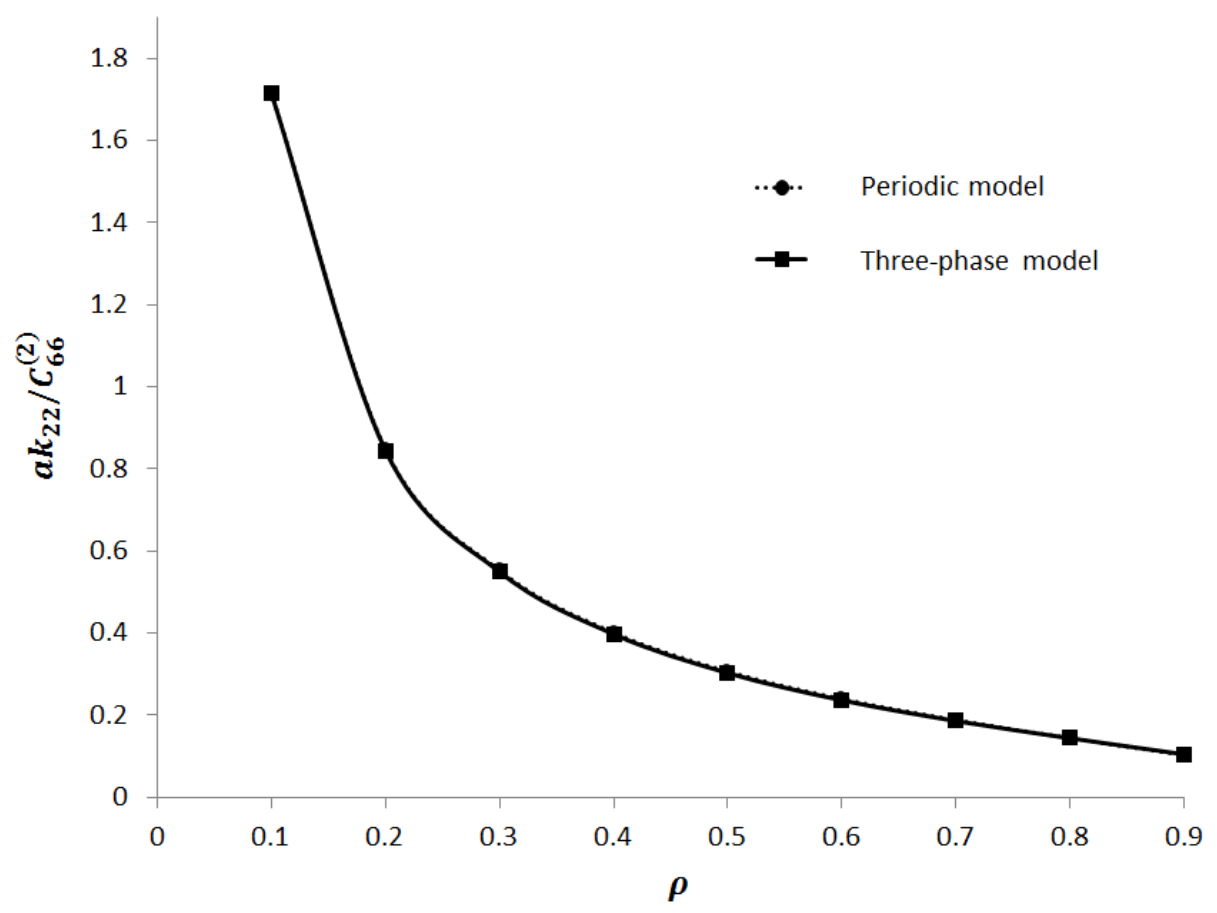

Figure 3.5: Plots of $a k_{11} / C_{66}^{(2)}$ and $a k_{22} / C_{66}^{(2)}$ against $\rho$. 
Table 3.1: Elastic moduli (in GPa) for selected orthotropic materials.

\begin{tabular}{lllll}
\hline & $C_{11}$ & $C_{22}$ & $C_{12}$ & $C_{66}$ \\
\hline Boron-epoxy & 209.1 & 20.0 & 5.1 & 6.4 \\
Carbon-epoxy & 136.2 & 9.2 & 3.9 & 4.3 \\
E-glass-epoxy & 46.1 & 12.6 & 2.9 & 5.5 \\
Graphite-epoxy & 155.4 & 16.3 & 3.7 & 7.5 \\
Martensite & 233.0 & 233.0 & 135.0 & 118.0 \\
\hline
\end{tabular}

pected, the non-dimensionalized effective stiffness coefficients calculated using the periodic model in Subsection 3.5.1 agree well with the corresponding values predicted by the three-phase model in Subsection 3.5.2. The percentage difference of the non-dimensionalized effective stiffness coefficients between the two models is found to range from $0.086 \%$ to $2.1 \%$ for $0.1 \leq \rho \leq 0.9$, with the percentage difference becoming larger as the damage ratio $\rho$ increases.

From Figures 3.4 and 3.5, each of the non-dimensionalized effective stiffness coefficients is observed to decrease in magnitude as the damage ratio $\rho$ increases. This is because the coplanar micro-cracks are closer to one another and are less stable having larger displacement jumps if the damage ratio $\rho$ is larger.

\subsection{Micromechanical-statistical Simulations}

The micromechanical-statistical model is employed here to examine the effective stiffness coefficients of the micro-cracked interface under antiplane and inplane deformations. For the statistical simulation, $N$ interfaces are randomly generated. Each interface is formed by placing randomly $M$ micro-cracks of randomly generated lengths on a period length of the interface. The microcrack length follows a chosen $\chi^{2}$ distribution.

For the $n$-th interface, if the average half crack length is denoted by $\widehat{a}^{(n)}$ 
and the effective stiffness coefficients are denoted by $k_{11}^{(n)}, k_{22}^{(n)}$ (for inplane deformations) and $k_{33}^{(n)}$ (for antiplane deformations), then we non-dimensionalize $k_{11}^{(n)}, k_{22}^{(n)}$ and $k_{33}^{(n)}$ in a similar manner as in Section 3.6, that is, we define the non-dimensionalized effective stiffness coefficients

$$
\begin{aligned}
& K_{11}^{(n)}=\widehat{a}^{(n)} k_{11}^{(n)} / C_{66}^{(2)}, \\
& K_{22}^{(n)}=\widehat{a}^{(n)} k_{22}^{(n)} / C_{66}^{(2)}, \\
& K_{33}^{(n)}=\widehat{a}^{(n)}\left(\beta^{(1)}+\beta^{(2)}\right) k_{33}^{(n)} /\left(2 \beta^{(1)} \beta^{(2)}\right) .
\end{aligned}
$$

The mean values $\widehat{K}_{11}, \widehat{K}_{22}$ and $\widehat{K}_{33}$ of the non-dimensionalized effective stiffness coefficients of the $N$ randomly generated interfaces are calculated by using (3.46) and (3.47).

\subsubsection{Number of Micro-cracks for Homogenizing the In- terface}

To investigate the number of micro-cracks required to homogenize the effective stiffness coefficients of the interface in Figure 3.1, 50 interfaces are randomly generated as described in Subsection 3.4.3. The $\chi^{2}(5)$ distribution is used to generate the lengths of the $M$ micro-cracks on each interface. For inplane deformations, the upper and lower half-spaces are respectively occupied by graphite-epoxy and E-glass-epoxy. If the number of micro-cracks needed to homogenize the interface is $M_{0}$, then the mean values $\widehat{K}_{11}, \widehat{K}_{22}$ (for inplane deformations) and $\widehat{K}_{33}$ (for antiplane deformations) of the non-dimensionalized effective stiffness coefficients of the 50 interfaces are expected to not vary significantly with $M$ for $M \geq M_{0}$.

For $\rho=0.5$, Figure 3.6 gives the scatter plots of the data for the nondimensionalized effective stiffness coefficients of the 50 interfaces against $M$. For a fixed value of $M$, Figure 3.6 also includes the means $\widehat{K}_{11}, \widehat{K}_{22}$ and $\widehat{K}_{33}$ 
(a)

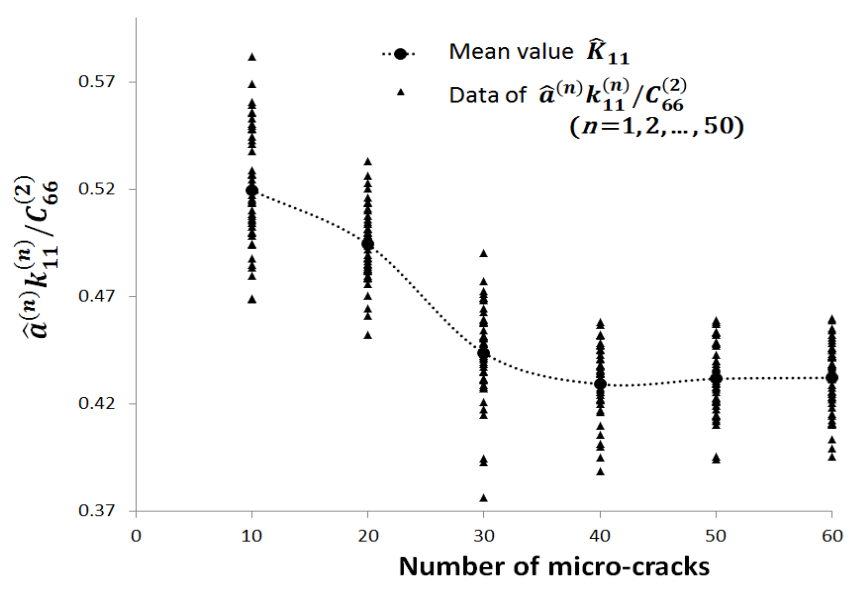

(b)

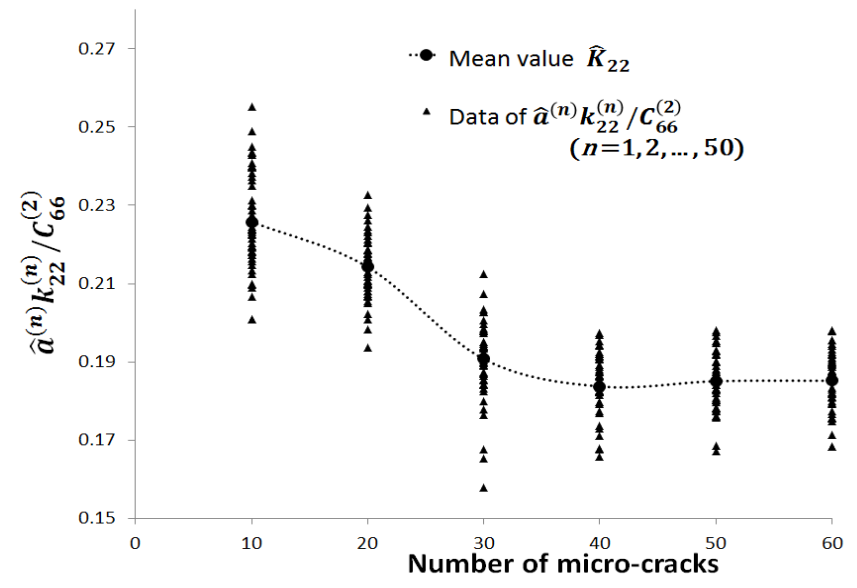

(c)

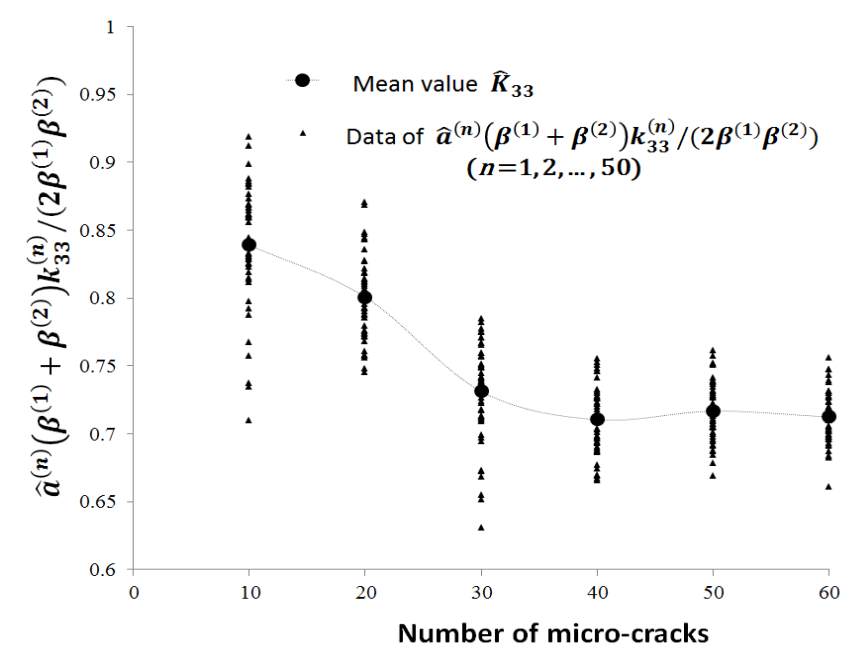

Figure 3.6: Scatter plots and mean values of the non-dimensionalized effective stiffness coefficients against $M$. 
of the non-dimensionalized effective stiffness coefficients of the 50 interfaces.

It is observed that the means $\widehat{K}_{11}, \widehat{K}_{22}$ and $\widehat{K}_{33}$ decrease dramatically as $M$ increases from 10 to 40 and they do not change significantly for $M$ exceeding 40. Furthermore, the ranges of the data of the effective stiffness coefficients of the 50 interfaces for $M=40$ are narrower than those for $M=10$. The ranges of the data do not seem to change very much for $M$ between 40 and 60. It appears that around 40 micro-cracks per period length of the interface may be sufficient to homogenize the interface.

Further investigations show that the number of micro-cracks required for homogenizing the interface may be lower if a $\chi^{2}$ distribution of a higher degree of freedom, such as 10 and 25, is used to generate the lengths of the microcracks.

\subsubsection{Effect of the Micro-crack Length Distribution on the Effective Stiffness Coefficients}

The effects of the micro-crack lengths on the mean values of the effective stiffness coefficients of the interface are examined below.

For a given damage ratio $\rho, N$ interfaces are randomly generated. To generate each interface, the lengths of $M$ micro-cracks are randomly generated using the $\chi^{2}(k)$ distribution where $k$ is a fixed positive integer. The microcracks are then positioned in such a way that the normalized crack-tip gap length $g /(2 \widehat{a})$ between any two adjacent micro-cracks is the same given by

$$
\frac{g}{2 \widehat{a}}=\frac{1-\rho}{\rho},
$$

where $g$ is the crack-tip gap length before normalization and $\widehat{a}$ is the average half length of the $M$ micro-cracks. For a fixed value of $\rho$, the normalized cracktip gap length $g /(2 \widehat{a})$ remains the same for all interfaces within the sample, 
(a)

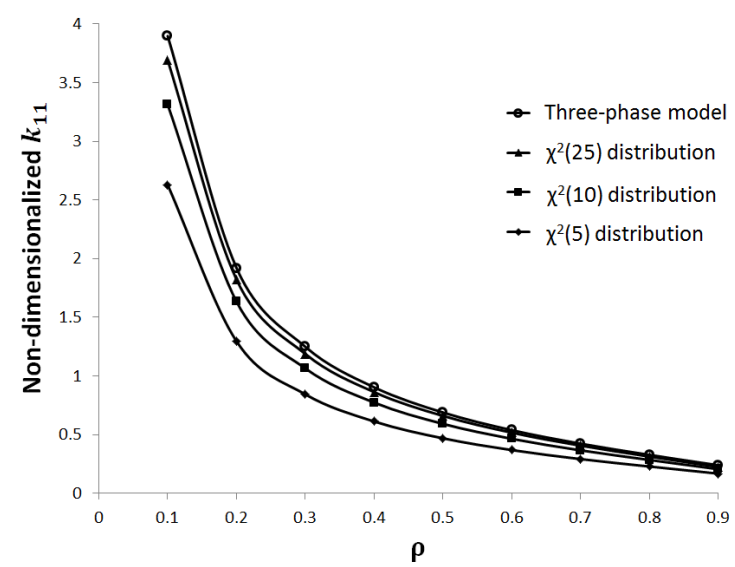

(b)

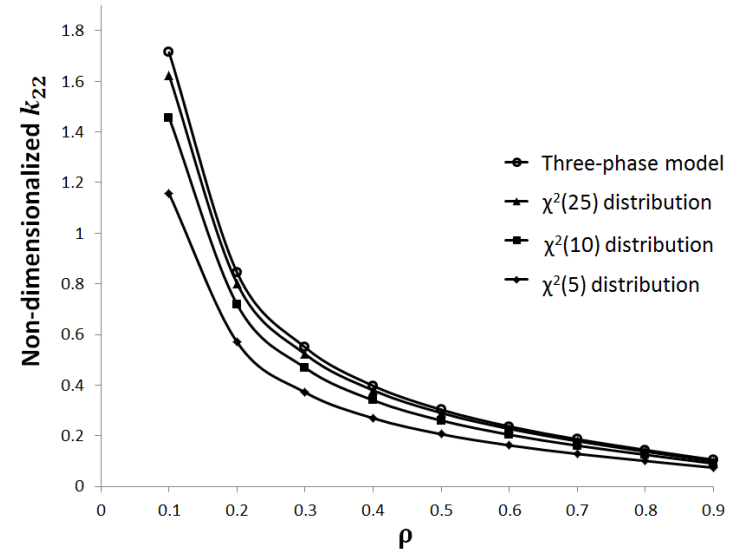

(c)

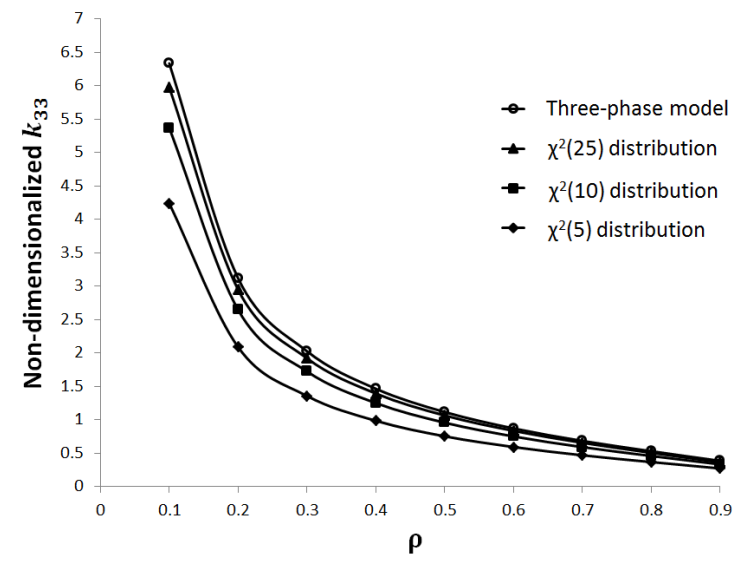

Figure 3.7: Plots of mean values $\widehat{K}_{11}, \widehat{K}_{22}$ and $\widehat{K}_{33}$ against the damage ratio $\rho$, for micro-crack length generated by the $\chi^{2}$ distribution of degrees of freedom 5, 10 and 25. Values predicted by the three-phase model are also plotted. 
but the set of lengths of the micro-cracks is different from one interface to another. As in the inplane simulations in Subsection 3.7.1, the materials in the upper and lower half-spaces for inplane deformations here are selected to be graphite-epoxy and E-glass-expoxy respectively with the elastic moduli given in Table 3.1.

For $\rho=0.5$, the mean values $\widehat{K}_{11}, \widehat{K}_{22}$ (for inplane deformations) and $\widehat{K}_{33}$ (for antiplane deformations) from the 50 interfaces $(N=50)$ with 40 microcracks per period length of each interface $(M=40)$ are plotted in Figure 3.7 against the damage ratio $\rho$ for cases where the lengths of the micro-cracks are generated using $\chi^{2}$ distributions with degrees of freedom 5, 10 and 25 . For comparison purpose, the corresponding values of the non-dimensionalized effective stiffness coefficients calculated using the three-phase model are also plotted against $\rho$ in Figure 3.7.

From Figure 3.7, for a fixed value of $\rho$, the means $\widehat{K}_{11}, \widehat{K}_{22}$ and $\widehat{K}_{33}$ are closer to the corresponding non-dimensionalized effective stiffness coefficients calculated using the three-phase model as the degree of freedom of the $\chi^{2}$ distribution used becomes larger, that is, as the distribution of the microcrack length becomes more normal like. This is as expected, as the threephase model assumes that the micro-cracks are of equal length and are evenly distributed on the interface. Also, for a $\chi^{2}$ distribution of a lower degree of freedom, the mean of each non-dimensionalized stiffness coefficient appears to be smaller. Perhaps this observation on the mean may be explained by taking into consideration that most of the micro-cracks tend to be short and the ratio of the length of the longest micro-crack to that of the shortest micro-crack is relatively large if the $\chi^{2}$ distribution used is of a lower degree of freedom. For example, the ratio is likely around 1000 for the $\chi^{2}(5)$ distribution, but it is likely around 3 for the $\chi^{2}(25)$ distribution. 
Table 3.2: The mean $\widehat{K}_{33}$ and standard deviation $s_{33}$ of the antiplane nondimensionalized effective stiffness coefficient.

\begin{tabular}{cccccccc}
\hline$\rho$ & \multicolumn{2}{c}{$\chi^{2}(5)$} & \multicolumn{2}{c}{$\chi^{2}(10)$} & \multicolumn{2}{c}{$\chi^{2}(25)$} & Three-phase \\
\cline { 2 - 6 } & $\widehat{K}_{33}$ & $s_{33}$ & $\widehat{K}_{33}$ & $s_{33}$ & $\widehat{K}_{33}$ & $s_{33}$ & model \\
\hline 0.1 & 6.209 & 0.015 & 6.327 & 0.001 & 6.336 & 0.0004 & 6.335 \\
\hline 0.2 & 3.009 & 0.013 & 3.110 & 0.001 & 3.124 & 0.001 & 3.120 \\
\hline 0.3 & 1.933 & 0.010 & 2.019 & 0.002 & 2.034 & 0.001 & 2.029 \\
\hline 0.4 & 1.382 & 0.008 & 1.459 & 0.002 & 1.475 & 0.001 & 1.467 \\
\hline 0.5 & 1.045 & 0.005 & 1.111 & 0.002 & 1.125 & 0.001 & 1.118 \\
\hline 0.6 & 0.813 & 0.004 & 0.867 & 0.002 & 0.880 & 0.001 & 0.873 \\
\hline 0.7 & 0.637 & 0.005 & 0.679 & 0.001 & 0.690 & 0.001 & 0.686 \\
\hline 0.8 & 0.490 & 0.003 & 0.522 & 0.001 & 0.531 & 0.001 & 0.532 \\
\hline 0.9 & 0.353 & 0.002 & 0.373 & 0.001 & 0.379 & 0.001 & 0.388 \\
\hline
\end{tabular}

\subsubsection{Effect of the Micro-crack Tip Gap Distribution on the Effective Stiffness Coefficients}

The effects of micro-crack tip gap distribution on the effective stiffness coefficients of the interface are examined below. Note that the micro-crack tip gaps are the perfectly bonded areas given in Figure 3.1.

For a fixed damage ratio $\rho, N$ interfaces are randomly generated by placing $M$ equal length micro-cracks over a period length $L$ of the interface. The micro-crack tip gap $g$ between any two consecutive neighboring micro-cracks is generated randomly using the $\chi^{2}(k)$ distribution. The mean of $g$, denoted by $\bar{g}$, is related to micro-crack half length $a$ by

$$
\frac{2 a}{\bar{g}}=\frac{\rho}{1-\rho} .
$$

For antiplane deformations, the mean $\widehat{K}_{33}$ and standard deviation $s_{33}$ of the non-dimensionalized effective stiffness coefficient calculated using $N=50$ and $M=40$ are shown in Table 3.2 for selected values of the damage ratio 


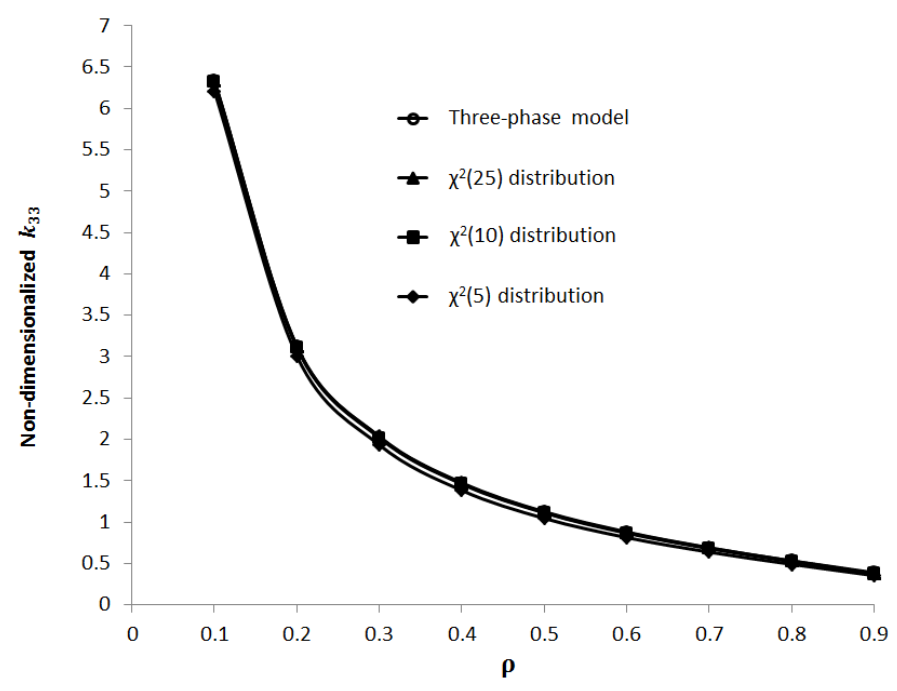

Figure 3.8: Plots of the mean values $\widehat{K}_{33}$ against the damage ratio $\rho$, for distributions of micro-crack tip gap generated using the $\chi^{2}$ distribution of degrees of freedom 5, 10 and 25. Also included are the corresponding values predicted by the three-phase model.

$\rho$ and for micro-crack tip gaps generated using $\chi^{2}$ distributions with degrees of freedom 5, 10 and 25. The corresponding values of the non-dimensionalized effective stiffness coefficient $a\left(\beta^{(1)}+\beta^{(2)}\right) k_{33} /\left(2 \beta^{(1)} \beta^{(2)}\right)$ calculated using the three-phase model are also given in Table 3.2. It is obvious that the mean in Table 3.2 for a fixed value of $\rho$ is smaller if the $\chi^{2}$ distribution used is of a lower degree of freedom. This may be explained by taking into consideration that the $\chi^{2}$ distribution of a lower degree of freedom generates micro-crack tip gaps that skew towards having shorter lengths, giving rise to $\Delta u_{3}$ of a higher magnitude on most of the micro-cracks.

Nevertheless, the graphs of $\widehat{K}_{33}$ against the damage ratio $\rho$ do not show much difference in Figure 3.8 for the $\chi^{2}(5), \chi^{2}(10)$ and $\chi^{2}(25)$ distributions compared to the graphs in Figure 3.7. It seems that varying the distribution of the micro-crack tip gaps does not affect the mean effective stiffness coefficient 
as much as varying the distribution of the lengths of the micro-cracks. This observation is consistent with our understanding that lengths of the microcracks directly affect the displacement jumps over the micro-cracks, while the gap between micro-cracks does not affect the displacement jump as significantly as crack-lengths. Note that the effective stiffness coefficient of the interface is calculated by averaging the displacement jumps.

The statistical simulations for inplane deformations also show that variation of the micro-crack lengths has a greater influence on the non-dimensionalized

effective stiffness coefficients $\widehat{K}_{11}$ and $\widehat{K}_{22}$ than the variation of the micro-crack tip gaps for $0.1 \leq \rho \leq 0.9$ and various combinations of the material constants for the two half-spaces.

\subsubsection{Case Studies Using Specific Orthotropic Materials for Inplane Deformations}

For inplane deformations, the micromechanical-statistical model is used here to simulate the micro-cracked interface between a selected pair of dissimilar orthotropic materials.

As before, for selected values of $\rho, 50$ micro-cracked interfaces are randomly generated for the statistical simulations. For each interface, 40 micro-cracks are randomly chosen with their lengths following the $\chi^{2}(5)$ distribution. The micro-cracks are positioned randomly over a period interval of the interface.

The elastic moduli (in GPa) for boron-epoxy as given in Table 3.1 are used as the material constants for the lower half-space $x_{2}<0$. Three different orthotropic materials of differing strength, namely carbon-epoxy, graphite-epoxy and martensite, with elastic moduli as given in Table 3.1, are selected in turn to be the material in the upper half-space $x_{2}>0$.

For $\rho=0.5$, the means $\widehat{K}_{11}$ and $\widehat{K}_{22}$ against $\rho$ are plotted in Figure 3.9 for 
(a)

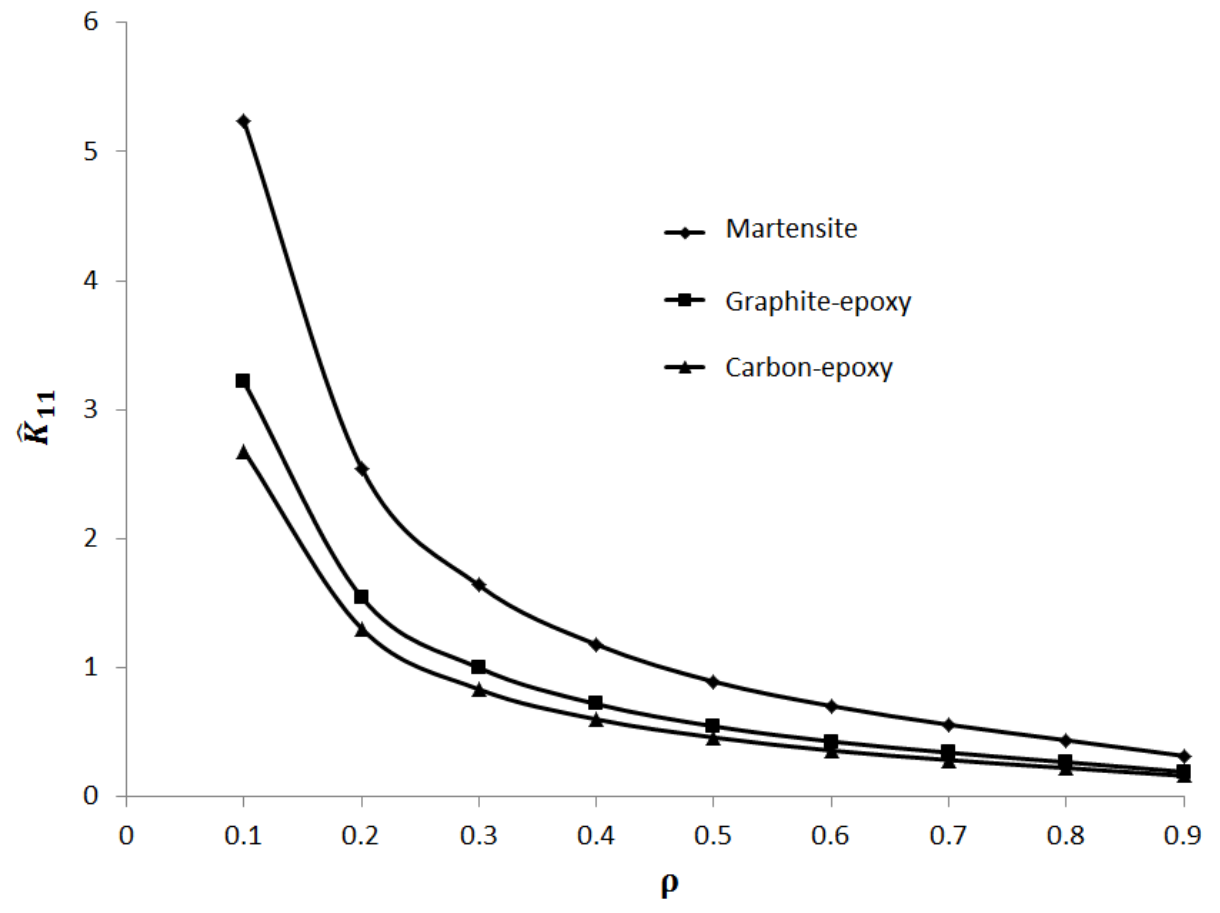

(b)

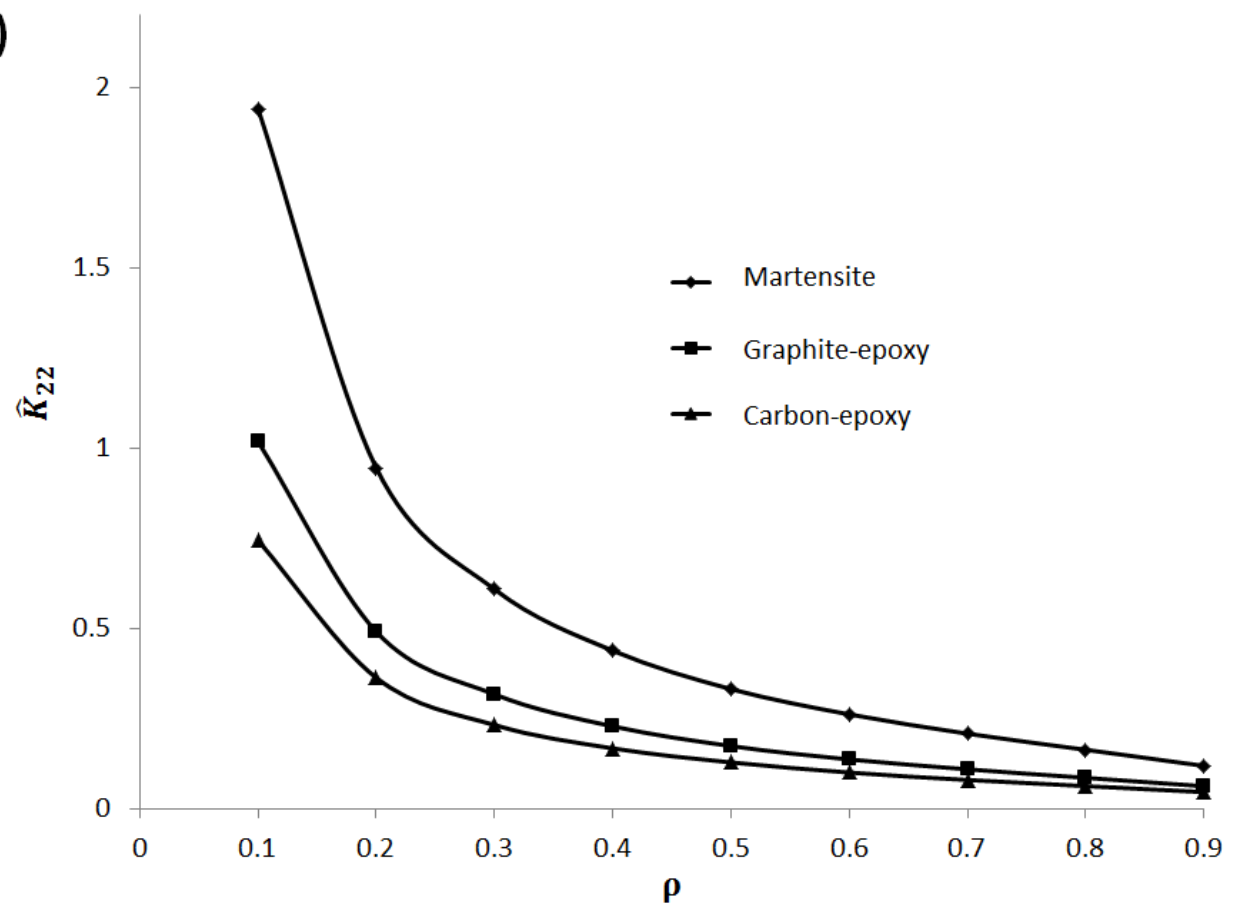

Figure 3.9: Plots of $\widehat{K}_{11}$ and $\widehat{K}_{22}$ against $\rho$ for selected materials in the upper half-space. 
the cases where carbon-epoxy, graphite-epoxy and martensite are used as the material in the upper half-space. It is observed that the interface for the case where the upper half-space is occupied by martensite has the largest effective stiffness coefficients $\widehat{K}_{11}$ and $\widehat{K}_{22}$, while the values of $\widehat{K}_{11}$ and $\widehat{K}_{22}$ are the smallest for the case where the upper half-space is carbon-epoxy. Thus, the effective stiffness coefficients of the interface appear to be larger in magnitude if the material of the upper half-space has larger elastic moduli. This observation is consistent with our intuition that the upper half-space of a "stronger" material jointed on the lower half-space may reduce the magnitudes of the displacement jumps over the micro-cracks to give rise to larger effective stiffness coefficients.

\subsubsection{Isotropic Bimaterial Subject to Inplane Deforma- tions}

The cases where the two elastic half-spaces are both isotropic are considered here for inplane deformations. For isotropic materials, the elastic moduli are given by

$$
\begin{aligned}
& c_{1111}^{(p)}=c_{2222}^{(p)}=\lambda^{(p)}+2 \mu^{(p)}, \\
& c_{1212}^{(p)}=c_{2112}^{(p)}=c_{1221}^{(p)}=c_{2121}^{(p)}=\mu^{(p)}, \\
& c_{1122}^{(p)}=c_{2211}^{(p)}=\lambda^{(p)},
\end{aligned}
$$

where $\lambda^{(p)}$ and $\mu^{(p)}$ are the Lamé constants of the isotropic materials.

The analysis given in Chapter 2 for general orthotropic materials appears

to break down for isotropic materials, as constants such as $N_{\alpha k}^{(p)}$ and $M_{\alpha k}^{(p)}$ are ill-defined if the elastic moduli are given by (3.78). Nevertheless, it may be recovered for isotropic materials by using a limiting procedure given in Ang [9]. More specifically, the hypersingular integral and integro-differential equations 
for the micromechanical-statistical model can be recovered for isotropic materials by replacing $c_{1122}^{(p)}=c_{2211}^{(p)}=\lambda^{(p)}$ in (3.78) with $c_{1122}^{(p)}=c_{2211}^{(p)}=\lambda^{(p)}(1-\varepsilon)$ and letting the real parameter $\varepsilon$ tend to zero.

For isotropic materials, the hypersingular integro-differential equations in (3.30) reduce to

$$
\begin{aligned}
& \frac{1}{2 \pi}\left[\int_{a^{(k)}}^{b^{(k)}} \frac{\Delta u_{r}^{(\mathrm{imp})}\left(x_{1}\right) \widetilde{W}_{j r}}{\gamma\left(x_{1}-\xi_{1}\right)^{2}} d x_{1}\right. \\
& +\sum_{\substack{n=1 \\
n \neq k}}^{M} \int_{a^{(n)}}^{b^{(n)}} \frac{\Delta u_{r}^{(\mathrm{imp})}\left(x_{1}\right) \widetilde{W}_{j r}}{\gamma\left(x_{1}-\xi_{1}\right)^{2}} d x_{1} \\
& \left.+\sum_{\substack{n=1 \\
M}}^{b_{a^{(n)}}^{b^{(n)}}} \frac{1}{\gamma} \Delta u_{r}^{(\mathrm{imp})}\left(x_{1}\right) \widetilde{W}_{j r} \Theta\left(x_{1}, \xi_{1}\right) d x_{1}-\frac{\pi \widetilde{V}_{j r}}{\gamma} \frac{d\left(\Delta u_{r}^{(\mathrm{imp})}\left(\xi_{1}\right)\right)}{d \xi_{1}}\right] \\
= & \sigma_{j 2}^{(\mathrm{ext})}\left(\xi_{1}, 0^{+}\right) \text {for } a^{(k)}<\xi_{1}<b^{(k)}(k=1,2, \ldots, M),
\end{aligned}
$$

where $\gamma$ is defined as

$$
\gamma=\frac{\lambda^{(1)}+2 \mu^{(1)}}{\mu^{(1)}\left(\lambda^{(1)}+\mu^{(1)}\right)}+\frac{\lambda^{(2)}+2 \mu^{(2)}}{\mu^{(2)}\left(\lambda^{(2)}+\mu^{(2)}\right)},
$$

and $\widetilde{W}_{r i}$ and $\widetilde{V}_{r i}$ are real constants given by

$$
\begin{gathered}
{\left[\widetilde{W}_{r i}\right]=\left[\begin{array}{cc}
-\frac{4}{1-\beta^{2}} & 0 \\
0 & -\frac{4}{1-\beta^{2}}
\end{array}\right],} \\
{\left[\widetilde{V}_{r i}\right]=\left[\begin{array}{cc}
0 & \frac{4 \beta}{1-\beta^{2}} \\
-\frac{4 \beta}{1-\beta^{2}} & 0
\end{array}\right],}
\end{gathered}
$$

with $\beta$ being one of the Dundurs' parameters defined in Schmauder [71] and Schmauder and Meyer [72] as

$$
\beta=\frac{1}{\gamma}\left(\frac{1}{\lambda^{(1)}+\mu^{(1)}}-\frac{1}{\lambda^{(2)}+\mu^{(2)}}\right) .
$$




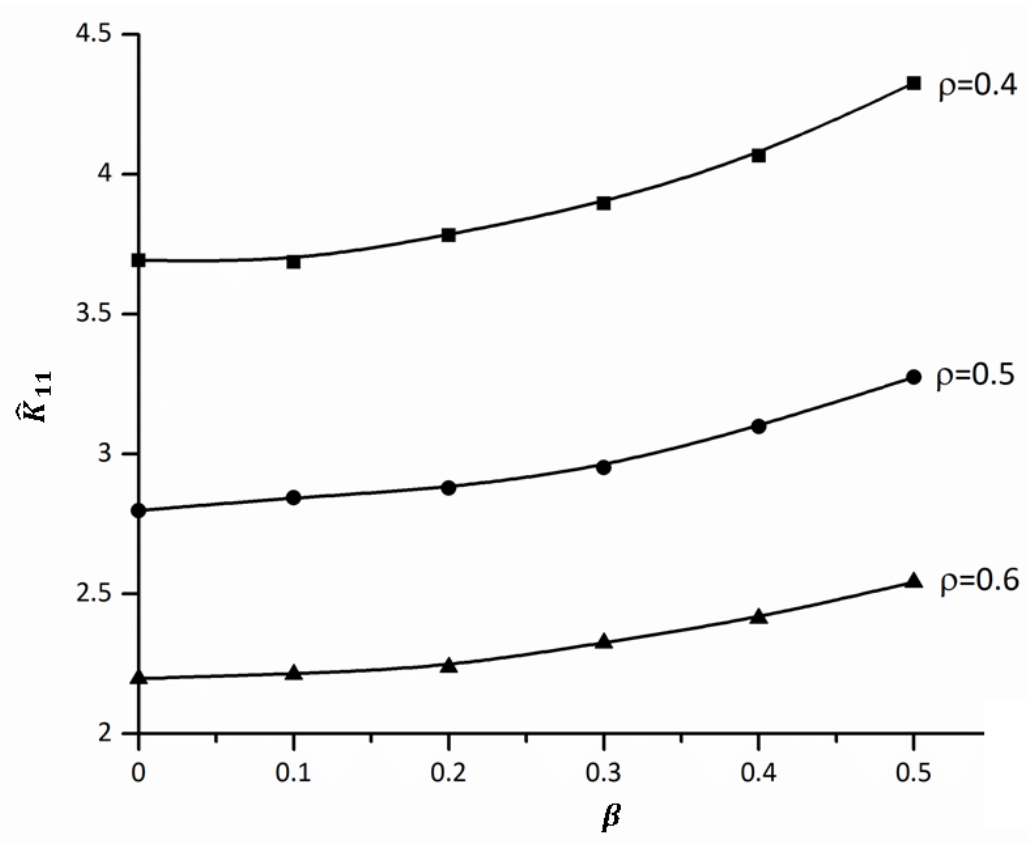

Figure 3.10: Plots of $\widehat{K}_{11}$ against $\beta$ for selected values of $\rho$.

To perform micromechanical-statistical simulations for estimating the nondimensionalized inplane effective stiffness coefficients of the interface for given values of $\beta$ and $\rho, 50$ micro-cracked interfaces are randomly generated. For each interface, the $\chi^{2}(5)$ distribution is used to randomly generate the 40 micro-cracks which are then randomly located over a period interval of the interface. For the $n$-th interface between two isotropic half-spaces, the nondimensionalized inplane effective stiffness coefficients $K_{11}^{(n)}$ and $K_{22}^{(n)}$ in (3.77) are respectively defined by $K_{11}^{(n)}=\gamma \widehat{a}^{(n)} k_{11}^{(n)}$ and $K_{22}^{(n)}=\gamma \widehat{a}^{(n)} k_{22}^{(n)}$. The means $\widehat{K}_{11}$ and $\widehat{K}_{22}$ of the non-dimensionalized effective stiffness coefficients of the 50 interfaces are calculated by using (3.77).

For a given interface between two isotropic half-spaces, $\widehat{K}_{11}$ and $\widehat{K}_{22}$ vary with only the Dundurs' parameter $\beta$. This is obvious from the hypersingular integro-differential equations (3.79), if we note that $\widetilde{W}_{r i}$ and $\widetilde{V}_{r i}$ depend on $\beta$ 
only. Also, note that $\widehat{K}_{11}=\widehat{K}_{22}$ here. The value of $\widehat{K}_{11}$ computed from the sample of 50 randomly generated micro-cracked interfaces are plotted against $\beta$ for $\beta \geq 0$ in Figure 3.10 for selected values of $\rho$. For $\beta \geq 0$ and for a fixed $\rho$, the value of $\widehat{K}_{11}$ increases as $\beta$ increases. Note that $\widehat{K}_{11}$ here is an even function of $\beta$, since the effective stiffness coefficients remain the same if the materials in the two half-spaces are interchanged.

\subsection{Summary}

Three different micromechanical models based on the hypersingular integral formulations are proposed here for the task of estimating the effective stiffness coefficients of a micro-cracked interface between two dissimilar elastic half-spaces under antiplane and inplane deformations. The first model, which is called the three-phase model, simplifies a period interval of the interface into three parts - a representative micro-crack, perfectly bonded and effective regions. In the second model, the periodic interfacial micro-cracks are taken to be of equal length and are evenly distributed on the interface, that is, the interface may be simplified to one that contains one micro-crack over a period interval. The third one is the micromechanical-statistical model, in which a period interval of the interface contains a selected number of randomly positioned micro-cracks with statistically varying sizes.

Numerical values of the effective stiffness coefficients computed using the periodic model are compared with the corresponding ones calculated using the three-phase model. As expected, the two sets of values show good agreement for a wide range of the damage ratio $\rho$ of the interface.

Micromechanical simulations of interfaces under antiplane and inplane deformations suggest that around 40 or more micro-cracks per period length 
of the interface are required to homogenize the effective stiffness coefficients. Furthermore, the micromechanical simulations show that for a highly skewed crack-length distribution such as $\chi^{2}(5)$, the mean values of the effective stiffness coefficients by statistical simulations may be $30 \%$ lower than the corresponding ones predicted by the three-phase model. If the micro-crack length is generated using a less skewed $\chi^{2}$ distribution such as $\chi^{2}(25)$, the mean effective stiffness coefficients are found to agree with the corresponding ones given by the threephase model to within $6 \%$. For a fixed damage ratio $\rho$, the distribution of the micro-crack length appears to have a stronger influence on the mean values of the effective stiffness coefficients than the distribution of the micro-crack tip gaps.

The specific case studies conducted using the micromechanical-statistical model for inplane deformations show that for a fixed material in the lower half-space, the effective stiffness coefficients are larger in magnitudes when the orthotropic material in the upper half-space is "stronger".

For inplane deformations, we have shown how the material constants in the hypersingular integro-differential equations for the degenerate case in which the materials in the half-spaces are isotropic can be grouped together in terms of one of the Dundurs' parameters for isotropic bimaterials. For the degenerate case, plots of appropriately non-dimensionalized effective stiffness coefficients against the Dundurs' parameter are obtained. 


\section{Chapter 4}

\section{Weak Interfaces between Thin Orthotropic Layers and Orthotropic Half-spaces}

\subsection{Introduction}

This chapter is concerned with formulating micromechanical models like the ones in Chapter 3 for estimating the effective stiffness of a micro-cracked interface between a thin orthotropic elastic layer and an orthotropic elastic halfspace under antiplane and inplane deformations. The width of the layer is comparable to the lengths of the interfacial micro-cracks.

Due to the presence of the external plane boundary of the layer, the method of solution based on the Fourier integral representations for the displacement and stress fields as used in Chapter 3 cannot be easily extended to solve the boundary value problems for the micromechanical models here. Instead of the Fourier integral approach, the boundary integral equations and the perfect interface Green's functions given in Sections 2.3 and 2.4 are used hypersingular boundary integro-differential equations for the micromechanical models. The unknown functions in the hypersingular boundary integro-differential equa- 
tions are the displacement jumps across the micro-damaged interface between the thin layer and the half-space and the displacements on the external plane boundary of the layer.

Numerical methods for solving the hypersingular boundary integro-differential equations and estimating the effective stiffness of the interface are outlined here. The effects of the external plane boundary of the thin layer, the microcrack density and the material properties of the thin layer and the half-space on the effective stiffness of the interface are investigated.

\subsection{Micromechanical Models}

Consider an infinitely long elastic layer of micro-width $h$ bonded to an elastic half-space. With reference to a Cartesian coordinate frame $O x_{1} x_{2} x_{3}$, the layer occupies the region $0<x_{2}<h$ and the elastic half-space $x_{2}<0$. The geometries of the layer and the half-space do not vary along the $x_{3}$ direction. The materials in the layer and the half-space are orthotropic, having possibly dissimilar properties. The bimaterial undergoes either antiplane or inplane elastostatic deformations governed by the basic equations given in Section 2.2. The material constants for the orthotropic materials here are given by

$$
\begin{aligned}
& \left(C_{11}, C_{22}, C_{12}, C_{66}, C_{44}, C_{55}\right) \\
= & \begin{cases}\left(C_{11}^{(1)}, C_{22}^{(1)}, C_{12}^{(1)}, C_{66}^{(1)}, C_{44}^{(1)}, C_{55}^{(1)}\right) & \text { for } 0<x_{2}<h, \\
\left(C_{11}^{(2)}, C_{22}^{(2)}, C_{12}^{(2)}, C_{66}^{(2)}, C_{44}^{(2)}, C_{55}^{(2)}\right) & \text { for } x_{2}<0 .\end{cases}
\end{aligned}
$$

The interface between the thin layer and the half-space is damaged containing periodic arrays of interfacial micro-cracks. A period interval of the interface contains $M$ arbitrarily located micro-cracks of possibly different lengths. The tips of the micro-cracks on the whole interface are as described in Section 3.2. Refer to Figure 4.1 for a sketch of the micro-cracked interface between the thin 
layer and the half-space.

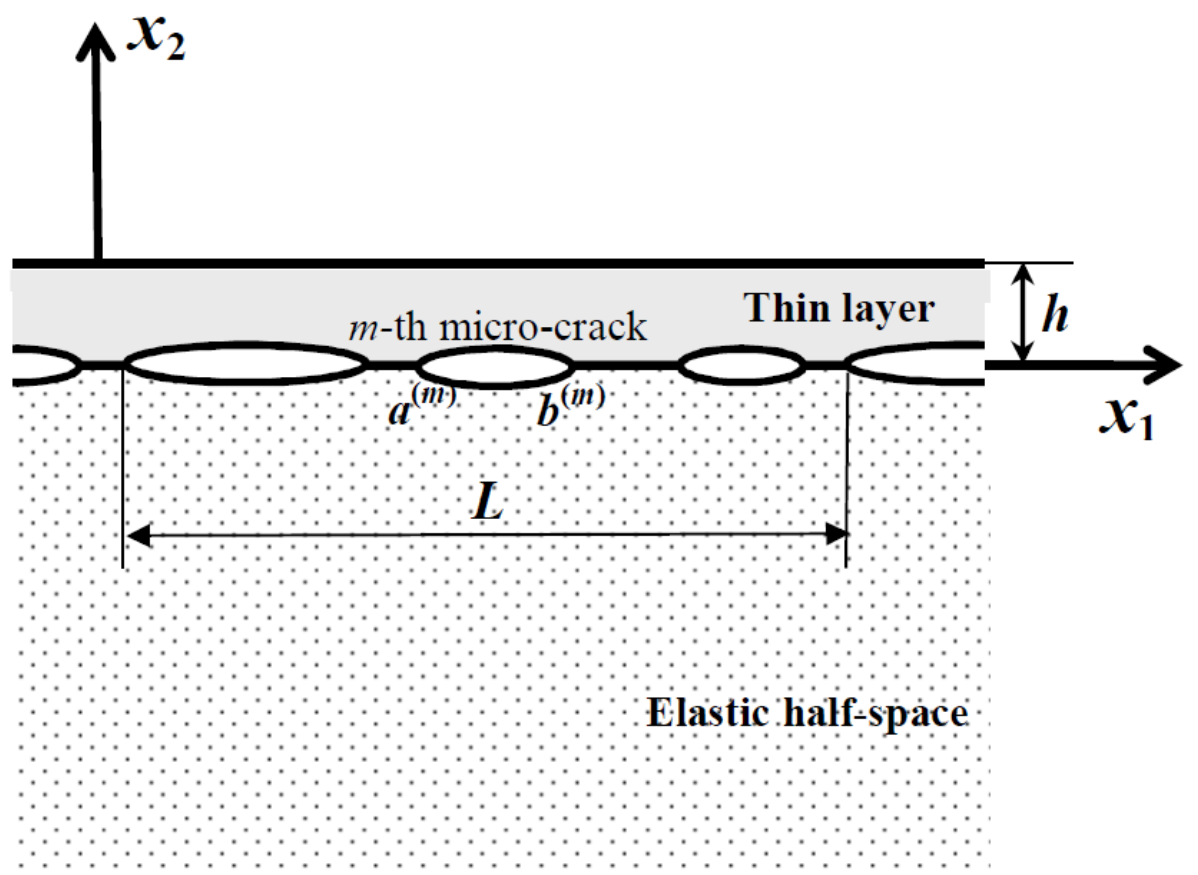

Figure 4.1: A sketch of the micro-cracked interface between the thin orthotropic layer and the orthotropic half-space. Note that $L$ is the period length of the periodically repeated micro-cracked interface and $\left(a^{(m)}, 0\right)$ and $\left(b^{(m)}, 0\right)$ are respectively the left and the right tips of the $m$-th micro-crack.

For a mathematically more amenable analysis of the bimaterial in Figure 4.1, the micro-cracked interface may be replaced by a spring-like interface with interfacial conditions given by (3.3) and (3.4). The effective stiffness coefficients $k_{i j}(i, j=1,2)$ and $k_{33}$ for the micro-cracked interface between the thin orthotropic layer and the orthotropic half-space are needed in the model for the spring-like interface. These coefficients are to be estimated by taking into consideration not only the details of the interfacial micro-cracks and the material properties of the layer and the half-space but also the external plane boundary of the layer. 
Micromechanical models like the ones in Chapter 3 are proposed here for the purpose of estimating the effective stiffness coefficients of the micro-cracked interface. The models are described in the subsections below.

\subsubsection{Three-phase Model}

A sketch of the three-phase model for the micro-cracked interface between the thin orthotropic layer and the orthotropic half-space is given in Figure 4.2. The three-phase model simplifies the period interval $0<x_{1}<L$ of the micro-cracked interface in Figure 4.1 to three distinct parts: (a) a single representative micro-crack, (b) perfectly bonded parts, and (c) an effective region. A detailed description of the interface is given in Subsection 3.4.1.

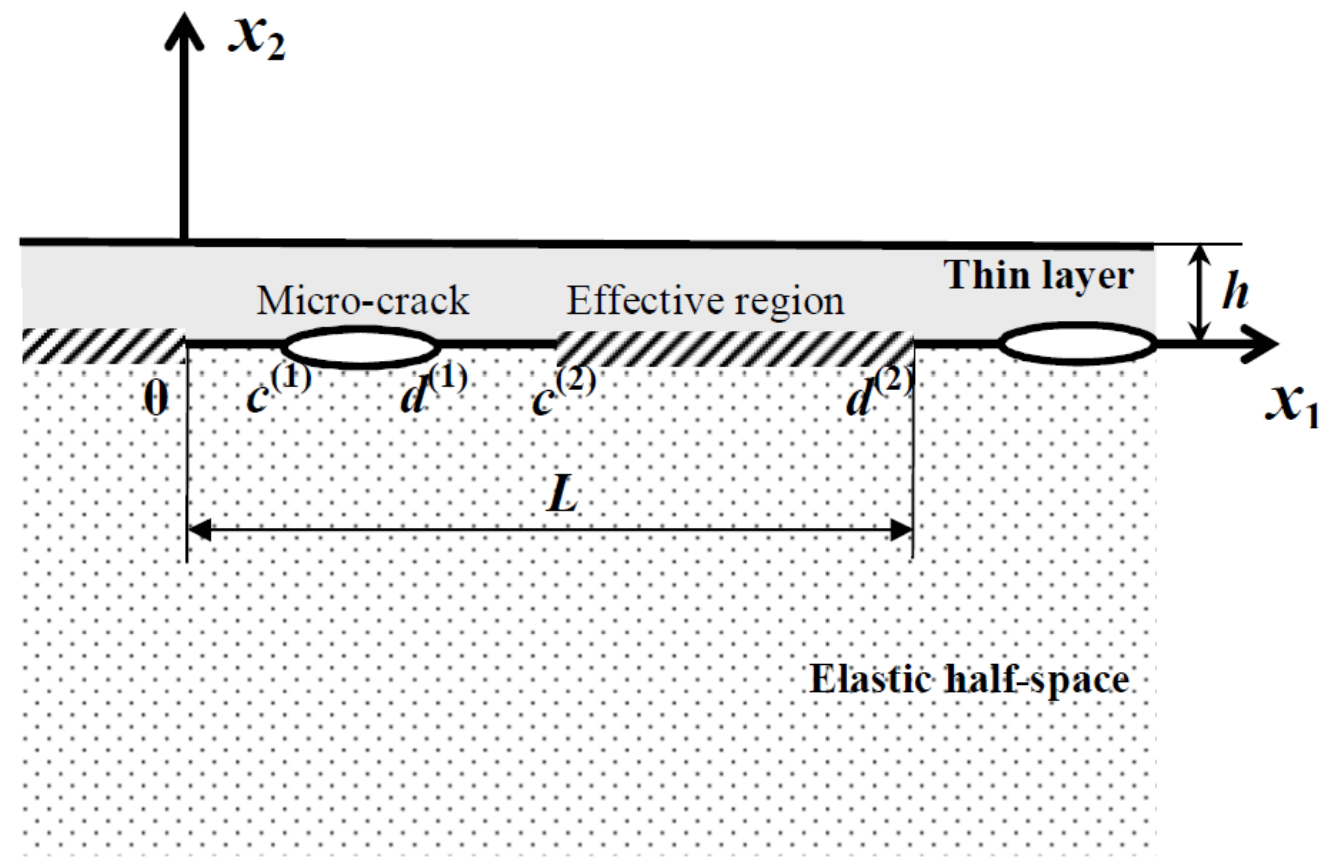

Figure 4.2: A sketch of the three-phase model for the micro-cracked interface between the thin orthotropic layer and the orthotropic half-space. The interface comprises three distinct parts as described in Subsection 3.4.1. 


\subsubsection{Periodic Model Involving Evenly Distributed Micro- cracks of Equal Length}

In this model, the interface between the thin layer and the half-space contains a periodic array of evenly distributed micro-cracks of equal length $2 a$. A sketch of the model is shown in Figure 4.3. Details on the tips of the micro-cracks for the periodic model here are as given in Subsection 3.4.2.

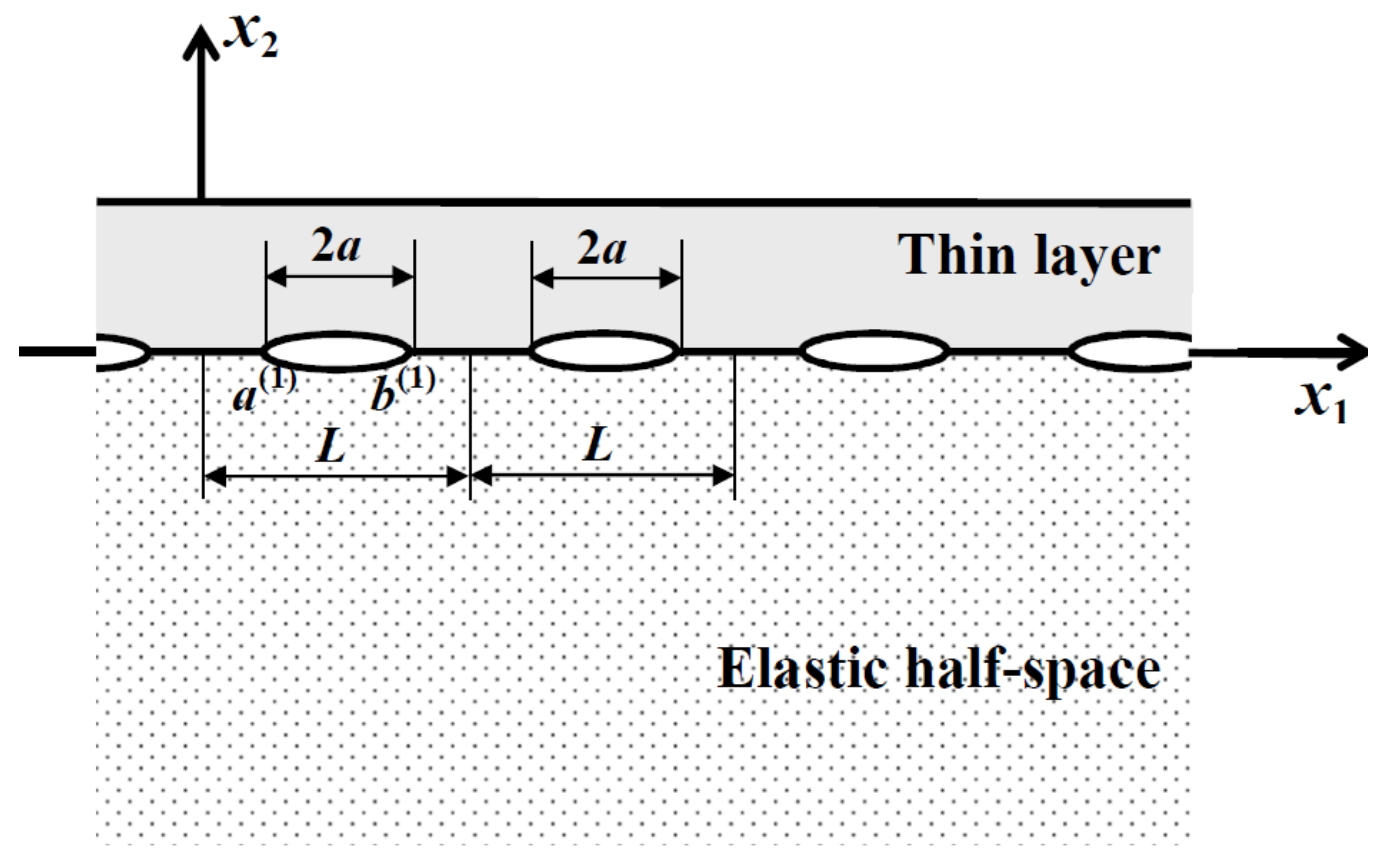

Figure 4.3: A sketch of the periodic model for the micro-cracked interface between the thin orthotropic layer and the orthotropic half-space. The interface contains a periodic array of evenly distributed micro-cracks of equal length $2 a$.

\subsubsection{Micromechanical-statistical Model}

In the micromechanical-statistical model, the micro-cracks over a period interval of the interface between the thin layer and the half-space are generated randomly. 
For fixed values of the width $h$ of the thin layer, the material constants in the orthotropic bimaterial, the average length $\widehat{a}$ of the micro-cracks given in (3.43), the damage ratio $\rho$ given in (3.2) and the number of micro-cracks on a period length of the interface, $N$ interfaces are randomly generated as described in Subsection 3.4.3 to form a statistical sample. More specifically, the lengths of the $M$ micro-cracks for each interface in the sample follow a chosen $\chi^{2}$ distribution of the degree of freedom $m_{0}$, and the $M$ micro-cracks are then randomly positioned on the interval $0<x_{1}<L$. The $N$ randomly generated interfaces in the sample are used to estimate the effective stiffness coefficients.

\subsection{Hypersingular Boundary Integro-differential Equations}

The interfacial micro-cracks in the micromechanical models in Section 4.2 are assumed to be traction free under a suitably prescribed external load on the external plane boundary $x_{2}=h$ of the thin layer and at the far field where $x_{2} \rightarrow-\infty$. The load is assumed to be a periodic function of $x_{1}$ with period $L$.

The displacements and the stresses are written as

$$
\left.\begin{array}{l}
u_{3}=u_{3}^{(\text {ext })}+u_{3}^{(\mathrm{imp})}, \\
\sigma_{3 j}=\sigma_{3 j}^{(\text {(ext) }}+\sigma_{3 j}^{(\text {(imp) }},
\end{array}\right\} \text { for antiplane deformations, }
$$

and

$$
\left.\begin{array}{l}
u_{r}=u_{r}^{(\mathrm{ext})}+u_{r}^{(\mathrm{imp})}, \\
\sigma_{r j}=\sigma_{r j}^{(\mathrm{ext})}+\sigma_{r j}^{(\mathrm{imp})},
\end{array}\right\} \text { for inplane deformations, }
$$

where $u_{3}^{(\text {ext) }}, \sigma_{3 j}^{(\text {ext })}, u_{r}^{(\text {ext })}$ and $\sigma_{r j}^{(\text {ext) }}$ denote the elastic fields for the corresponding cases where the interface is wholly perfect and $u_{3}^{(\mathrm{imp})}, \sigma_{3 j}^{(\mathrm{imp})}, u_{r}^{(\mathrm{imp})}$ and $\sigma_{r j}^{(i m p)}$ are the elastic fields induced by the micro-cracks. Note that all these elastic fields are periodic functions of $x_{1}$ with period $L$. 
The conditions for $\sigma_{32}^{(\mathrm{imp})}$ and $\sigma_{r 2}^{(\mathrm{imp})}$ on the plane boundary $x_{2}=h$ of the layer and at the far field where $x_{2} \rightarrow-\infty$ are given by

$$
\left.\begin{array}{l}
\sigma_{32}^{\text {(imp) }}\left(x_{1}, h\right)=0 \text { for }-\infty<x_{1}<\infty, \\
\sigma_{32}^{\text {(imp) }}\left(x_{1}, x_{2}\right) \rightarrow 0 \text { as } x_{2} \rightarrow-\infty
\end{array}\right\} \text { for antiplane deformations, }
$$

and

$$
\left.\begin{array}{l}
\sigma_{r 2}^{\text {(imp) }}\left(x_{1}, h\right)=0 \text { for }-\infty<x_{1}<\infty, \\
\sigma_{r 2}^{\text {(imp) }}\left(x_{1}, x_{2}\right) \rightarrow 0 \text { as } x_{2} \rightarrow-\infty,
\end{array}\right\} \text { for inplane deformations. }
$$

For each of the micromechanical models in Section 4.2, hypersingular boundary integro-differential equations are derived to express the conditions for the elastic fields $u_{3}^{(\mathrm{imp})}, \sigma_{32}^{(\mathrm{imp})}, u_{r}^{(\mathrm{imp})}$ and $\sigma_{r j}^{(\mathrm{imp})}$ on the interface and on the plane boundary $x_{2}=h$ of the thin layer as follows.

\subsubsection{Three-phase Model}

For the three-phase model under antiplane deformations, the conditions on the interface are given by (3.33). With $u_{3}^{(\text {ext })}$ and $\sigma_{32}^{(\text {ext) }}$ assumed known, the boundary value problem here is to solve (2.5) together with (4.1) subject to the conditions (3.33) on the interface and the conditions in (4.4).

The boundary integral equations (2.20) for antiplane deformations together with the corresponding perfect interface Green's function (2.32) and (2.34) are used to solve the boundary value problem. The antiplane displacement $u_{3}^{(\mathrm{imp})}$ 
is given by

$$
\begin{aligned}
u_{3}^{(\mathrm{imp})}\left(\xi_{1}, \xi_{2}\right)= & \sum_{n=-\infty}^{\infty} \int_{n L}^{(n+1) L} u_{3}^{(\mathrm{imp})}\left(x_{1}, h\right) \\
& \times\left[\frac{1}{2 \pi \beta^{(1)}} \operatorname{Re}\left\{\frac{L_{2}^{(1)}}{x_{1}-\xi_{1}+\tau^{(1)}\left(h-\xi_{2}\right)}\right\}\right. \\
& \left.+\frac{\beta^{(1)}-\beta^{(2)}}{2 \pi \beta^{(1)}\left(\beta^{(1)}+\beta^{(2)}\right)} \operatorname{Re}\left\{\frac{L_{2}^{(1)}}{x_{1}-\xi_{1}+\tau^{(1)} h-\bar{\tau}^{(1)} \xi_{2}}\right\}\right] d x_{1} \\
& -\sum_{n=-\infty}^{\infty} \sum_{m=1}^{2} \int_{c^{(m)}+n L}^{d^{(m)}+n L} \Delta u_{3}^{(\mathrm{imp})}\left(x_{1}\right) \\
& \times\left[\frac{1}{2 \pi \beta^{(1)}} \operatorname{Re}\left\{\frac{L_{2}^{(1)}}{x_{1}-\xi_{1}-\tau^{(1)} \xi_{2}}\right\}\right. \\
& \left.+\frac{\beta^{(1)}-\beta^{(2)}}{2 \pi \beta^{(1)}\left(\beta^{(1)}+\beta^{(2)}\right)} \operatorname{Re}\left\{\frac{L_{2}^{(1)}}{x_{1}-\xi_{1}-\bar{\tau}^{(1)} \xi_{2}}\right\}\right] d x_{1} \quad \text { for } 0<\xi_{2}<h,
\end{aligned}
$$

where $\beta^{(p)}$ and $\tau^{(p)}$ are defined below (2.32) and $L_{2}^{(1)}$ is defined in (2.35).

According to the generalized Hooke's law, (4.6) gives rise to the stress formula

$$
\begin{aligned}
\sigma_{32}^{(\mathrm{imp})}\left(\xi_{1}, \xi_{2}\right)= & \sum_{n=-\infty}^{\infty} \int_{n L}^{(n+1) L} u_{3}^{(\mathrm{imp})}\left(x_{1}, h\right) \\
& \times\left[\frac{1}{2 \pi \beta^{(1)}} \operatorname{Re}\left\{\frac{\left(L_{2}^{(1)}\right)^{2}}{\left[x_{1}-\xi_{1}+\tau^{(1)}\left(h-\xi_{2}\right)\right]^{2}}\right\}\right. \\
& \left.+\frac{\beta^{(1)}-\beta^{(2)}}{2 \pi \beta^{(1)}\left(\beta^{(1)}+\beta^{(2)}\right)} \operatorname{Re}\left\{\frac{L_{2}^{(1)} \bar{L}_{2}^{(1)}}{\left(x_{1}-\xi_{1}+\tau^{(1)} h-\bar{\tau}^{(1)} \xi_{2}\right)^{2}}\right\}\right] d x_{1} \\
& -\sum_{n=-\infty}^{\infty} \sum_{m=1}^{2} \int_{c^{(m)}+n L}^{d^{(m)}+n L} \Delta u_{3}^{(\mathrm{imp})}\left(x_{1}\right) \\
& \times\left[\frac{1}{2 \pi \beta^{(1)}} \operatorname{Re}\left\{\frac{\left(L_{2}^{(1)}\right)^{2}}{\left(x_{1}-\xi_{1}-\tau^{(1)} \xi_{2}\right)^{2}}\right\}\right.
\end{aligned}
$$




$$
\begin{gathered}
\left.+\frac{\beta^{(1)}-\beta^{(2)}}{2 \pi \beta^{(1)}\left(\beta^{(1)}+\beta^{(2)}\right)} \operatorname{Re}\left\{\frac{L_{2}^{(1)} \bar{L}_{2}^{(1)}}{\left(x_{1}-\xi_{1}-\bar{\tau}^{(1)} \xi_{2}\right)^{2}}\right\}\right] d x_{1} \\
\text { for } 0<\xi_{2}<h .
\end{gathered}
$$

Note that no integration is required in (4.6) and (4.7) over the perfectly bonded parts of the interface between the thin layer and the half-space because of the use of the special Green's function for wholly perfect interfaces.

From the summation formula in (2.45) and the stress formula (4.7), the boundary condition on the external plane boundary $x_{2}=h$ as given in (4.4) gives rise to the hypersingular boundary integral equation

$$
\begin{aligned}
& f_{0}^{d^{(2)}} \frac{u_{3}^{(\mathrm{imp})}\left(x_{1}, h\right)}{\left(x_{1}-\xi_{1}\right)^{2}} d x_{1} \\
& +\int_{0}^{d^{(2)}} u_{3}^{(\mathrm{imp})}\left(x_{1}, h\right)\left[\Theta\left(x_{1}, \xi_{1}\right)-\frac{\beta^{(1)}-\beta^{(2)}}{\beta^{(1)}+\beta^{(2)}} \Omega_{0}\left(x_{1},-\xi_{1}, \frac{2 \beta^{(1)}}{C_{44}^{(1)}} h\right)\right] d x_{1} \\
& -\frac{2 \beta^{(2)}}{\beta^{(1)}+\beta^{(2)}} \sum_{k=1}^{2} \int_{c^{(k)}}^{d^{(k)}} \Delta u_{3}^{(\mathrm{imp})}\left(x_{1}\right) \Omega_{0}\left(x_{1},-\xi_{1},-\frac{\beta^{(1)}}{C_{44}^{(1)}} h\right) d x_{1} \\
& =0 \text { for } 0<\xi_{1}<d^{(2)},
\end{aligned}
$$

and the conditions in (3.6) for the micro-cracked parts of the interface yield

$$
\begin{aligned}
& \int_{0}^{d^{(2)}} u_{3}^{(\mathrm{imp})}\left(x_{1}, h\right) \Omega_{0}\left(x_{1},-\xi_{1}, \frac{\beta^{(1)}}{C_{44}^{(1)}} h\right) d x_{1} \\
& -f_{c^{(n)}}^{d^{(n)}} \frac{\Delta u_{3}^{(\mathrm{imp})}\left(x_{1}\right)}{\left(x_{1}-\xi_{1}\right)^{2}} d x_{1}-\sum_{\substack{k=1 \\
k \neq n}}^{2} \int_{c^{(k)}}^{d^{(k)}} \frac{\Delta u_{3}^{(\mathrm{imp})}\left(x_{1}\right)}{\left(x_{1}-\xi_{1}\right)^{2}} d x_{1}
\end{aligned}
$$




$$
\begin{aligned}
& -\sum_{k=1}^{2} \int_{c^{(k)}}^{d^{(k)}} \Delta u_{3}^{(\mathrm{imp})}\left(x_{1}\right) \Theta\left(x_{1}, \xi_{1}\right) d x_{1} \\
= & \frac{\pi\left(\beta^{(1)}+\beta^{(2)}\right)\left(\sigma_{32}^{(\mathrm{ext})}\left(\xi_{1}, 0^{+}\right)-\delta^{(n 2)} k_{33} \Delta u_{3}^{(\mathrm{imp})}\left(\xi_{1}\right)\right)}{\beta^{(1)} \beta^{(2)}} \\
& \text { for } c^{(n)}<\xi_{1}<d^{(n)}(n=1,2),
\end{aligned}
$$

where $\Theta\left(x_{1}, \xi_{1}\right)$ is given in (3.20) and $\Omega_{0}\left(x_{1}, \xi_{1}, \xi_{2}\right)$ is given by

$$
\begin{aligned}
\Omega_{0}\left(x_{1}, \xi_{1}, \xi_{2}\right)= & \frac{\left(x_{1}+\xi_{1}\right)^{2}-\xi_{2}^{2}}{\left(\left(x_{1}+\xi_{1}\right)^{2}+\xi_{2}^{2}\right)^{2}}+\frac{1}{2 L^{2}} \Psi_{1}\left(\frac{L+\xi_{1}+x_{1}-\xi_{2} i}{L}\right) \\
& +\frac{1}{2 L^{2}} \Psi_{1}\left(\frac{L+\xi_{1}+x_{1}+\xi_{2} i}{L}\right)+\frac{1}{2 L^{2}} \Psi_{1}\left(\frac{-L+\xi_{1}+x_{1}-\xi_{2} i}{-L}\right) \\
& +\frac{1}{2 L^{2}} \Psi_{1}\left(\frac{-L+\xi_{1}+x_{1}+\xi_{2} i}{-L}\right)
\end{aligned}
$$

with $\Psi_{1}$ being the trigamma function defined in (2.45).

As $h \rightarrow \infty$, the hypersingular integral equations (4.8) and (4.9) reduce to (3.37) for the case where the micro-cracked interface lies between two dissimilar half-spaces.

For inplane deformations, the conditions on the interface are given by (3.34). With $u_{r}^{(\text {ext) }}$ and $\sigma_{r 2}^{(\text {ext) }}$ assumed known, the boundary value problem here is to solve (2.10) together with (4.1) subject to the conditions (3.34) and (4.5).

To solve the boundary value problem for inplane deformations, the boundary integral equations (2.25) are used together with the perfect interface Green's 
functions (2.37) and (2.40) to derive the integral solution

$$
\begin{aligned}
u_{j}^{(\mathrm{imp})}\left(\xi_{1}, \xi_{2}\right)= & \frac{1}{2 \pi} \sum_{n=-\infty}^{\infty} \int_{n L}^{(n+1) L} u_{r}^{(\mathrm{imp})}\left(x_{1}, h\right) \\
& \times \operatorname{Re}\left\{\sum _ { \alpha = 1 } ^ { 2 } L _ { r 2 \alpha } ^ { ( 1 ) } \left[\frac{N_{\alpha p}^{(1)}}{x_{1}-\xi_{1}+\tau_{\alpha}^{(1)}\left(h-\xi_{2}\right)}\right.\right. \\
& \left.\left.+\sum_{\beta=1}^{2} Q_{\alpha \beta p}^{(1)} \frac{1}{x_{1}-\xi_{1}+\tau_{\alpha}^{(1)} h-\bar{\tau}_{\beta}^{(1)} \xi_{2}}\right]\right\} D_{p j}^{(1)} d x_{1} \\
& -\frac{1}{2 \pi} \sum_{n=-\infty}^{\infty} \sum_{k=1}^{2} \int_{c^{(k)}+n L}^{d^{(k)}+n L} \Delta u_{r}^{(\mathrm{imp})}\left(x_{1}\right) \\
& \times \operatorname{Re}\left\{\sum _ { \alpha = 1 } ^ { 2 } L _ { r 2 \alpha } ^ { ( 1 ) } \left[\frac{N_{\alpha p}^{(1)}}{x_{1}-\xi_{1}-\tau_{\alpha}^{(1)} \xi_{2}}\right.\right. \\
& \left.\left.+\sum_{\beta=1}^{2} Q_{\alpha \beta p}^{(1)} \frac{1}{x_{1}-\xi_{1}-\bar{\tau}_{\beta}^{(1)} \xi_{2}}\right]\right\} D_{p j}^{(1)} d x_{1} \\
& \text { for } 0<\xi_{2}<h,
\end{aligned}
$$

where $\tau_{\alpha}^{(p)}, L_{r 2 \alpha}^{(p)}, N_{\alpha p}^{(p)}$ and $D_{p j}^{(p)}$ are defined below (2.39) and $Q_{\alpha \beta p}^{(1)}$ is implicitly defined in (2.38).

Substitution of (4.11) into the generalized Hooke's law gives rise to the inplane stress formulae

$$
\begin{aligned}
\sigma_{i 2}^{(\mathrm{imp})}\left(\xi_{1}, \xi_{2}\right)= & \frac{1}{2 \pi} \operatorname{Re}\left\{\sum_{n=-\infty}^{\infty} \int_{n L}^{(n+1) L} u_{r}^{(\mathrm{imp})}\left(x_{1}, h\right)\right. \\
& \times\left[\sum_{\alpha=1}^{2} \frac{G_{r i \alpha}}{\left(x_{1}-\xi_{1}+\tau_{\alpha}^{(1)}\left(h-\xi_{2}\right)\right)^{2}}\right. \\
& \left.\left.+\sum_{\alpha=1}^{2} \sum_{\beta=1}^{2} \frac{H_{r i \alpha \beta}}{\left(x_{1}-\xi_{1}+\tau_{\alpha}^{(1)} h-\bar{\tau}_{\beta}^{(1)} \xi_{2}\right)^{2}}\right] d x_{1}\right\}
\end{aligned}
$$




$$
\begin{aligned}
& -\frac{1}{2 \pi} \operatorname{Re}\left\{\sum_{n=-\infty}^{\infty} \sum_{k=1}^{2} \int_{c^{(k)}+n L}^{d^{(k)}+n L} \Delta u_{r}^{(\mathrm{imp})}\left(x_{1}\right)\right. \\
& \times\left[\sum_{\alpha=1}^{2} \frac{G_{r i \alpha}}{\left(x_{1}-\xi_{1}-\tau_{\alpha}^{(1)} \xi_{2}\right)^{2}}\right. \\
& \left.\left.+\sum_{\alpha=1}^{2} \sum_{\beta=1}^{2} \frac{H_{r i \alpha \beta}}{\left(x_{1}-\xi_{1}-\bar{\tau}_{\beta}^{(1)} \xi_{2}\right)^{2}}\right] d x_{1}\right\} \\
& \text { for } 0<\xi_{2}<h
\end{aligned}
$$

where

$$
\begin{aligned}
G_{r i \alpha} & =\left[L_{r 2 \alpha}^{(1)} N_{\alpha p}^{(1)}\left(c_{i 2 m 1}^{(1)}+\tau_{\alpha}^{(1)} c_{i 2 m 2}^{(1)}\right)\right] D_{p m}^{(1)} \\
H_{r i \alpha \beta} & =\left[L_{r 2 \alpha}^{(1)} Q_{\alpha \beta p}^{(1)}\left(c_{i 2 m 1}^{(1)}+\bar{\tau}_{\beta}^{(1)} c_{i 2 m 2}^{(1)}\right)\right] D_{p m}^{(1)}
\end{aligned}
$$

with $c_{i j k l}^{(p)}$ defined below (2.39).

From the summation formula in (2.45) and (4.12), the conditions in (3.22) and (4.5) may be respectively expressed in terms of the hypersingular boundary integral equations

$$
\begin{aligned}
& \quad \frac{1}{2 \pi} f_{0}^{d^{(2)}} u_{r}^{(\mathrm{imp})}\left(x_{1}, h\right) \operatorname{Re}\left\{\sum_{\alpha=1}^{2} G_{r i \alpha}\right\}\left[\frac{1}{\left(x_{1}-\xi_{1}\right)^{2}}+\Theta\left(x_{1}, \xi_{1}\right)\right] d x_{1} \\
& +\frac{1}{2 \pi} \int_{0}^{d^{(2)}} u_{r}^{(\mathrm{imp})}\left(x_{1}, h\right) \operatorname{Re}\left\{\sum_{\alpha=1}^{2} \sum_{\beta=1}^{2} H_{r i \alpha \beta} \Omega\left(x_{1}, \xi_{1}, \tau_{\alpha}^{(1)} h-\bar{\tau}_{\beta}^{(1)} h\right)\right\} d x_{1} \\
& -\frac{1}{2 \pi} \sum_{k=1}^{2} \int_{c^{(k)}}^{d^{(k)}} \Delta u_{r}^{(\mathrm{imp})}\left(x_{1}\right) \operatorname{Re}\left\{\sum_{\alpha=1}^{2} G_{r i \alpha} \Omega\left(x_{1}, \xi_{1},-\tau_{\alpha}^{(1)} h\right)\right\} d x_{1} \\
& -\frac{1}{2 \pi} \sum_{k=1}^{2} \int_{c^{(k)}}^{d^{(k)}} \Delta u_{r}^{(\mathrm{imp})}\left(x_{1}\right) \operatorname{Re}\left\{\sum_{\alpha=1}^{2} \sum_{\beta=1}^{2} H_{r i \alpha \beta} \Omega\left(x_{1}, \xi_{1},-\bar{\tau}_{\beta}^{(1)} h\right)\right\} d x_{1} \\
& =0 \text { for } 0<\xi_{1}<d^{(2)},
\end{aligned}
$$


and the hypersingular boundary integro-differential equations

$$
\begin{aligned}
& -\frac{1}{2 \pi} \int_{0}^{d^{(2)}} u_{r}^{(\mathrm{imp})}\left(x_{1}, h\right) \operatorname{Re}\left\{\sum_{\alpha=1}^{2} \Omega\left(x_{1}, \xi_{1}, \tau_{\alpha}^{(1)} h\right)\left(G_{r i \alpha}+\sum_{\beta=1}^{2} H_{r i \alpha \beta}\right)\right\} d x_{1} \\
& +\frac{1}{2 \pi}\left[f_{c^{(k)}}^{d^{(k)}} \frac{\Delta u_{r}^{(\mathrm{imp})}\left(x_{1}\right) \operatorname{Re}\left\{W_{i r}\right\}}{\left(x_{1}-\xi_{1}\right)^{2}} d x_{1}\right. \\
& +\sum_{\substack{n=1 \\
n \neq k}}^{2} \int_{c^{(n)}}^{d^{(n)}} \frac{\Delta u_{r}^{(\mathrm{imp})}\left(x_{1}\right) \operatorname{Re}\left\{W_{i r}\right\}}{\left(x_{1}-\xi_{1}\right)^{2}} d x_{1} \\
& \left.+\sum_{n=1}^{2} \int_{c^{(n)}}^{d^{(n)}} \Delta u_{r}^{(\mathrm{imp})}\left(x_{1}\right) \operatorname{Re}\left\{W_{i r}\right\} \Theta\left(x_{1}, \xi_{1}\right) d x_{1}-\frac{d \Delta u_{r}^{(\mathrm{imp})}\left(\xi_{1}\right)}{d \xi_{1}} \operatorname{Im}\left\{\pi V_{i r}\right\}\right] \\
= & \delta_{i j} \sigma_{j 2}^{(\mathrm{ext})}\left(\xi_{1}, 0^{+}\right)-\delta^{(k 2)} k_{i j} \Delta u_{j}^{(\mathrm{imp})}\left(\xi_{1}\right) \\
& \text { for } c^{(k)}<\xi_{1}<d^{(k)}(k=1,2),
\end{aligned}
$$

where $\Omega\left(x_{1}, \xi_{1}, z\right)$ is defined by

$$
\begin{aligned}
\Omega\left(x_{1}, \xi_{1}, z\right)= & \frac{1}{\left(x_{1}-\xi_{1}+z\right)^{2}}+\frac{1}{L^{2}} \Psi_{1}\left(\frac{L+x_{1}-\xi_{1}+z}{L}\right) \\
& +\frac{1}{L^{2}} \Psi_{1}\left(\frac{L-x_{1}+\xi_{1}-z}{L}\right),
\end{aligned}
$$

and the constants $W_{i r}$ and $V_{i r}$ are given by

$$
\begin{aligned}
W_{i r} & =\sum_{\alpha=1}^{2} G_{r i \alpha}+\sum_{\alpha=1}^{2} \sum_{\beta=1}^{2} H_{r i \alpha \beta}, \\
V_{i r} & =\sum_{\alpha=1}^{2} \sum_{\beta=1}^{2} H_{r i \alpha \beta}-\sum_{\alpha=1}^{2} G_{r i \alpha} .
\end{aligned}
$$

As $h \rightarrow \infty$, we expect (4.13) and (4.14) to reduce to (3.38) for the case where the interface is between two dissimilar half-spaces. Comparing (3.38) with (4.14) for the limiting case where $h \rightarrow \infty$, we deduce that

$$
\begin{aligned}
& \operatorname{Re}\left\{Q_{i r}\right\}=-\frac{\operatorname{Re}\left\{W_{i r}\right\}}{2 \pi}, \\
& \operatorname{Im}\left\{Q_{i r}\right\}=-\frac{\operatorname{Im}\left\{V_{i r}\right\}}{2 \pi} .
\end{aligned}
$$




\section{Micromechanical Models for Weak Interfaces}

For particular orthotropic materials, we have verified that (4.17) is true.

The hypersingular boundary integro-differential equations in (4.8), (4.9), (4.13) and (4.14) can be discretized into algebraic equations as explained in Subsection 4.4.1. The algebraic equations can then be solved to determine the unknown displacements on the plane boundary $0<x_{1}<d^{(2)}, x_{2}=h$ and the displacement jumps across the damaged parts of the interface and to compute the effective stiffness coefficients $k_{i j}$ (for inplane deformations) and $k_{33}$ (for antiplane deformations). The loads $\sigma_{32}^{(\text {ext })}$ and $\sigma_{r 2}^{(\text {ext })}$ are taken to be constants as given in (3.31). As explained in Subsection 3.4.2, two independent sets of loads are required to determine effective stiffness coefficients for inplane deformations.

\subsubsection{Periodic Model Involving Evenly Distributed Micro- cracks of Equal Length}

The hypersingular boundary integro-differential equations for the periodic model in Subsection 4.2.2 may be derived by modifying the analysis in Subsection 4.3.1.

For antiplane deformations, the hypersingular boundary integral equation for the condition in (4.4) on the external plane boundary is given by

$$
\begin{aligned}
& f_{0}^{L} \frac{u_{3}^{(\mathrm{imp})}\left(x_{1}, h\right)}{\left(x_{1}-\xi_{1}\right)^{2}} d x_{1} \\
& +\int_{0}^{L} u_{3}^{(\mathrm{imp})}\left(x_{1}, h\right)\left[\Theta\left(x_{1}, \xi_{1}\right)-\frac{\beta^{(1)}-\beta^{(2)}}{\beta^{(1)}+\beta^{(2)}} \Omega_{0}\left(x_{1},-\xi_{1}, \frac{2 \beta^{(1)}}{C_{44}^{(1)}} h\right)\right] d x_{1} \\
& -\frac{2 \beta^{(2)}}{\beta^{(1)}+\beta^{(2)}} \int_{a^{(1)}}^{b^{(1)}} \Delta u_{3}^{(\mathrm{imp})}\left(x_{1}\right) \Omega_{0}\left(x_{1},-\xi_{1},-\frac{\beta^{(1)}}{C_{44}^{(1)}} h\right) d x_{1} \\
& =0 \text { for } 0<\xi_{1}<L,
\end{aligned}
$$


and the one for condition (3.6) is given by

$$
\begin{aligned}
& \int_{0}^{L} u_{3}^{(\mathrm{imp})}\left(x_{1}, h\right) \Omega_{0}\left(x_{1},-\xi_{1}, \frac{\beta^{(1)}}{C_{44}^{(1)}} h\right) d x_{1} \\
- & f_{a^{(1)}}^{b^{(1)}} \frac{\Delta u_{3}^{(\mathrm{imp})}\left(x_{1}\right)}{\left(x_{1}-\xi_{1}\right)^{2}} d x_{1} \\
& -\int_{a^{(1)}}^{b^{(1)}} \Delta u_{3}^{(\mathrm{imp})}\left(x_{1}\right) \Theta\left(x_{1}, \xi_{1}\right) d x_{1} \\
= & \frac{\pi\left(\beta^{(1)}+\beta^{(2)}\right)}{\beta^{(1)} \beta^{(2)}} \sigma_{32}^{(\mathrm{ext})}\left(\xi_{1}, 0^{+}\right) \text {for } a^{(1)}<\xi_{1}<b^{(1)} .
\end{aligned}
$$

Note that (4.18) and (4.19) reduce to (3.39) as $h \rightarrow \infty$.

For inplane deformations, the hypersingular boundary integral equations for the conditions in (4.5) can be written as

$$
\begin{aligned}
& \quad \frac{1}{2 \pi} f_{0}^{L} u_{r}^{(\mathrm{imp})}\left(x_{1}, h\right) \operatorname{Re}\left\{\sum_{\alpha=1}^{2} G_{r i \alpha}\right\}\left[\frac{1}{\left(x_{1}-\xi_{1}\right)^{2}}+\Theta\left(x_{1}, \xi_{1}\right)\right] d x_{1} \\
& +\frac{1}{2 \pi} \int_{0}^{L} u_{r}^{(\mathrm{imp})}\left(x_{1}, h\right) \operatorname{Re}\left\{\sum_{\alpha=1}^{2} \sum_{\beta=1}^{2} H_{r i \alpha \beta} \Omega\left(x_{1}, \xi_{1}, \tau_{\alpha}^{(1)} h-\bar{\tau}_{\beta}^{(1)} h\right)\right\} d x_{1} \\
& -\frac{1}{2 \pi} \int_{a^{(1)}}^{b^{(1)}} \Delta u_{r}^{(\mathrm{imp})}\left(x_{1}\right) \operatorname{Re}\left\{\sum_{\alpha=1}^{2} G_{r i \alpha} \Omega\left(x_{1}, \xi_{1},-\tau_{\alpha}^{(1)} h\right)\right\} d x_{1} \\
& -\frac{1}{2 \pi} \int_{a^{(1)}}^{b^{(1)}} \Delta u_{r}^{(\mathrm{imp})}\left(x_{1}\right) \operatorname{Re}\left\{\sum_{\alpha=1}^{2} \sum_{\beta=1}^{2} H_{r i \alpha \beta} \Omega\left(x_{1}, \xi_{1},-\bar{\tau}_{\beta}^{(1)} h\right)\right\} d x_{1} \\
& =0 \text { for } 0<\xi_{1}<L,
\end{aligned}
$$

and the hypersingular boundary integro-differential equations for conditions (3.21) are given by

$$
-\frac{1}{2 \pi} \int_{0}^{L} u_{r}^{(\mathrm{imp})}\left(x_{1}, h\right) \operatorname{Re}\left\{\sum_{\alpha=1}^{2} \Omega\left(x_{1}, \xi_{1}, \tau_{\alpha}^{(1)} h\right)\left(G_{r i \alpha}+\sum_{\beta=1}^{2} H_{r i \alpha \beta}\right)\right\} d x_{1}
$$




$$
\begin{aligned}
& \quad+\frac{1}{2 \pi}\left[f_{a^{(1)}}^{b^{(1)}} \frac{\Delta u_{r}^{(\mathrm{imp})}\left(x_{1}\right) \operatorname{Re}\left\{W_{i r}\right\}}{\left(x_{1}-\xi_{1}\right)^{2}} d x_{1}\right. \\
& \left.+\int_{a^{(1)}}^{b^{(1)}} \Delta u_{r}^{(\mathrm{imp})}\left(x_{1}\right) \operatorname{Re}\left\{W_{i r}\right\} \Theta\left(x_{1}, \xi_{1}\right) d x_{1}-\frac{d \Delta u_{r}^{(\mathrm{imp})}\left(\xi_{1}\right)}{d \xi_{1}} \operatorname{Im}\left\{\pi V_{i r}\right\}\right] \\
& =\delta_{i j} \sigma_{j 2}^{(\mathrm{ext})}\left(\xi_{1}, 0^{+}\right) \text {for } a^{(1)}<\xi_{1}<b^{(1)} .
\end{aligned}
$$

As $h \rightarrow \infty,(4.20)$ and (4.21) reduce to (3.40) with the constants $Q_{i r}$ related to $W_{i r}$ and $V_{i r}$ by (4.17).

The hypersingular boundary integro-differential equations (4.18), (4.19), (4.20) and (4.21) can be discretized into linear algebraic equations as explained in Subsection 4.4.2 to solve for the unknown displacements on the plane boundary $0<x_{1}<L, x_{2}=h$ and for the displacement jumps across the micro-crack $a^{(1)}<x_{1}<b^{(1)}, x_{2}=0$. Once the displacement jumps are determined, the effective stiffness coefficients can be readily calculated as explained in Subsection 3.4 .2 .

\subsubsection{Micromechanical-statistical Model}

In the micromechanical-statistical model, $N$ interfaces for selected parameters are randomly generated to form a statistical sample. For each interface in the sample, the analysis in Subsection 4.3 .1 can be easily modified to derive hypersingular boundary integro-differential equations for the conditions over a period interval of the interface.

For antiplane deformations, the hypersingular boundary integral equations for the micromechanical-statistical model are given by

$$
\begin{aligned}
& f_{0}^{L} \frac{u_{3}^{(\mathrm{imp})}\left(x_{1}, h\right)}{\left(x_{1}-\xi_{1}\right)^{2}} d x_{1} \\
& +\int_{0}^{L} u_{3}^{(\mathrm{imp})}\left(x_{1}, h\right)\left[\Theta\left(x_{1}, \xi_{1}\right)-\frac{\beta^{(1)}-\beta^{(2)}}{\beta^{(1)}+\beta^{(2)}} \Omega_{0}\left(x_{1},-\xi_{1}, \frac{2 \beta^{(1)}}{C_{44}^{(1)}} h\right)\right] d x_{1}
\end{aligned}
$$




$$
\begin{aligned}
& -\frac{2 \beta^{(2)}}{\beta^{(1)}+\beta^{(2)}} \sum_{m=1}^{M} \int_{a^{(m)}}^{b^{(m)}} \Delta u_{3}^{(\mathrm{imp})}\left(x_{1}\right) \Omega_{0}\left(x_{1},-\xi_{1},-\frac{\beta^{(1)}}{C_{44}^{(1)}} h\right) d x_{1} \\
= & 0 \text { for } 0<\xi_{1}<L,
\end{aligned}
$$

and

$$
\begin{aligned}
& \int_{0}^{L} u_{3}^{(\mathrm{imp})}\left(x_{1}, h\right) \Omega_{0}\left(x_{1},-\xi_{1}, \frac{\beta^{(1)}}{C_{44}^{(1)}} h\right) d x_{1} \\
& -f_{a^{(n)}}^{b^{(n)}} \frac{\Delta u_{3}^{(\mathrm{imp})}\left(x_{1}\right)}{\left(x_{1}-\xi_{1}\right)^{2}} d x_{1}-\sum_{\substack{m=1 \\
m \neq n}}^{M} \int_{a^{(m)}}^{b^{(m)}} \frac{\Delta u_{3}^{(\mathrm{imp})}\left(x_{1}\right)}{\left(x_{1}-\xi_{1}\right)^{2}} d x_{1} \\
& -\sum_{m=1}^{M} \int_{a^{(m)}}^{b^{(m)}} \Delta u_{3}^{(\mathrm{imp})}\left(x_{1}\right) \Theta\left(x_{1}, \xi_{1}\right) d x_{1} \\
= & \frac{\pi\left(\beta^{(1)}+\beta^{(2)}\right)}{\beta^{(1)} \beta^{(2)}} \sigma_{32}^{(\mathrm{ext})}\left(\xi_{1}, 0^{+}\right) \text {for } a^{(n)}<\xi_{1}<b^{(n)}(n=1,2, \cdots, M) .
\end{aligned}
$$

For inplane deformations, the hypersingular boundary integro-differential equations are given by

$$
\begin{aligned}
& \frac{1}{2 \pi} f_{0}^{L} u_{r}^{(\mathrm{imp})}\left(x_{1}, h\right) \operatorname{Re}\left\{\sum_{\alpha=1}^{2} G_{r i \alpha}\right\}\left[\frac{1}{\left(x_{1}-\xi_{1}\right)^{2}}+\Theta\left(x_{1}, \xi_{1}\right)\right] d x_{1} \\
+ & \frac{1}{2 \pi} \int_{0}^{L} u_{r}^{(\mathrm{imp})}\left(x_{1}, h\right) \operatorname{Re}\left\{\sum_{\alpha=1}^{2} \sum_{\beta=1}^{2} H_{r i \alpha \beta} \Omega\left(x_{1}, \xi_{1}, \tau_{\alpha}^{(1)} h-\bar{\tau}_{\beta}^{(1)} h\right)\right\} d x_{1} \\
& -\frac{1}{2 \pi} \sum_{k=1}^{M} \int_{a^{(k)}}^{b^{(k)}} \Delta u_{r}^{(\mathrm{imp})}\left(x_{1}\right) \operatorname{Re}\left\{\sum_{\alpha=1}^{2} G_{r i \alpha} \Omega\left(x_{1}, \xi_{1},-\tau_{\alpha}^{(1)} h\right)\right\} d x_{1} \\
& -\frac{1}{2 \pi} \sum_{k=1}^{M} \int_{a^{(k)}}^{b^{(k)}} \Delta u_{r}^{(\mathrm{imp})}\left(x_{1}\right) \operatorname{Re}\left\{\sum_{\alpha=1}^{2} \sum_{\beta=1}^{2} H_{r i \alpha \beta} \Omega\left(x_{1}, \xi_{1},-\bar{\tau}_{\beta}^{(1)} h\right)\right\} d x_{1} \\
= & 0 \text { for } 0<\xi_{1}<L,
\end{aligned}
$$


and

$$
\begin{aligned}
& -\frac{1}{2 \pi} \int_{0}^{L} u_{r}^{(\mathrm{imp})}\left(x_{1}, h\right) \operatorname{Re}\left\{\sum_{\alpha=1}^{2} \Omega\left(x_{1}, \xi_{1}, \tau_{\alpha}^{(1)} h\right)\left(G_{r i \alpha}+\sum_{\beta=1}^{2} H_{r i \alpha \beta}\right)\right\} d x_{1} \\
& +\frac{1}{2 \pi}\left[f_{a^{(k)}}^{b^{(k)}} \frac{\Delta u_{r}^{(\mathrm{imp})}\left(x_{1}\right) \operatorname{Re}\left\{W_{i r}\right\}}{\left(x_{1}-\xi_{1}\right)^{2}} d x_{1}\right. \\
& +\sum_{\substack{n=1 \\
n \neq k}}^{M} \int_{a^{(n)}}^{b^{(n)}} \frac{\Delta u_{r}^{(\mathrm{imp})}\left(x_{1}\right) \operatorname{Re}\left\{W_{i r}\right\}}{\left(x_{1}-\xi_{1}\right)^{2}} d x_{1} \\
& \left.+\sum_{n=1}^{M} \int_{a^{(n)}}^{b^{(n)}} \Delta u_{r}^{(\mathrm{imp})}\left(x_{1}\right) \operatorname{Re}\left\{W_{i r}\right\} \Theta\left(x_{1}, \xi_{1}\right) d x_{1}-\frac{d \Delta u_{r}^{(\mathrm{imp})}\left(\xi_{1}\right)}{d \xi_{1}} \operatorname{Im}\left\{\pi V_{i r}\right\}\right] \\
= & \delta_{i j} \sigma_{j 2}^{\text {(ext) }}\left(\xi_{1}, 0^{+}\right) \text {for } a^{(k)}<\xi_{1}<b^{(k)}(k=1,2, \ldots, M) .
\end{aligned}
$$

As $h \rightarrow \infty,(4.22)$ and (4.23) for antiplane deformations reduce to (3.19), and (4.24) and (4.25) for inplane deformations reduce to (3.30).

For each interface in the statistical sample, the hypersingular boundary integro-differential equations (4.22), (4.23), (4.24) and (4.25) can be reduced to linear algebraic equations by using the numerical procedures given in Subsection 4.4.3 for solving the unknowns $u_{3}^{(\mathrm{imp})}\left(x_{1}, h\right)$ and $u_{r}^{(\mathrm{imp})}\left(x_{1}, h\right)$ on the external plane boundary and $\Delta u_{3}^{(\mathrm{imp})}\left(x_{1}\right)$ and $\Delta u_{r}^{(\mathrm{imp})}\left(x_{1}\right)$ over the micro-cracks. Once the displacement jumps over the micro-cracks are obtained, the effective stiffness coefficients for each interface may be estimated as explained in Subsection 3.4.3. For the statistical sample, a set of $N$ data can be obtained for each of the effective stiffness coefficients. If the effective stiffness coefficients are non-dimensionalized appropriately, the mean value and the standard deviation for each non-dimensionalized effective stiffness coefficient calculated for the $N$ interfaces in the sample are calculated by using (3.46) and (3.47). 


\subsection{Numerical Procedures}

Numerical procedures for solving the hypersingular boundary integro-differential equations in Section 4.3 are outlined here.

To treat the integrals involving the unknown functions $u_{3}^{(\mathrm{imp})}\left(x_{1}, h\right)$ and $u_{r}^{(\mathrm{imp})}\left(x_{1}, h\right)$ on the external plane boundary of the thin layer, the region $0 \leq$ $x_{1} \leq L, x_{2}=h$ is divided up into $N_{\mathrm{t}}$ equal subintervals. The $p$-th subinterval is given by $x_{\mathrm{t}}^{(p)} \leq x_{1} \leq x_{\mathrm{t}}^{(p+1)}$.

For antiplane deformations, the unknown function $u_{3}^{(\mathrm{imp})}\left(x_{1}, h\right)$ over each of the subintervals is approximated as linear functions of $x_{1}$, that is,

$$
u_{3}^{(\mathrm{imp})}\left(x_{1}, h\right) \simeq x_{1} \phi^{(p)}+\psi^{(p)} \text { for } x_{\mathrm{t}}^{(p)} \leq x_{1} \leq x_{\mathrm{t}}^{(p+1)}
$$

where $\phi^{(p)}$ and $\psi^{(p)}$ are $2 N_{\mathrm{t}}$ unknown constants to be determined.

For inplane deformations, the unknown functions $u_{r}^{(\mathrm{imp})}\left(x_{1}, h\right)$ are approximated by

$$
u_{r}^{(\mathrm{imp})}\left(x_{1}, h\right) \simeq x_{1} \phi_{r}^{(p)}+\psi_{r}^{(p)} \text { for } x_{\mathrm{t}}^{(p)} \leq x_{1} \leq x_{\mathrm{t}}^{(p+1)},
$$

where $\phi_{r}^{(p)}$ and $\psi_{r}^{(p)}$ are $4 N_{\mathrm{t}}$ unknown constants to be determined.

\subsubsection{Three-phase Model}

The hypersingular boundary integro-differential equations (4.8), (4.9), (4.13) and (4.14) for the three-phase model are approximated as algebraic equations as explained below.

For antiplane deformations, (4.26) is used to approximate the unknown function $u_{3}^{(\mathrm{imp})}\left(x_{1}, h\right)$ over the $p$-th element on the external plane boundary in (4.8) and (4.9). The antiplane displacement jump $\Delta u_{3}^{(\mathrm{imp})}\left(x_{1}\right)$ over the representative micro-crack $c^{(1)}<x_{1}<d^{(1)}$ is approximated by (3.48) as given 


\section{Micromechanical Models for Weak Interfaces}

in Subsection 3.5.1. The effective region $c^{(2)}<x_{1}<d^{(2)}$ is discretized into $N_{\mathrm{e}}$ equal subintervals given by (3.49) and (3.50). The displacement jump $\Delta u_{3}^{(\mathrm{imp})}\left(x_{1}\right)$ over the $p$-th element on the effective region is approximated by using (3.51).

With the above approximations, (4.8) and (4.9) can be collocated at $2 N_{\mathrm{t}}+$ $N_{\mathrm{c}}+2 N_{\mathrm{e}}$ collocation points to obtain $2 N_{\mathrm{t}}+N_{\mathrm{c}}+2 N_{\mathrm{e}}$ algebraic equations. We collocate (4.8) at $2 N_{\mathrm{t}}$ collocations over the elements on the plane boundary $x_{2}=h$. The two collocation points on the $p$-th element on the plane boundary are given by

$$
\left.\begin{array}{rl}
\left(\widetilde{x}_{\mathrm{t}}^{(p)}, h\right) & =\left(\frac{3}{4} x_{\mathrm{t}}^{(p)}+\frac{1}{4} x_{\mathrm{t}}^{(p+1)}, h\right) \\
\left(\widetilde{x}_{\mathrm{t}}^{\left(p+N_{\mathrm{t}}\right)}, h\right) & =\left(\frac{1}{4} x_{\mathrm{t}}^{(p)}+\frac{3}{4} x_{\mathrm{t}}^{(p+1)}, h\right)
\end{array}\right\} \text { for } p=1,2, \ldots, N_{\mathrm{t}} .
$$

The collocation points given in (3.52) are used for collocating the hypersingular boundary integro-differential equations (4.9) at $N_{\mathrm{c}}$ points on the representative micro-crack. The points given by (3.54) are used for collocating (4.9) at $2 N_{\mathrm{e}}$ points on the effective region.

The $2 N_{\mathrm{t}}+N_{\mathrm{c}}+2 N_{\mathrm{e}}$ algebraic equations contain $2 N_{\mathrm{t}}+N_{\mathrm{c}}+2 N_{\mathrm{e}}+1$ unknown constants. The effective stiffness $k_{33}$ is one of these unknowns. The $2 N_{\mathrm{t}}+$ $N_{\mathrm{c}}+2 N_{\mathrm{e}}$ equations are to be solved together with (3.57) for all the unknowns including $k_{33}$. This can be done as explained in Subsection 3.5.1 by using an iterative procedure.

For inplane deformations, (4.27) is used to approximate the unknown functions $u_{r}^{(\mathrm{imp})}\left(x_{1}, h\right)$ over the $p$-th element on the plane boundary $x_{2}=h$ in (4.13) and (4.14). The displacement jumps $\Delta u_{r}^{(\mathrm{imp})}\left(x_{1}\right)$ over the representative micro-crack $c^{(1)}<x_{1}<d^{(1)}$ are approximated by (3.58) as given in Subsection 3.5.1. The effective region $c^{(2)}<x_{1}<d^{(2)}$ is discretized into $N_{\mathrm{e}}$ subintervals as in the case for antiplane deformations. The displacement jumps $\Delta u_{r}^{(\mathrm{imp})}\left(x_{1}\right)$ 
over the $p$-th element on the effective region are approximated by using (3.51).

For two independent sets of external loads, the hypersingular boundary integral equations in (4.13) can be collocated at the points defined by (4.28) to obtain $8 N_{\mathrm{t}}$ algebraic equations and the hypersingular boundary integrodifferential equations in (4.14) at the points given by (3.52) and (3.54) to form another $4 N_{\mathrm{c}}+8 N_{\mathrm{e}}$ algebraic equations. There are $8 N_{\mathrm{t}}+4 N_{\mathrm{c}}+8 N_{\mathrm{e}}+4$ unknowns in the algebraic equations. The unknowns include four effective stiffness coefficients $k_{i j}(i, j=1,2)$. The algebraic equations are to be solved iteratively together with the four equations in (3.62) to determine the effective stiffness coefficients.

\subsubsection{Periodic Model Involving Evenly Distributed Micro- cracks of Equal Length}

For antiplane deformations, if we substitute the approximations (3.63) and (4.26) into (4.18) and (4.19) and collocate the resulting equations of (4.18) and (4.19) respectively at the collocation points given by (4.28) and (3.64), we find that (4.18) and (4.19) reduce approximately to $2 N_{\mathrm{t}}+N_{\mathrm{c}}$ linear algebraic equations containing $2 N_{\mathrm{t}}+N_{\mathrm{c}}$ unknowns. Once the displacement jump $\Delta u_{3}^{(\mathrm{imp})}\left(x_{1}\right)$ is obtained by solving the linear algebraic equations, the effective stiffness coefficient $k_{33}$ of the interface may then be calculated readily by using (3.66).

For inplane deformations, substitution of the approximations (4.27) and (3.67) into (4.20) and (4.21) and collocating the resulting equations of (4.20) and (4.21) respectively at the collocation points given by (4.28) and (3.64) give rise to a system of $8 N_{\mathrm{t}}+4 N_{\mathrm{c}}$ linear algebraic equations for two independent sets of external loads. Once the displacement jumps over the micro-cracks are obtained by solving the linear algebraic equations, we may estimate the 
effective stiffness coefficients $k_{i j}$ by using (3.69).

\subsubsection{Micromechanical-statistical Model}

For antiplane deformations, if we substitute the approximations (3.70) and (4.26) into the hypersingular boundary integro-differential equations (4.22) and (4.23) and collocate the resulting equations of (4.22) and (4.23) respectively at the collocation points given by (4.28) and (3.71), (4.22) and (4.23) are reduced to a system of $2 N_{\mathrm{t}}+N_{\mathrm{c}}^{(1)}+N_{\mathrm{c}}^{(2)}+\ldots+N_{\mathrm{c}}^{(M)}$ linear algebraic equations. Once the

displacement jumps $\Delta u_{3}^{(\mathrm{imp})}\left(x_{1}\right)$ over the micro-cracks are obtained by solving the linear algebraic equations, the effective stiffness $k_{33}$ may be estimated readily by using (3.73).

For inplane deformations, substitution of the approximations (3.74) and (4.27) into the hypersingular boundary integro-differential equations (4.24) and (4.25) and collocating the resulting equations of (4.24) and (4.25) respectively at the collocation points given by (4.28) and (3.71) give rise to a system of $8 N_{\mathrm{t}}+4 N_{\mathrm{c}}^{(1)}+4 N_{\mathrm{c}}^{(2)}+\ldots+4 N_{\mathrm{c}}^{(M)}$ linear algebraic equations for two independent sets of loads. Once the displacement jumps over the micro-cracks are obtained by solving the linear algebraic equations, the effective stiffness coefficients $k_{i j}$ may be estimated simultaneously by using (3.76).

\subsubsection{Numerical Values of Effective Stiffness Coefficients}

As in Sections 3.6 and 3.7, all the numerical values of the inplane effective stiffness coefficients obtained in Sections 4.5 and 4.6 show that $k_{11}$ and $k_{22}$ are comparable in magnitudes but $k_{12}$ and $k_{21}$ are insignificant in magnitude compared to either $k_{11}$ or $k_{22}$. Specifically, the values of $\left|k_{12} / k_{11}\right|$ and $\left|k_{21} / k_{22}\right|$ are found to be of the order $10^{-5}$ or smaller. Thus, for all the three models here, $k_{12}$ and $k_{21}$ are regarded as approximately equal to zero in Sections 4.5 
and 4.6 below.

\subsection{Numerical Comparison between the Three- phase and the Periodic Models}

The values of the effective stiffness coefficients $k_{i j}$ (for inplane deformations) and $k_{33}$ (for antiplane deformations) obtained by using the three-phase model (in Subsection 4.4.1) are compared here with the corresponding ones obtained using the periodic model (in Subsection 4.4.2). The two sets of values should agree very closely with each other.

\subsubsection{Antiplane Deformations}

For comparing the numerical procedures in Subsections 4.4.1 and 4.4.2 for the case of antiplane deformations, we take $C_{44}^{(p)}=C_{55}^{(p)}=G^{(p)}$, where $G^{(1)}$ and $G^{(2)}$ are the shear modulus of the thin layer and the half-space respectively. The non-dimensionalized effective stiffness $a k_{33} / G^{(2)}$ depends on the damage ratio $\rho$ of the interface, $a / h$ and $G^{(1)} / G^{(2)}$, where $a$ is the half length of the micro-cracks in the three-phase and the periodic models.

Figure 4.4 plots the non-dimensionalized effective stiffness $a k_{33} / G^{(2)}$ for the three-phase and the periodic models against the damage ratio $\rho$ for selected $a / h$ and $G^{(1)} / G^{(2)}=1$. As expected, the plots obtained by the numerical procedure for the three-phase model in Subsection 4.4.1 are close to those calculated by the procedure for the periodic model in Subsection 4.4.2. Further investigations using other values of $G^{(1)} / G^{(2)}$ show a very small difference between the effective stiffness calculated by the two numerical procedures. For fixed values of $a / h$ and the shear modulus ratio $G^{(1)} / G^{(2)}$, the percentage difference of the effective stiffness between the two models in Subsections 4.4.1 and 4.4.2 tends 
to become larger as $\rho$ increases. For $0.1 \leq \rho \leq 0.8$, the value of percentage difference between the two sets of numerical values of $a k_{33} / G^{(2)}$ is less than $3.5 \%$, while for $\rho=0.9$ the value of percentage difference is less than $4.9 \%$.

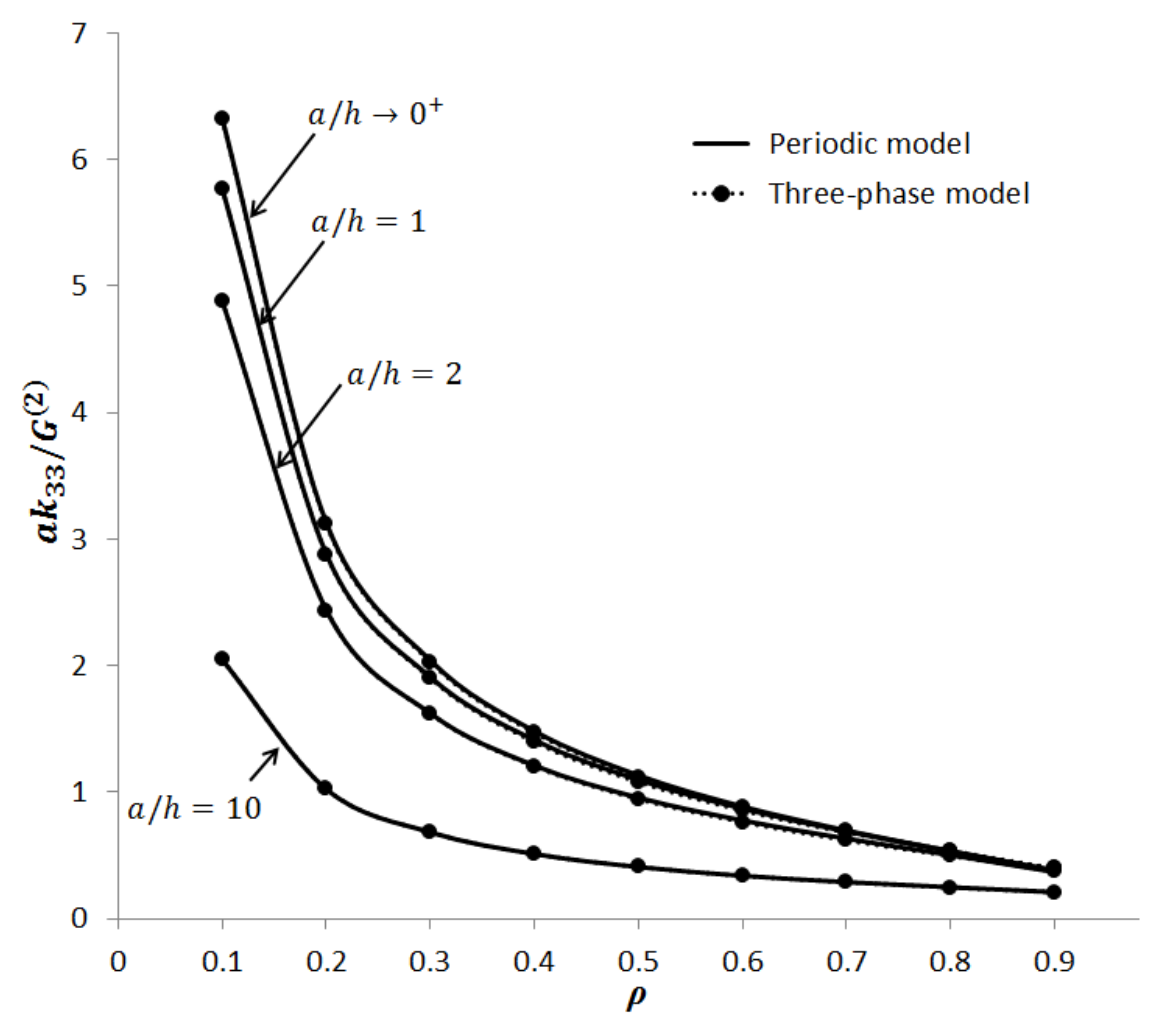

Figure 4.4: Plots of $a k_{33} / G^{(2)}$ against $\rho$ for the shear modulus ratio $G^{(1)} / G^{(2)}=$ 1 and selected values of $a / h$.

For a fixed $\rho$, Figure 4.4 shows that $a k_{33} / G^{(2)}$ becomes larger with decreasing $a / h$. This is expected, as the interaction between the external plane boundary and the interface becomes weaker and gives rise to smaller displacement jumps over the micro-cracks when the values of $a / h$ are relatively small.

More results are given in Figure 4.5, where the damage ratio is fixed as $\rho=0.5$ and $a k_{33} / G^{(2)}$ is plotted against $a / h$ for various selected values of $G^{(1)} / G^{(2)}$. It is observed that each of the plots for the non-dimensionalized ef- 
fective stiffness tends to a constant value as $a / h \rightarrow 0^{+}$. That constant value is very close to the corresponding values of the non-dimensionalized effective stiffness computed using the models in Chapter 3 for a microscopically damaged interface between two dissimilar half-spaces. For a fixed $a / h$, Figure 4.5 also shows that the non-dimensionalized effective stiffness appears to be smaller for a smaller shear modulus ratio $G^{(1)} / G^{(2)}$. This suggests that the damaged interface is "strengthened" and has a greater effective stiffness when the shear modulus $G^{(1)}$ of the thin layer increases relative to the shear modulus $G^{(2)}$ of the half-space.

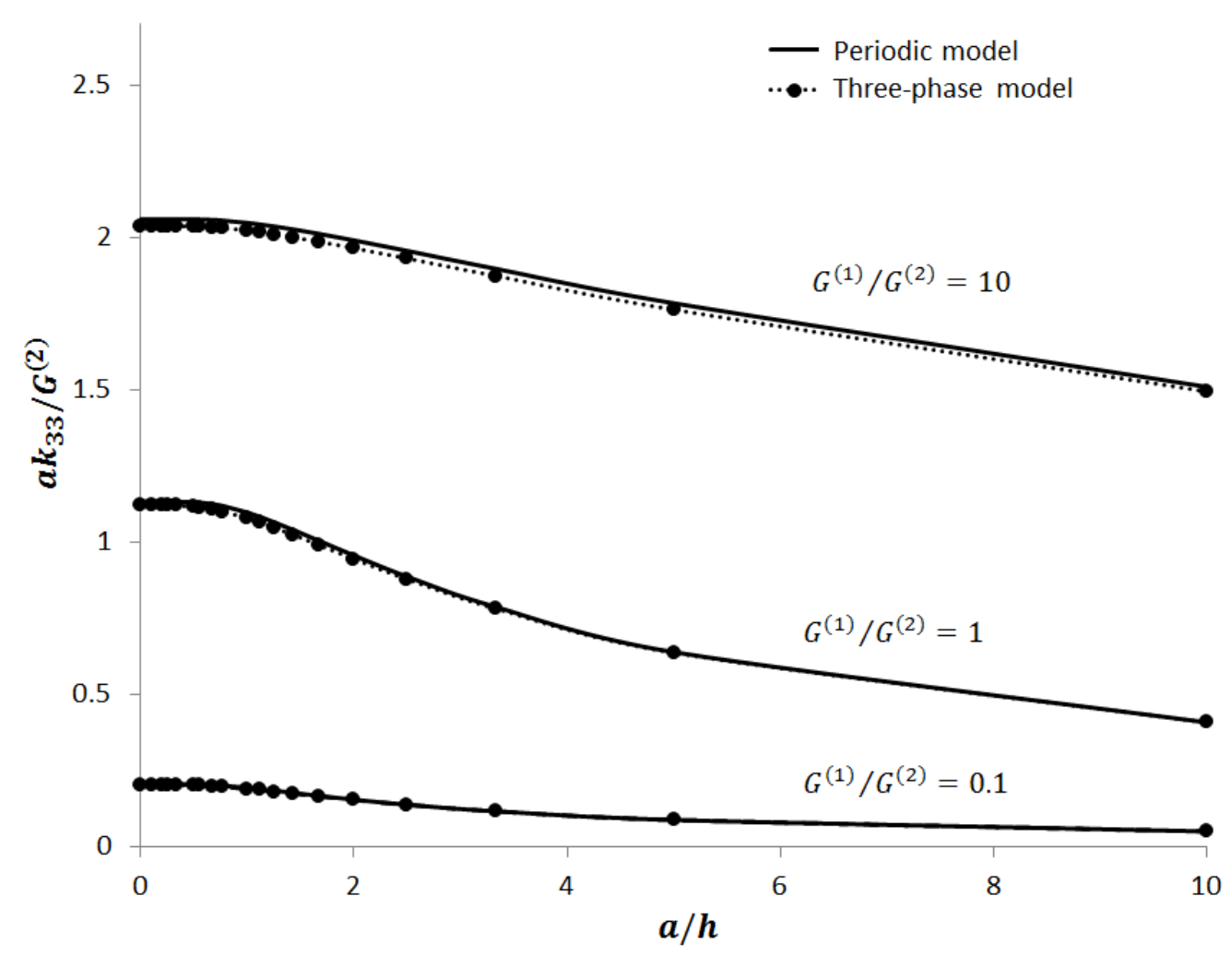

Figure 4.5: Plots of $a k_{33} / G^{(2)}$ against $a / h$ for the damage ratio $\rho=0.5$ and selected values of the shear modulus ratio $G^{(1)} / G^{(2)}$. 


\subsubsection{Inplane Deformations}

To check whether the effective stiffness coefficients $k_{i j}$ obtained by the threephase model agree with those obtained by the periodic model for a particular combination of materials, the elastic moduli (in GPa) for graphite-epoxy and E-glass-epoxy, as given in Table 3.1, are used as the material constants for the orthotropic thin layer and half-space respectively.

Figure 4.6 gives the plots of the non-dimensionalized effective stiffness coefficients $a k_{11} / C_{66}^{(2)}$ and $a k_{22} / C_{66}^{(2)}$ against $a / h$ for the damage ratio $\rho=0.3$, 0.5 and 0.7. As expected, the non-dimensionalized effective stiffness coefficients calculated using the periodic model agree well with the corresponding values predicted by the three-phase model. The percentage difference of the nondimensionalized effective stiffness coefficients between the two models is found to range from $0.012 \%$ to $5.5 \%$ for $0<a / h \leq 5$ and $0.1 \leq \rho \leq 0.9$, with the percentage difference becoming larger as the damage ratio $\rho$ increases.

From Figure 4.6, for a fixed value of $a / h$, each of the non-dimensionalized effective stiffness coefficients $a k_{11} / C_{66}^{(2)}$ and $a k_{22} / C_{66}^{(2)}$ is observed to decrease in magnitude as the damage ratio $\rho$ increases. This is because the coplanar interfacial micro-cracks for larger value of $\rho$ are closer to one another and interact more strongly with one another in such a way that the magnitudes of the displacement jumps increase. It is also observed that $a k_{11} / C_{66}^{(2)}$ and $a k_{22} / C_{66}^{(2)}$ decrease as $a / h$ increases. For a larger value of $a / h$, the external plane boundary of the thin layer is closer to the micro-cracks, giving rise to larger displacement jumps across the micro-damaged parts of the interface. Note that $a / h \rightarrow 0^{+}$corresponds to the case where the bimaterial is made up of two elastic half-spaces. 
(a)

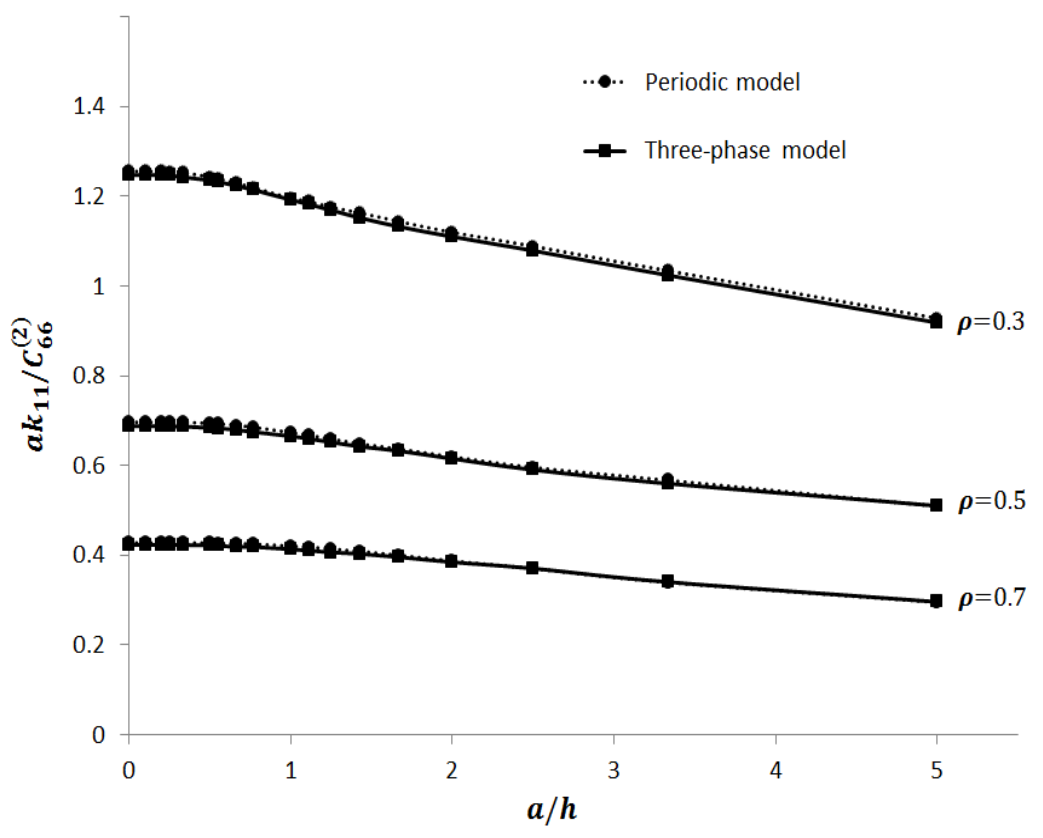

(b)

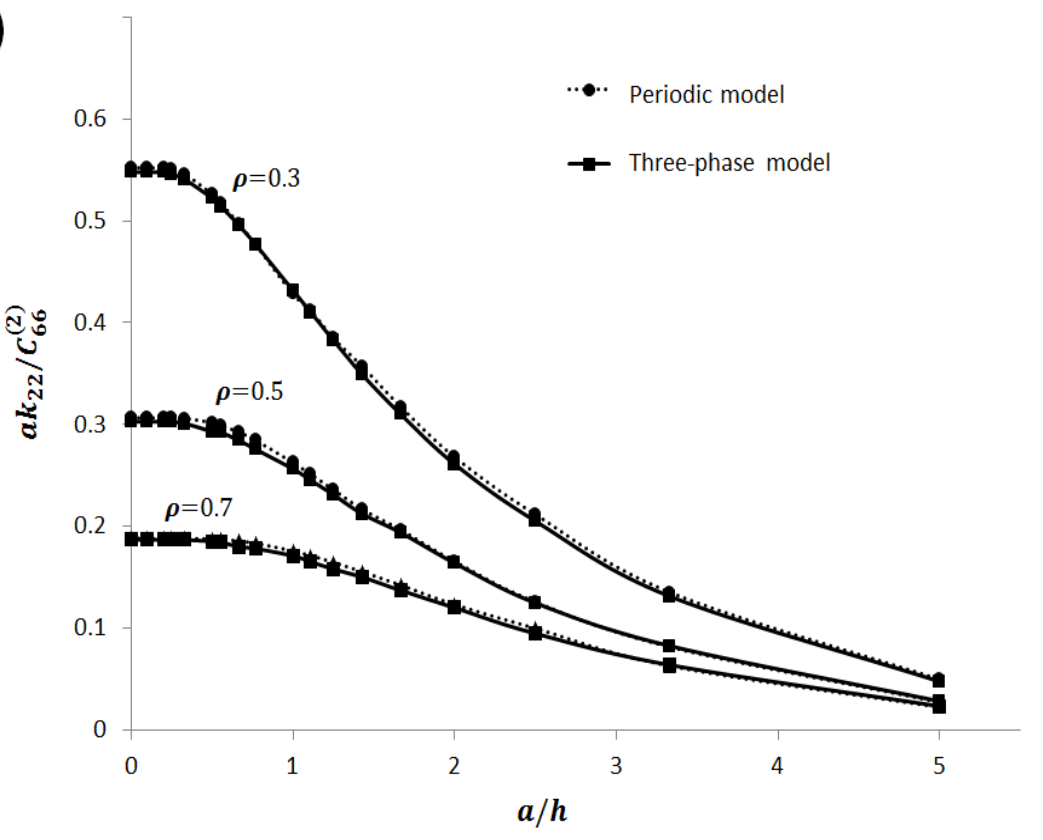

Figure 4.6: Plots of $a k_{11} / C_{66}^{(2)}$ and $a k_{22} / C_{66}^{(2)}$ against $a / h$ for selected values of $\rho$. 


\subsection{Micromechanical-statistical Simulations}

In this section, the micromechanical-statistical model in Subsection 4.2.3 is used to estimate the effective stiffness of a micro-cracked interface for cases where the thin layer and the half-space are occupied by particular materials.

As described in Subsection 4.2.3, an interface is formed by choosing the $M$ micro-cracks over a period interval of the interface to have randomly generated sizes. More specifically, the length of a micro-crack follows a $\chi^{2}$ distribution of a fixed degree of freedom. The $M$ micro-cracks are then randomly positioned over a period length $L$ of the interface. For fixed values of $\rho, \widehat{a} / h$ ( $\widehat{a}$ is the half crack length defined in (3.43)) and material constants in the thin layer and the half-space, $N$ interfaces are randomly generated to form a sample for generating statistical data for the effective stiffness coefficients $k_{i j}$ (for inplane deformations) and $k_{33}$ (for antiplane deformations). As in Subsection 4.5.1, the simulations for antiplane deformations in this section are carried out for the isotropic bimaterial characterized by the shear modulus ratio $G^{(1)} / G^{(2)}$.

If the effective stiffness coefficients for the $n$-th interface are denoted as $k_{11}^{(n)}$, $k_{22}^{(n)}$ and $k_{33}^{(n)}$, we may non-dimensionalize the effective stiffness coefficients in a similar manner as in Section 4.5, that is, the non-dimensionalized effective stiffness coefficients are given by

$$
\begin{aligned}
& K_{11}^{(n)}=\widehat{a}^{(n)} k_{11}^{(n)} / C_{66}^{(2)}, \\
& K_{22}^{(n)}=\widehat{a}^{(n)} k_{22}^{(n)} / C_{66}^{(2)}, \\
& K_{33}^{(n)}=\widehat{a}^{(n)} k_{33}^{(n)} / G^{(2)},
\end{aligned}
$$

where $\widehat{a}^{(n)}$ is the average half crack length defined above (3.77) for the $n$-th interface. The mean values $\widehat{K}_{11}, \widehat{K}_{22}$ and $\widehat{K}_{33}$ of the non-dimensionalized effective stiffness coefficients of the $N$ randomly generated interfaces are calculated 
by using (3.46) and (3.47).

\subsubsection{Number of Micro-cracks for Homogenizing the In- terface}

The number of micro-cracks required per period interval of the interface for homogenizing the effective stiffness coefficients is investigated here by randomly generating 50 micro-cracked interfaces for fixed values of $\rho$ and $\widehat{a} / h$. The $M$ micro-crack lengths follow the $\chi^{2}(5)$ distribution. For each of the interfaces, the mean values $\widehat{K}_{11}, \widehat{K}_{22}$ (for inplane deformations) and $\widehat{K}_{33}$ (for antiplane deformations) of the non-dimensionalized effective stiffness coefficients are calculated. The mean values $\widehat{K}_{11}, \widehat{K}_{22}$ and $\widehat{K}_{33}$ from the 50 interfaces should not vary much when $M$ exceeds a certain value $M_{0}$, which is taken to be the number of micro-cracks required for homogenizing the interface. For inplane deformations, the thin layer and the half-space are respectively occupied by graphite-epoxy and E-glass-expoxy.

For $\rho=0.5$ and $\widehat{a} / h=1$, Figure 4.7 gives the scatter plots of the data for non-dimensionalized effective stiffness coefficients of the 50 interfaces against various values of $M$. The means $\widehat{K}_{11}, \widehat{K}_{22}$ and $\widehat{K}_{33}$ are also indicated in Figure 4.7 for the different values of $M$. Note that the graphs in Figure 4.7 (c) for antiplane deformations are plotted for the shear modulus ratio given by $G^{(1)} / G^{(2)}=1$. The plots of the non-dimensionalized effective stiffness coefficients for antiplane and inplane deformations in Figure 4.7 show the similar observations to the corresponding plots in Figure 3.6. It appears that 40 microcracks per period interval of the interface may be sufficient for homogenizing the non-dimensionalized effective stiffness coefficients here. Similar observations apply for various other values of material constants for the bimaterial, $\rho$ and $\widehat{a} / h$ and for micro-cracks generated using a $\chi^{2}$ distribution of a different 
(a)

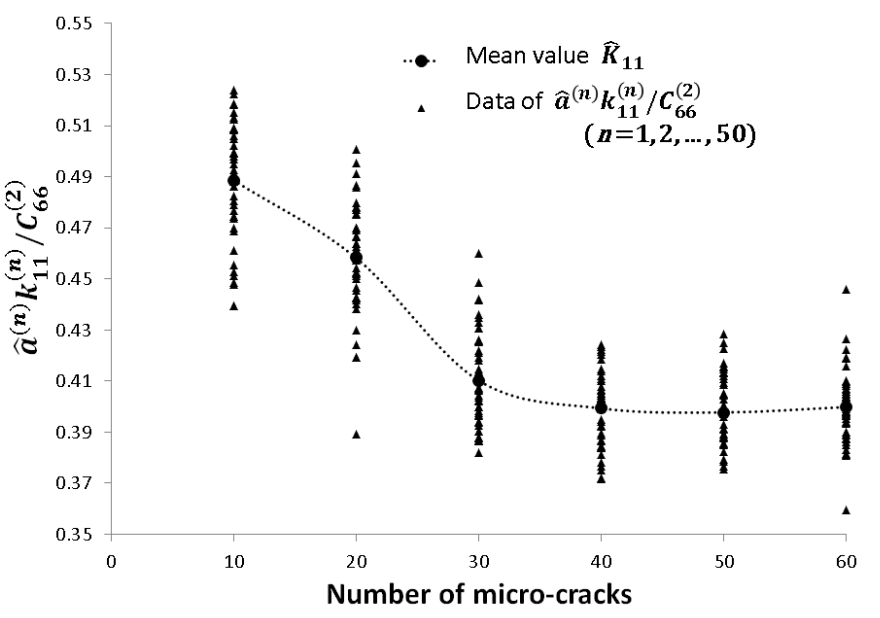

(b)

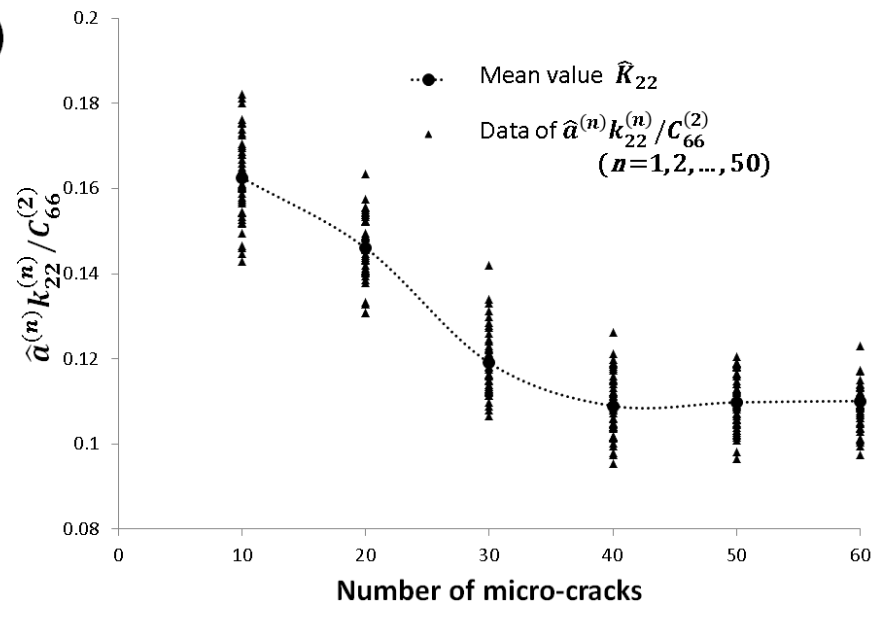

\section{(c)}

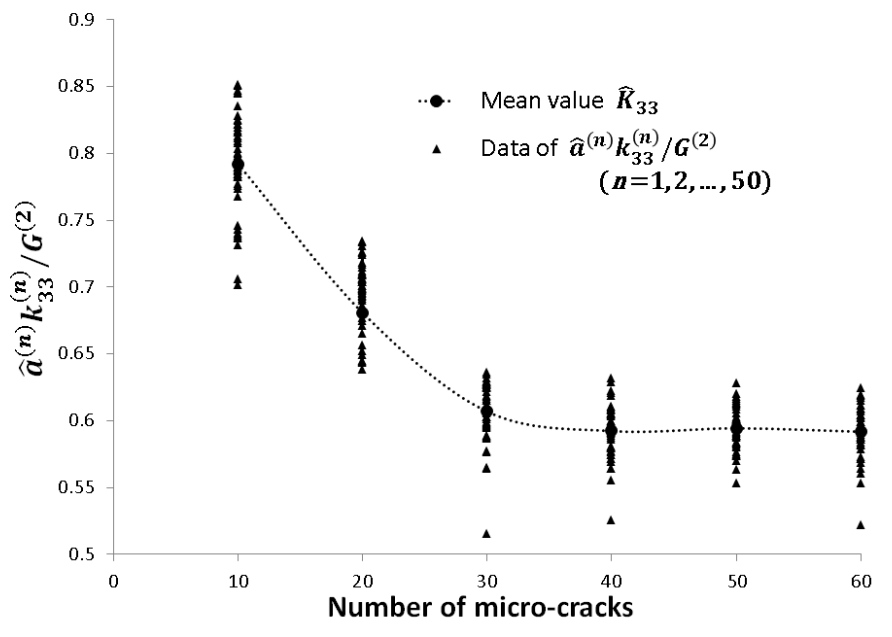

Figure 4.7: Scatter plots and mean values of the non-dimensionalized effective stiffness against $M$. 
degree of freedom.

\subsubsection{Influence of the Micro-crack Length Distribution}

The influence of the micro-crack length distribution on the mean values $\widehat{K}_{11}$, $\widehat{K}_{22}$ and $\widehat{K}_{33}$ of the non-dimensionalized effective stiffness coefficients is investigated here. For fixed values of $\rho$ and $\widehat{a} / h$, a sample of 50 randomly generated micro-cracked interfaces is used for the statistical simulation of the interface. As suggested by the analysis in Subsection 4.6.1, the number of micro-cracks over a period interval of the interface is taken to be 40 . For each interface, the micro-crack length follows the $\chi^{2}\left(m_{0}\right)$ distribution. To examine how the mean values $\widehat{K}_{11}, \widehat{K}_{22}$ and $\widehat{K}_{33}$ may be affected by different values of $m_{0}$ (that is, by different micro-crack length distributions), the micro-cracks are positioned in such a way that the crack-tip gap $g$ between two adjacent micro-cracks is a constant given by $g=2 \widehat{a}(1-\rho) / \rho$. For inplane deformations, the materials in the thin layer and the half-space are selected to be graphite-epoxy and E-glass-expoxy respectively.

For $\rho=0.5$, the mean values $\widehat{K}_{11}, \widehat{K}_{22}$ (for inplane deformations) and $\widehat{K}_{33}$ (for antiplane deformations) for selected values of $G^{(1)} / G^{(2)}$ are plotted against $\widehat{a} / h$ respectively in Figures 4.8 and 4.9 for cases where the $\chi^{2}(5), \chi^{2}(10)$ and $\chi^{2}(25)$ distribution are used for generating the lengths of the micro-cracks. Plots of the corresponding non-dimensionalized effective stiffness coefficients predicted by the three-phase model are also given in Figures 4.8 and 4.9 . It is obvious from Figures 4.8 and 4.9 that the mean values of the effective stiffness coefficients are influenced by the random micro-crack length distribution. It is also observed that the values of $\widehat{K}_{11}, \widehat{K}_{22}$ and $\widehat{K}_{33}$ become closer to the corresponding values predicted by the three-phase model, as the micro-crack length distribution becomes more normal-like, that is, the degree of freedom 
(a)

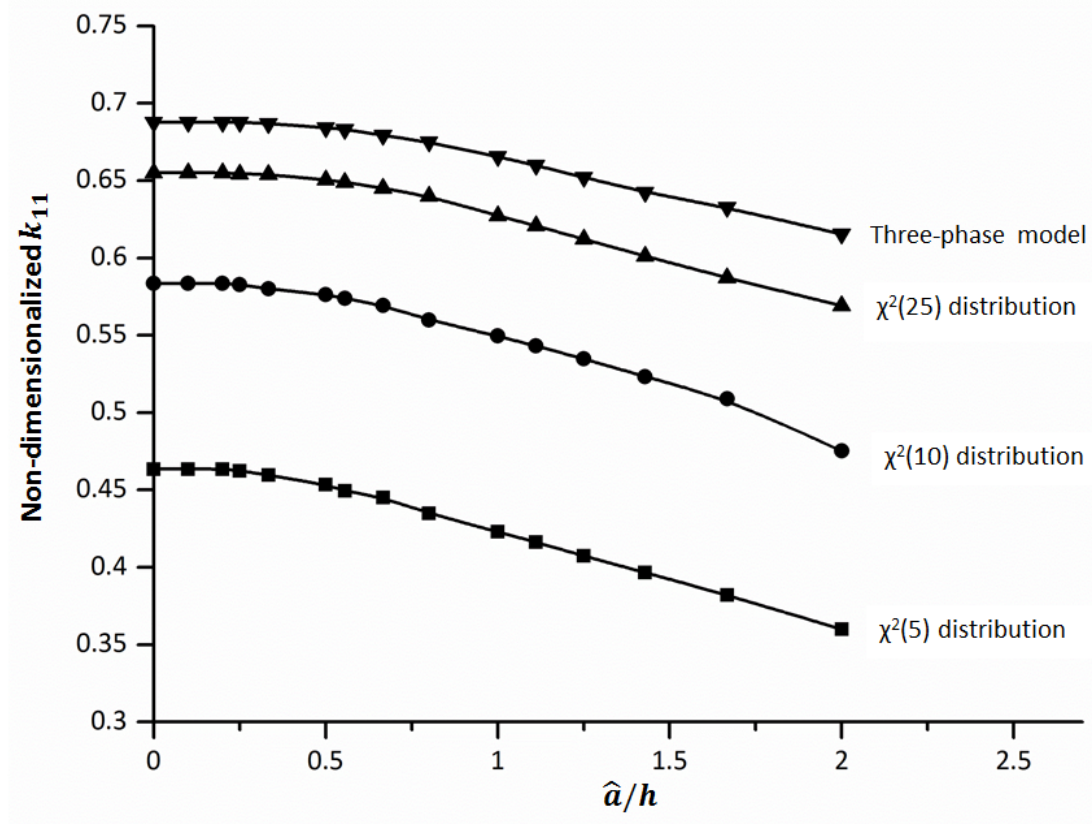

(b)

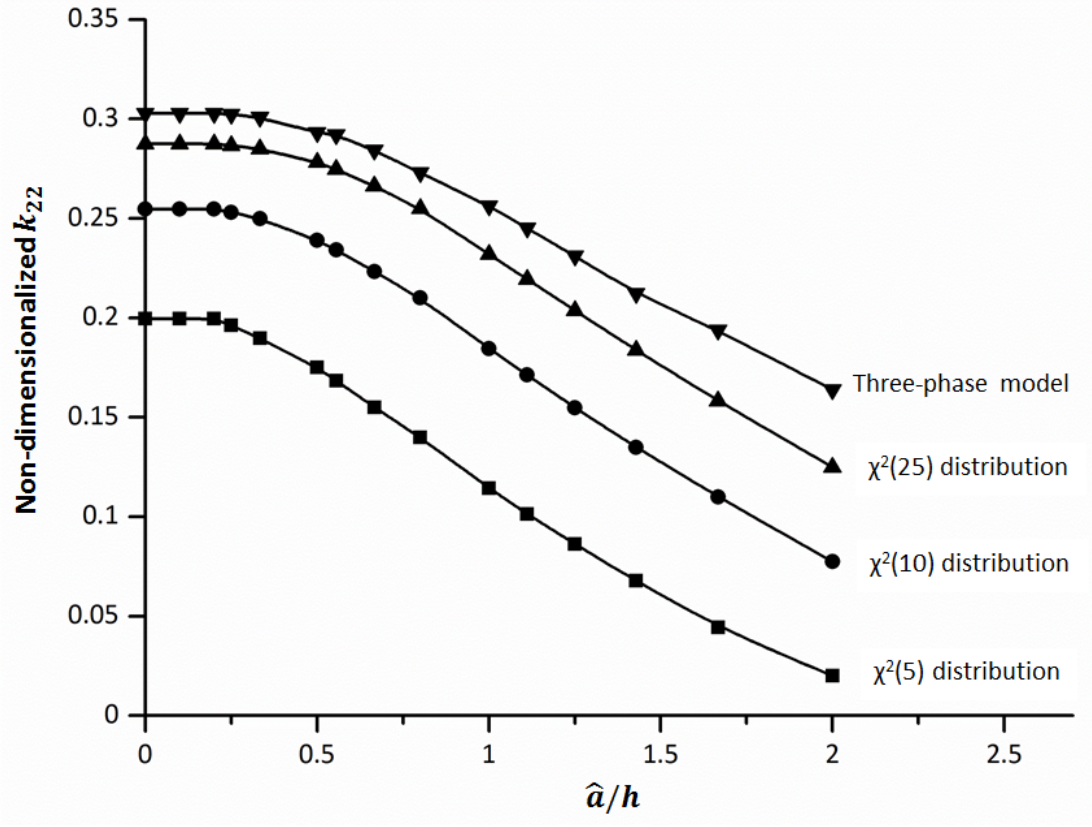

Figure 4.8: Plots of $\widehat{K}_{11}$ and $\widehat{K}_{22}$ against $\widehat{a} / h$ for $\rho=0.5$ and cases where the micro-crack length follows the $\chi^{2}(5), \chi^{2}(10)$ and $\chi^{2}(25)$ distributions. Also given are plots of the corresponding non-dimensionalized stiffness coefficients computed by using the three-phase model. 
(a) $G^{(1)} / G^{(2)}=0.5$

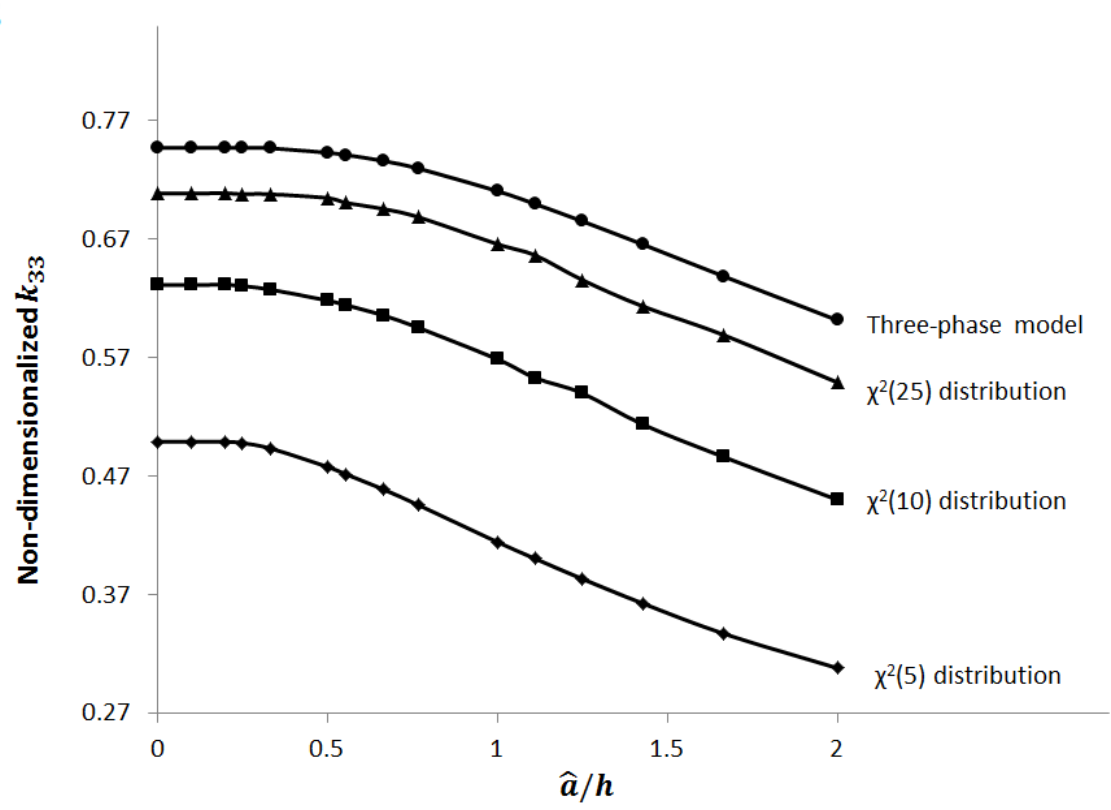

(b) $G^{(1)} / G^{(2)}=2$

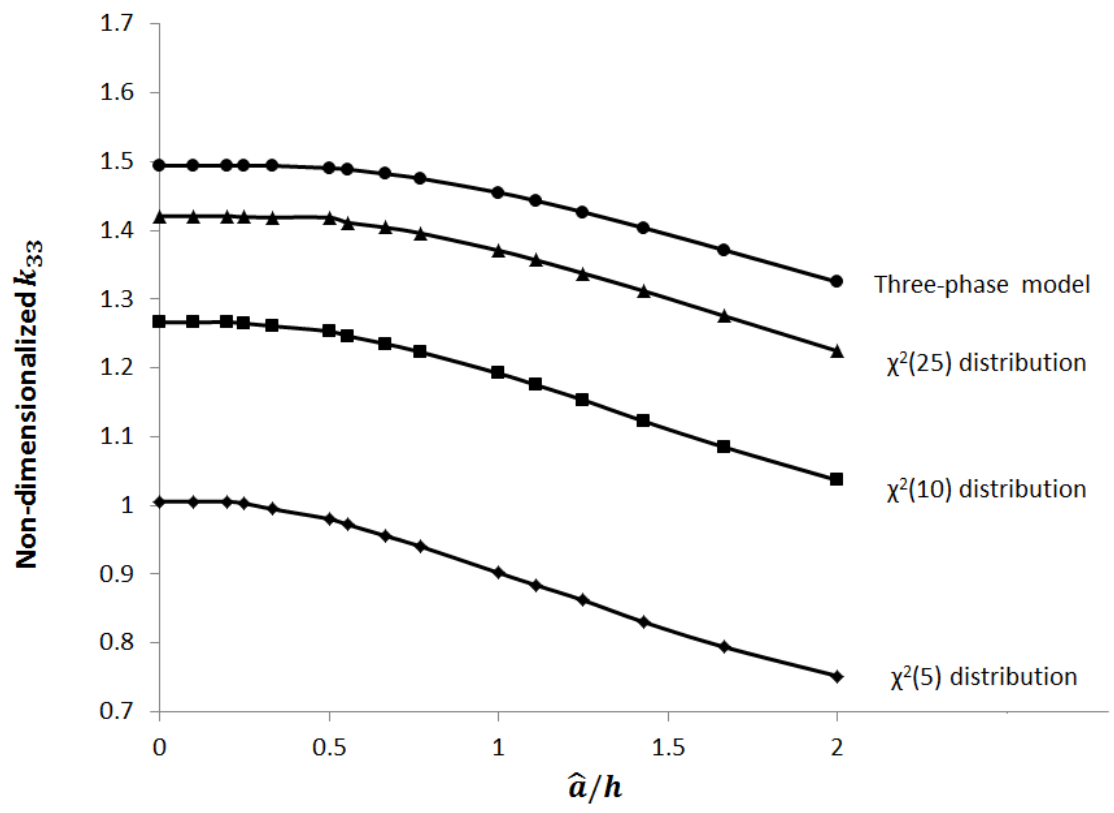

Figure 4.9: Plots of $\widehat{K}_{33}$ against $\widehat{a} / h$ for $\rho=0.5$, selected values of $G^{(1)} / G^{(2)}$ and cases where the micro-crack length follows the $\chi^{2}(5), \chi^{2}(10)$ and $\chi^{2}(25)$ distributions. Also given are plots of the corresponding non-dimensionalized stiffness coefficients computed by using the three-phase model. 
$k$ of the $\chi^{2}$ distributions increases. These observations in Figures 4.8 and 4.9 are similar to the corresponding ones in Figure 3.7. Refer to Subsection 3.7.2 for detailed explanations for the observations.

\subsubsection{Case Studies Using Specific Orthotropic Materials for Inplane Deformations}

For inplane deformations, the micromechanical-statistical model is used here to simulate the micro-cracked interface between a selected pair of dissimilar orthotropic materials.

As before, for selected values of $\rho$ and $\widehat{a} / h, 50$ micro-cracked interfaces are randomly generated for the statistical simulations. For each interface, 40 micro-cracks are randomly chosen with their lengths following the $\chi^{2}(5)$ distribution. The micro-cracks are positioned randomly over a period interval of the interface.

The elastic moduli (in GPa) for boron-epoxy as given in Table 3.1 are used as the material constants for the elastic half-space. Three different orthotropic materials of differing strength, namely carbon-epoxy, graphite-epoxy and martensite, with elastic moduli as given in Table 3.1, are selected in turn to be the material in the thin layer.

For $\rho=0.5$, the mean values $\widehat{K}_{11}$ and $\widehat{K}_{22}$ against $\widehat{a} / h$ are plotted in Figure 4.10 for the cases where carbon-epoxy, graphite-epoxy and martensite are used as the material in the thin layer. For a fixed $\widehat{a} / h$, the interface for the case where the thin layer is occupied by martensite has the largest effective stiffness coefficients $\widehat{K}_{11}$ and $\widehat{K}_{22}$, while the values of $\widehat{K}_{11}$ and $\widehat{K}_{22}$ are the smallest for the case where the thin layer is carbon-epoxy. Thus, the effective stiffness coefficients of the interface appear to be larger in magnitude if the material of the thin layer has larger elastic moduli. This observation is consistent with 
(a)

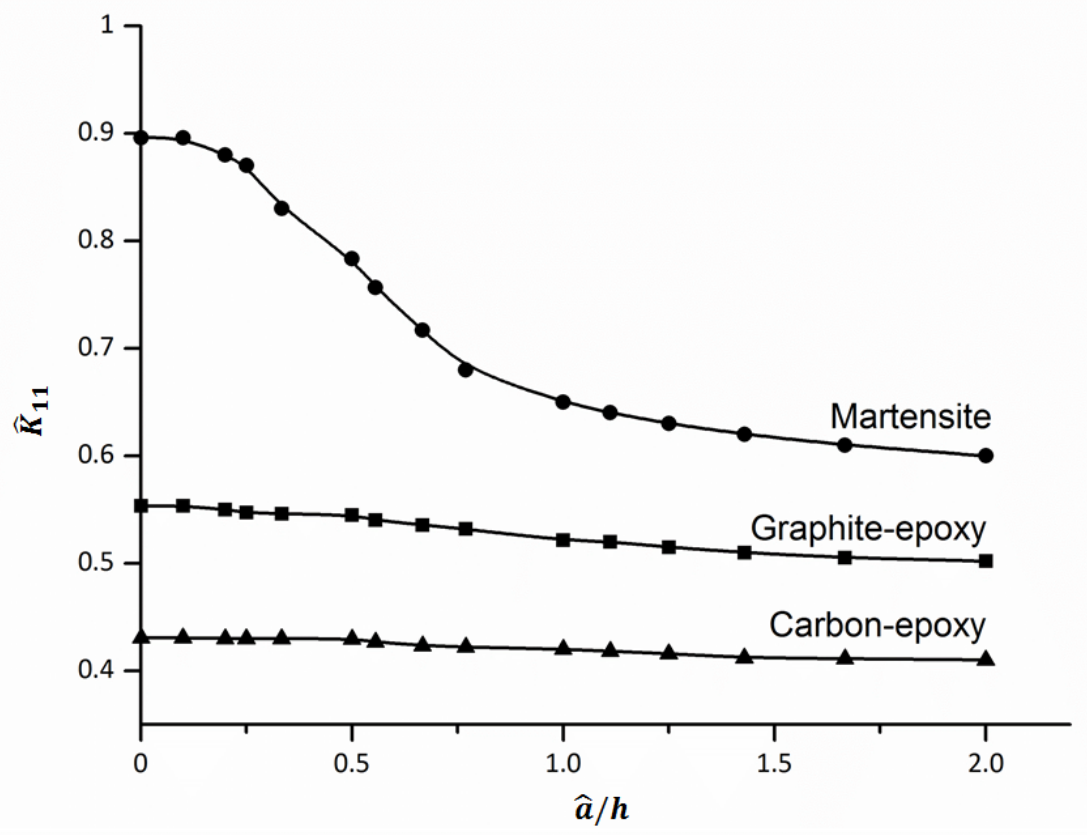

(b)

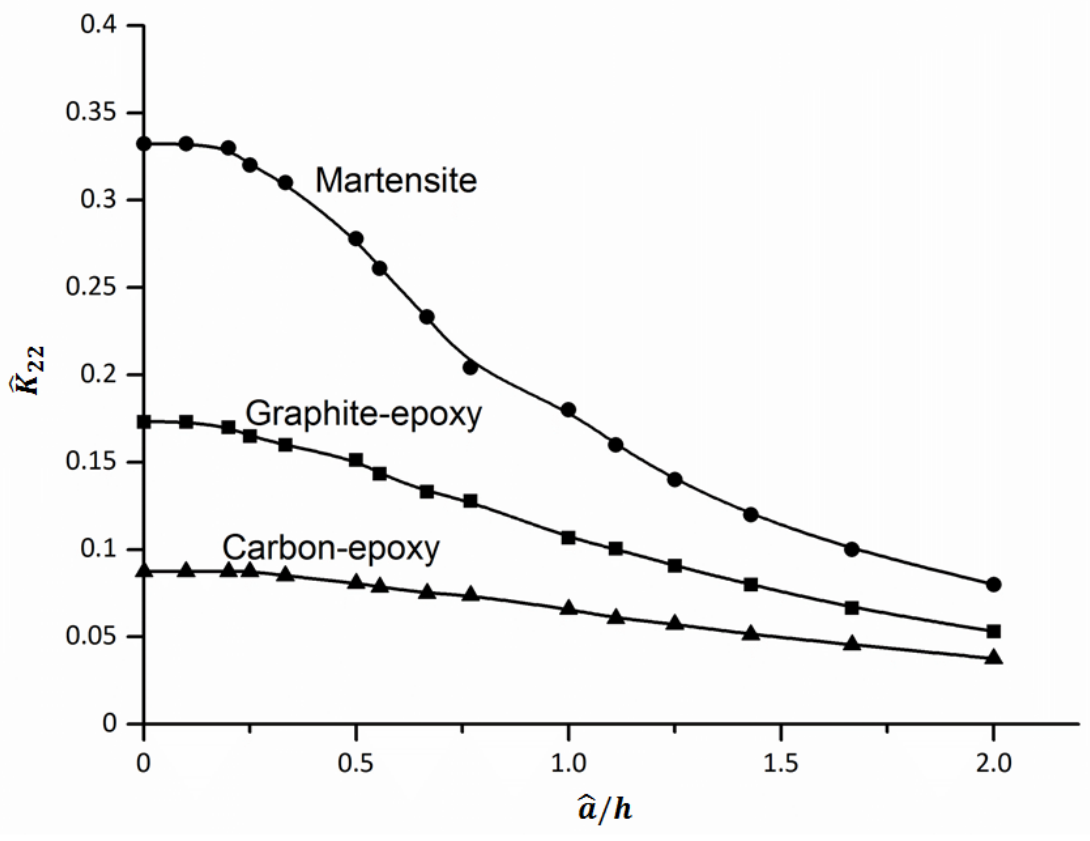

Figure 4.10: Plots of $\widehat{K}_{11}$ and $\widehat{K}_{22}$ against $\widehat{a} / h$ for selected thin layer materials. 
our intuition that coating a layer of "stronger" material on the half-space may reduce the magnitudes of the displacement jumps over the micro-cracks to give rise to larger effective stiffness coefficients. Note that the effective stiffness coefficients are calculated using the average displacement jumps over the micro-cracks.

Each of the plots in Figure 4.10 shows that the mean values $\widehat{K}_{11}$ and $\widehat{K}_{22}$ decrease as $\widehat{a} / h$ increases. As explained in Section 4.5 , as $\widehat{a} / h$ increases, that is, as the width of the layer becomes smaller compared to the average length of the micro-cracks, the edge of the layer has the effect of increasing the magnitudes of the displacement jumps across the micro-cracks, thereby reducing the effective stiffness coefficients of the interface.

\subsubsection{Isotropic Bimaterial Subject to Inplane Deforma- tions}

For inplane deformations, the case where the elastic layer and the elastic half-space are both isotropic is considered here. The hypersingular boundary integro-differential equations for the micromechanical-statistical model may be recovered for isotropic materials as explained in Subsection 3.7.5.

For isotropic materials, the hypersingular boundary integro-differential equations in (4.24) and (4.25) for the micromechanical-statistical model reduce to

$$
\begin{aligned}
& \frac{1}{2 \pi} f_{0}^{L} \frac{1}{\gamma} u_{r}^{(\mathrm{imp})}\left(x_{1}, h\right) \widetilde{G}_{r i}\left[\frac{1}{\left(x_{1}-\xi_{1}\right)^{2}}+\Theta\left(x_{1}, \xi_{1}\right)\right] d x_{1} \\
& +\frac{1}{2 \pi} \int_{0}^{L} \frac{1}{\gamma} u_{r}^{(\mathrm{imp})}\left(x_{1}, h\right) \operatorname{Re}\left\{\widetilde{H}_{r i} \Omega\left(x_{1}, \xi_{1}, 2 i h\right)\right\} d x_{1} \\
& -\frac{1}{2 \pi} \sum_{k=1}^{M} \int_{a^{(k)}}^{b^{(k)}} \frac{1}{\gamma} \Delta u_{r}^{(\mathrm{imp})}\left(x_{1}\right) \operatorname{Re}\left\{\widetilde{G}_{r i} \Omega\left(x_{1}, \xi_{1},-i h\right)\right\} d x_{1}
\end{aligned}
$$




$$
\begin{aligned}
& -\frac{1}{2 \pi} \sum_{k=1}^{M} \int_{a^{(k)}}^{b^{(k)}} \frac{1}{\gamma} \Delta u_{r}^{(\mathrm{imp})}\left(x_{1}\right) \operatorname{Re}\left\{\widetilde{H}_{r i} \Omega\left(x_{1}, \xi_{1}, i h\right)\right\} d x_{1} \\
= & 0 \text { for } 0<\xi_{1}<L,
\end{aligned}
$$

and

$$
\begin{aligned}
& \quad-\frac{1}{2 \pi} \int_{0}^{L} \frac{1}{\gamma} u_{r}^{(\mathrm{imp})}\left(x_{1}, h\right) \operatorname{Re}\left\{\Omega\left(x_{1}, \xi_{1}, i h\right)\left(\widetilde{G}_{r i}+\widetilde{H}_{r i}\right)\right\} d x_{1} \\
& +\frac{1}{2 \pi}\left[\int_{a^{(k)}}^{b^{(k)}} \frac{\Delta u_{r}^{(\mathrm{imp})}\left(x_{1}\right) \widetilde{W}_{r i}}{\gamma\left(x_{1}-\xi_{1}\right)^{2}} d x_{1}\right. \\
& +\sum_{\substack{n=1 \\
n \neq k}}^{M} \int_{a^{(n)}}^{b^{(n)}} \frac{\Delta u_{r}^{(\mathrm{imp})}\left(x_{1}\right) \widetilde{W}_{r i}}{\gamma\left(x_{1}-\xi_{1}\right)^{2}} d x_{1} \\
& \left.+\sum_{n=1}^{M} \int_{a^{(n)}}^{b^{(n)}} \frac{1}{\gamma} \Delta u_{r}^{(\mathrm{imp})}\left(x_{1}\right) \widetilde{W}_{r i} \Theta\left(x_{1}, \xi_{1}\right) d x_{1}-\frac{\pi \widetilde{V}_{r i}}{\gamma} \frac{d\left(\Delta u_{r}^{(\mathrm{imp})}\left(\xi_{1}\right)\right)}{d \xi_{1}}\right] \\
& =\delta_{i j} \sigma_{j 2}^{(\mathrm{ext})}\left(\xi_{1}, 0^{+}\right) \text {for } a^{(k)}<\xi_{1}<b^{(k)}(k=1,2, \ldots, M),
\end{aligned}
$$

where $\widetilde{G}_{r i}$ and $\widetilde{H}_{r i}$ are given by

$$
\begin{aligned}
& {\left[\widetilde{G}_{r i}\right]=\left[\begin{array}{cc}
-\frac{4}{1+\alpha} & 0 \\
0 & -\frac{4}{1+\alpha}
\end{array}\right],} \\
& {\left[\widetilde{H}_{r i}\right]=\left[\begin{array}{cc}
-\frac{4}{1-\beta^{2}}+\frac{4}{1+\alpha} & \frac{4 \beta}{1-\beta^{2}} i \\
-\frac{4 \beta}{1-\beta^{2}} i & -\frac{4}{1-\beta^{2}}+\frac{4}{1+\alpha}
\end{array}\right],}
\end{aligned}
$$

with $\alpha$ given by

$$
\alpha=\frac{1}{\gamma}\left(\frac{\lambda^{(1)}+2 \mu^{(1)}}{\mu^{(1)}\left(\lambda^{(1)}+\mu^{(1)}\right)}-\frac{\lambda^{(2)}+2 \mu^{(2)}}{\mu^{(2)}\left(\lambda^{(2)}+\mu^{(2)}\right)}\right),
$$

the constant $\gamma$ is given by (3.80), $\widetilde{W}_{r i}$ and $\widetilde{V}_{r i}$ are defined in (3.81) and $\beta$ is given by (3.82). Note that the non-dimensionalized constants $\alpha$ and $\beta$ are known as the Dundurs' parameters in the literature (see, for example, [71] and [72]). 


\section{Micromechanical Models for Weak Interfaces}

As in [72], the Dundurs' parameters $\alpha$ and $\beta$ and the parameter $\gamma$ in (4.32), (3.82) and (3.80) may be rewritten in terms of the Young's moduli $E^{(1)}$ and $E^{(2)}$ and the Poisson's ratios $\nu^{(1)}$ and $\nu^{(2)}$ (of the thin elastic layer and the elastic half-space) as

$$
\begin{aligned}
& \alpha=\frac{\frac{E^{(2)}}{E^{(1)}}-\frac{\left(1-\nu^{(2)}\right)\left(1+\nu^{(2)}\right)}{\left(1-\nu^{(1)}\right)\left(1+\nu^{(1)}\right)}}{\frac{E^{(2)}}{E^{(1)}}+\frac{\left(1-\nu^{(2)}\right)\left(1+\nu^{(2)}\right)}{\left(1-\nu^{(1)}\right)\left(1+\nu^{(1)}\right)}} \\
& \beta=\frac{1}{2} \frac{\frac{E^{(2)}}{E^{(1)}}\left(1-2 \nu^{(1)}\right)\left(1+\nu^{(1)}\right)-\left(1-2 \nu^{(2)}\right)\left(1+\nu^{(2)}\right)}{\frac{E^{(2)}}{E^{(1)}}\left(1-\nu^{(1)}\right)\left(1+\nu^{(1)}\right)+\left(1-\nu^{(2)}\right)\left(1+\nu^{(2)}\right)}, \\
& \gamma=\frac{4}{E^{(2)}}\left[\frac{E^{(2)}}{E^{(1)}}\left(1-\nu^{(1)}\right)\left(1+\nu^{(1)}\right)+\left(1-\nu^{(2)}\right)\left(1+\nu^{(2)}\right)\right] .
\end{aligned}
$$

For a particular case study, we take $\nu^{(1)}=\nu^{(2)}=\nu$ and examine how $E^{(1)} / E^{(2)}$ and $\nu$ may affect the effective stiffness coefficients $k_{11}$ and $k_{22}$. For selected $E^{(1)} / E^{(2)}, \nu, \rho$ and $\widehat{a} / h$, a sample of 50 interfaces are randomly generated. Each interface contains 40 micro-cracks that are randomly positioned over a period interval of the interface. The micro-crack length follows the $\chi^{2}(5)$ distribution. For such cases, the non-dimensionalized effective stiffness coefficients for inplane deformations in (4.29) for the $n$-th interface are written as

$$
\begin{aligned}
& K_{11}^{(n)}=\widehat{a}^{(n)} k_{11}^{(n)} / E^{(2)}, \\
& K_{22}^{(n)}=\widehat{a}^{(n)} k_{22}^{(n)} / E^{(2)} .
\end{aligned}
$$

The mean values $\widehat{K}_{11}$ and $\widehat{K}_{22}$ of the non-dimensionalized effective stiffness coefficients in (4.34) of the 50 randomly generated interfaces are calculated by using (3.47). 


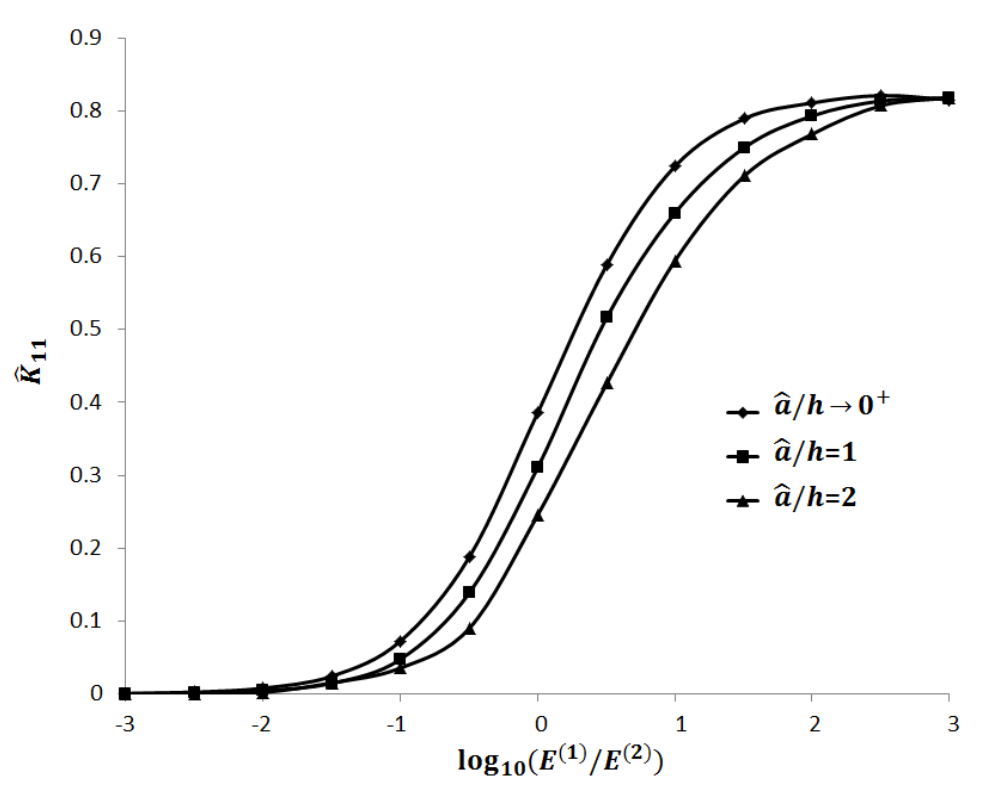

Figure 4.11: Plots of $\widehat{K}_{11}$ for isotropic bimaterial against $\log _{10}\left(E^{(1)} / E^{(2)}\right)$ for selected values of $\widehat{a} / h, \rho=0.5$ and $\nu=0.25$.

For $\rho=0.5$ and $\nu=0.25$, plots of the non-dimensionalized effective stiffness coefficients $\widehat{K}_{11}$ against $\log _{10}\left(E^{(1)} / E^{(2)}\right)$ for selected values of $\widehat{a} / h$ are given in Figure 4.11. Note that the values of $\widehat{K}_{22}$ are observed to be very close to the values of $\widehat{K}_{11}$ for isotropic bimaterials. For moderate values of $E^{(1)} / E^{(2)}$ within the range $-0.5<\log _{10}\left(E^{(1)} / E^{(2)}\right)<2$, the plots for various $\widehat{a} / h$ are visually distinguishable and it is obvious that $\widehat{K}_{11}$ increases with the increasing $\log _{10}\left(E^{(1)} / E^{(2)}\right)$ for a fixed $\widehat{a} / h$. For $E^{(1)} / E^{(2)}$ larger than 100 , all the plots of $\widehat{K}_{11}$ in Figure 4.11 tend to the same constant, approximately 0.81 , as $E^{(1)} / E^{(2)}$ increases. It appears that the effect of the edge of the thin layer becomes negligible if the thin layer can be made sufficiently strong compared to the elastic half-space. The values of $\widehat{K}_{11}$ tend to zero as $E^{(1)} / E^{(2)}$ tends to zero for the selected values of $\widehat{a} / h$.

For $\rho=0.5$ and $\widehat{a} / h=1$, plots of the non-dimensionalized effective stiff- 


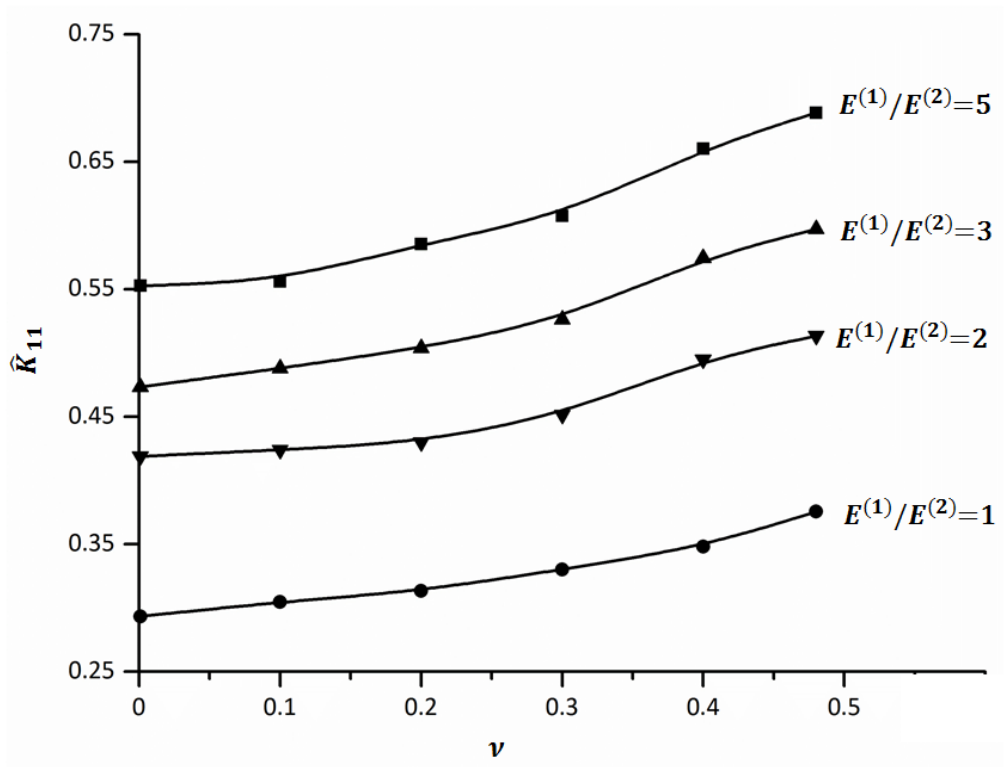

Figure 4.12: Plots of $\widehat{K}_{11}$ for isotropic bimaterial against $\nu$ for selected values of $E^{(1)} / E^{(2)}, \rho=0.5$ and $\widehat{a} / h=1$.

ness coefficients $\widehat{K}_{11}$ against $\nu$ are given in Figure 4.12 for selected values of $E^{(1)} / E^{(2)}$. For a fixed $E^{(1)} / E^{(2)}$, the values of $\widehat{K}_{11}$ increase as $\nu$ increases from 0 to 0.5 . It appears that the effective stiffness coefficients of the interface increase as the isotropic materials in the elastic layer and the elastic half-space becomes more incompressible.

\subsubsection{Parametric Studies for Antiplane Deformations}

To conduct some parametric investigations on the effective stiffness of the micro-cracked interface under antiplane deformations, 50 interfaces are randomly generated for selected $\widehat{a} / h, G^{(1)} / G^{(2)}$ and $\rho$. For each interface, the $\chi^{2}(5)$ distribution is used to generate the lengths of 40 micro-cracks which are randomly positioned on a period length of the interface. For the $n$-th interface, the non-dimensionalized effective stiffness for antiplane deformations is given 
in (4.29). The mean value $\widehat{K}_{33}$ of the non-dimensionalized effective stiffness calculated from the 50 interfaces is estimated by using (3.46).

Figure 4.13 plots the mean value $\widehat{K}_{33}$ of the non-dimensionalized effective stiffness against the damage ratio $\rho$ for selected values of $\widehat{a} / h$ and $G^{(1)} / G^{(2)}$. Three obvious trends are observed in Figure 4.13. Firstly, for fixed values of $\widehat{a} / h$ and $G^{(1)} / G^{(2)}$, the non-dimensionalized effective stiffness of the interface decreases with increasing damage ratio $\rho$. This is not surprising as the microcracks occupy a larger portion of the interface, have smaller crack-tip gaps and tend to be less stable if the damage ratio $\rho$ is larger. Secondly, for fixed values of $\rho$ and $G^{(1)} / G^{(2)}$, the non-dimensionalized effective stiffness decreases as $\widehat{a} / h$ increases. This is as expected as it is known that the external plane boundary of the thin layer has the effect of making the micro-cracks less stable. Thirdly, for fixed values of $\rho$ and $\widehat{a} / h$, the non-dimensionalized effective stiffness increases with increasing $G^{(1)} / G^{(2)}$. This may be explained as follows - coating the elastic half-space with a layer of stronger material has the overall effect of strengthening the half-space, thereby causing the micro-cracks to be more stable.

The non-dimensionalized effective stiffness is plotted against $\widehat{a} / h$ in Figure 4.14 for selected values of $\rho$ and $G^{(1)} / G^{(2)}$. For the values of $\rho$ and $G^{(1)} / G^{(2)}$ considered, the effective stiffness decreases with increasing $\widehat{a} / h$ for $\widehat{a} / h>1$, that is, if the width of the thin layer is less than the average half length of the micro-cracks. For $\widehat{a} / h<1$, the effective stiffness increases slowly as $\widehat{a} / h$ decreases and remains constant to within at least two significant figures for $\widehat{a} / h$ less than 0.25 . Further simulations show that the effective stiffness does not change significantly for $\widehat{a} / h=0.1,0.01$ and 0.001 . At least for $0.1 \leq G^{(1)} / G^{(2)} \leq 10$ and $0.1 \leq \rho \leq 0.9$, the analysis here seems to suggest that the external plane boundary of the thin layer has hardly any effect in 
(a) $G^{(1)} / G^{(2)}=10$

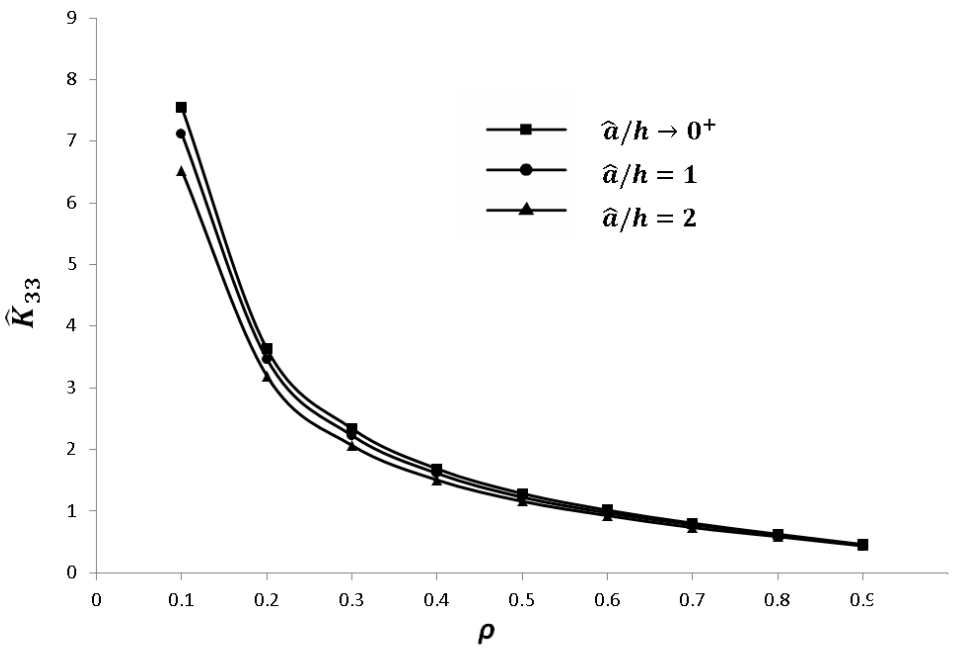

(b) $G^{(1)} / G^{(2)}=1$

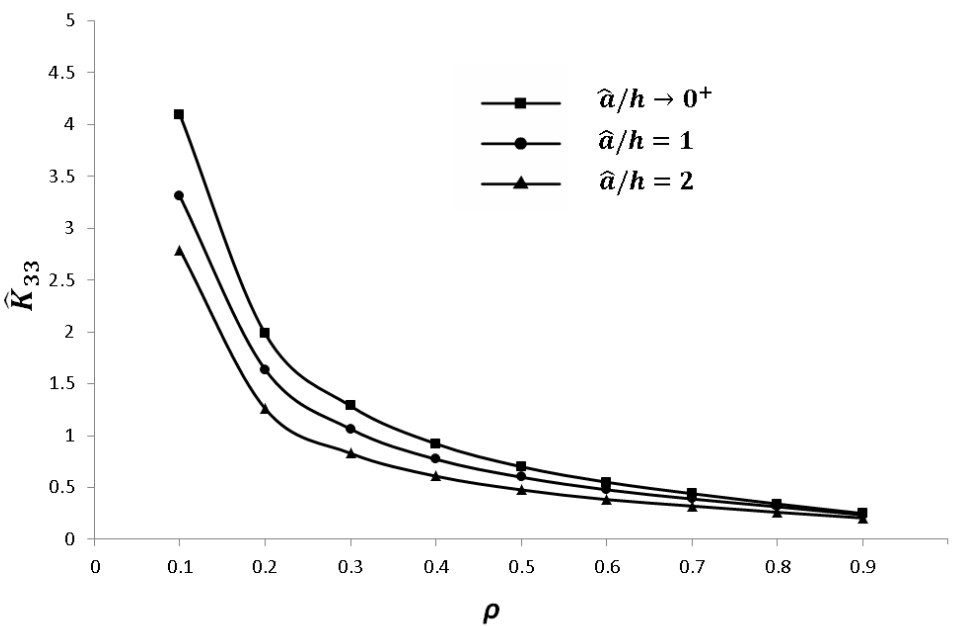

(c) $G^{(1)} / G^{(2)}=0.1$

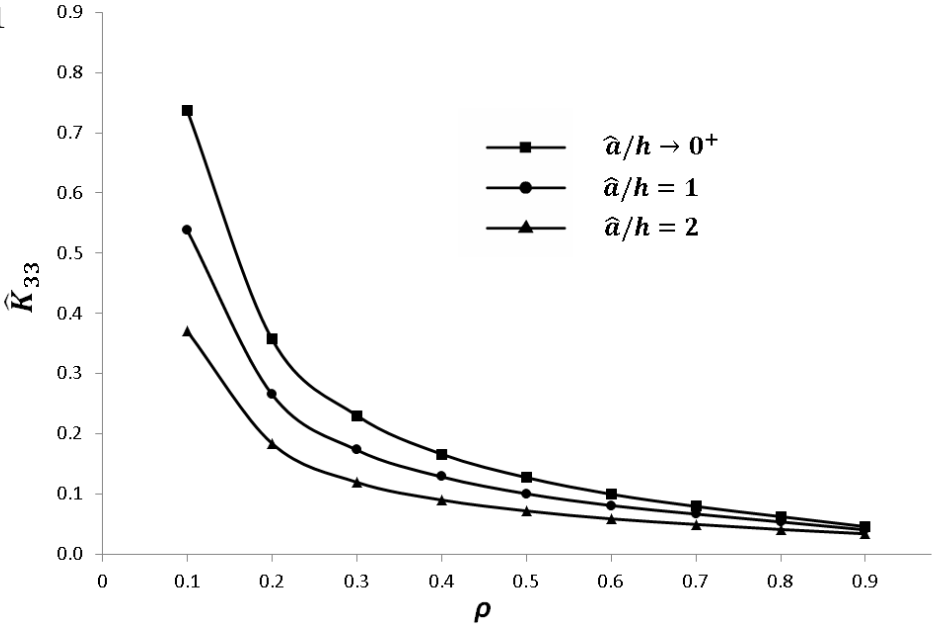

Figure 4.13: Plots of $\widehat{K}_{33}$ against $\rho$ for selected values of $\widehat{a} / h$ and $G^{(1)} / G^{(2)}$. 
(a) $\rho=0.1$

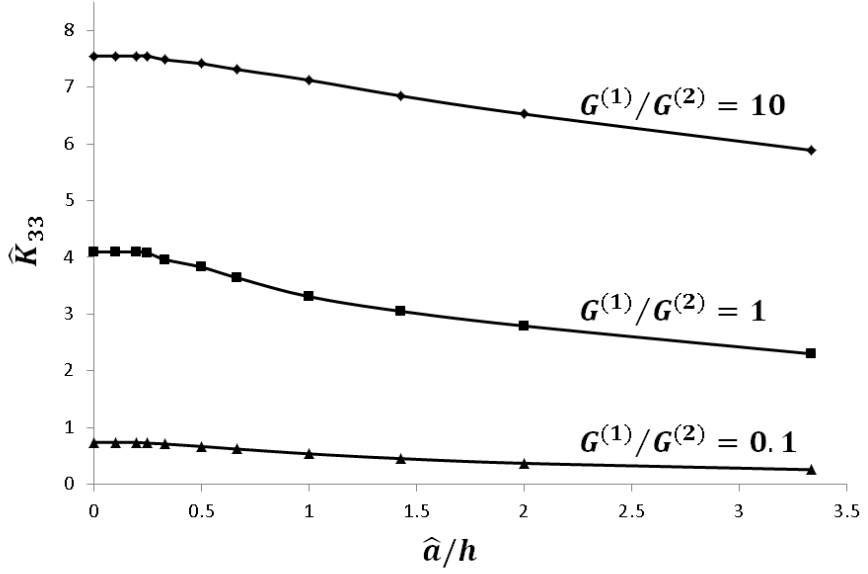

(b) $\rho=0.5$

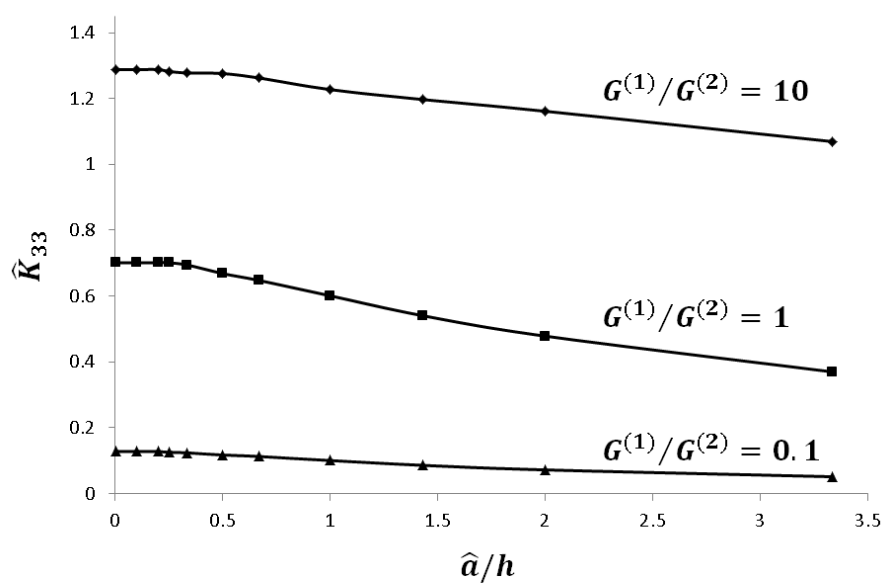

(c) $\rho=0.9$

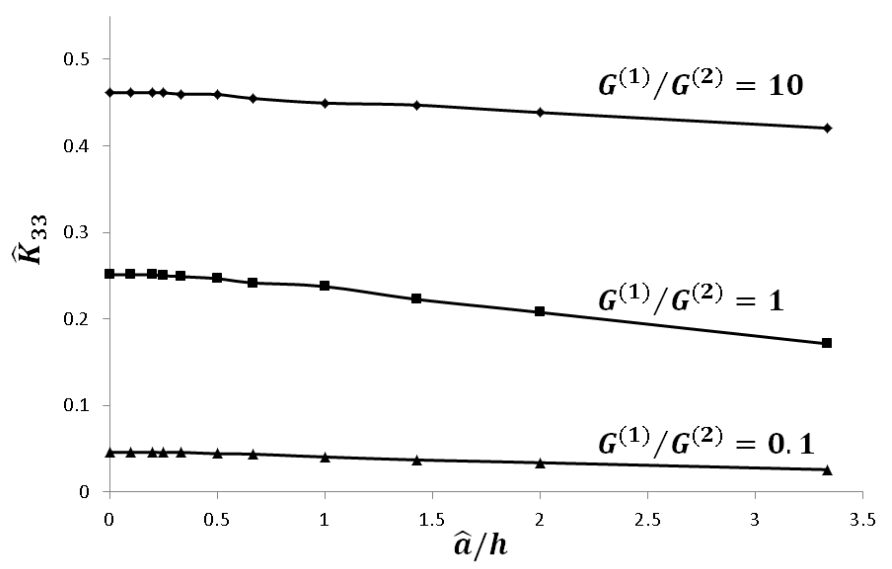

Figure 4.14: Plots of $\widehat{K}_{33}$ against $\widehat{a} / h$ for selected values of $G^{(1)} / G^{(2)}$ and $\rho$. 
influencing the effective stiffness of the interface if the width of the thin layer is at least four times larger than the average half length of the micro-cracks.

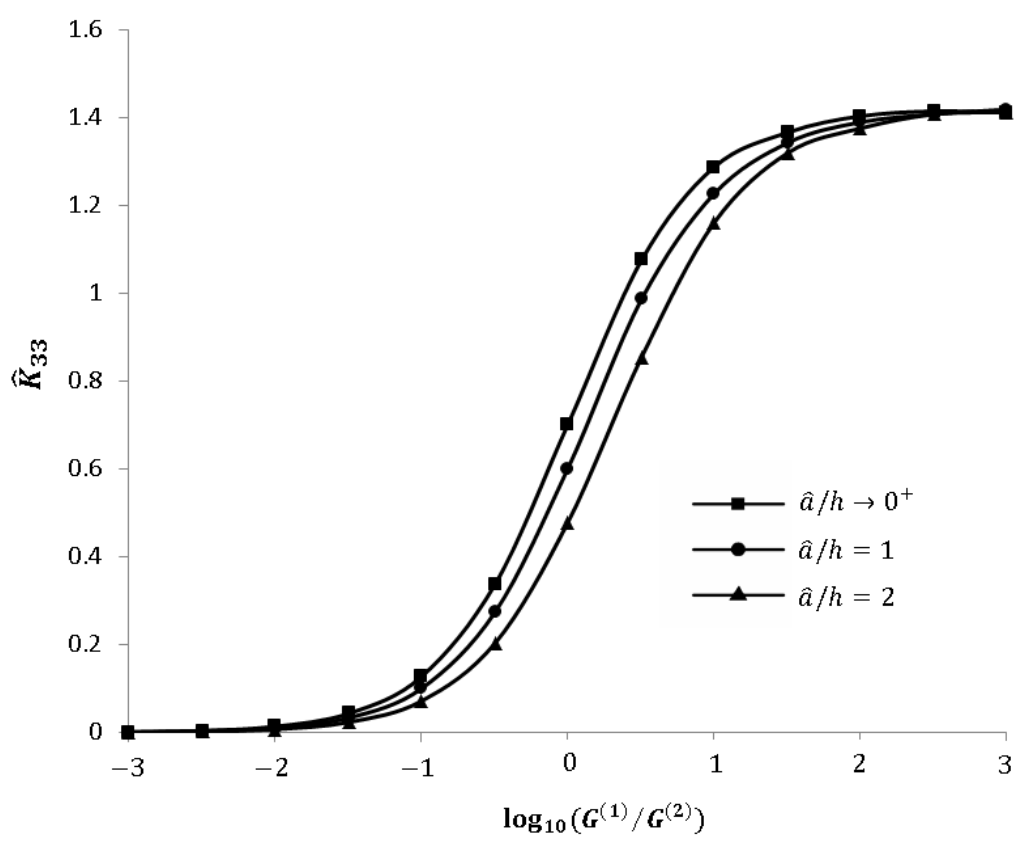

Figure 4.15: Plots of $\widehat{K}_{33}$ against $\log _{10}\left(G^{(1)} / G^{(2)}\right)$ for $\rho=0.5$ and selected values of $\widehat{a} / h$.

To examine the effect of varying $G^{(1)} / G^{(2)}$ on the effective stiffness of the interface, the mean value $\widehat{K}_{33}$ of the non-dimensionalized effective stiffness is plotted against $\log _{10}\left(G^{(1)} / G^{(2)}\right)$ in Figure 4.15 for $\rho=0.5$ and selected values of $\widehat{a} / h$. For moderate values of $G^{(1)} / G^{(2)}$ within the range where $-1.5<\log _{10}\left(G^{(1)} / G^{(2)}\right)<2$, the plots for the different values of $\widehat{a} / h$ are visually distinguishable. As pointed out earlier, for a fixed $G^{(1)} / G^{(2)}$, the nondimensionalized effective stiffness is larger for smaller $\widehat{a} / h$. For $G^{(1)} / G^{(2)}>100$, the non-dimensionalized effective stiffness increases very slowly as $G^{(1)} / G^{(2)}$ increases. At $G^{(1)} / G^{(2)}=1000$, it converges to the same constant value of 1.41 (to within at least two significant figures) for all the three values of $\widehat{a} / h$ con- 
sidered here. Thus, it appears that the external plane boundary of the thin layer has negligible effect on the effective stiffness if the shear modulus $G^{(1)}$ of the thin layer is very much larger than the shear modulus $G^{(2)}$ of the halfspace. As $G^{(1)} / G^{(2)} \rightarrow 0^{+}$, the non-dimensionalized effective stiffness tends to zero no matter what $\widehat{a} / h$ is, that is, if the thin layer is extremely soft relative to the half-space, the effective stiffness of the interface may be very small in magnitude.

\subsection{Summary}

Micromechanical models are formulated here for analyzing a micro-cracked interface between a thin orthotropic layer and an orthotropic half-space under antiplane and inplane deformations. The boundary integral equations of elasticity are used with the Green's functions for perfect interfaces to derive hypersingular boundary integro-differential equations for the micromechanical models.

As a check, the effective stiffness coefficients of the interface estimated by the three-phase model are compared with those calculated from the periodic model involving evenly distributed micro-cracks of equal length. As expected, the values of the effective stiffness coefficients given by the two models are in close agreement, especially when the damage ratio of the interface is not too close to unity.

Statistical simulations conducted on the micro-cracked interface suggest that at least 40 micro-cracks are required in the micromechanical-statistical model to homogenize the effective stiffness coefficients of the interface. Furthermore, the simulations show that the effective stiffness coefficients are significantly influenced by the degree of freedom of the $\chi^{2}$ distribution used to 
generate the lengths of the micro-cracks. As the degree of freedom increases, that is, as the variation of the micro-crack length compared to the average micro-crack length becomes smaller, the effective stiffness coefficients are closer to those predicted by the three-phase model. The three-phase model overestimates the values of the effective stiffness coefficients.

For inplane deformations, two specific case studies involving particular materials in the layer and the half-space are conducted using the micromechanicalstatistical model.

In the first case study, the effective stiffness coefficients of the interface are calculated for three different particular orthotropic materials in the layer. As expected, the effective stiffness coefficients are larger when the orthotropic material in the layer is "stronger". For a given interface between a given pair of materials, the effective stiffness coefficients increase as the width of the thin layer increases.

In the second case study, the elastic materials in the thin layer and the halfspace are taken to be isotropic. For isotropic materials, the coefficients in the hypersingular integral and integro-differential equations for the micromechanicalstatistical model are expressed in terms of the Dundurs' parameters $\alpha$ and $\beta$. The coefficients are found to be independent of $\alpha$ if the isotropic elastic layer is replaced by an isotropic elastic half-space. Statistical simulations conducted for the case where the materials in the layer and the half-space have the same Poisson's ratio show that the effect of the thin edge of the layer on the effective stiffness coefficients of the interface may be made negligible by increasing the Young's modulus of the material in the layer. Furthermore, the effective stiffness coefficients of the interface are observed to increase as the materials in the layer and the half-space become more incompressible.

For antiplane deformations, the micromechanical-statistical model is used 
to conduct some parametric studies on the effects of varying the damage ratio of the interface, the width of the thin layer and the ratio of the shear modulus in the thin layer to the one in the half-space on the effective stiffness of the interface. For fixed values of the average half crack length over the width of the thin layer and the shear modulus ratio, the effective stiffness is a decreasing function of the damage ratio of the interface. For fixed values of the damage ratio and the shear modulus ratio, the effective stiffness increases with the increasing width of the thin layer but appears to tend to a constant as the width of the thin layer tends to infinity. For a fixed non-zero value of the average half crack length over the width of the thin layer, the effect of the external plane boundary on the effective stiffness is reduced as the shear modulus ratio increases to an extremely large value, that is, as the thin layer becomes much harder relative to the half-space. 



\section{Chapter 5}

\section{Interaction of a Pair of Parallel Weak Interfaces}

\subsection{Introduction}

The interaction of a pair of parallel micro-cracked interfaces in a trimaterial under antiplane and inplane deformations is considered in this chapter. The trimaterial is made of a thin orthotropic elastic layer sandwiched between two orthotropic elastic half-spaces. The micro-cracks on each interface are periodically distributed and they have possibly different lengths and positions over a period interval of the interface. The width of the thin layer is comparable to the lengths of the micro-cracks on the interfaces.

To formulate mathematically the problem under consideration here, the trimaterial is divided into two subdomains along an artificial plane boundary in the middle of the thin layer. Each of the subdomains may be regarded as a bimaterial consisting of a thin elastic layer and an elastic half-space. Guided by the analysis in Chapter 4, we use the boundary integral equations together with the perfect Green's functions for bimaterials to derive a set of hypersingular boundary integro-differential equations for each of the two subdomains. The unknown functions in the hypersingular boundary integro-differential equa- 
tions are the displacement jumps across the micro-cracks on the two interfaces and the displacements and the normal stresses on the artificial plane boundary. For a complete formulation of the problem, the displacements and the normal stresses are required to be continuous on the artificial boundary. Once the hypersingular boundary integro-differential equations are solved together with the continuity conditions on the artificial boundary, the effective stiffness coefficients of the interfaces may be readily computed.

Some specific case studies are conducted to investigate the effects of the material constants of the thin layer and the two half-spaces, the width of the elastic layer and the densities of the micro-cracks on the effective stiffness coefficients of the two interfaces.

\subsection{A Pair of Micro-cracked Interfaces}

With reference to a Cartesian coordinate system $O x_{1} x_{2} x_{3}$, a thin elastic layer occupies the region $0<x_{2}<h$ and is sandwiched between two elastic halfspaces in the regions $x_{2}<0$ and $x_{2}>h$. The interfaces $x_{2}=0$ and $x_{2}=h$ between the layer and the half-spaces are microscopically damaged, containing interfacial micro-cracks. For convenience, the micro-cracked interfaces $x_{2}=0$ and $x_{2}=h$ are denoted by I and II respectively.

The trimaterial is assumed to undergo either an antiplane or inplane deformation independent of the $x_{3}$ coordinate. The governing partial differential equations for the trimaterial under antiplane or inplane deformation are given in Section 2.2 with the elastic constants for the orthotropic materials given by

$$
\begin{aligned}
& \left(C_{11}, C_{22}, C_{12}, C_{66}, C_{44}, C_{55}\right) \\
& = \begin{cases}\left(C_{11}^{(1)}, C_{22}^{(1)}, C_{12}^{(1)}, C_{66}^{(1)}, C_{44}^{(1)}, C_{55}^{(1)}\right) & \text { for } x_{2}>h, \\
\left(C_{11}^{(2)}, C_{22}^{(2)}, C_{12}^{(2)}, C_{66}^{(2)}, C_{44}^{(2)}, C_{55}^{(2)}\right) & \text { for } 0<x_{2}<h, \\
\left(C_{11}^{(3)}, C_{22}^{(3)}, C_{12}^{(3)}, C_{66}^{(3)}, C_{44}^{(3)}, C_{55}^{(3)}\right) & \text { for } x_{2}<0 .\end{cases}
\end{aligned}
$$


As in Figures 3.1 and 4.1, each of the interfaces here is modeled as containing periodical arrays of interfacial micro-cracks. For a simplified model, each interface is taken to contain $M$ arbitrarily positioned micro-cracks of possibly different lengths lying on a period interval of the interface of length $L$. On the part of interface I where $0<x_{1}<L$, the tips of a typical $m$-th micro-crack are given by $\left(a_{\mathrm{I}}^{(m)}, 0\right)$ and $\left(b_{\mathrm{I}}^{(m)}, 0\right)$, where $a_{\mathrm{I}}^{(m)}$ and $b_{\mathrm{I}}^{(m)}$ are constants such that $0<a_{\mathrm{I}}^{(1)}<b_{\mathrm{I}}^{(1)}<a_{\mathrm{I}}^{(2)}<b_{\mathrm{I}}^{(2)}<\cdots<a_{\mathrm{I}}^{(M)}<b_{\mathrm{I}}^{(M)}<L$. On the remaining parts of interface I, the interfacial micro-cracks lie at $a_{\mathrm{I}}^{(m)}+n L<x_{1}<b_{\mathrm{I}}^{(m)}+n L$, for $m=1,2, \cdots, M$ and $n= \pm 1, \pm 2, \cdots$. The part of interface II where $L_{0}<x_{1}<L_{0}+L$ contains $M$ micro-cracks with tips $\left(a_{\mathrm{II}}^{(m)}, 0\right)$ and $\left(b_{\mathrm{II}}^{(m)}, 0\right)$ (for $m=1,2, \cdots, M)$, where $L_{0}$ is a given positive number such that $0 \leq L_{0}<L$ and $a_{\mathrm{II}}^{(m)}$ and $b_{\mathrm{II}}^{(m)}$ are such that $L_{0}<a_{\mathrm{II}}^{(1)}<b_{\mathrm{II}}^{(1)}<a_{\mathrm{II}}^{(2)}<b_{\mathrm{II}}^{(2)}<\cdots<a_{\mathrm{II}}^{(M)}<$ $b_{\mathrm{II}}^{(M)}<L_{0}+L$. The micro-cracks on interface II outside $L_{0}<x_{1}<L_{0}+L$ lie in the regions $a_{\mathrm{II}}^{(m)}+n L<x_{1}<b_{\mathrm{II}}^{(m)}+n L, x_{2}=0$, for $m=1,2, \cdots, M$ and $n= \pm 1, \pm 2, \cdots$. Figure 5.1 gives a sketch of the geometry of the trimaterial for $M=3$. The uncracked parts of the interfaces are perfectly bonded.

The damage ratios of interfaces I and II are respectively defined by

$$
\rho_{\mathrm{I}}=\frac{1}{L} \sum_{m=1}^{M}\left(b_{\mathrm{I}}^{(m)}-a_{\mathrm{I}}^{(m)}\right) \text { and } \rho_{\mathrm{II}}=\frac{1}{L} \sum_{m=1}^{M}\left(b_{\mathrm{II}}^{(m)}-a_{\mathrm{II}}^{(m)}\right) .
$$

From (5.2), because of the assumption that the two interfaces have the same period length and the same number of micro-cracks over a period interval, the damage ratios can be shown to be related to the average half lengths of the micro-cracks on the interfaces by $\rho_{\mathrm{I}} / \rho_{\mathrm{II}}=\widehat{a}_{\mathrm{I}} / \widehat{a}_{\mathrm{II}}$, where $\widehat{a}_{\mathrm{I}}$ and $\widehat{a}_{\mathrm{II}}$ are the average half lengths of the micro-cracks on interfaces I and II respectively.

As in Sections 3.2 and 4.2, if the micro-cracked interfaces are replaced by spring-like interfaces with interfacial conditions (1.1) then we are interested in estimating the effective stiffness coefficients of the micro-cracked interfaces. 


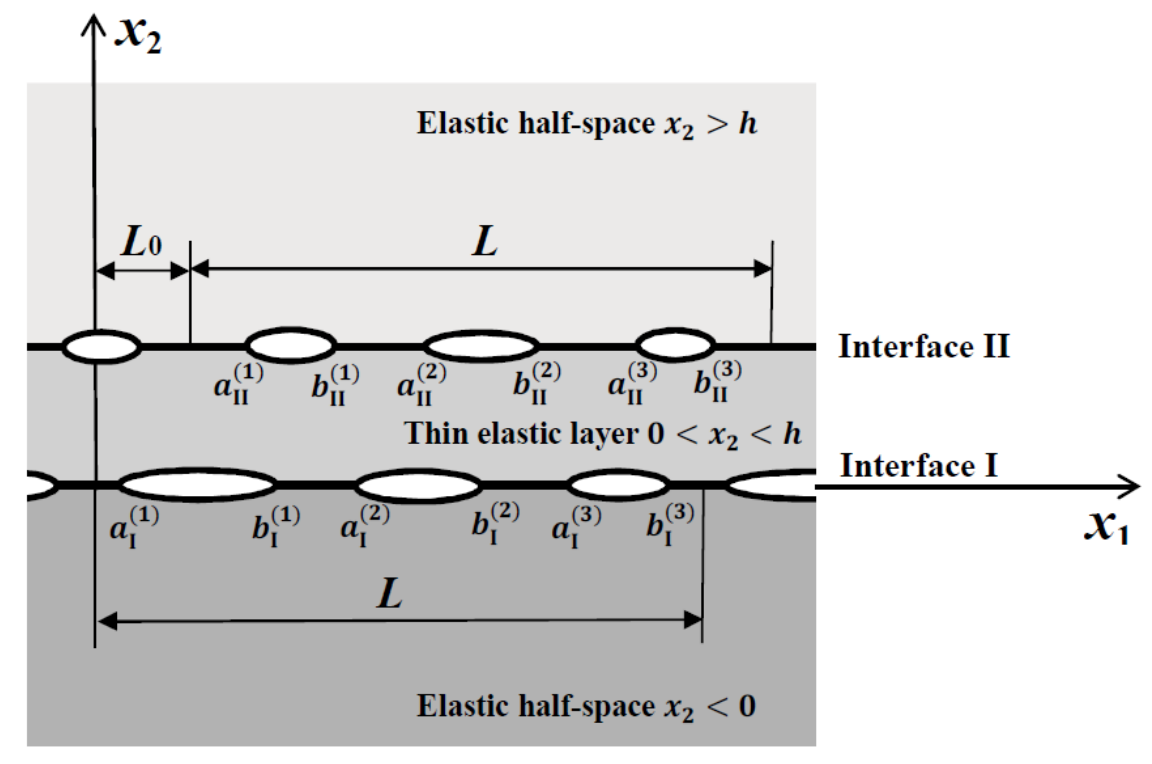

Figure 5.1: A sketch of the geometry of the trimaterial for $M=3$.

Specifically, the spring-like interfacial conditions for interfaces I and II are given by

$$
\begin{gathered}
{\left[\begin{array}{ll}
k_{11 \mathrm{I}} & k_{12 \mathrm{I}} \\
k_{21 \mathrm{I}} & k_{22 \mathrm{I}}
\end{array}\right]\left[\begin{array}{l}
\Delta u_{1 \mathrm{II}}\left(x_{1}\right) \\
\Delta u_{2 \mathrm{I}}\left(x_{1}\right)
\end{array}\right]=\left[\begin{array}{c}
\sigma_{12}\left(x_{1}, 0^{+}\right) \\
\sigma_{22}\left(x_{1}, 0^{+}\right)
\end{array}\right]=\left[\begin{array}{l}
\sigma_{12}\left(x_{1}, 0^{-}\right) \\
\sigma_{22}\left(x_{1}, 0^{-}\right)
\end{array}\right],} \\
{\left[\begin{array}{ll}
k_{11 \mathrm{II}} & k_{12 \mathrm{II}} \\
k_{21 \mathrm{II}} & k_{22 \mathrm{II}}
\end{array}\right]\left[\begin{array}{l}
\Delta u_{1 \mathrm{II}}\left(x_{1}\right) \\
\Delta u_{2 \mathrm{II}}\left(x_{1}\right)
\end{array}\right]=\left[\begin{array}{c}
\sigma_{12}\left(x_{1}, h^{+}\right) \\
\sigma_{22}\left(x_{1}, h^{+}\right)
\end{array}\right]=\left[\begin{array}{l}
\sigma_{12}\left(x_{1}, h^{-}\right) \\
\sigma_{22}\left(x_{1}, h^{-}\right)
\end{array}\right],} \\
\text {for inplane deformations, }
\end{gathered}
$$

and

$$
\begin{aligned}
& k_{33 \mathrm{I}} \Delta u_{3 \mathrm{I}}\left(x_{1}\right)= \sigma_{32}\left(x_{1}, 0^{+}\right)=\sigma_{32}\left(x_{1}, 0^{-}\right), \\
& k_{33 \mathrm{II}} \Delta u_{3 \mathrm{II}}\left(x_{1}\right)= \sigma_{32}\left(x_{1}, h^{+}\right)=\sigma_{32}\left(x_{1}, h^{-}\right), \\
& \text {for antiplane deformations, }
\end{aligned}
$$

where $\Delta u_{r \mathrm{I}}\left(x_{1}\right)=u_{r}\left(x_{1}, 0^{+}\right)-u_{r}\left(x_{1}, 0^{-}\right)$and $\Delta u_{3 \mathrm{I}}\left(x_{1}\right)=u_{3}\left(x_{1}, 0^{+}\right)-u_{3}\left(x_{1}, 0^{-}\right)$ are the displacement jumps across interface I, $\Delta u_{r \mathrm{II}}\left(x_{1}\right)=u_{r}\left(x_{1}, h^{+}\right)-u_{r}\left(x_{1}, h^{-}\right)$ 
and $\Delta u_{3 \mathrm{II}}\left(x_{1}\right)=u_{3}\left(x_{1}, h^{+}\right)-u_{3}\left(x_{1}, h^{-}\right)$are the displacement jumps across interface II, $u_{r}, \sigma_{r 2}, u_{3}$ and $\sigma_{32}$ are the displacements and stresses defined under (3.4), $k_{i j \mathrm{I}}$ and $k_{33 \mathrm{I}}$ denote the effective stiffness coefficients of interface I and $k_{i j \mathrm{II}}$ and $k_{33 \mathrm{II}}$ denote the effective stiffness coefficients of interface II.

The problem of interest here is to investigate the effects of the interaction of the pair of micro-cracked interfaces on the effective stiffness coefficients $k_{i j \mathrm{I}}$, $k_{33 \mathrm{I}}, k_{i j \mathrm{II}}$ and $k_{33 \mathrm{II}}$.

\subsection{Boundary Value Problems}

The trimaterial is subject to an external load at infinity. The external load is either antiplane or inplane. The micro-cracks along interfaces I and II are assumed to be traction-free. The displacements and the stresses are continuous across the uncracked parts of the interfaces.

As in (3.5) and (4.2), we write the antiplane displacement $u_{3}$ as the superposition of $u_{3}^{(\mathrm{ext})}$ and $u_{3}^{(\mathrm{imp})}$ and write the antiplane stress $\sigma_{32}$ as the superposition of $\sigma_{32}^{(\text {ext })}$ and $\sigma_{32}^{(\mathrm{imp})}$, where $u_{3}^{(\mathrm{ext})}$ and $\sigma_{32}^{(\mathrm{ext})}$ are respectively the displacement and the stress fields in the trimaterial for the corresponding case where interfaces I and II do not contain any micro-crack, and $u_{3}^{(\mathrm{imp})}$ and $\sigma_{32}^{(\mathrm{imp})}$ are elastic fields induced by the micro-cracks.

Similarly, the elastic fields of inplane displacements $u_{r}$ and stresses $\sigma_{r 2}$ are written as the superposition of the fields $u_{r}^{(\mathrm{ext})}, \sigma_{r 2}^{(\mathrm{ext})}$ for the case where the interfaces are wholly perfect and the fields $u_{r}^{(\mathrm{imp})}, \sigma_{r 2}^{(\mathrm{imp})}$ induced by the micro-cracks as given in (3.21) and (4.3).

The load acting on the trimaterial at infinity is assumed to be such that all the elastic fields $u_{3}^{(\mathrm{ext})}, \sigma_{32}^{(\mathrm{ext})}, u_{3}^{(\mathrm{imp})}$ and $\sigma_{32}^{(\mathrm{imp})}$ (for antiplane deformations) and $u_{r}^{(\mathrm{ext})}, \sigma_{r 2}^{(\mathrm{ext})}, u_{r}^{(\mathrm{imp})}$ and $\sigma_{r 2}^{(\mathrm{imp})}$ (for inplane deformations) are periodic functions 


\section{Micromechanical Models for Weak Interfaces}

of $x_{1}$ with period $L$ on any plane $x_{2}=c$ (constant).

For antiplane and inplane deformations, the boundary value problems to solve for estimating the effective stiffness coefficients of interfaces I and II are stated below.

\subsubsection{Antiplane Deformations}

For antiplane deformations, the conditions for the micro-cracked interfaces I and II are given by

$$
\begin{aligned}
& \sigma_{32}^{(\mathrm{imp})}\left(x_{1}, 0^{ \pm}\right)=-\sigma_{32}^{(\mathrm{ext})}\left(x_{1}, 0^{ \pm}\right) \text {for }\left(x_{1}, 0\right) \in D_{\mathrm{I}} \text {, } \\
& \sigma_{32}^{(\mathrm{imp})}\left(x_{1}, h^{ \pm}\right)=-\sigma_{32}^{(\mathrm{ext})}\left(x_{1}, h^{ \pm}\right) \text {for }\left(x_{1}, h\right) \in D_{\mathrm{II}}, \\
& \left.\begin{array}{rl}
\Delta u_{3 \mathrm{I}}^{(\mathrm{imp})}\left(x_{1}\right) & =0 \\
\sigma_{32}^{(\mathrm{imp})}\left(x_{1}, 0^{+}\right) & =\sigma_{32}^{(\mathrm{imp})}\left(x_{1}, 0^{-}\right)
\end{array}\right\} \text {for }\left(x_{1}, 0\right) \in P_{\mathrm{I}}, \\
& \left.\begin{array}{rl}
\Delta u_{3 \mathrm{II}}^{(\mathrm{imp})}\left(x_{1}\right) & =0 \\
\sigma_{32}^{(\mathrm{imp})}\left(x_{1}, h^{+}\right) & =\sigma_{32}^{(\mathrm{imp})}\left(x_{1}, h^{-}\right)
\end{array}\right\} \text {for }\left(x_{1}, h\right) \in P_{\mathrm{II}},
\end{aligned}
$$

where $\Delta u_{3 \mathrm{I}}^{(\mathrm{imp})}\left(x_{1}\right)$ and $\Delta u_{3 \mathrm{II}}^{(\mathrm{imp})}\left(x_{1}\right)$ are respectively defined by $\Delta u_{3 \mathrm{I}}^{(\mathrm{imp})}\left(x_{1}\right)=$ $u_{3}^{(\mathrm{imp})}\left(x_{1}, 0^{+}\right)-u_{3}^{(\mathrm{imp})}\left(x_{1}, 0^{-}\right)$and $\Delta u_{3 \mathrm{II}}^{(\mathrm{imp})}\left(x_{1}\right)=u_{3}^{(\mathrm{imp})}\left(x_{1}, h^{+}\right)-u_{3}^{(\mathrm{imp})}\left(x_{1}, h^{-}\right)$, $D_{\text {I }}$ and $D_{\text {II }}$ denote the micro-cracked parts of interfaces I and II respectively and $P_{\mathrm{I}}$ and $P_{\mathrm{II}}$ denote the perfectly bonded parts of interfaces I and II respectively.

With $u_{3}^{(\mathrm{ext})}$ and $\sigma_{32}^{(\mathrm{ext})}$ assumed given, the boundary value problem of interest here is to solve the partial differential equation (2.5) together with (5.1) subject to the interfacial conditions in (5.5) and the far field condition given by $\sigma_{32}^{(\mathrm{imp})} \rightarrow 0$ as $\left|x_{2}\right| \rightarrow \infty$.

Once the displacement jumps $\Delta u_{3 \mathrm{I}}^{(\mathrm{imp})}\left(x_{1}\right)$ and $\Delta u_{3 \mathrm{II}}^{(\mathrm{imp})}\left(x_{1}\right)$ across the microcracks are determined, the effective stiffness $k_{33 \text { I }}$ and $k_{33 I}$ may be estimated 
by using

$$
\begin{aligned}
k_{33 \mathrm{I}} \sum_{k=1}^{M} \int_{a_{\mathrm{I}}^{(k)}}^{b_{\mathrm{I}}^{(k)}} \Delta u_{3 \mathrm{I}}^{(\mathrm{imp})}\left(x_{1}\right) d x_{1} & =\int_{0}^{L} \sigma_{32}^{(\mathrm{ext})}\left(x_{1}, 0\right) d x_{1}, \\
k_{33 \mathrm{II}} \sum_{k=1}^{M} \int_{a_{\mathrm{II}}^{(k)}}^{b_{\mathrm{II}}^{(k)}} \Delta u_{3 \mathrm{II}}^{(\mathrm{imp})}\left(x_{1}\right) d x_{1} & =\int_{L_{0}}^{L_{0}+L} \sigma_{32}^{(\mathrm{ext})}\left(x_{1}, h\right) d x_{1} .
\end{aligned}
$$

\subsubsection{Inplane Deformations}

For inplane deformations, the interfacial conditions are given by

$$
\begin{aligned}
& \sigma_{r 2}^{(\mathrm{imp})}\left(x_{1}, 0^{ \pm}\right)=-\sigma_{r 2}^{(\mathrm{ext})}\left(x_{1}, 0\right) \text { for }\left(x_{1}, 0\right) \in D_{\mathrm{I}}, \\
& \sigma_{r 2}^{(\mathrm{imp})}\left(x_{1}, h^{ \pm}\right)=-\sigma_{r 2}^{(\mathrm{ext})}\left(x_{1}, h\right) \text { for }\left(x_{1}, h\right) \in D_{\mathrm{II}}, \\
& \left.\begin{array}{rl}
\Delta u_{r \mathrm{I}}^{(\mathrm{imp})}\left(x_{1}\right) & =0 \\
\sigma_{r 2}^{(\mathrm{imp})}\left(x_{1}, 0^{+}\right) & =\sigma_{r 2}^{(\mathrm{imp})}\left(x_{1}, 0^{-}\right)
\end{array}\right\} \text {for }\left(x_{1}, 0\right) \in P_{\mathrm{I}}, \\
& \left.\begin{array}{rl}
\Delta u_{r \mathrm{II}}^{(\mathrm{imp})}\left(x_{1}\right) & =0 \\
\sigma_{r 2}^{(\mathrm{imp})}\left(x_{1}, h^{+}\right) & =\sigma_{r 2}^{(\mathrm{imp})}\left(x_{1}, h^{-}\right)
\end{array}\right\} \text {for }\left(x_{1}, h\right) \in P_{\mathrm{II}},
\end{aligned}
$$

where $\Delta u_{r \mathrm{I}}^{(\mathrm{imp})}\left(x_{1}\right)$ and $\Delta u_{r \mathrm{II}}^{(\mathrm{imp})}\left(x_{1}\right)$ are respectively given by $\Delta u_{r \mathrm{I}}^{(\mathrm{imp})}\left(x_{1}\right)=$ $u_{r}^{(\mathrm{imp})}\left(x_{1}, 0^{+}\right)-u_{r}^{(\mathrm{imp})}\left(x_{1}, 0^{-}\right)$and $\Delta u_{r \mathrm{II}}^{(\mathrm{imp})}\left(x_{1}\right)=u_{r}^{(\mathrm{imp})}\left(x_{1}, h^{+}\right)-u_{r}^{(\mathrm{imp})}\left(x_{1}, h^{-}\right)$.

Thus, the boundary value problem is to solve the equilibrium equations in (2.10) together with (5.1) for $u_{k}^{(\mathrm{imp})}$ subject to the interfacial conditions in (5.7). In addition to (5.5), it is required that $\sigma_{k j}^{(\mathrm{imp})} \rightarrow 0$ as $\left|x_{2}\right| \rightarrow \infty$.

Once the boundary value problem is solved, the effective stiffness coefficients $k_{r j \mathrm{I}}$ and $k_{r j \mathrm{II}}$ of the micro-cracked interfaces by averaging the displacement jumps and the external stresses on the interfaces, that is, via the formulae:

$$
\begin{aligned}
& k_{r j \mathrm{I}} \sum_{k=1}^{M} \int_{a_{\mathrm{I}}^{(k)}}^{b_{\mathrm{I}}^{(k)}} \Delta u_{j \mathrm{I}}^{(\mathrm{imp})}\left(x_{1}\right) d x_{1}=\int_{0}^{L} \sigma_{r 2}^{(\mathrm{ext})}\left(x_{1}, 0\right) d x_{1}, \\
& k_{r j \mathrm{II}} \sum_{k=1}^{M} \int_{a_{\mathrm{II}}^{(k)}}^{b_{\mathrm{II}}^{(k)}} \Delta u_{j \mathrm{II}}^{(\mathrm{imp})}\left(x_{1}\right) d x_{1}=\int_{L_{0}}^{L_{0}+L} \sigma_{r 2}^{(\mathrm{ext})}\left(x_{1}, h\right) d x_{1} .
\end{aligned}
$$


In Section 5.4 below, the boundary value problems here for antiplane and inplane deformations are formulated in terms of hypersingular boundary integral equations with the displacement jumps $\Delta u_{3 \mathrm{I}}^{(\mathrm{imp})}\left(x_{1}\right), \Delta u_{3 \mathrm{II}}^{(\mathrm{imp})}\left(x_{1}\right)$, $\Delta u_{r \mathrm{I}}^{(\mathrm{imp})}\left(x_{1}\right)$ and $\Delta u_{r \mathrm{II}}^{(\mathrm{imp})}\left(x_{1}\right)$ across the micro-cracks as unknown functions to be determined. For estimating the effective stiffness coefficients, the loads in (5.6) and (5.8) are taken to be constants as given by

$$
\begin{aligned}
& \sigma_{32}^{\text {(ext) }}\left(x_{1}, 0^{ \pm}\right)=\sigma_{32}^{(\text {ext })}\left(x_{1}, h^{ \pm}\right)=\sigma_{3} \text { for antiplane deformations } \\
& \sigma_{r 2}^{\text {(ext) }}\left(x_{1}, 0^{ \pm}\right)=\sigma_{r 2}^{\text {ext) }}\left(x_{1}, h^{ \pm}\right)=\sigma_{r} \text { for inplane deformations. }
\end{aligned}
$$

For inplane deformations, two independent sets of loads are required to determine effective stiffness coefficients as explained in Subsection 3.4.2.

\subsection{Hypersingular Boundary Integro-differential Equations}

To formulate the boundary value problems in Section 5.3 above in terms of hypersingular boundary integro-differential equations, the trimaterial in Figure 5.1 is divided up into two separate subdomains defined by $x_{2}>h / 2$ and $x_{2}<h / 2$. The boundary integral equations given in Section 2.3 are then used together with the corresponding perfect interface Green's functions in Section 2.4 to derive hypersingular boundary integro-differential equations separately for each of the subdomains. The analysis in Subsection 4.3.1 is closely followed here to derive the hypersingular boundary integro-differential equations.

\subsubsection{Antiplane Deformations}

From the boundary integral equations for the lower subdomain $x_{2}<h / 2$, the hypersingular boundary integral equations for the stress $\sigma_{32}^{(\mathrm{imp})}$ on the artificial 
boundary $x_{2}=h^{-} / 2$ and interface I (where $x_{2}=0^{+}$) are respectively given by

$$
\begin{aligned}
& \frac{1}{2} \sigma_{32}^{(\mathrm{imp})}\left(\xi_{1}, h^{-} / 2\right) \\
= & -\frac{1}{2 \pi} f_{0}^{L} u_{3}^{(\mathrm{imp})}\left(x_{1}, h^{-} / 2\right)\left[\frac{1}{\left(x_{1}-\xi_{1}\right)^{2}}+\Theta\left(x_{1}, \xi_{1}\right)\right] d x_{1} \\
& +\frac{1}{2 \pi} \frac{\beta^{(2)}-\beta^{(3)}}{\beta^{(2)}+\beta^{(3)}} \int_{0}^{L} u_{3}^{(\mathrm{imp})}\left(x_{1}, h^{-} / 2\right) \operatorname{Re}\left\{\Omega\left(x_{1}, \xi_{1},\left(\tau^{(2)}-\bar{\tau}^{(2)}\right) h^{-} / 2\right)\right\} d x_{1} \\
& -\frac{\beta^{(2)}-\beta^{(3)}}{2 \pi\left(\beta^{(2)}+\beta^{(3)}\right)} \int_{0}^{L} \sigma_{32}^{(\mathrm{imp})}\left(x_{1}, h^{-} / 2\right) \operatorname{Re}\left\{\Gamma_{0}\left(x_{1}, \xi_{1},\left(\tau^{(2)}-\bar{\tau}^{(2)}\right) h^{-} / 2\right)\right\} d x_{1} \\
& +\frac{\beta^{(3)}}{\pi\left(\beta^{(2)}+\beta^{(3)}\right)} \sum_{k=1}^{M} \int_{a_{1}^{(k)}}^{b_{1}^{(k)}} \Delta u_{3 \mathrm{I}}^{(\mathrm{imp})}\left(x_{1}\right) \operatorname{Re}\left\{\Omega\left(x_{1}, \xi_{1},-\tau^{(2)} h^{-} / 2\right)\right\} d x_{1} \\
& \text { for } 0<\xi_{1}<L,
\end{aligned}
$$

and

$$
\begin{aligned}
& \int_{0}^{L} u_{3}^{(\mathrm{imp})}\left(x_{1}, h^{-} / 2\right) \operatorname{Re}\left\{\Omega\left(x_{1}, \xi_{1}, \tau^{(2)} h^{-} / 2\right)\right\} d x_{1} \\
& -\int_{0}^{L} \sigma_{32}^{(\mathrm{imp})}\left(x_{1}, h^{-} / 2\right) \operatorname{Re}\left\{\Gamma_{0}\left(x_{1}, \xi_{1}, \tau^{(2)} h^{-} / 2\right)\right\} d x_{1} \\
& -\sum_{k=1}^{M} f_{a_{\mathrm{I}}^{(k)}}^{b_{\mathrm{I}}^{(k)}} \Delta u_{3 \mathrm{I}}^{(\mathrm{imp})}\left(x_{1}\right)\left[\frac{1}{\left(x_{1}-\xi_{1}\right)^{2}}+\Theta\left(x_{1}, \xi_{1}\right)\right] d x_{1} \\
= & \frac{\pi}{\beta^{(3)}}\left(\beta^{(2)}+\beta^{(3)}\right) \sigma_{3} \\
& \text { for } a_{\mathrm{I}}^{(n)}<\xi_{1}<b_{\mathrm{I}}^{(n)}(n=1,2, \ldots, M),
\end{aligned}
$$

where $\beta^{(p)}$ and $\tau^{(p)}$ are defined below (2.32), $\Theta\left(x_{1}, \xi_{1}\right)$ is defined in (3.20), $\Omega\left(x_{1}, \xi_{1}, z\right)$ is given in (4.15), $i=\sqrt{-1}$, and $\Gamma_{0}\left(x_{1}, \xi_{1}, z\right)$ is defined by

$$
\begin{aligned}
\Gamma_{0}\left(x_{1}, \xi_{1}, z\right)= & \frac{i}{x_{1}-\xi_{1}+z}-\frac{i}{L} \Psi_{0}\left(\frac{L+x_{1}-\xi_{1}+z}{L}\right) \\
& +\frac{i}{L} \Psi_{0}\left(\frac{L-x_{1}+\xi_{1}-z}{L}\right),
\end{aligned}
$$

with $\Psi_{0}(z)$ being the digamma function as defined in (2.45). Note that (5.11) is derived from the interfacial conditions on $D_{\mathrm{I}}$ and $P_{\mathrm{I}}$ in (5.5). 
From the boundary integral equations for the upper subdomain $x_{2}>h / 2$, the hypersingular integral equations for the stress $\sigma_{32}^{(\mathrm{imp})}$ on the artificial boundary $x_{2}=h^{+} / 2$ and interface II (where $x_{2}=h^{-}$) are given by

$$
\begin{aligned}
& \frac{1}{2} \sigma_{32}^{(\mathrm{imp})}\left(\xi_{1}, h^{+} / 2\right) \\
= & \frac{1}{2 \pi} f_{L_{0}}^{L_{0}+L} u_{3}^{(\mathrm{imp})}\left(x_{1}, h^{+} / 2\right)\left[\frac{1}{\left(x_{1}-\xi_{1}\right)^{2}}+\Theta\left(x_{1}, \xi_{1}\right)\right] d x_{1} \\
& -\frac{\beta^{(2)}-\beta^{(1)}}{2 \pi\left(\beta^{(2)}+\beta^{(1)}\right)} \int_{L_{0}}^{L_{0}+L} u_{3}^{(\mathrm{imp})}\left(x_{1}, h^{+} / 2\right) \\
& \times \operatorname{Re}\left\{\Omega\left(x_{1}, \xi_{1},-\left(\tau^{(2)}-\bar{\tau}^{(2)}\right) h^{+} / 2\right)\right\} d x_{1} \\
& +\frac{\beta^{(2)}-\beta^{(1)}}{2 \pi\left(\beta^{(2)}+\beta^{(1)}\right)} \int_{L_{0}}^{L_{0}+L} \sigma_{32}^{(\mathrm{imp})}\left(x_{1}, h^{+} / 2\right) \\
& \times \operatorname{Re}\left\{\Gamma_{0}\left(x_{1}, \xi_{1},-\left(\tau^{(2)}-\bar{\tau}^{(2)}\right) h^{+} / 2\right)\right\} d x_{1} \\
& +\frac{\beta^{(1)}}{\pi\left(\beta^{(2)}+\beta^{(1)}\right)} \sum_{k=1}^{M} \int_{a_{\mathrm{II}}^{(k)}}^{b_{\mathrm{II}}^{(k)}} \Delta u_{3 \mathrm{II}}^{(\mathrm{imp})}\left(x_{1}\right) \operatorname{Re}\left\{\Omega\left(x_{1}, \xi_{1}, \tau^{(2)} h^{+} / 2\right)\right\} d x_{1} \\
& \text { for } L_{0}<\xi_{1}<L_{0}+L,
\end{aligned}
$$

and

$$
\begin{aligned}
& \int_{L_{0}}^{L_{0}+L} u_{3}^{(\mathrm{imp})}\left(x_{1}, h^{+} / 2\right) \operatorname{Re}\left\{\Omega\left(x_{1}, \xi_{1},-\tau^{(2)} h^{+} / 2\right)\right\} d x_{1} \\
& -\int_{L_{0}}^{L_{0}+L} \sigma_{32}^{(\mathrm{imp})}\left(x_{1}, h^{+} / 2\right) \operatorname{Re}\left\{\Gamma_{0}\left(x_{1}, \xi_{1},-\tau^{(2)} h^{+} / 2\right)\right\} d x_{1} \\
& +\sum_{k=1}^{M} f_{a_{\mathrm{II}}^{(k)}}^{b_{\mathrm{II}}^{(k)}} \Delta u_{3 \mathrm{II}}^{(\mathrm{imp})}\left(x_{1}\right)\left[\frac{1}{\left(x_{1}-\xi_{1}\right)^{2}}+\Theta\left(x_{1}, \xi_{1}\right)\right] d x_{1} \\
= & -\frac{\pi}{\beta^{(1)}}\left(\beta^{(2)}+\beta^{(1)}\right) \sigma_{3} \\
& \text { for } a_{\mathrm{II}}^{(n)}<\xi_{1}<b_{\mathrm{II}}^{(n)}(n=1,2, \ldots, M) .
\end{aligned}
$$

Note that (5.10), (5.11), (5.13) and (5.14) do not contain any integral over the perfect (uncracked) parts of interfaces I and II because of the use of the special perfect interface Green's function. 
In (5.10), (5.11), (5.13) and (5.14), the unknown functions are the displacement jumps $\Delta u_{3 \mathrm{I}}^{(\mathrm{imp})}\left(x_{1}\right)$ and $\Delta u_{3 \mathrm{II}}^{(\mathrm{imp})}\left(x_{1}\right)$ across the micro-cracks on interfaces I and II respectively, the displacements $u_{3}^{\text {(imp) }}\left(x_{1}, h^{+} / 2\right)$ and $u_{3}^{(\mathrm{imp})}\left(x_{1}, h^{-} / 2\right)$ and the stresses $\sigma_{32}^{(\mathrm{imp})}\left(x_{1}, h^{+} / 2\right)$ and $\sigma_{32}^{(\mathrm{imp})}\left(x_{1}, h^{-} / 2\right)$. There are six unknown functions but only four equations. To complete the formulation, continuity conditions are imposed on the artificially created boundary $x_{2}=h / 2$ as follows:

$$
\left.\begin{array}{l}
u_{3}^{(\mathrm{imp})}\left(x_{1}, h^{+} / 2\right)=u_{3}^{(\mathrm{imp})}\left(x_{1}, h^{-} / 2\right) \\
\sigma_{32}^{(\mathrm{imp})}\left(x_{1}, h^{+} / 2\right)=\sigma_{32}^{(\mathrm{mp})}\left(x_{1}, h^{-} / 2\right)
\end{array}\right\} \text { for }-\infty<x_{1}<\infty .
$$

Note that $\Delta u_{3 \mathrm{I}}^{(\mathrm{imp})}\left(x_{1}\right), \Delta u_{3 \mathrm{II}}^{(\mathrm{imp})}\left(x_{1}\right), u_{3}^{(\mathrm{imp})}\left(x_{1}, h^{ \pm} / 2\right)$ and $\sigma_{32}^{(\mathrm{imp})}\left(x_{1}, h^{ \pm} / 2\right)$ are all periodic functions of $x_{1}$ with period $L$.

As $h \rightarrow \infty$, the equations (5.10) and (5.13) disappear and both (5.11) and (5.14) reduce to (3.19) which corresponds to the case where the micro-cracked interface lies between two dissimilar half-spaces.

Once $\Delta u_{3 \mathrm{I}}^{(\mathrm{imp})}\left(x_{1}\right)$ and $\Delta u_{3 \mathrm{II}}^{(\mathrm{imp})}\left(x_{1}\right)$ are obtained by solving the hypersingular boundary integral equations (5.10), (5.11), (5.13) and (5.14) together with (5.15), the effective stiffness coefficients $k_{33 \text { I }}$ and $k_{33 I I}$ for interfaces I and II may be estimated by using (5.6).

\subsubsection{Inplane Deformations}

From the analysis on the lower subdomain $x_{2}<h / 2$, the stress $\sigma_{i 2}^{(\text {imp) }}$ on the artificial boundary $x_{2}=h^{-} / 2$ is given by the hypersingular boundary integral 
equations

$$
\begin{aligned}
& \pi \sigma_{i 2}^{(\mathrm{imp})}\left(\xi_{1}, h^{-} / 2\right) \\
= & f_{0}^{L} u_{r}^{(\mathrm{imp})}\left(x_{1}, h^{-} / 2\right) \operatorname{Re}\left\{\sum_{\alpha=1}^{2} G_{r i \alpha}\right\}\left[\frac{1}{\left(x_{1}-\xi_{1}\right)^{2}}+\Theta\left(x_{1}, \xi_{1}\right)\right] d x_{1} \\
& +\int_{0}^{L} u_{r}^{(\mathrm{imp})}\left(x_{1}, h^{-} / 2\right) \operatorname{Re}\left\{\sum_{\alpha=1}^{2} \sum_{\beta=1}^{2} H_{\text {ria } \beta \mathrm{I}} \Omega\left(x_{1}, \xi_{1},\left(\tau_{\alpha}^{(2)}-\bar{\tau}_{\beta}^{(2)}\right) h^{-} / 2\right)\right\} d x_{1} \\
& +f_{0}^{L} \sigma_{r 2}^{(\mathrm{imp})}\left(x_{1}, h^{-} / 2\right) \operatorname{Re}\left\{\sum_{\alpha=1}^{2} E_{r i \alpha}\right\}\left[\frac{1}{x_{1}-\xi_{1}}+\Lambda\left(x_{1}, \xi_{1}\right)\right] d x_{1} \\
& +\int_{0}^{L} \sigma_{r 2}^{(\mathrm{imp})}\left(x_{1}, h^{-} / 2\right) \operatorname{Re}\left\{\sum_{\alpha=1}^{2} \sum_{\beta=1}^{2} F_{r i \alpha \beta \mathrm{I}} \Pi\left(x_{1}, \xi_{1},\left(\tau_{\alpha}^{(2)}-\bar{\tau}_{\beta}^{(2)}\right) h^{-} / 2\right)\right\} d x_{1} \\
& -\sum_{k=1}^{M} \int_{a_{\mathrm{I}}^{(k)}}^{b_{\mathrm{I}}^{(k)}} \Delta u_{r \mathrm{I}}^{(\mathrm{imp})}\left(x_{1}\right) \operatorname{Re}\left\{\sum_{\alpha=1}^{2} G_{r i \alpha} \Omega\left(x_{1}, \xi_{1},-\tau_{\alpha}^{(2)} h^{-} / 2\right)\right\} d x_{1} \\
& -\sum_{k=1}^{M} \int_{a_{\mathrm{I}}^{(k)}}^{b_{\mathrm{I}}^{(k)}} \Delta u_{r \mathrm{I}}^{(\mathrm{imp})}\left(x_{1}\right) \operatorname{Re}\left\{\sum_{\alpha=1}^{2} \sum_{\beta=1}^{2} H_{r i \alpha \beta \mathrm{I}} \Omega\left(x_{1}, \xi_{1},-\bar{\tau}_{\beta}^{(2)} h^{-} / 2\right)\right\} d x_{1} \\
& \text { for } 0<\xi_{1}<L,
\end{aligned}
$$

and the conditions on interface I, as given in (5.5), may be expressed in terms of the hypersingular boundary integro-differential equations

$$
\begin{aligned}
& \int_{0}^{L} u_{r}^{(\mathrm{imp})}\left(x_{1}, h^{-} / 2\right) \operatorname{Re}\left\{\sum_{\alpha=1}^{2} \Omega\left(x_{1}, \xi_{1}, \tau_{\alpha}^{(2)} h^{-} / 2\right)\left(G_{r i \alpha}+\sum_{\beta=1}^{2} H_{r i \alpha \beta \mathrm{I}}\right)\right\} d x_{1} \\
& +\int_{0}^{L} \sigma_{r 2}^{(\mathrm{imp})}\left(x_{1}, h^{-} / 2\right) \operatorname{Re}\left\{\sum_{\alpha=1}^{2} \Pi\left(x_{1}, \xi_{1}, \tau_{\alpha}^{(2)} h^{-} / 2\right)\left(E_{r i \alpha}+\sum_{\beta=1}^{2} F_{r i \alpha \beta \mathrm{I}}\right)\right\} d x_{1} \\
& -\sum_{k=1}^{M} f_{a_{\mathrm{I}}^{(k)}}^{b_{\mathrm{I}}^{(k)}} \Delta u_{r \mathrm{I}}\left(x_{1}\right) \operatorname{Re}\left\{W_{r i \mathrm{I}}\right\}\left[\frac{1}{\left(x_{1}-\xi_{1}\right)^{2}}+\Theta\left(x_{1}, \xi_{1}\right)\right] d x_{1} \\
& +\operatorname{Im}\left\{\pi V_{r i \mathrm{I}}\right\} \frac{d}{d \xi_{1}}\left[\Delta u_{r \mathrm{I}}\left(\xi_{1}\right)\right] \\
& =-2 \pi \sigma_{i} \text { for } a_{\mathrm{I}}^{(n)}<\xi_{1}<b_{\mathrm{I}}^{(n)}(n=1,2, \ldots, M),
\end{aligned}
$$


where the constants $G_{r i \alpha}, H_{r i \alpha \beta \mathrm{I}}, W_{r i \mathrm{I}}, V_{r i \mathrm{I}}, E_{r i \alpha}$ and $F_{r i \alpha \beta \mathrm{I}}$ are defined by

$$
\begin{aligned}
G_{r i \alpha} & =\left[L_{r 2 \alpha}^{(2)} N_{\alpha p}^{(2)}\left(c_{i 2 m 1}^{(2)}+\tau_{\alpha}^{(2)} c_{i 2 m 2}^{(2)}\right)\right] D_{p m}^{(2)}, \\
H_{r i \alpha \beta \mathrm{I}} & =\left[L_{r 2 \alpha}^{(2)} Q_{\alpha \beta p}^{(2)}\left(c_{i 2 m 1}^{(2)}+\bar{\tau}_{\beta}^{(2)} c_{i 2 m 2}^{(2)}\right)\right] D_{p m}^{(2)}, \\
W_{r i \mathrm{I}} & =\sum_{\alpha=1}^{2} G_{r i \alpha}+\sum_{\alpha=1}^{2} \sum_{\beta=1}^{2} H_{r i \alpha \beta \mathrm{I}}, \\
V_{r i \mathrm{I}} & =\sum_{\alpha=1}^{2} \sum_{\beta=1}^{2} H_{r i \alpha \beta \mathrm{I}}-\sum_{\alpha=1}^{2} G_{r i \alpha}, \\
E_{r i \alpha} & =\left[A_{r \alpha}^{(2)} N_{\alpha p}^{(2)}\left(c_{i 2 m 1}^{(2)}+\tau_{\alpha}^{(2)} c_{i 2 m 2}^{(2)}\right)\right] D_{p m}^{(2)}, \\
F_{r i \alpha \beta \mathrm{I}} & =\left[A_{r \alpha}^{(2)} Q_{\alpha \beta p}^{(2)}\left(c_{i 2 m 1}^{(2)}+\bar{\tau}_{\beta}^{(2)} c_{i 2 m 2}^{(2)}\right)\right] D_{p m}^{(2)},
\end{aligned}
$$

the constants $c_{i j k l}^{(q)}, \tau_{\alpha}^{(q)}, A_{m \alpha}^{(q)}, L_{i 2 \alpha}^{(q)}, N_{m \alpha}^{(q)}$ and $D_{p m}^{(q)}$ are defined below (2.39), the constants $Q_{\alpha \beta p}^{(2)}$ are implicitly defined by

$$
\sum_{\alpha=1}^{2}\left[N_{\gamma r}^{(3)} \bar{A}_{r \alpha}^{(2)}-M_{\gamma r}^{(3)} \bar{L}_{r 2 \alpha}^{(2)}\right] \bar{Q}_{\alpha \beta p}^{(2)}=\left[M_{\gamma r}^{(3)} L_{r 2 \beta}^{(2)}-N_{\gamma r}^{(3)} A_{r \beta}^{(2)}\right] N_{\beta p}^{(2)}
$$

with $M_{\gamma r}^{(q)}$ defined below (2.39) and the functions $\Lambda\left(x_{1}, \xi_{1}\right)$ and $\Pi\left(x_{1}, \xi_{1}, z\right)$ are defined by

$$
\begin{aligned}
\Lambda\left(x_{1}, \xi_{1}\right)= & \frac{1}{L} \Psi_{0}\left(\frac{L-x_{1}+\xi_{1}}{L}\right)-\frac{1}{L} \Psi_{0}\left(\frac{L+x_{1}-\xi_{1}}{L}\right), \\
\Pi\left(x_{1}, \xi_{1}, z\right)= & \frac{1}{x_{1}-\xi_{1}+z}+\frac{1}{L} \Psi_{0}\left(\frac{L-x_{1}+\xi_{1}-z}{L}\right) \\
& -\frac{1}{L} \Psi_{0}\left(\frac{L+x_{1}-\xi_{1}+z}{L}\right) .
\end{aligned}
$$

Note that (5.18) is obtained by replacing superscripts 1 and 2 in (2.38) respectively by 2 and 3 .

Similarly, from the analysis on the upper subdomain $x_{2}>h / 2$, the stress $\sigma_{i 2}^{(\mathrm{imp})}$ on the artificial boundary $x_{2}=h^{+} / 2$ is given by the hypersingular 
boundary integral equations

$$
\begin{aligned}
& \pi \sigma_{i 2}^{(\mathrm{imp})}\left(\xi_{1}, h^{+} / 2\right) \\
= & -f_{L_{0}}^{L_{0}+L} u_{r}^{(\mathrm{imp})}\left(x_{1}, h^{+} / 2\right) \operatorname{Re}\left\{\sum_{\alpha=1}^{2} G_{r i \alpha}\right\}\left[\frac{1}{\left(x_{1}-\xi_{1}\right)^{2}}+\Theta\left(x_{1}, \xi_{1}\right)\right] d x_{1} \\
& -\int_{L_{0}}^{L_{0}+L} u_{r}^{(\mathrm{imp})}\left(x_{1}, h^{+} / 2\right) \operatorname{Re}\left\{\sum_{\alpha=1}^{2} \sum_{\beta=1}^{2} H_{r i \alpha \beta \mathrm{II}} \Omega\left(x_{1}, \xi_{1},-\left(\tau_{\alpha}^{(2)}-\bar{\tau}_{\beta}^{(2)}\right) h / 2\right)\right\} d x_{1} \\
& -f_{L_{0}}^{L_{0}+L} \sigma_{r 2}^{(\mathrm{imp})}\left(x_{1}, h^{+} / 2\right) \operatorname{Re}\left\{\sum_{\alpha=1}^{2} E_{r i \alpha}\right\}\left[\frac{1}{x_{1}-\xi_{1}}+\Lambda\left(x_{1}, \xi_{1}\right)\right] d x_{1} \\
& -\int_{L_{0}}^{L_{0}+L} \sigma_{r 2}^{(\mathrm{imp})}\left(x_{1}, h^{+} / 2\right) \operatorname{Re}\left\{\sum_{\alpha=1}^{2} \sum_{\beta=1}^{2} F_{r i \alpha \beta \mathrm{II}} \Pi\left(x_{1}, \xi_{1},-\left(\tau_{\alpha}^{(2)}-\bar{\tau}_{\beta}^{(2)}\right) h / 2\right)\right\} d x_{1} \\
& -\sum_{k=1}^{M} \int_{a_{\mathrm{II}}^{(k)}}^{b_{\mathrm{II}}^{(k)}} \Delta u_{r \mathrm{II}}^{(\mathrm{imp})}\left(x_{1}\right) \operatorname{Re}\left\{\sum_{\alpha=1}^{2} G_{r i \alpha} \Omega\left(x_{1}, \xi_{1}, \tau_{\alpha}^{(2)} h / 2\right)\right\} d x_{1} \\
& -\sum_{k=1}^{M} \int_{a_{\mathrm{II}}^{(k)}}^{b_{\mathrm{II}}^{(k)}} \Delta u_{r \mathrm{II}}^{(\mathrm{imp})}\left(x_{1}\right) \operatorname{Re}\left\{\sum_{\alpha=1}^{2} \sum_{\beta=1}^{2} H_{r i \alpha \beta \mathrm{II}} \Omega\left(x_{1}, \xi_{1}, \bar{\tau}_{\beta}^{(2)} h / 2\right)\right\} d x_{1} \\
& \text { for } 0<\xi_{1}<L,
\end{aligned}
$$

and the conditions on interface II may be expressed in terms of the hypersingular boundary integro-differential equations

$$
\begin{aligned}
& \int_{L_{0}}^{L_{0}+L} u_{r}^{(\mathrm{imp})}\left(x_{1}, h^{+} / 2\right) \operatorname{Re}\left\{\sum_{\alpha=1}^{2} \Omega\left(x_{1}, \xi_{1},-\tau_{\alpha}^{(2)} h^{-} / 2\right)\left(G_{r i \alpha}+\sum_{\beta=1}^{2} H_{r i \alpha \beta \mathrm{II}}\right)\right\} d x_{1} \\
& +\int_{L_{0}}^{L_{0}+L} \sigma_{r 2}^{(\mathrm{imp})}\left(x_{1}, h^{+} / 2\right) \operatorname{Re}\left\{\sum_{\alpha=1}^{2} \Pi\left(x_{1}, \xi_{1},-\tau_{\alpha}^{(2)} h^{-} / 2\right)\left(E_{r i \alpha}+\sum_{\beta=1}^{2} F_{r i \alpha \beta \mathrm{II}}\right)\right\} d x_{1} \\
& +\sum_{k=1}^{M} f_{a_{\mathrm{II}}^{(k)}}^{b_{\mathrm{II}}^{(k)}} \Delta u_{r \mathrm{II}}\left(x_{1}\right) \operatorname{Re}\left\{W_{r i \mathrm{I}}\right\}\left[\frac{1}{\left(x_{1}-\xi_{1}\right)^{2}}+\Theta\left(x_{1}, \xi_{1}\right)\right] d x_{1} \\
& +\frac{d \Delta u_{r \mathrm{II}}\left(\xi_{1}\right)}{d \xi_{1}} \operatorname{Im}\left\{\pi V_{r i \mathrm{II}}\right\} \\
= & 2 \pi \sigma_{i} \text { for } a_{\mathrm{II}}^{(n)}<\xi_{1}<b_{\mathrm{II}}^{(n)}(n=1,2, \ldots, M),
\end{aligned}
$$


where $H_{r i \alpha \beta \mathrm{II}}, F_{r i \alpha \beta \mathrm{II}}, W_{r i \mathrm{II}}$ and $V_{r i \mathrm{II}}$ are given by

$$
\begin{aligned}
H_{r i \alpha \beta \mathrm{II}} & =\left[L_{r 2 \alpha}^{(2)} T_{\alpha \beta p}^{(2)}\left(c_{i 2 m 1}^{(2)}+\bar{\tau}_{\beta}^{(2)} c_{i 2 m 2}^{(2)}\right)\right] D_{p m}^{(2)}, \\
F_{r i \alpha \beta \mathrm{II}} & =\left[A_{r \alpha}^{(2)} T_{\alpha \beta p}^{(2)}\left(c_{i 2 m 1}^{(2)}+\bar{\tau}_{\beta}^{(2)} c_{i 2 m 2}^{(2)}\right)\right] D_{p m}^{(2)}, \\
W_{r i \mathrm{II}} & =\sum_{\alpha=1}^{2} G_{r i \alpha}+\sum_{\alpha=1}^{2} \sum_{\beta=1}^{2} H_{r i \alpha \beta \mathrm{II}}, \\
V_{r i \mathrm{II}} & =\sum_{\alpha=1}^{2} \sum_{\beta=1}^{2} H_{r i \alpha \beta \mathrm{II}}-\sum_{\alpha=1}^{2} G_{r i \alpha},
\end{aligned}
$$

and the constants $T_{\alpha \beta p}^{(2)}$ may be implicitly defined by

$$
\sum_{\alpha=1}^{2}\left[N_{\gamma k}^{(1)} \bar{A}_{k \alpha}^{(2)}-M_{\gamma k}^{(1)} \bar{L}_{k 2 \alpha}^{(2)}\right] \bar{T}_{\alpha \beta p}^{(2)}=\left[M_{\gamma k}^{(1)} L_{k 2 \beta}^{(2)}-N_{\gamma k}^{(1)} A_{k \beta}^{(2)}\right] N_{\beta p}^{(2)},
$$

which is obtained from (2.39).

As $h \rightarrow \infty,(5.16)$ and (5.19) disappear and both the equations (5.17) and (5.20) reduce to (3.30). Like in (4.17), if we compare (3.30) and the limiting case where $h \rightarrow \infty$ for (5.17) and (5.20), we may deduce that the constants $W_{r i \mathrm{I}}$ and $V_{r i \mathrm{I}}$ in (5.17) relate to the constants $Q_{i r}$ in (3.30) by

$$
\begin{aligned}
& \operatorname{Re}\left\{Q_{i r}\right\}=-\frac{\operatorname{Re}\left\{W_{i r \mathrm{I}}\right\}}{2 \pi}, \\
& \operatorname{Im}\left\{Q_{i r}\right\}=-\frac{\operatorname{Im}\left\{V_{i r \mathrm{I}}\right\}}{2 \pi} .
\end{aligned}
$$

Also, it can be deduced that the constants $W_{r i I I}$ and $V_{r i I I}$ in (5.20) are related to the constants $Q_{i r}$ in (3.30) by

$$
\begin{aligned}
& \operatorname{Re}\left\{Q_{i r}\right\}=-\frac{\operatorname{Re}\left\{W_{i r \mathrm{II}}\right\}}{2 \pi}, \\
& \operatorname{Im}\left\{Q_{i r}\right\}=-\frac{\operatorname{Im}\left\{V_{i r \mathrm{II}}\right\}}{2 \pi} .
\end{aligned}
$$

In (5.16), (5.17), (5.19) and (5.20), there are 12 unknown functions given by the displacements $u_{r}^{(\mathrm{imp})}\left(x_{1}, h^{+} / 2\right), u_{r}^{(\mathrm{imp})}\left(x_{1}, h^{-} / 2\right)$, the stresses $\sigma_{r 2}^{(\mathrm{imp})}\left(x_{1}, h^{+} / 2\right)$, 


\section{Micromechanical Models for Weak Interfaces}

$\sigma_{r 2}^{(\mathrm{imp})}\left(x_{1}, h^{-} / 2\right)$ and the displacement jumps $\Delta u_{r \mathrm{I}}^{(\mathrm{imp})}\left(x_{1}\right)$ and $\Delta u_{r \mathrm{II}}^{(\mathrm{imp})}\left(x_{1}\right)$ over interfaces I and II respectively. However, there are only 8 equations in (5.16), (5.17), (5.19) and (5.20). Four more equations are required to complete the formulation. They are given by the continuity conditions on the artificial boundary $x_{2}=h / 2$ as follows:

$$
\left.\begin{array}{l}
u_{r}^{(\mathrm{imp})}\left(x_{1}, h^{+} / 2\right)=u_{r}^{(\mathrm{imp})}\left(x_{1}, h^{-} / 2\right) \\
\sigma_{r 2}^{(\mathrm{imp})}\left(x_{1}, h^{+} / 2\right)=\sigma_{r 2}^{(\mathrm{imp})}\left(x_{1}, h^{-} / 2\right)
\end{array}\right\} \text { for }-\infty<x_{1}<\infty, r=1,2 .
$$

Once $\Delta u_{r \mathrm{I}}^{(\mathrm{imp})}\left(x_{1}\right)$ and $\Delta u_{r \mathrm{II}}^{(\mathrm{imp})}\left(x_{1}\right)$ are determined by solving (5.16), (5.17), (5.19) and (5.20) together with (5.15), the effective stiffness coefficients $k_{r j \mathrm{I}}$ and $k_{r j \mathrm{II}}$ for interfaces I and II respectively may be estimated by using (5.8).

\subsection{Numerical Procedures}

Numerical procedures for solving the hypersingular boundary integro-differential equations in Section 5.4 are outlined here.

For the numerical treatment of (5.10), (5.13), (5.16) and (5.19), the interval $0 \leq x_{1} \leq L_{0}$ is divided into $N_{0}$ equal length elements given by $x^{(p)} \leq x_{1} \leq$ $x^{(p+1)}$ for $p=1,2, \cdots, N_{0}$, the interval $L_{0} \leq x_{1} \leq L$ into $N_{1}$ equal length elements given by $x^{\left(N_{0}+p\right)} \leq x_{1} \leq x^{\left(N_{0}+p+1\right)}$ for $p=1,2, \cdots, N_{1}$, and the interval $L \leq x_{1} \leq L+L_{0}$ into $N_{0}$ equal length elements given by $x^{\left(N_{0}+N_{1}+p\right)} \leq$ $x_{1} \leq x^{\left(N_{0}+N_{1}+p+1\right)}$ for $p=1,2, \cdots, N_{0}$. Thus, the interval $0 \leq x_{1} \leq L+L_{0}$ is discretized into $2 N_{0}+N_{1}$ elements. For convenience, the element defined by $x^{(m)} \leq x_{1} \leq x^{(m+1)}$ is denoted by $E^{(m)}\left(m=1,2, \cdots, 2 N_{0}+N_{1}\right)$.

The unknown functions $u_{3}^{(\mathrm{imp})}\left(x_{1}, h^{-} / 2\right), \sigma_{32}^{(\mathrm{imp})}\left(x_{1}, h^{-} / 2\right), u_{r}^{(\mathrm{imp})}\left(x_{1}, h^{-} / 2\right)$ and $\sigma_{r 2}^{(\mathrm{imp})}\left(x_{1}, h^{-} / 2\right)$ are defined for $0 \leq x_{1} \leq L$, while the unknown functions $u_{3}^{(\mathrm{imp})}\left(x_{1}, h^{+} / 2\right), \sigma_{32}^{\text {(imp) }}\left(x_{1}, h^{+} / 2\right), u_{r}^{(\mathrm{imp})}\left(x_{1}, h^{+} / 2\right)$ and $\sigma_{r 2}^{(\mathrm{imp})}\left(x_{1}, h^{+} / 2\right)$ are defined for $L_{0} \leq x_{1} \leq L_{0}+L$. These unknown functions are approximated as 
explained below.

\subsubsection{Antiplane Deformations}

For antiplane deformations, the hypersingular boundary integral equations (5.10), (5.11), (5.13) and (5.14) are approximated as algebraic equations as explained below.

From (5.15), the functions $u_{3}^{(\mathrm{imp})}\left(x_{1}, h^{ \pm} / 2\right)$ and $\sigma_{32}^{(\mathrm{imp})}\left(x_{1}, h^{ \pm} / 2\right)$ may be approximated over the first $N_{0}+N_{1}$ elements (in the interval $0 \leq x_{1} \leq L$ ) by

$$
\left.\begin{array}{l}
u_{3}^{(\mathrm{imp})}\left(x_{1}, h^{ \pm} / 2\right) \simeq x_{1} \phi^{(m)}+\psi^{(m)} \\
\sigma_{32}^{(\mathrm{imp})}\left(x_{1}, h^{ \pm} / 2\right) \simeq x_{1} \vartheta^{(m)}+\eta^{(m)}
\end{array}\right\} \text { over } E^{(m)}\left(m=1,2, \cdots, N_{0}+N_{1}\right)
$$

where $\phi^{(m)}, \psi^{(m)}, \vartheta^{(m)}$ and $\eta^{(m)}$ are constants yet to be determined. Since $u_{3}^{(\mathrm{imp})}\left(x_{1}, h^{ \pm} / 2\right)$ and $\sigma_{32}^{(\mathrm{imp})}\left(x_{1}, h^{ \pm} / 2\right)$ are periodic functions of $x_{1}$ with period $L$, they may be approximated over the last $N_{0}$ elements (in $L \leq x_{1} \leq L+L_{0}$ ) by using

$$
\begin{aligned}
& \left.\begin{array}{l}
u_{3}^{(\mathrm{imp})}\left(x_{1}, h^{ \pm} / 2\right) \simeq\left(x_{1}-L\right) \phi^{(m)}+\psi^{(m)} \\
\sigma_{32}^{(\mathrm{imp})}\left(x_{1}, h^{ \pm} / 2\right) \simeq\left(x_{1}-L\right) \vartheta^{(m)}+\eta^{(m)}
\end{array}\right\} \text { over } E^{(m)} \\
& \left(m=N_{0}+N_{1}+1, N_{0}+N_{1}+2, \cdots, N_{0}+N_{1}+N_{0}\right) \text {. }
\end{aligned}
$$

The number of unknown constants in (5.25) and (5.26) is $4\left(N_{0}+N_{1}\right)$. The hypersingular boundary integral equations in (5.10) and (5.13) may be collocated over $0<\xi_{1}<L$ and $L_{0}<\xi_{1}<L_{0}+L$ respectively to generate $4\left(N_{0}+N_{1}\right)$ equations. For this purpose, two collocation points are chosen in the interior of each element. Specifically, on the element $E^{(m)}$, the two interior collocation points are given by

$$
\begin{aligned}
y_{1}^{(m)}=\frac{1}{4} x^{(m)}+\frac{3}{4} x^{(m+1)} \text { and } y_{2}^{(m)} & =\frac{3}{4} x^{(m)}+\frac{1}{4} x^{(m+1)} \\
(m & \left.=1,2, \cdots, 2 N_{0}+N_{1}\right) .
\end{aligned}
$$


Note that the elements involved in (5.10) are $E^{(1)}, E^{(2)}, \cdots, E^{\left(N_{0}+N_{1}-1\right)}$ and $E^{\left(N_{0}+N_{1}\right)}$ (the first $N_{0}+N_{1}$ elements), while those in (5.13) are $E^{\left(N_{0}+1\right)}, E^{\left(N_{0}+2\right)}$, $\cdots, E^{\left(2 N_{0}+N_{1}-1\right)}$ and $E^{\left(2 N_{0}+N_{1}\right)}$ (the last $N_{0}+N_{1}$ elements).

As before, the displacement jumps $\Delta u_{3 \mathrm{I}}^{(\mathrm{imp})}\left(x_{1}\right)$ and $\Delta u_{3 \mathrm{II}}^{(\mathrm{imp})}\left(x_{1}\right)$ are approximated using

$$
\begin{aligned}
\Delta u_{3 \mathrm{I}}^{(\mathrm{imp})}\left(x_{1}\right) \simeq & \sqrt{\left(x_{1}-a_{\mathrm{I}}^{(k)}\right)\left(b_{\mathrm{I}}^{(k)}-x_{1}\right)} \\
& \times \sum_{m=1}^{N_{\mathrm{I}}^{(k)}} \alpha_{\mathrm{I}}^{(k m)} U^{(m-1)}\left(\frac{2 x_{1}-b_{\mathrm{I}}^{(k)}-a_{\mathrm{I}}^{(k)}}{b_{\mathrm{I}}^{(k)}-a_{\mathrm{I}}^{(k)}}\right) \\
\text { for } a_{\mathrm{I}}^{(k)}< & x_{1}<b_{\mathrm{I}}^{(k)}(k=1,2, \ldots, M),
\end{aligned}
$$

and

$$
\begin{aligned}
\Delta u_{3 \mathrm{II}}^{(\mathrm{imp})}\left(x_{1}\right) \simeq & \sqrt{\left(x_{1}-a_{\mathrm{II}}^{(k)}\right)\left(b_{\mathrm{II}}^{(k)}-x_{1}\right)} \\
& \times \sum_{m=1}^{N_{\mathrm{II}}^{(k)}} \alpha_{\mathrm{II}}^{(k m)} U^{(m-1)}\left(\frac{2 x_{1}-b_{\mathrm{II}}^{(k)}-a_{\mathrm{II}}^{(k)}}{b_{\mathrm{II}}^{(k)}-a_{\mathrm{II}}^{(k)}}\right) \\
\text { for } a_{\mathrm{II}}^{(k)}< & x_{1}<b_{\mathrm{II}}^{(k)}(k=1,2, \ldots, M),
\end{aligned}
$$

where $\alpha_{\mathrm{I}}^{(k m)}$ and $\alpha_{\mathrm{II}}^{(k m)}$ are constants yet to be determined and $N_{\mathrm{I}}^{(k)}$ and $N_{\mathrm{II}}^{(k)}$ are positive integers.

There $\operatorname{are}\left(N_{\mathrm{I}}^{(1)}+N_{\mathrm{I}}^{(2)}+\cdots+N_{\mathrm{I}}^{(M)}+N_{\mathrm{II}}^{(1)}+N_{\mathrm{II}}^{(2)}+\cdots+N_{\mathrm{II}}^{(M)}\right)$ unknown constants in (5.28) and (5.29). The hypersingular boundary integral equations in (5.11) and (5.14) may be collocated over $a_{\mathrm{I}}^{(n)}<\xi_{1}<b_{\mathrm{I}}^{(n)}$ and $a_{\mathrm{II}}^{(n)}<\xi_{1}<b_{\mathrm{II}}^{(n)}$ to generate $\left(N_{\mathrm{I}}^{(1)}+N_{\mathrm{I}}^{(2)}+\cdots+N_{\mathrm{I}}^{(M)}+N_{\mathrm{II}}^{(1)}+N_{\mathrm{II}}^{(2)}+\cdots+N_{\mathrm{II}}^{(M)}\right)$ equations. Specifically, the collocation points over $a_{\mathrm{I}}^{(n)}<\xi_{1}<b_{\mathrm{I}}^{(n)}$ are given by

$$
\begin{aligned}
z_{\mathrm{I}}^{(n m)}= & \frac{a_{\mathrm{I}}^{(n)}+b_{\mathrm{I}}^{(n)}}{2}+\frac{b_{\mathrm{I}}^{(n)}-a_{\mathrm{I}}^{(n)}}{2} \cos \left(\frac{[2 m-1] \pi}{2 N_{\mathrm{I}}^{(n)}}\right) \\
& \text { for } m=1,2, \cdots, N_{\mathrm{I}}^{(n)}(n=1,2, \ldots, M),
\end{aligned}
$$


and those over $a_{\mathrm{II}}^{(n)}<\xi_{1}<b_{\mathrm{II}}^{(n)}$ by

$$
\begin{array}{r}
z_{\mathrm{II}}^{(n m)}=\frac{a_{\mathrm{II}}^{(n)}+b_{\mathrm{II}}^{(n)}}{2}+\frac{b_{\mathrm{II}}^{(n)}-a_{\mathrm{II}}^{(n)}}{2} \cos \left(\frac{[2 m-1] \pi}{2 N_{\mathrm{II}}^{(n)}}\right) \\
\quad \text { for } m=1,2, \cdots, N_{\mathrm{II}}^{(n)}(n=1,2, \ldots, M) .
\end{array}
$$

If the approximations (5.25), (5.26), (5.28) and (5.29) are substituted into (5.10), (5.11), (5.13) and (5.14), the hypersingular boundary integral equations (5.10) and (5.13) are collocated at $y_{i}^{(m)}(i=1,2)$ in (5.27) for $m=1,2, \cdots$, $N_{0}+N_{1}$ and $m=N_{0}+1, N_{0}+2, \cdots, 2 N_{0}+N_{1}$ respectively and (5.11) and (5.14) at the collocation points defined in (5.30) and (5.31) respectively, a system of linear algebraic equations can be set up for determining all the yet to be determined constant coefficients in (5.25), (5.26), (5.28) and (5.29). Once $\alpha_{\mathrm{I}}^{(\mathrm{km})}$ and $\alpha_{\mathrm{II}}^{(\mathrm{km})}$ are determined, (5.28) and (5.29) may be substituted into (5.6) to compute $k_{33 \mathrm{I}}$ and $k_{33 \mathrm{II}}$ approximately.

\subsubsection{Inplane Deformations}

For inplane deformations, the hypersingular boundary integro-differential equations (5.16), (5.17), (5.19) and (5.20) are approximated as algebraic equations as explained below.

From (5.24), the functions $u_{r}^{(\mathrm{imp})}\left(x_{1}, h^{ \pm} / 2\right)$ and $\sigma_{r 2}^{(\mathrm{imp})}\left(x_{1}, h^{ \pm} / 2\right)$ may be approximated over the first $N_{0}+N_{1}$ elements (in the interval $0 \leq x_{1} \leq L$ ) by

$$
\left.\begin{array}{l}
u_{r}^{(\mathrm{imp})}\left(x_{1}, h^{ \pm} / 2\right) \simeq x_{1} \phi_{r}^{(m)}+\psi_{r}^{(m)} \\
\sigma_{r 2}^{(\mathrm{mip})}\left(x_{1}, h^{ \pm} / 2\right) \simeq x_{1} \vartheta_{r}^{(m)}+\eta_{r}^{(m)}
\end{array}\right\} \text { over } E^{(m)}\left(m=1,2, \cdots, N_{0}+N_{1}\right)
$$

where $\phi_{r}^{(m)}, \psi_{r}^{(m)}, \vartheta_{r}^{(m)}$ and $\eta_{r}^{(m)}$ are constants yet to be determined. Over the last $N_{0}$ elements (in $\left.L \leq x_{1} \leq L+L_{0}\right)$, the functions $u_{r}^{(i m p)}\left(x_{1}, h^{ \pm} / 2\right)$ and 


\section{Micromechanical Models for Weak Interfaces}

$\sigma_{r 2}^{(\mathrm{imp})}\left(x_{1}, h^{ \pm} / 2\right)$ may be approximated by using

$$
\begin{aligned}
& \left.\begin{array}{l}
u_{r}^{(\mathrm{imp})}\left(x_{1}, h^{ \pm} / 2\right) \simeq\left(x_{1}-L\right) \phi_{r}^{(m)}+\psi_{r}^{(m)} \\
\sigma_{r 2}^{(\mathrm{imp})}\left(x_{1}, h^{ \pm} / 2\right) \simeq\left(x_{1}-L\right) \vartheta_{r}^{(m)}+\eta_{r}^{(m)}
\end{array}\right\} \text { over } E^{(m)} \\
& \left(m=N_{0}+N_{1}+1, N_{0}+N_{1}+2, \cdots, N_{0}+N_{1}+N_{0}\right) \text {. }
\end{aligned}
$$

The unknown displacement jumps $\Delta u_{r \mathrm{I}}^{(\mathrm{imp})}\left(x_{1}\right)$ and $\Delta u_{r \mathrm{II}}^{(\mathrm{imp})}\left(x_{1}\right)$ over interfaces I and II respectively may be approximated by using

$$
\begin{aligned}
\Delta u_{r \mathrm{I}}^{(\mathrm{imp})}\left(x_{1}\right) \simeq & \sqrt{\left(x_{1}-a_{\mathrm{I}}^{(k)}\right)\left(b_{\mathrm{I}}^{(k)}-x_{1}\right)} \\
& \times \sum_{m=1}^{N_{\mathrm{I}}^{(k)}} \alpha_{r \mathrm{I}}^{(k m)} U^{(m-1)}\left(\frac{2 x_{1}-b_{\mathrm{I}}^{(k)}-a_{\mathrm{I}}^{(k)}}{b_{\mathrm{I}}^{(k)}-a_{\mathrm{I}}^{(k)}}\right) \\
\text { for } a_{\mathrm{I}}^{(k)}< & x_{1}<b_{\mathrm{I}}^{(k)}(k=1,2, \ldots, M),
\end{aligned}
$$

and

$$
\begin{aligned}
\Delta u_{r \mathrm{II}}^{(\mathrm{imp})}\left(x_{1}\right) \simeq & \sqrt{\left(x_{1}-a_{\mathrm{II}}^{(k)}\right)\left(b_{\mathrm{II}}^{(k)}-x_{1}\right)} \\
& \times \sum_{m=1}^{N_{\mathrm{II}}^{(k)}} \alpha_{r \mathrm{II}}^{(k m)} U^{(m-1)}\left(\frac{2 x_{1}-b_{\mathrm{II}}^{(k)}-a_{\mathrm{II}}^{(k)}}{b_{\mathrm{II}}^{(k)}-a_{\mathrm{II}}^{(k)}}\right) \\
\text { for } a_{\mathrm{II}}^{(k)}< & x_{1}<b_{\mathrm{II}}^{(k)}(k=1,2, \ldots, M),
\end{aligned}
$$

where $\alpha_{r \mathrm{I}}^{(k m)}$ and $\alpha_{r \mathrm{II}}^{(k m)}$ are constants yet to be determined.

For two independent sets of external loads, if we substitute the approximations in (5.32), (5.33), (5.34) and (5.35) into hypersingular boundary integrodifferential equations (5.16), (5.17), (5.19) and (5.20), collocate (5.16) and (5.19) at $y_{i}^{(m)}(i=1,2)$ in (5.27) for $m=1,2, \cdots, N_{0}+N_{1}$ and $m=N_{0}+1$, $N_{0}+2, \cdots, 2 N_{0}+N_{1}$ respectively and collocate (5.17) and (5.20) at the relevant collocation points defined by (5.30) and (5.31) respectively, a system of linear algebraic equations may be set up to determine the unknown constants in (5.32), (5.33), (5.34) and (5.35). Once the displacement jumps over the 
micro-cracks on interfaces I and II are obtained, the effective stiffness coefficients $k_{r j \mathrm{I}}$ and $k_{r j \mathrm{II}}$ may be easily computed by using (5.8).

\subsection{Numerical Results for Antiplane Deforma- tions}

Numerical results are obtained here for some cases where the pair of microcracked interfaces undergo an antiplane deformation. The trimaterial is taken to be isotropic with elastic moduli $C_{44}^{(p)}=C_{55}^{(p)}=G^{(p)}(p=1,2,3)$, where $G^{(1)}$ and $G^{(3)}$ are the constant shear modulus of the half-spaces $x_{2}>h$ and $x_{2}<0$ respectively and $G^{(2)}$ is the constant shear modulus of the thin elastic layer.

\subsubsection{A Comparison of Two Micro-models}

Two sets of numerical values are obtained for the effective stiffness coefficients $k_{33 \mathrm{I}}$ and $k_{33 \mathrm{II}}$ as explained below.

The first set of numerical values is obtained by taking the micro-cracks on each interface to be of equal length and evenly distributed. The distance $L_{0}$ in Figure 5.1 is taken to be zero and a period length of each interface contains only one micro-crack $(M=1)$. Details of the micro-cracks on each of the interfaces are given below.

The micro-crack in the portion of interface I where $0<x_{1}<L, x_{2}=0$ has the length $2 a_{\mathrm{I}}$ and the tips $\left(a_{\mathrm{I}}^{(1)}, 0\right)$ and $\left(b_{\mathrm{I}}^{(1)}, 0\right)$ of the micro-crack are given by

$$
a_{\mathrm{I}}^{(1)}=\frac{L}{2}\left(1-\rho_{\mathrm{I}}\right), \quad b_{\mathrm{I}}^{(1)}=a_{\mathrm{I}}^{(1)}+2 a_{\mathrm{I}}
$$

The micro-cracks on the remaining parts of interface I are given by $a_{\mathrm{I}}^{(1)}+n L<$ $x_{1}<b_{\mathrm{I}}^{(1)}+n L$ for $n= \pm 1, \pm 2, \cdots$. 
Similarly, the micro-crack in the portion of interface II where $0<x_{1}<L$, $x_{2}=h$ has the length $2 a_{\mathrm{II}}$ and the tips $\left(a_{\mathrm{II}}^{(1)}, h\right)$ and $\left(b_{\mathrm{II}}^{(1)}, h\right)$ of the micro-crack are given by

$$
a_{\mathrm{II}}^{(1)}=\frac{L}{2}\left(1-\rho_{\mathrm{II}}\right), \quad b_{\mathrm{II}}^{(1)}=a_{\mathrm{II}}^{(1)}+2 a_{\mathrm{II}} .
$$

The micro-cracks on the remaining parts of interface II are given by $a_{\mathrm{II}}^{(1)}+n L<$ $x_{1}<b_{\mathrm{II}}^{(1)}+n L$ for $n= \pm 1, \pm 2, \cdots$. Note that $\rho_{\mathrm{I}}=2 a_{\mathrm{I}} / L$ and $\rho_{\mathrm{II}}=2 a_{\mathrm{II}} / L$. Refer to Figure 5.2 for a sketch of the periodic model here.

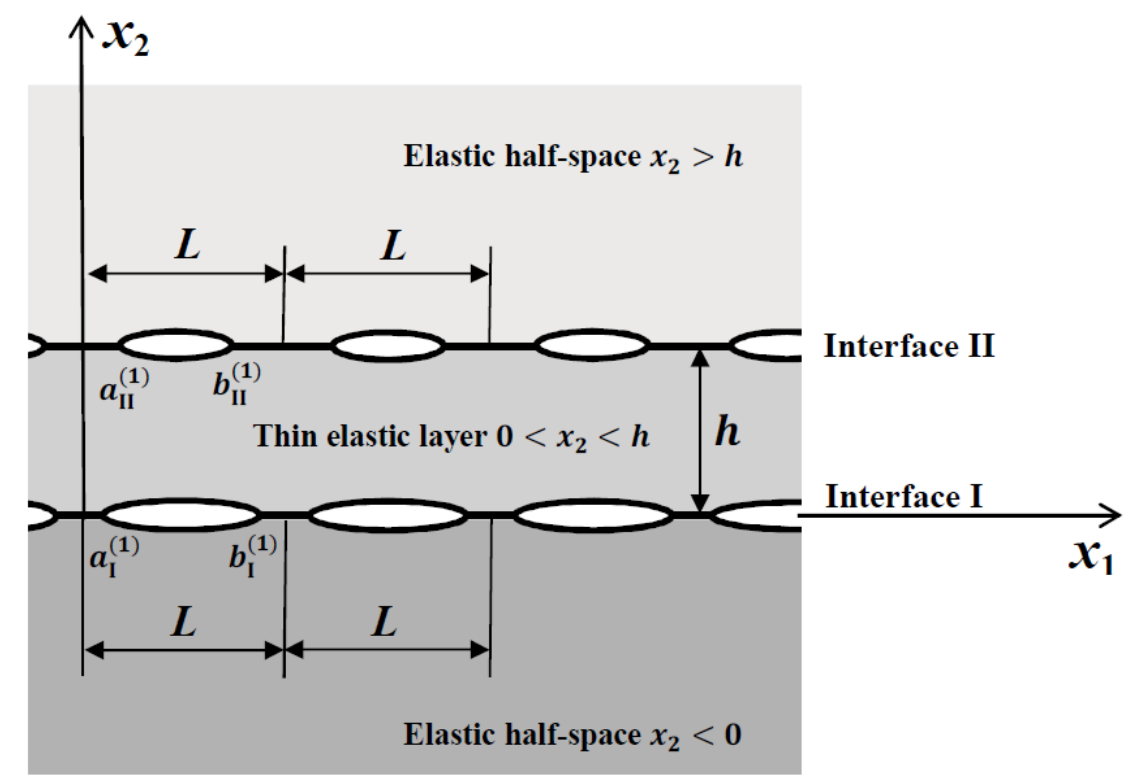

Figure 5.2: A geometrical sketch of the periodic model for interfaces I and II.

The hypersingular boundary integral equations for the periodic model are easily obtained by taking $M=1$ in (5.10), (5.11), (5.13) and (5.14). We solve (5.10), (5.11), (5.13) and (5.14) (for $M=1$ ) by using the numerical procedures given in Subsection 5.5.1 and estimate the effective stiffness coefficients $k_{33 \mathrm{I}}$ and $k_{33 \text { II }}$ by using (5.6).

The second set of numerical values for the antiplane effective stiffness coefficients are obtained by using a three-phase model for interfaces I and II. A 
geometrical sketch of the three-phase model is shown in Figure 5.3. Each of the interfaces is made up of three components: micro-cracked, perfectly bonded and effective regions. In Figure 5.3, all the three components are clearly shown in the region $0<x_{1}<L$ on interfaces I and II. The geometries of the interfaces are periodic with period $L$. The length of the effective region on each of the interfaces is assumed to be very large compared to the lengths of the micro-crack and the perfectly bonded part on the interface. Note that $c_{\mathrm{I}}^{(1)}=c_{\mathrm{I}}^{(2)}-d_{\mathrm{I}}^{(1)}$ and $c_{\mathrm{II}}^{(1)}=c_{\mathrm{II}}^{(2)}-d_{\mathrm{II}}^{(1)}$.

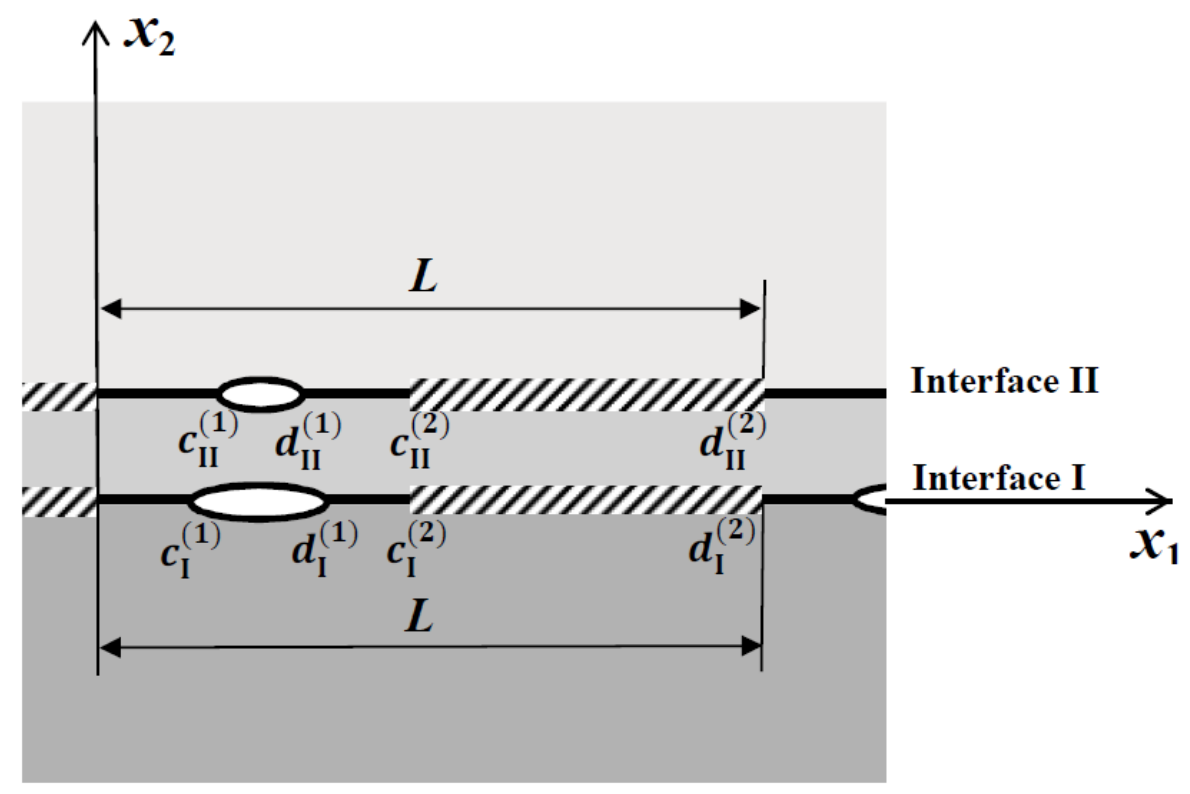

Figure 5.3: A geometrical sketch of the three-phase model for interfaces I and II.

For the three-phase model, the damage ratios for interfaces I and II corresponding to (5.2) are respectively given by

$$
\rho_{\mathrm{I}}=\frac{d_{\mathrm{I}}^{(1)}-c_{\mathrm{I}}^{(1)}}{c_{\mathrm{I}}^{(2)}} \text { and } \rho_{\mathrm{II}}=\frac{d_{\mathrm{II}}^{(1)}-c_{\mathrm{II}}^{(1)}}{c_{\mathrm{II}}^{(2)}} .
$$

As in Section 5.3, the displacement $u_{3}$ and the stress $\sigma_{32}$ may be written into 


\section{Micromechanical Models for Weak Interfaces}

the form in (3.5), where $u_{3}^{\text {(ext) }}$ and $\sigma_{32}^{\text {(ext) }}$ are respectively the displacement and the stress fields in the trimaterial for the corresponding case where the entire interfaces I and II are perfectly bonded, and $u_{3}^{(\mathrm{imp})}$ and $\sigma_{32}^{(\mathrm{imp})}$ are respectively the displacement and stress due to the interaction between the micro-cracks and the effective regions. As before, the prescribed stress fields $\sigma_{32}^{(\text {ext })}\left(x_{1}, 0\right)$ and $\sigma_{32}^{(\text {ext })}\left(x_{1}, h\right)$ are given in (5.9).

The interfacial conditions for the three-phase model are given by

$$
\begin{aligned}
& \sigma_{32}^{(\mathrm{imp})}\left(x_{1}, 0^{ \pm}\right)=-\sigma_{3} \text { for }\left(x_{1}, 0\right) \in D_{\mathrm{I}}, \\
& \sigma_{32}^{(\mathrm{imp})}\left(x_{1}, h^{ \pm}\right)=-\sigma_{3} \text { for }\left(x_{1}, h\right) \in D_{\text {II }} \text {, } \\
& \left.\begin{array}{rl}
\Delta u_{3 \mathrm{I}}^{(\mathrm{imp})}\left(x_{1}\right) & =0 \\
\sigma_{32}^{(\mathrm{imp})}\left(x_{1}, 0^{+}\right) & =\sigma_{32}^{(\mathrm{imp})}\left(x_{1}, 0^{-}\right)
\end{array}\right\} \text {for }\left(x_{1}, 0\right) \in P_{\mathrm{I}}, \\
& \left.\begin{array}{rl}
\Delta u_{3 I \mathrm{I}}^{(\mathrm{imp})}\left(x_{1}\right) & =0 \\
\sigma_{32}^{(\mathrm{imp})}\left(x_{1}, h^{+}\right) & =\sigma_{32}^{(\mathrm{imp})}\left(x_{1}, h^{-}\right)
\end{array}\right\} \text {for }\left(x_{1}, h\right) \in P_{\mathrm{II}}, \\
& k_{33 \mathrm{I}} \Delta u_{3 \mathrm{I}}^{(\mathrm{imp})}\left(x_{1}\right)=\sigma_{3}+\sigma_{32}^{(\mathrm{imp})}\left(x_{1}, 0^{ \pm}\right) \text {for }\left(x_{1}, 0\right) \in E_{\mathrm{I}} \text {, } \\
& k_{33 \mathrm{II}} \Delta u_{3 \mathrm{II}}^{(\mathrm{imp})}\left(x_{1}\right)=\sigma_{3}+\sigma_{32}^{(\mathrm{imp})}\left(x_{1}, h^{ \pm}\right) \text {for }\left(x_{1}, h\right) \in E_{\mathrm{II}} \text {, }
\end{aligned}
$$

where $D_{\mathrm{I}}, D_{\mathrm{II}}, P_{\mathrm{I}}$ and $P_{\mathrm{II}}$ are defined below (5.5) and $E_{\mathrm{I}}$ and $E_{\mathrm{II}}$ denote the effective regions on interfaces I and II respectively.

The effective regions $E_{\mathrm{I}}$ and $E_{\mathrm{II}}$ behave according to the spring-like interface model in (5.4) but with unknown (yet to determined) effective stiffness $k_{33 \mathrm{I}}$ and $k_{33 I I}$. As $k_{33 \text { I }}$ and $k_{33 I I}$ in (5.39) are unknown coefficients, two more equations are needed to complete the formulation for the three-phase model. They are given by

$$
\begin{gathered}
k_{33 \mathrm{I}} \int_{c_{\mathrm{I}}^{(1)}}^{d_{\mathrm{I}}^{(1)}} \Delta u_{3 \mathrm{I}}^{(\mathrm{imp})}\left(x_{1}\right) d x_{1}=c_{\mathrm{I}}^{(2)} \sigma_{3}, \\
k_{33 \mathrm{II}} \int_{c_{\mathrm{II}}^{(1)}}^{d_{\mathrm{II}}^{(1)}} \Delta u_{3 \mathrm{II}}^{(\mathrm{imp})}\left(x_{1}\right) d x_{1}=c_{\mathrm{II}}^{(2)} \sigma_{3} .
\end{gathered}
$$


As in the analysis in Subsection 5.4.1, the trimaterial in Figure 5.3 is divided into the subdomains $x_{2}<h / 2$ and $x_{2}>h / 2$ to derive hypersingular boundary integral equations for the three-phase model.

From the boundary integral equations for the lower subdomain $x_{2}<h / 2$, the hypersingular integral equations for the stress $\sigma_{32}^{(\mathrm{imp})}$ on the artificial boundary $x_{2}=h^{-} / 2$ and interface I (where $x_{2}=0^{+}$) are respectively given by

$$
\begin{aligned}
& \frac{1}{2} \sigma_{32}^{(\mathrm{imp})}\left(\xi_{1}, h^{-} / 2\right) \\
= & -\frac{1}{2 \pi} f_{0}^{L} u_{3}^{(\mathrm{imp})}\left(x_{1}, h^{-} / 2\right)\left[\frac{1}{\left(x_{1}-\xi_{1}\right)^{2}}+\Theta\left(x_{1}, \xi_{1}\right)\right] d x_{1} \\
& +\frac{1}{2 \pi} \frac{\beta^{(2)}-\beta^{(3)}}{\beta^{(2)}+\beta^{(3)}} \int_{0}^{L} u_{3}^{(\mathrm{imp})}\left(x_{1}, h^{-} / 2\right) \operatorname{Re}\left\{\Omega\left(x_{1}, \xi_{1},\left(\tau^{(2)}-\bar{\tau}^{(2)}\right) h^{-} / 2\right)\right\} d x_{1} \\
& -\frac{\beta^{(2)}-\beta^{(3)}}{2 \pi\left(\beta^{(2)}+\beta^{(3)}\right)} \int_{0}^{L} \sigma_{32}^{(\mathrm{imp})}\left(x_{1}, h^{-} / 2\right) \operatorname{Re}\left\{\Gamma_{0}\left(x_{1}, \xi_{1},\left(\tau^{(2)}-\bar{\tau}^{(2)}\right) h^{-} / 2\right)\right\} d x_{1} \\
& +\frac{\beta^{(3)}}{\pi\left(\beta^{(2)}+\beta^{(3)}\right)} \sum_{k=1}^{2} \int_{c_{\mathrm{I}}^{(k)}}^{d_{\mathrm{I}}^{(k)}} \Delta u_{3 \mathrm{I}}^{(\mathrm{imp})}\left(x_{1}\right) \operatorname{Re}\left\{\Omega\left(x_{1}, \xi_{1},-\tau^{(2)} h^{-} / 2\right)\right\} d x_{1} \\
& \text { for } 0<\xi_{1}<L,
\end{aligned}
$$

and

$$
\begin{aligned}
& \int_{0}^{L} u_{3}^{(\mathrm{imp})}\left(x_{1}, h^{-} / 2\right) \operatorname{Re}\left\{\Omega\left(x_{1}, \xi_{1}, \tau^{(2)} h^{-} / 2\right)\right\} d x_{1} \\
& -\int_{0}^{L} \sigma_{32}^{(\mathrm{imp})}\left(x_{1}, h^{-} / 2\right) \operatorname{Re}\left\{\Gamma_{0}\left(x_{1}, \xi_{1}, \tau^{(2)} h^{-} / 2\right)\right\} d x_{1} \\
& -\int_{c_{\mathrm{I}}^{(n)}}^{d_{\mathrm{I}}^{(n)}} \frac{\Delta u_{3 \mathrm{I}}^{(\mathrm{imp})}\left(x_{1}\right)}{\left(x_{1}-\xi_{1}\right)^{2}} d x_{1} \\
& -\sum_{k=1}^{2} \int_{c_{\mathrm{I}}^{(k)}}^{d_{\mathrm{I}}^{(k)}} \frac{\Delta u_{3 \mathrm{I}}^{(\mathrm{imp})}\left(x_{1}\right)}{\left(x_{1}-\xi_{1}\right)^{2}} d x_{1} \\
& -\sum_{k=1}^{2} \int_{c_{\mathrm{I}}^{(k)}}^{d_{\mathrm{I}}^{(k)}} \Delta u_{3 \mathrm{I}}^{(\mathrm{imp})}\left(x_{1}\right) \Theta\left(x_{1}, \xi_{1}\right) d x_{1}
\end{aligned}
$$




$$
\begin{aligned}
= & \frac{\pi\left(\beta^{(2)}+\beta^{(3)}\right)\left(\sigma_{3}-\delta^{(n 2)} k_{33 \mathrm{I}} \Delta u_{3 \mathrm{I}}^{(\mathrm{imp})}\left(\xi_{1}\right)\right)}{\beta^{(3)}} \\
& \text { for } c_{\mathrm{I}}^{(n)}<\xi_{1}<d_{\mathrm{I}}^{(n)}(n=1,2),
\end{aligned}
$$

where $\delta^{(n 2)}$ is such that $\delta^{(12)}=0$ and $\delta^{(22)}=1$.

From the boundary integral equations for the upper subdomain $x_{2}>h / 2$, the hypersingular integral equations for the stress $\sigma_{32}^{(\mathrm{imp})}$ on the artificial boundary $x_{2}=h^{+} / 2$ and interface II (where $\left.x_{2}=h^{-}\right)$are

$$
\begin{aligned}
& \frac{1}{2} \sigma_{32}^{(\mathrm{imp})}\left(\xi_{1}, h^{+} / 2\right) \\
= & \frac{1}{2 \pi} \int_{0}^{L} u_{3}^{(\mathrm{imp})}\left(x_{1}, h^{+} / 2\right)\left[\frac{1}{\left(x_{1}-\xi_{1}\right)^{2}}+\Theta\left(x_{1}, \xi_{1}\right)\right] d x_{1} \\
& -\frac{\beta^{(2)}-\beta^{(1)}}{2 \pi\left(\beta^{(2)}+\beta^{(1)}\right)} \int_{0}^{L} u_{3}^{(\mathrm{imp})}\left(x_{1}, h^{+} / 2\right) \\
& \times \operatorname{Re}\left\{\Omega\left(x_{1}, \xi_{1},-\left(\tau^{(2)}-\bar{\tau}^{(2)}\right) h^{+} / 2\right)\right\} d x_{1} \\
& +\frac{\beta^{(2)}-\beta^{(1)}}{2 \pi\left(\beta^{(2)}+\beta^{(1)}\right)} \int_{0}^{L} \sigma_{32}^{(\mathrm{imp})}\left(x_{1}, h^{+} / 2\right) \\
& \times \operatorname{Re}\left\{\Gamma_{0}\left(x_{1}, \xi_{1},-\left(\tau^{(2)}-\bar{\tau}^{(2)}\right) h^{+} / 2\right)\right\} d x_{1} \\
& +\frac{\beta^{(1)}}{\pi\left(\beta^{(2)}+\beta^{(1)}\right)} \sum_{k=1}^{2} \int_{c_{\mathrm{II}}^{(k)}}^{d_{\mathrm{II}}^{(k)}} \Delta u_{3 \mathrm{II}}^{(\mathrm{imp})}\left(x_{1}\right) \operatorname{Re}\left\{\Omega\left(x_{1}, \xi_{1}, \tau^{(2)} h^{+} / 2\right)\right\} d x_{1} \\
& \text { for } 0<\xi_{1}<L,
\end{aligned}
$$

and

$$
\begin{aligned}
& \int_{0}^{L} u_{3}^{(\mathrm{imp})}\left(x_{1}, h^{+} / 2\right) \operatorname{Re}\left\{\Omega\left(x_{1}, \xi_{1},-\tau^{(2)} h^{-} / 2\right)\right\} d x_{1} \\
& -\int_{0}^{L} \sigma_{32}^{(\mathrm{imp})}\left(x_{1}, h^{+} / 2\right) \operatorname{Re}\left\{\Gamma_{0}\left(x_{1}, \xi_{1},-\tau^{(2)} h^{-} / 2\right)\right\} d x_{1} \\
& +f_{c_{\mathrm{II}}^{(n)}}^{d_{\mathrm{II}}^{(n)}} \frac{\Delta u_{3 \mathrm{II}}^{(\mathrm{imp})}\left(x_{1}\right)}{\left(x_{1}-\xi_{1}\right)^{2}} d x_{1}
\end{aligned}
$$




$$
\begin{aligned}
& +\sum_{\substack{k=1 \\
k \neq n}}^{2} \int_{c_{\mathrm{II}}}^{(k)} d_{\mathrm{II}}^{(k)} \frac{\Delta u_{3 \mathrm{II}}^{(\mathrm{imp})}\left(x_{1}\right)}{\left(x_{1}-\xi_{1}\right)^{2}} d x_{1} \\
& +\sum_{\substack{k=1 \\
c_{\mathrm{II}}}}^{d_{\mathrm{II}}^{(k)}} \Delta u_{3 \mathrm{II}}^{(\mathrm{imp})}\left(x_{1}\right) \Theta\left(x_{1}, \xi_{1}\right) d x_{1} \\
= & \frac{\pi\left(\beta^{(2)}+\beta^{(1)}\right)\left(-\sigma_{3}+\delta^{(n 2)} k_{33 \mathrm{II}} \Delta u_{3 \mathrm{II}}^{(\mathrm{imp})}\left(\xi_{1}\right)\right)}{\beta^{(1)}} \\
& \text { for } c_{\mathrm{II}}^{(n)}<\xi_{1}<d_{\mathrm{II}}^{(n)}(n=1,2) .
\end{aligned}
$$

The hypersingular boundary integral equations (5.41), (5.42), (5.43) and (5.44) are to be solved numerically together with (5.40) for the unknowns $\Delta u_{3 \mathrm{I}}^{(\mathrm{imp})}\left(x_{1}\right), \Delta u_{3 \mathrm{II}}^{(\mathrm{imp})}\left(x_{1}\right), u_{3}^{(\mathrm{imp})}\left(x_{1}, h^{ \pm} / 2\right), \sigma_{32}^{(\mathrm{imp})}\left(x_{1}, h^{ \pm} / 2\right)$ and $k_{33 \mathrm{I}}$ and $k_{33 \mathrm{II}}$.

For the approximation of $u_{3}^{\text {(imp) }}\left(x_{1}, h^{-} / 2\right)$ and $\sigma_{32}^{\text {(imp) }}\left(x_{1}, h^{-} / 2\right)$, the interval $0 \leq x_{1} \leq L$ is discretized into elements as described in Section 5.5 and (5.25) and (5.26) are still valid here. As in (5.28) and (5.29), the displacement jumps $\Delta u_{3 \mathrm{I}}^{(\mathrm{imp})}\left(x_{1}\right)$ and $\Delta u_{3 \mathrm{II}}^{(\mathrm{imp})}\left(x_{1}\right)$ over the micro-cracks $c_{\mathrm{I}}^{(1)}<x_{1}<d_{\mathrm{I}}^{(1)}, x_{2}=0$ (interface I) and $c_{\mathrm{II}}^{(1)}<x_{1}<d_{\mathrm{II}}^{(1)}, x_{2}=h$ (interface II) are given by

$$
\begin{aligned}
\Delta u_{3 \mathrm{I}}^{(\mathrm{imp})}\left(x_{1}\right) \simeq & \sqrt{\left(x_{1}-c_{\mathrm{I}}^{(1)}\right)\left(d_{\mathrm{I}}^{(1)}-x_{1}\right)} \\
& \times \sum_{m=1}^{N_{\mathrm{I}}} \alpha_{\mathrm{I}}^{(m)} U^{(m-1)}\left(\frac{2 x_{1}-d_{\mathrm{I}}^{(1)}-c_{\mathrm{I}}^{(1)}}{d_{\mathrm{I}}^{(1)}-c_{\mathrm{I}}^{(1)}}\right) \\
& \text { for } c_{\mathrm{I}}^{(1)}<x_{1}<d_{\mathrm{I}}^{(1)},
\end{aligned}
$$

and

$$
\begin{aligned}
\Delta u_{3 \mathrm{II}}^{(\mathrm{imp})}\left(x_{1}\right) \simeq & \sqrt{\left(x_{1}-c_{\mathrm{II}}^{(1)}\right)\left(d_{\mathrm{II}}^{(1)}-x_{1}\right)} \\
& \times \sum_{m=1}^{N_{\mathrm{II}}} \alpha_{\mathrm{II}}^{(m)} U^{(m-1)}\left(\frac{2 x_{1}-d_{\mathrm{II}}^{(1)}-c_{\mathrm{II}}^{(1)}}{d_{\mathrm{II}}^{(1)}-c_{\mathrm{II}}^{(1)}}\right) \\
& \text { for } c_{\mathrm{II}}^{(1)}<x_{1}<d_{\mathrm{II}}^{(1)} .
\end{aligned}
$$

For approximating the unknown function $\Delta u_{3 \mathrm{I}}^{(\mathrm{imp})}\left(x_{1}\right)$ over the effective region on interface I, the interval $c_{\mathrm{I}}^{(2)}<x_{1}<d_{\mathrm{I}}^{(2)}$ is discretized into $N_{2}$ equal 
length elements given by $E_{\mathrm{I}}^{(1)}, E_{\mathrm{I}}^{(2)}, \cdots, E_{\mathrm{I}}^{\left(N_{2}-1\right)}$ and $E_{\mathrm{I}}^{\left(N_{2}\right)}$. Similarly, for $\Delta u_{3 \mathrm{II}}^{(\mathrm{imp})}\left(x_{1}\right)$ over the effective region on interface II, the interval $c_{\mathrm{II}}^{(2)}<x_{1}<d_{\mathrm{II}}^{(2)}$ is discretized into $N_{3}$ equal length elements given by $E_{\mathrm{II}}^{(1)}, E_{\mathrm{II}}^{(2)}, \cdots, E_{\mathrm{II}}^{\left(N_{3}-1\right)}$ and $E_{\mathrm{II}}^{\left(N_{3}\right)}$. The displacement jumps $\Delta u_{3 \mathrm{I}}^{(\mathrm{imp})}\left(x_{1}\right)$ and $\Delta u_{3 \mathrm{II}}^{(\mathrm{imp})}\left(x_{1}\right)$ over the effective regions on interfaces I and II respectively are approximated using

$$
\Delta u_{3 \mathrm{I}}^{(\mathrm{imp})}\left(x_{1}\right) \simeq x_{1} \omega_{\mathrm{I}}^{(m)}+\gamma_{\mathrm{I}}^{(m)} \text { over } E_{\mathrm{I}}^{(m)}\left(m=1,2, \cdots, N_{2}\right),
$$

and

$$
\Delta u_{3 \mathrm{II}}^{(\mathrm{imp})}\left(x_{1}\right) \simeq x_{1} \omega_{\mathrm{II}}^{(m)}+\gamma_{\mathrm{II}}^{(m)} \text { over } E_{\mathrm{II}}^{(m)}\left(m=1,2, \cdots, N_{3}\right),
$$

where $\omega_{\mathrm{I}}^{(p)}, \gamma_{\mathrm{I}}^{(p)}, \omega_{\mathrm{II}}^{(p)}$ and $\gamma_{\mathrm{II}}^{(p)}$ are constants yet to be determined.

The hypersingular boundary integral equations (5.41), (5.42), (5.43) and (5.44) may be discretized and collocated as explained in Subsection 5.5.1 to obtain a system of linear algebraic equations with the unknown effective stiffness coefficients $k_{33 \mathrm{I}}$ and $k_{33 \mathrm{II}}$ and coefficients in the approximations of the unknown functions in the integral equations. However, the discretization of the two equations in (5.40) gives rise to a pair of quadratic equations in the unknown constants. The algebraic equations may be solved numerically by using an iterative method as described in Subsection 3.5.1.

The numerical values of the antiplane effective stiffness coefficients obtained using the periodic model involving evenly distributed micro-cracks of equal length are compared here with the corresponding values computed by the threephase model. The two sets of values are expected to be close to each other.

For $G^{(1)} / G^{(2)}=G^{(3)} / G^{(2)}=2$, we plot and compare the two sets of the nondimensionalized effective stiffness coefficient $a_{\mathrm{I}} k_{33 \mathrm{I}} / G^{(2)}$ against $a_{\mathrm{I}} / h$ for $\rho_{\mathrm{I}}=$ $\rho_{\mathrm{II}}=0.3,0.5$ and 0.7 in Figure 5.4. Note that here $a_{\mathrm{I}} k_{33 \mathrm{I}} / G^{(2)}=a_{\mathrm{II}} k_{33 \mathrm{II}} / G^{(2)}$. The plots obtained by the numerical procedures for the periodic model are 


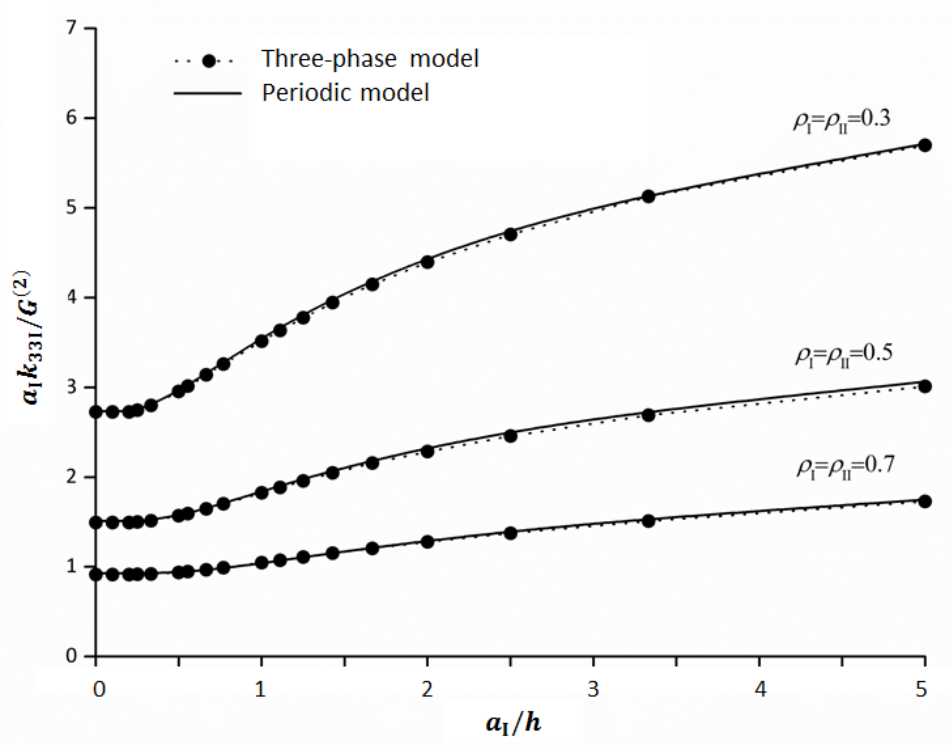

Figure 5.4: Plots of $a_{\mathrm{I}} k_{33 \mathrm{I}} / G^{(2)}$ against $a_{\mathrm{I}} / h$ for $G^{(1)} / G^{(2)}=G^{(3)} / G^{(2)}=2$ and $\rho_{\mathrm{I}}=\rho_{\mathrm{II}}=0.3,0.5$ and 0.7 .

close to the ones from the corresponding three-phase model. For a fixed $\rho_{\mathrm{I}}$ in Figure 5.4, the percentage difference between the values of $a_{\mathrm{I}} k_{33 \mathrm{I}} / G^{(2)}$ for the two models for a selected $a_{\mathrm{I}} / h$ is less than $1.6 \%$.

\subsubsection{Micromechanical-statistical Simulations}

The statistical approach in Subsections 3.4.3 and 4.2.3 is used to randomly generate the micro-cracks on each of the interfaces. The lengths of the $M$ micro-cracks (within a period length of each of the interfaces) are randomly generated to follow a chi-square distribution of degree of freedom $k$, which is denoted by $\chi^{2}(k)$. In general, the micro-crack length distribution on interface I may be different from that on interface II. The distance $L_{0}$ in Figure 5.1 is a randomly generated real number within the range $0 \leq L_{0}<L$. The micro-cracks are positioned randomly over the period intervals $0<x_{1}<L$ 


\section{Micromechanical Models for Weak Interfaces}

and $L_{0}<x_{1}<L_{0}+L$ on interfaces I and II respectively.

For fixed values of the shear modulus $G^{(p)}(p=1,2,3), \widehat{a}_{\mathrm{I}} / h\left(\widehat{a}_{\mathrm{I}}\right.$ is the average half length of the micro-cracks on interface I), number of micro-cracks on each interface and the damage ratios $\rho_{\mathrm{I}}$ and $\rho_{\mathrm{II}}$ in (5.2), $N$ pairs of microcracked interfaces are generated randomly as described above for estimating the effective stiffness of interfaces I and II. (Note that all the interfaces contain the same number of micro-cracks per period interval of the interface.) For each pair of the interfaces, the non-dimensionalized effective stiffness coefficients $\widehat{a}_{\mathrm{I}} k_{33 \mathrm{I}} / G^{(2)}$ and $\widehat{a}_{\mathrm{II}} k_{33 \mathrm{II}} / G^{(2)}$ are calculated as explained in Section 5.1.

If $\widehat{a}_{\mathrm{I}} k_{33 \mathrm{I}} / G^{(2)}$ and $\widehat{a}_{\mathrm{II}} k_{33 \mathrm{II}} / G^{(2)}$ of the $n$-th pair of interfaces are respectively denoted as $K_{33 \mathrm{I}}^{(n)}$ and $K_{33 \mathrm{II}}^{(n)}(n=1,2, \cdots, N)$, then the mean value $\widehat{K}_{33 \mathrm{I}}$ and the standard deviation $s_{33 \text { I }}$ of the non-dimensionalized effective stiffness coefficient $K_{33 \mathrm{II}}^{(n)}$ are given by

$$
\widehat{K}_{33 \mathrm{I}}=\frac{1}{N} \sum_{n=1}^{N} K_{33 \mathrm{I}}^{(n)} \text { and } s_{33 \mathrm{I}}=\sqrt{\frac{1}{N-1} \sum_{n=1}^{N}\left(K_{33 \mathrm{I}}^{(n)}-\widehat{K}_{33 \mathrm{I}}\right)^{2}} \text {, }
$$

and the mean value $\widehat{K}_{33 I I}$ and the corresponding standard deviation $s_{33 I I}$ of the non-dimensionalized effective stiffness coefficient $K_{33 I I}^{(n)}$ are given by

$$
\widehat{K}_{33 \mathrm{II}}=\frac{1}{N} \sum_{n=1}^{N} K_{33 \mathrm{II}}^{(n)} \text { and } s_{33 \mathrm{II}}=\sqrt{\frac{1}{N-1} \sum_{n=1}^{N}\left(K_{33 \mathrm{II}}^{(n)}-\widehat{K}_{33 \mathrm{II}}\right)^{2}} .
$$

The number of micro-cracks required on a period interval of each interface for homogenizing the effective stiffness coefficients of the interface is examined as in Subsections 3.7.1 and 4.6.1. As before, our investigations show that at least 40 micro-cracks per period interval of each interface are required for homogenizing the effective stiffness coefficients of the interface. Thus, for our 


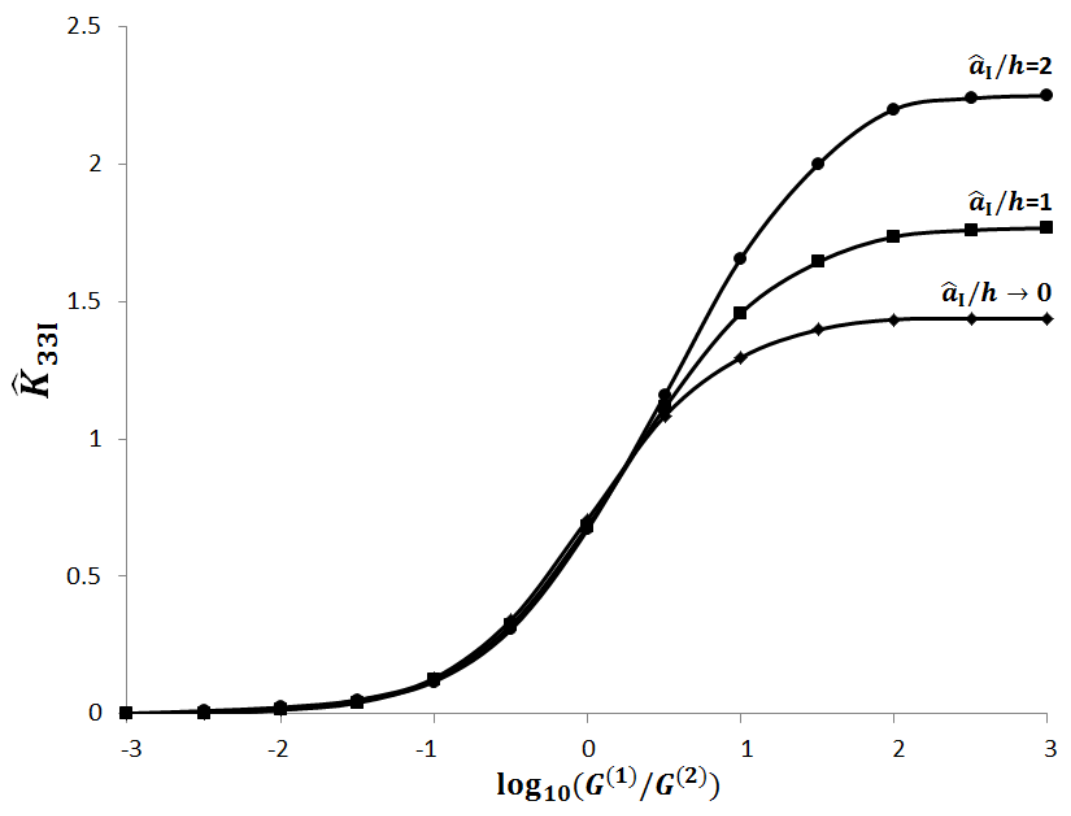

Figure 5.5: Plots of $\widehat{K}_{33 \mathrm{I}}$ against $\log _{10}\left(G^{(1)} / G^{(2)}\right)$ for $\rho_{\mathrm{I}}=\rho_{\mathrm{II}}=0.5, G^{(1)} / G^{(2)}$ $=G^{(3)} / G^{(2)}$ and selected values of $\widehat{a}_{\mathrm{I}} / h$.

statistical simulations here, we employ 40 micro-cracks per period interval on each of the interfaces.

The effects of the elastic moduli, the width of the layer and the damage ratios on the effective stiffness coefficients $\widehat{K}_{33 \text { I }}$ and $\widehat{K}_{33 I}$ are examined as follows. For fixed values of $\rho_{\mathrm{I}}, \rho_{\mathrm{II}}, \widehat{a}_{\mathrm{I}} / h, G^{(1)} / G^{(2)}$ and $G^{(3)} / G^{(2)}, 50$ pairs of interfaces are randomly generated for the statistical simulations. For each pair of interfaces, the number of the micro-cracks over a period interval of each interface is taken to be 40 . For each interface, the lengths of the micro-cracks are randomly generated by using the $\chi^{2}(5)$ distribution.

For $\rho_{\mathrm{I}}=\rho_{\mathrm{II}}=0.5$ and $G^{(1)} / G^{(2)}=G^{(3)} / G^{(2)}$, Figure 5.5 plots the means $\widehat{K}_{33 \text { I }}$ of the non-dimensionalized effective stiffness against $\log _{10}\left(G^{(1)} / G^{(2)}\right)$ for selected values of $\widehat{a}_{\mathrm{I}} / h$. As expected, the values of $\widehat{K}_{33 \mathrm{II}}$ are observed to be very close to $\widehat{K}_{33 \mathrm{I}}$. For larger values of $G^{(1)} / G^{(2)}$ within the range $0.5 \leq$ 


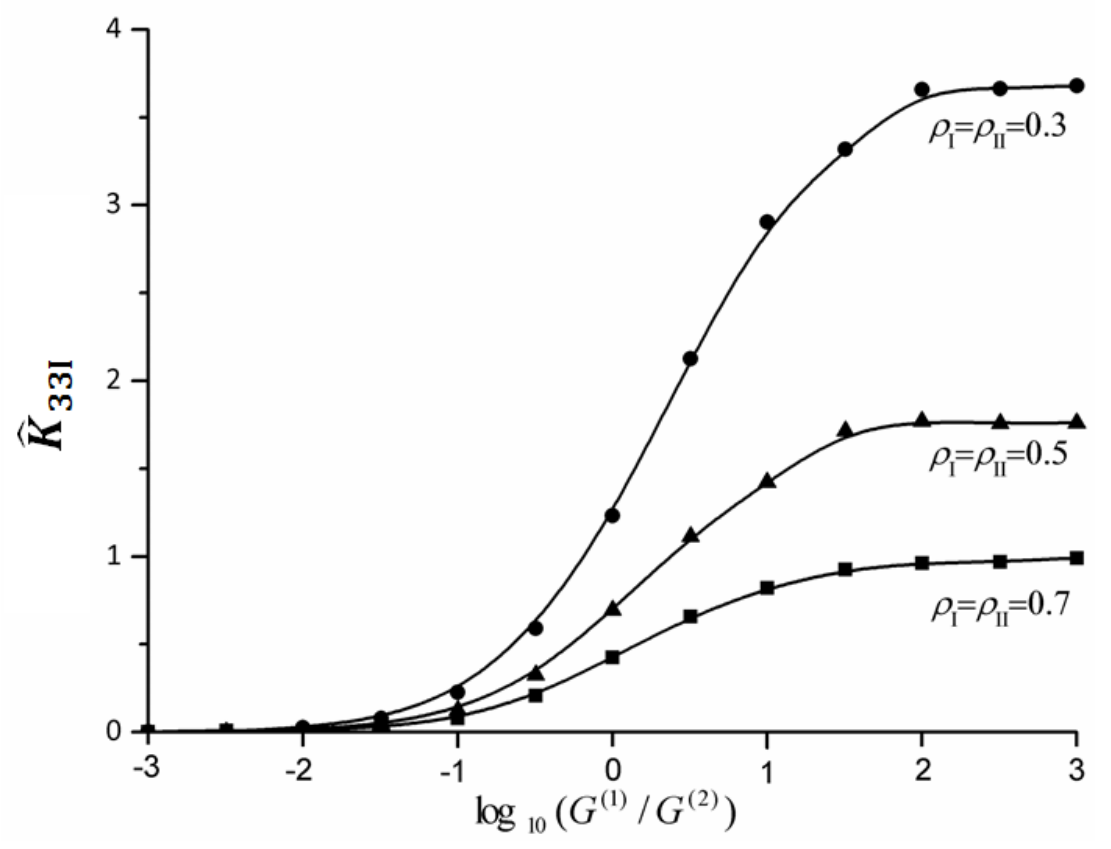

Figure 5.6: Plots of $\widehat{K}_{33 \mathrm{I}}$ against $\log _{10}\left(G^{(1)} / G^{(2)}\right)$ for $\widehat{a}_{\mathrm{I}} / h=1, G^{(1)} / G^{(2)}=$ $G^{(3)} / G^{(2)}, \rho_{\mathrm{I}}=\rho_{\mathrm{II}}=0.3,0.5$ and 0.7 .

$\log _{10}\left(G^{(1)} / G^{(2)}\right) \leq 3$, the plots of the non-dimensionalized effective stiffness for the different values of $\widehat{a}_{\mathrm{I}} / h$ are visually distinguishable. For a selected $G^{(1)} / G^{(2)}$ such that $0.5 \leq \log _{10}\left(G^{(1)} / G^{(2)}\right) \leq 3$, the value of $\widehat{K}_{33 \mathrm{I}}$ is larger for larger $\widehat{a}_{\mathrm{I}} / h$. For $G^{(1)} / G^{(2)}>100, \widehat{K}_{33 \mathrm{I}}$ increases very slowly with increasing $G^{(1)} / G^{(2)}$. For a fixed value of $\widehat{a}_{\mathrm{I}} / h$, it appears that $\widehat{K}_{33 \mathrm{I}}$ tends to a constant as $G^{(1)} / G^{(2)}$ increases. The width of the thin layer seems to have an obvious effect on the effective stiffness only if the elastic material for the thin layer is soft enough compared to the materials in the two half-spaces. As $G^{(1)} / G^{(2)} \rightarrow 0^{+}$, the values of $\widehat{K}_{33 \mathrm{I}}$ tend to zero, that is, if the thin layer is extremely hard relative to the two half-spaces, the magnitudes of the effective stiffness for both interfaces I and II may be very small.

In Figure 5.6, $\widehat{K}_{33 \mathrm{I}}$ is plotted against $\log _{10}\left(G^{(1)} / G^{(2)}\right)$ for $\widehat{a}_{\mathrm{I}} / h=1, G^{(1)} / G^{(2)}$ 
$=G^{(3)} / G^{(2)}, \rho_{\mathrm{I}}=\rho_{\mathrm{II}}=0.3,0.5$ and 0.7 . Values of $\widehat{K}_{33 \mathrm{II}}$ are very close to $\widehat{K}_{33 \mathrm{I}}$. It is observed that the non-dimensionalized effective stiffness $\widehat{K}_{33 \mathrm{I}}$ is larger when the two interfaces have smaller damage ratios. This is not surprising as the micro-cracks are more stable and hence the displacement jumps over the micro-cracks are smaller when the micro-cracks are less densely located on the interface.

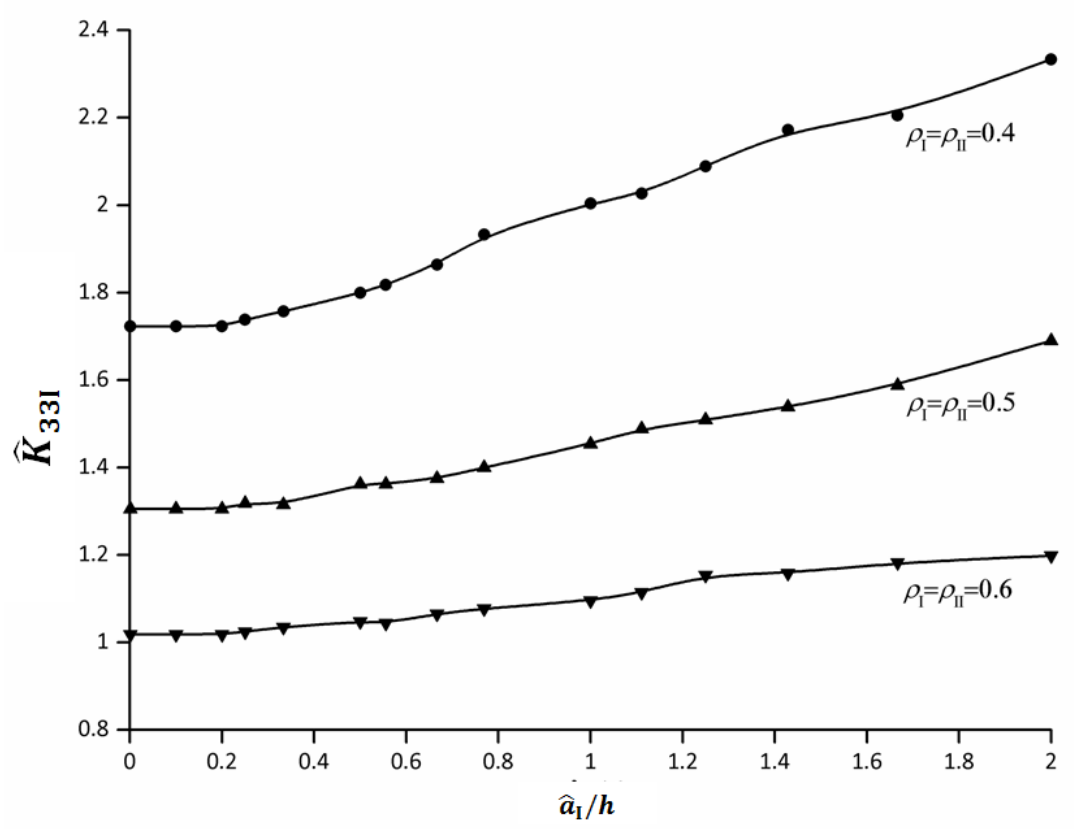

Figure 5.7: Plots of $\widehat{K}_{33 \mathrm{I}}$ against $\widehat{a}_{\mathrm{I}} / h$ for $G^{(1)} / G^{(2)}=G^{(3)} / G^{(2)}=10, \rho_{\mathrm{I}}=$ $\rho_{\mathrm{II}}=0.4,0.5$ and 0.6 .

For relatively large values of $G^{(1)} / G^{(2)}$ and $G^{(3)} / G^{(2)}$ given by $G^{(1)} / G^{(2)}$ $=G^{(3)} / G^{(2)}=10$, the non-dimensionalized effective stiffness $\widehat{K}_{33 \mathrm{I}}$ is plotted against $\widehat{a}_{\mathrm{I}} / h$ in Figure 5.7 for $\rho_{\mathrm{I}}=\rho_{\mathrm{II}}=0.4,0.5$ and 0.6 . For a fixed value of $\rho_{\mathrm{I}}$, the effective stiffness increases as $\widehat{a}_{\mathrm{I}} / h$ increases. This is as expected, as the micro-cracks on the parallel planes $x_{2}=0$ and $x_{2}=h$ are more stable having smaller displacement jumps over the micro-cracks when the distance 
(a)

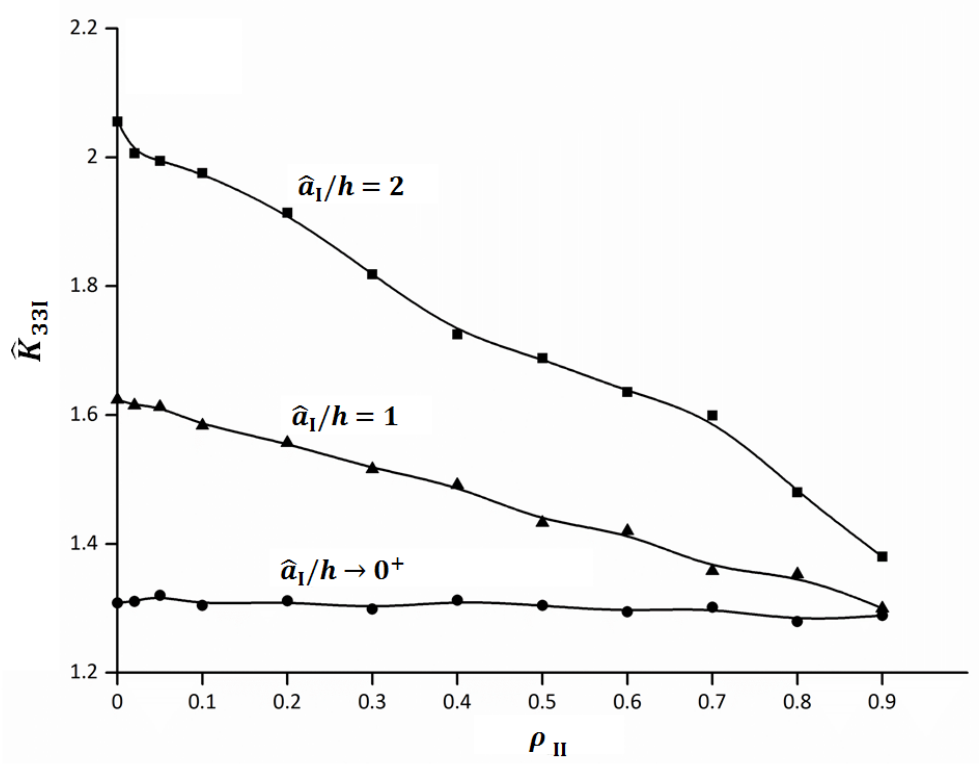

(b)

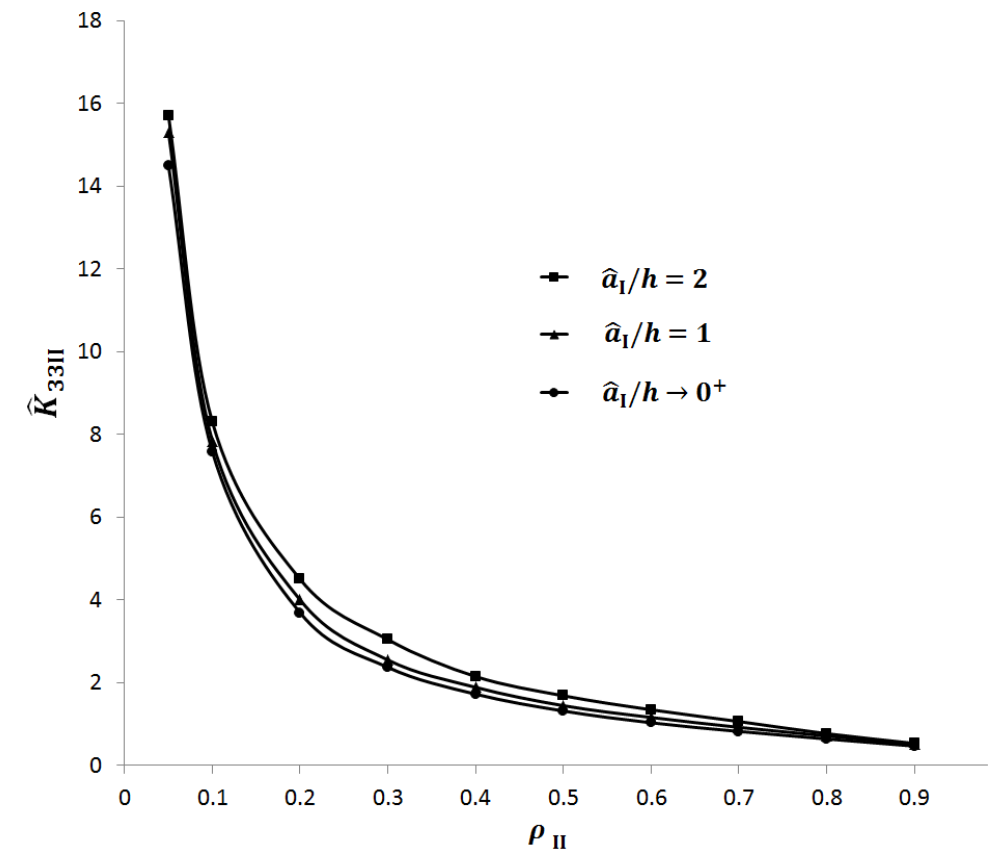

Figure 5.8: Plots of $\widehat{K}_{33 \mathrm{I}}$ and $\widehat{K}_{33 \mathrm{II}}$ against $\rho_{\mathrm{II}}$ for $\rho_{\mathrm{I}}=0.5, G^{(1)} / G^{(2)}$ $=G^{(3)} / G^{(2)}=10$ and selected values of $\widehat{a}_{\mathrm{I}} / h$. 
between the planes are smaller. The statistical simulations for other values of $\rho_{\mathrm{I}}=\rho_{\mathrm{II}}$ and $G^{(1)} / G^{(2)}=G^{(3)} / G^{(2)}$ within the range $0.1 \leq \rho_{\mathrm{I}} \leq 0.9$ and $0.5 \leq \log _{10}\left(G^{(1)} / G^{(2)}\right) \leq 3$ give the similar results.

Figure 5.7 also shows that the values of $\widehat{K}_{33 \mathrm{I}}$ tend to the corresponding effective stiffness calculated in Chapter 3 as $a_{\mathrm{I}} / h \rightarrow 0^{+}$for a fixed $\rho_{\mathrm{I}}$. Note that as $a_{\mathrm{I}} / h$ tends to $0^{+}$here each of the micro-cracked interfaces in Figure 5.1 may be regarded as an interface between two dissimilar elastic half-spaces.

For $\rho_{\mathrm{I}}=0.5$ and $G^{(1)} / G^{(2)}=G^{(3)} / G^{(2)}=10$, Figure 5.8 plots the nondimensionalized effective stiffness coefficients $\widehat{K}_{33 \mathrm{I}}$ and $\widehat{K}_{33 \mathrm{II}}$ against $\rho_{\text {II }}$ for selected values of $\widehat{a}_{\mathrm{I}} / h$. For a fixed value of $\widehat{a}_{\mathrm{I}} / h$, both $\widehat{K}_{33 \mathrm{I}}$ and $\widehat{K}_{33 \mathrm{II}}$ become smaller as $\rho_{\mathrm{II}}$ increases, as may be expected. This observation is not surprising as increasing the damage ratio on interface II has the effect of increasing the displacement jumps over the micro-cracks on both interfaces I and II. Note that for a fixed $\widehat{a}_{\mathrm{I}} / h$ the effective stiffness coefficient $\widehat{K}_{33 \mathrm{I}}$ tends to a certain finite value but $\widehat{K}_{33 I I}$ tends to infinity as $\rho_{\text {II }}$ approaches zero, that is, as interface II becomes more perfectly bonded like. For a fixed $\widehat{a}_{\mathrm{I}} / h, \widehat{K}_{33 \mathrm{II}}$ decreases more drastically than $\widehat{K}_{33 \mathrm{I}}$ as $\rho_{\text {II }}$ increases from 0.05 to 0.9 . Calculations using other values of $\rho_{\mathrm{I}}, \widehat{a}_{\mathrm{I}} / h$ and $G^{(1)} / G^{(2)}=G^{(3)} / G^{(2)}$ within the ranges $0.05 \leq \rho_{\mathrm{I}} \leq 0.9$, $0<\widehat{a}_{\mathrm{I}} / h \leq 2$ and $0.001 \leq G^{(1)} / G^{(2)} \leq 1000$ show similar observations.

\subsection{Numerical Results for Inplane Deforma- tions}

Numerical values of the effective stiffness coefficients $k_{i j \mathrm{I}}$ and $k_{i j \mathrm{II}}$ are obtained here for micro-cracked interfaces between particular orthotropic materials under an inplane deformation. The materials and their elastic moduli (in GPa) are given in Table 3.1. The specific cases are studied as follows. 


\subsubsection{A Perfect Interface and a Micro-cracked Interface}

For a special case, interface II is taken to be perfect (that is, $\rho_{\mathrm{II}}=0$ ) and interface I contains evenly distributed micro-cracks of equal length. The region where $0<x_{1}<L, x_{2}=0$ only contains one micro-crack $(M=1)$ of length $2 a_{\text {I }}$ and the tips $\left(a_{\mathrm{I}}^{(1)}, 0\right)$ and $\left(b_{\mathrm{I}}^{(1)}, 0\right)$ of the micro-crack are given by $(5.36)$. The materials in the layer and the lower half-space are carbon-epoxy and graphiteepoxy respectively. The elastic moduli (in GPa) of the material in the upper half-space are chosen to be

$$
C_{11}^{(1)}=136.2 \alpha, C_{22}^{(1)}=9.2 \alpha, C_{12}^{(1)}=3.9 \alpha, C_{66}^{(1)}=4.3 \alpha,
$$

where $\alpha$ is a positive constant.

If $\alpha$ is very small, the material in the upper half-space is extremely soft compared to the materials in the layer and the lower half-space. Thus, the limiting case in which $\alpha \rightarrow 0^{+}$gives a micro-cracked interface between an elastic half-space and a thin layer with one of its edges acted upon by prescribed tractions, as in Subsection 4.2.2.

If $\alpha=1$, the upper half-space and the layer are both occupied by carbonepoxy. Since interface II is perfect, $\alpha=1$ corresponds to the special case of a micro-cracked interface (given by interface I) between two dissimilar homogeneous elastic half-spaces. Such a special case can also be recovered from the analysis here for any other value of $\alpha$ by letting the width of the layer tend to infinity.

Since interface II is perfectly bonded, the displacement jumps $\Delta u_{j \mathrm{II}}^{(\mathrm{imp})}\left(x_{1}\right)$ over the micro-cracks on interface II reduce to zero. For selected values of $\alpha$, $a_{\mathrm{I}} / h$ and $\rho_{\mathrm{I}}$, the hypersingular boundary integro-differential equations (5.16), (5.17), (5.19), (5.20) for $M=1$ and $\Delta u_{j \mathrm{II}}^{(\mathrm{imp})}\left(x_{1}\right)=0$ together with (5.24) are solved by using the numerical procedures in Subsection 5.5.2 for the unknown 
functions $u_{r}^{(\mathrm{imp})}\left(x_{1}, h^{-} / 2\right), \sigma_{r 2}^{(\mathrm{imp})}\left(x_{1}, h^{-} / 2\right), u_{r}^{(\mathrm{imp})}\left(x_{1}, h^{+} / 2\right), \sigma_{r 2}^{(\mathrm{imp})}\left(x_{1}, h^{+} / 2\right)$ and $\Delta u_{j \mathrm{I}}^{(\mathrm{imp})}\left(x_{1}\right)$. Once the displacement jumps $\Delta u_{j \mathrm{I}}^{(\mathrm{imp})}\left(x_{1}\right)$ over interface I are obtained, the effective stiffness coefficients $k_{r j \mathrm{I}}$ may be calculated readily by using the first equation in (5.8) for two independent sets of loads. The estimation of $k_{r j 1}$ shows that the values of the effective stiffness coefficients $k_{12 \mathrm{I}}$ and $k_{21 \mathrm{I}}$ are observed to be insignificant compared to the values of either $k_{11 \mathrm{II}}$ or $k_{22 \mathrm{I}}$. The values of $\left|k_{12 \mathrm{I}} / k_{11 \mathrm{I}}\right|$ and $\left|k_{21 \mathrm{I}} / k_{22 \mathrm{I}}\right|$ are of the order $10^{-5}$ or smaller such that $k_{12 \mathrm{I}}$ and $k_{21 \mathrm{I}}$ are regarded approximately equal to zero here.

For $\rho_{\mathrm{I}}=0.5$, the non-dimensionalized effective stiffness coefficients $a_{\mathrm{I}} k_{11 \mathrm{I}} / C_{66}^{(2)}$ and $a_{\mathrm{I}} k_{22 \mathrm{I}} / C_{66}^{(2)}$ are plotted against $a_{\mathrm{I}} / h$ for selected values of $\alpha$. The graphs of $a_{\mathrm{I}} k_{11 \mathrm{I}} / C_{66}^{(2)}$ and $a_{\mathrm{I}} k_{22 \mathrm{I}} / C_{66}^{(2)}$ for the special case $\alpha \rightarrow 0^{+}$, obtained for the model in Subsection 4.2.2, are also given in Figure 5.9. It is obvious that the graphs of $a_{\mathrm{I}} k_{11 \mathrm{I}} / C_{66}^{(2)}$ and $a_{\mathrm{I}} k_{22 \mathrm{I}} / C_{66}^{(2)}$ for smaller values of $\alpha$ are closer to those for $\alpha \rightarrow 0^{+}$. This gives a check for the analysis here against the model in Figure 4.3 .

As expected, the values of $a_{\mathrm{I}} k_{11 \mathrm{I}} / C_{66}^{(2)}$ and $a_{\mathrm{I}} k_{22 \mathrm{I}} / C_{66}^{(2)}$ for $\alpha=1$ are constants independent of $a_{\mathrm{I}} / h$. As noted earlier on, the case $\alpha=1$ can also be recovered from the analysis here for other values of $\alpha$ by letting the width of the layer tend to infinity. This is why Figure 5.9 shows that the graphs of $a_{\mathrm{I}} k_{11 \mathrm{I}} / C_{66}^{(2)}$ and $a_{\mathrm{I}} k_{22 \mathrm{I}} / C_{66}^{(2)}$ converge to the constant values of the effective stiffness coefficients for $\alpha=1$ as $a_{\mathrm{I}} / h$ approaches $0^{+}$.

For a fixed $\alpha$, the non-dimensionalized effective stiffness coefficients $a_{\mathrm{I}} k_{11 \mathrm{I}} / C_{66}^{(2)}$ and $a_{\mathrm{I}} k_{22 \mathrm{I}} / C_{66}^{(2)}$ in Figure 5.9 are observed to decrease and increase as $a_{\mathrm{I}} / h$ increases for $\alpha<1$ and $\alpha>1$ respectively. This observation may be explained as follows. The perfectly bonded thin layer and the upper half-space may be considered as a "combined half-space". For $\alpha<1$, the upper half-space is softer than the thin layer. Thus, the "combined half-space" becomes softer as 
(a)

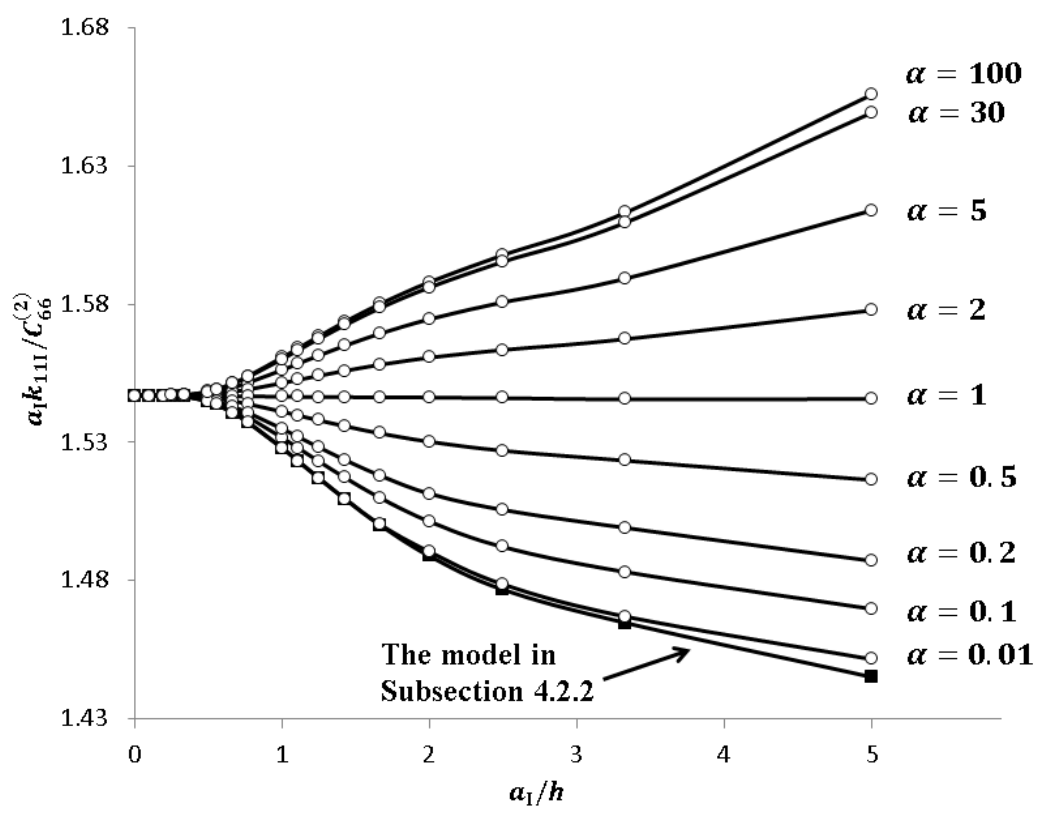

(b)

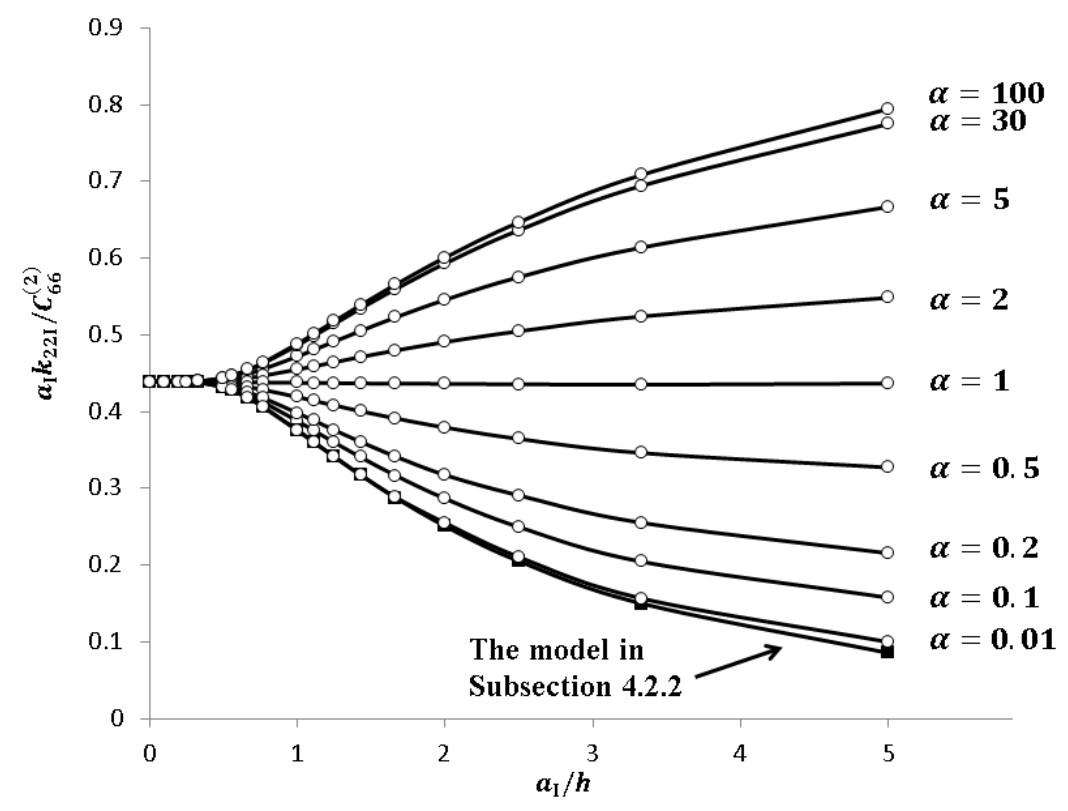

Figure 5.9: Plots of $a_{\mathrm{I}} k_{11 \mathrm{I}} / C_{66}^{(2)}$ and $a_{\mathrm{I}} k_{22 \mathrm{I}} / C_{66}^{(2)}$ against $a_{\mathrm{I}} / h$ for the cases where interface II is perfectly bonded with $\rho_{\mathrm{I}}=0.5$ and selected values of $\alpha$. 
(a)

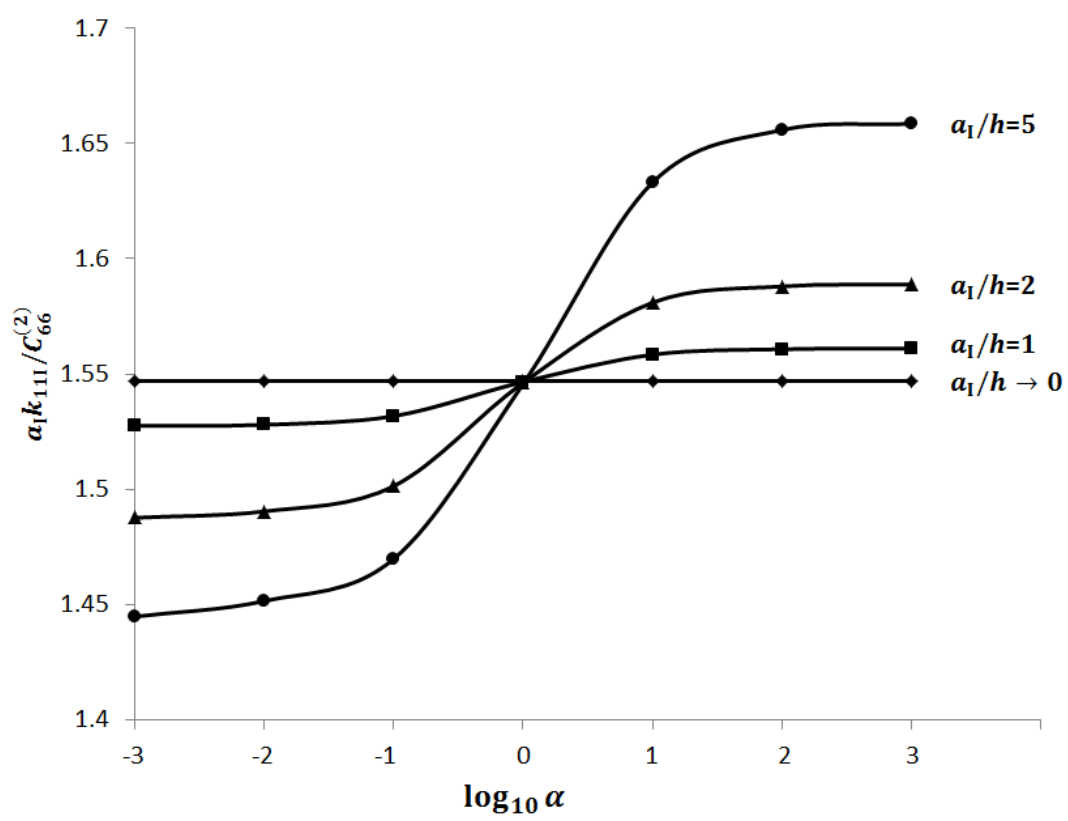

(b)

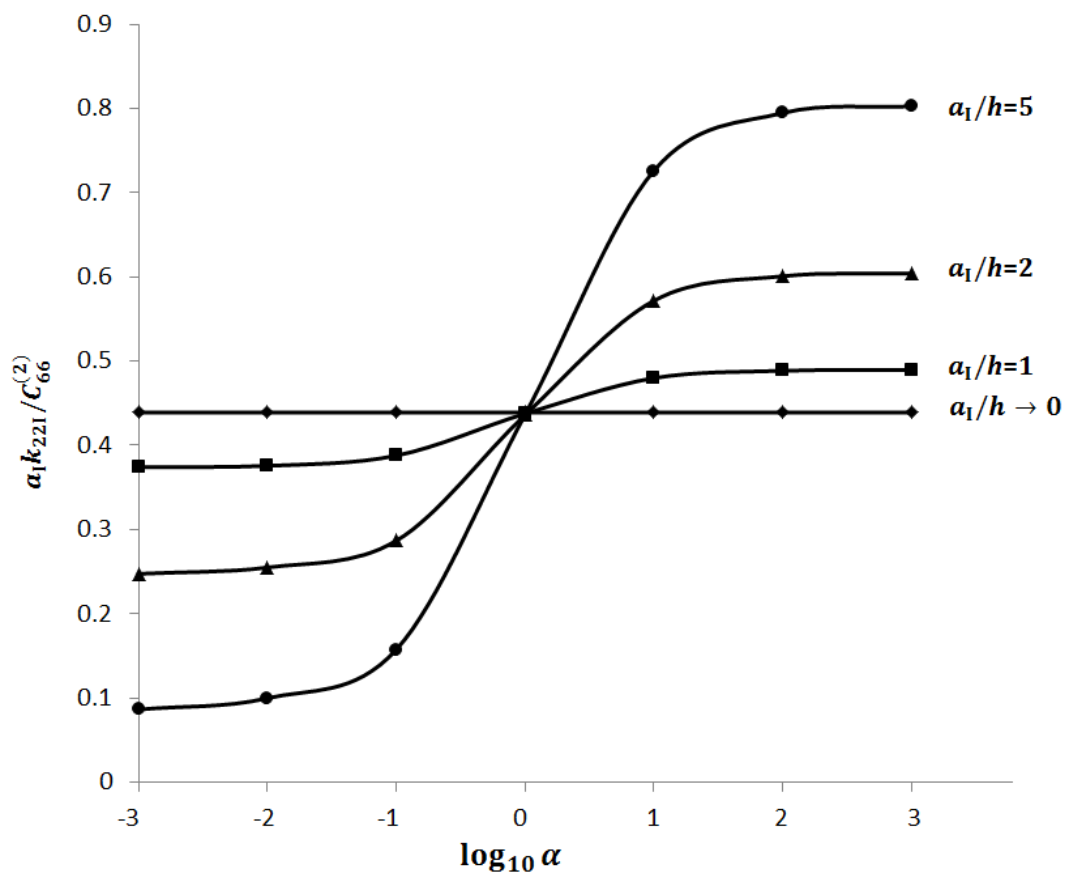

Figure 5.10: Plots of $a_{\mathrm{I}} k_{11 \mathrm{I}} / C_{66}^{(2)}$ and $a_{\mathrm{I}} k_{22 \mathrm{I}} / C_{66}^{(2)}$ against $\log _{10} \alpha$ for the cases where interface II is perfectly bonded with $\rho_{\mathrm{I}}=0.5$ and selected values of $a_{\mathrm{I}} / h$. 
the thin layer width decreases (that is, as $a_{\mathrm{I}} / h$ increases), giving larger average values of $\Delta u_{r \mathrm{I}}^{(\mathrm{imp})}\left(x_{1}\right)$ over interface I for larger $a_{\mathrm{I}} / h$. For $\alpha>1$, the upper halfspace is harder than the thin layer and hence the average values of $\Delta u_{r \mathrm{I}}^{(\mathrm{imp})}\left(x_{1}\right)$ over interface I are smaller for larger $a_{\mathrm{I}} / h$.

For a fixed value of $a_{\mathrm{I}} / h$, Figure 5.9 shows that $a_{\mathrm{I}} k_{11 \mathrm{I}} / C_{66}^{(2)}$ and $a_{\mathrm{I}} k_{22 \mathrm{I}} / C_{66}^{(2)}$ become larger as $\alpha$ increases. For the limiting case in which $\alpha \rightarrow \infty$, the layer is perfectly bonded to a rigid wall along the plane $x_{2}=h$ (so that $u_{1}=u_{2}=0$ on $\left.x_{2}=h\right)$. It may be of some interest to examine in detail how $a_{\mathrm{I}} k_{11 \mathrm{I}} / C_{66}^{(2)}$ and $a_{\mathrm{I}} k_{22 \mathrm{I}} / C_{66}^{(2)}$ vary with $\alpha$ for a fixed value of $a_{\mathrm{I}} / h$. For $\rho_{\mathrm{I}}=0.5$, Figure 5.10 plots $a_{\mathrm{I}} k_{111} / C_{66}^{(2)}$ and $a_{\mathrm{I}} k_{22 \mathrm{I}} / C_{66}^{(2)}$ against $\log _{10} \alpha$ for selected values of $a_{\mathrm{I}} / h$. From the graphs for a given $a_{\mathrm{I}} / h$, it is apparent that $a_{\mathrm{I}} k_{11 \mathrm{I}} / C_{66}^{(2)}$ and $a_{\mathrm{I}} k_{22 \mathrm{I}} / C_{66}^{(2)}$ tend to some constant values as $\alpha \rightarrow \infty$.

\subsubsection{A Particular Periodic Model for a Pair of Micro- cracked Interfaces}

The periodic model sketched in Figure 5.2 is used here for estimating the inplane effective stiffness coefficients for the pair of micro-cracked interfaces.

For selected materials in the thin layer and the two half-spaces and selected values of $\rho_{\mathrm{I}}, \rho_{\mathrm{II}}$ and $a_{\mathrm{I}} / h$, the hypersingular boundary integro-differential equations (5.16), (5.17), (5.19), (5.20) for $M=1$ together with (5.24) are solved by using the numerical procedures in Subsection 5.5.2 for the unknown functions $u_{r}^{(\mathrm{imp})}\left(x_{1}, h^{-} / 2\right), \sigma_{r 2}^{(\mathrm{imp})}\left(x_{1}, h^{-} / 2\right), u_{r}^{(\mathrm{imp})}\left(x_{1}, h^{+} / 2\right), \sigma_{r 2}^{(\mathrm{imp})}\left(x_{1}, h^{+} / 2\right)$, $\Delta u_{j \mathrm{I}}^{(\mathrm{imp})}\left(x_{1}\right)$ and $\Delta u_{j \mathrm{II}}^{(\mathrm{imp})}\left(x_{1}\right)$. Once the displacement jumps $\Delta u_{j \mathrm{I}}^{(\mathrm{imp})}\left(x_{1}\right)$ and $\Delta u_{j \mathrm{II}}^{(\mathrm{imp})}\left(x_{1}\right)$ are obtained, the effective stiffness coefficients $k_{i j \mathrm{I}}$ and $k_{i j \mathrm{II}}$ are calculated readily by using (5.8) for two independent sets of loads. The estimation of the effective stiffness coefficients for the periodic model shows that all the values of $\left|k_{12 \mathrm{I}} / k_{11 \mathrm{I}}\right|,\left|k_{21 \mathrm{I}} / k_{22 \mathrm{II}}\right|,\left|k_{12 \mathrm{II}} / k_{11 \mathrm{II}}\right|$ and $\left|k_{21 \mathrm{II}} / k_{22 \mathrm{II}}\right|$ are of the order 
$10^{-5}$ or smaller. Thus the values of $k_{12 \mathrm{I}}, k_{21 \mathrm{I}}, k_{12 \mathrm{II}}$ and $k_{21 \mathrm{II}}$ are approximately equal to zero for the periodic model here.

Some specific case studies using the periodic model are carried out below.

Case (a): The thin layer and the two half-spaces are occupied by the same orthotropic material. The material is taken to be graphite-epoxy. The interfaces are taken to be identical, so that $\rho_{\mathrm{I}}=\rho_{\mathrm{II}}=\rho$, hence $a_{\mathrm{I}}=a_{\mathrm{II}}=a$, where $a$ and $\rho$ are constants such that $\rho=2 a / L$. For such a case, the effective stiffness coefficients are such that $k_{11 \mathrm{I}}=k_{11 \mathrm{II}}=k_{1}$ and $k_{22 \mathrm{I}}=k_{22 \mathrm{II}}=k_{2}$.

In Figure 5.11, the non-dimensionalized effective stiffness coefficients $a k_{1} / C_{66}$ and $a k_{2} / C_{66}$ are plotted against $a / h$ for selected values of $\rho$. Note that $C_{66}=$ 7.5 GPa for graphite epoxy. For a given value of $\rho$, the non-dimensionalized effective stiffness coefficient $a k_{1} / C_{66}$ is observed to have a local minimum at a certain value of $a / h$. The value of $a / h$ where the local minimum of $a k_{1} / C_{66}$ occurs appears to increase with $\rho$.

Also, for a given $\rho$, the non-dimensionalized effective stiffness coefficient $a k_{2} / C_{66}$ appears to increase as $a / h$ increases. This may be explained by taking into consideration the crack shielding effect described in Gao et al. [37], that is, for two parallel cracks in a homogeneous elastic space under remote pure tensile load, the displacement jump $\Delta u_{2}^{(\mathrm{imp})}\left(x_{1}\right)$ of each of the cracks decreases as the cracks get closer to each other.

For a given value of $a / h$, it appears that $a k_{1} / C_{66}$ and $a k_{2} / C_{66}$ have larger values for smaller values of $\rho$. This is not surprising as the micro-cracks are more stable and have smaller displacement jumps if the micro-cracks have lower density on the interfaces. 
(a)

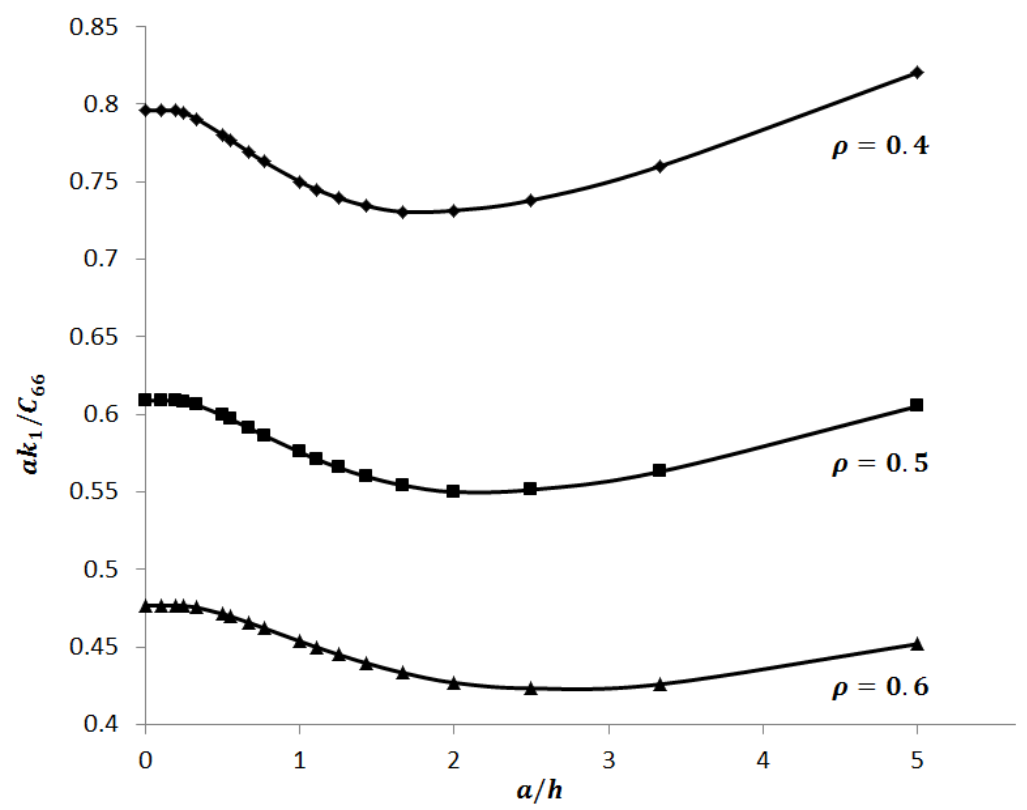

(b)

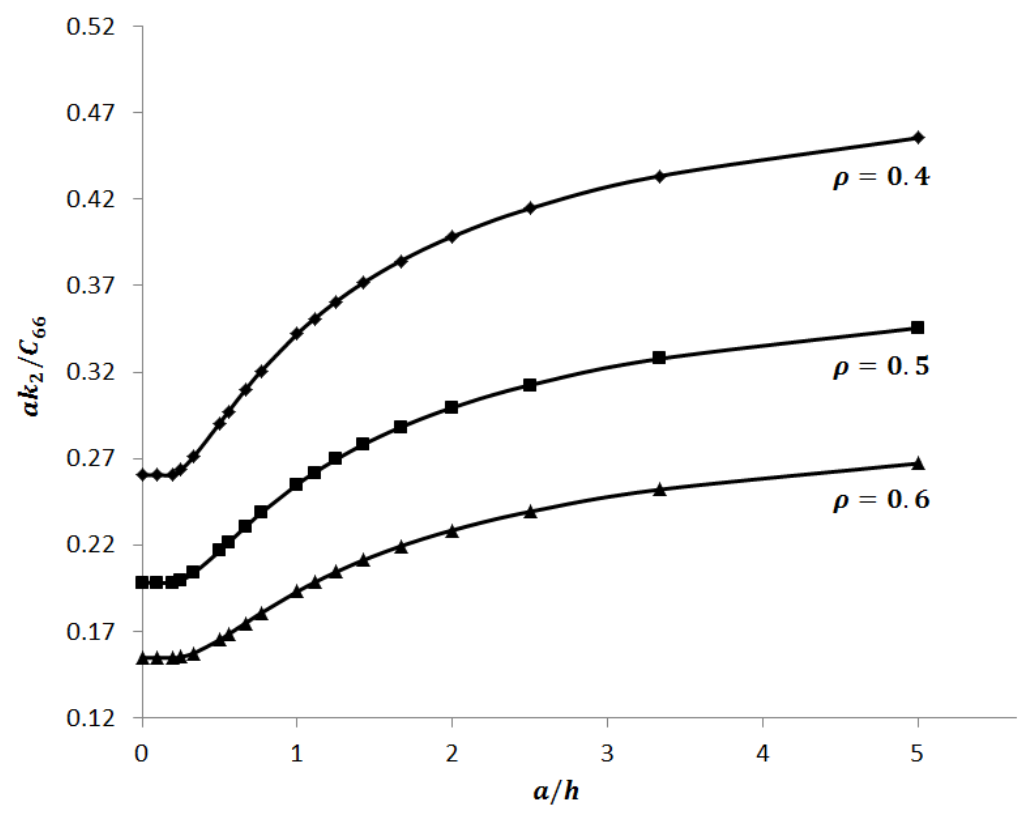

Figure 5.11: Plots of $a k_{1} / C_{66}$ and $a k_{2} / C_{66}$ against $a / h$ for $\rho=0.4,0.5$ and 0.6 . 
Case (b): As in Case (a), the layer and the half-spaces are occupied by graphite-epoxy. However, $\rho_{\mathrm{I}}$ and $\rho_{\mathrm{II}}$ are not necessarily equal here. For such a case, it may be more instructive to non-dimensionalize the effective stiffness coefficients as $\left(a_{\mathrm{I}}+a_{\mathrm{II}}\right) k_{r j \mathrm{I}} /\left(2 C_{66}\right)$ and $\left(a_{\mathrm{I}}+a_{\mathrm{II}}\right) k_{r j \mathrm{II}} /\left(2 C_{66}\right)$.

For $\rho_{\mathrm{I}}=0.5$, the non-dimensionalized effective stiffness coefficients $\left(a_{\mathrm{I}}+\right.$ $\left.a_{\mathrm{II}}\right) k_{r j \mathrm{I}} /\left(2 C_{66}\right)$ and $\left(a_{\mathrm{I}}+a_{\mathrm{II}}\right) k_{r j \mathrm{II}} /\left(2 C_{66}\right)$ are plotted against $\left(a_{\mathrm{I}}+a_{\mathrm{II}}\right) /(2 h)$ for selected values of $\rho_{\text {II }}$ in Figures 5.12 and 5.13 respectively. As expected, Figures 5.12 and 5.13 show that the graph of $\left(a_{\mathrm{I}}+a_{\mathrm{II}}\right) k_{r j \mathrm{I}} /\left(2 C_{66}\right)$ is the same as that of $\left(a_{\mathrm{I}}+a_{\mathrm{II}}\right) k_{r j \mathrm{II}} /\left(2 C_{66}\right)$ for $\rho_{\mathrm{II}}=0.5$ (that is, when interfaces I and II have the same damage ratio). For $\rho_{\mathrm{I}}=0.5$ and given values of $\rho_{\mathrm{II}}$ and $\left(a_{\mathrm{I}}+a_{\mathrm{II}}\right) /(2 h)$, Figures 5.12 and 5.13 show that $k_{r j \mathrm{I}}<k_{r j \mathrm{II}}$ for $\rho_{\text {II }}<0.5$ and $k_{r j \mathrm{I}}>k_{r j \mathrm{II}}$ for $\rho_{\mathrm{II}}>0.5$. This observation is as expected because of the following reason. For $\rho_{\text {II }}<0.5$, interface II is less severely damaged than interface I $\left(\rho_{\mathrm{I}}=0.5\right)$, hence $\left|\Delta u_{r \mathrm{II}}^{(\mathrm{imp})}\left(x_{1}\right)\right|$ may be expected to be less than $\left|\Delta u_{r \mathrm{I}}^{(\mathrm{imp})}\left(x_{1}\right)\right|$ to give $k_{r j \mathrm{II}}>k_{r j \mathrm{I}}$. Similarly, for $\rho_{\text {II }}>0.5$, with interface II more severely damaged, $\left|\Delta u_{r \mathrm{II}}^{(\mathrm{imp})}\left(x_{1}\right)\right|$ is greater than $\left|\Delta u_{r \mathrm{I}}^{(\mathrm{imp})}\left(x_{1}\right)\right|$, hence $k_{r j \mathrm{II}}<k_{r j \mathrm{I}}$.

In Figure 5.12, the range of values in which non-dimensionalized effective stiffness coefficients $\left(a_{\mathrm{I}}+a_{\mathrm{II}}\right) k_{11 \mathrm{I}} /\left(2 C_{66}\right)$ and $\left(a_{\mathrm{I}}+a_{\mathrm{II}}\right) k_{22 \mathrm{I}} /\left(2 C_{66}\right)$ (for interface I) can have over the interval $0<\left(a_{\mathrm{I}}+a_{\mathrm{II}}\right) /(2 h) \leq 5$ is narrower if $\rho_{\mathrm{II}}$ has a smaller value. Thus, one may expect $\left(a_{\mathrm{I}}+a_{\mathrm{II}}\right) k_{11 \mathrm{I}} /\left(2 C_{66}\right)$ and $\left(a_{\mathrm{I}}+\right.$ $\left.a_{\mathrm{II}}\right) k_{22 \mathrm{I}} /\left(2 C_{66}\right)$ to tend to parameters that are independent of $\left(a_{\mathrm{I}}+a_{\mathrm{II}}\right) /(2 h)$ as $\rho_{\mathrm{II}} \rightarrow 0$. This observation is consistent with the fact that the effective stiffness coefficients of interface II are independent of the width of the layer if interface II is perfect $\left(\rho_{\mathrm{II}}=0\right)$ and the layer and the upper half-space are occupied by the same material.

For $\rho_{\mathrm{I}}=0.5$ and a given value of $\left(a_{\mathrm{I}}+a_{\mathrm{II}}\right) /(2 h)$, Figure 5.13 shows that the values of $\left(a_{\mathrm{I}}+a_{\mathrm{II}}\right) k_{11 \mathrm{II}} /\left(2 C_{66}\right)$ and $\left(a_{\mathrm{I}}+a_{\mathrm{II}}\right) k_{22 \mathrm{II}} /\left(2 C_{66}\right)$ increase as $\rho_{\mathrm{II}}$ 
(a)

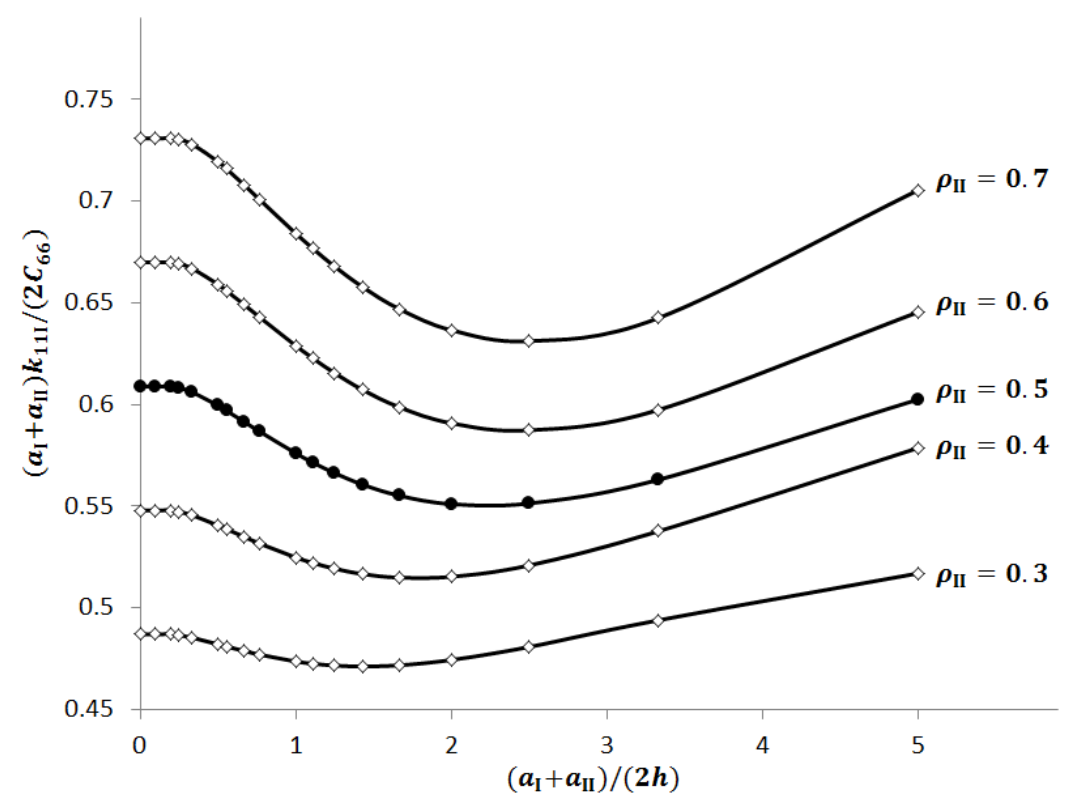

(b)

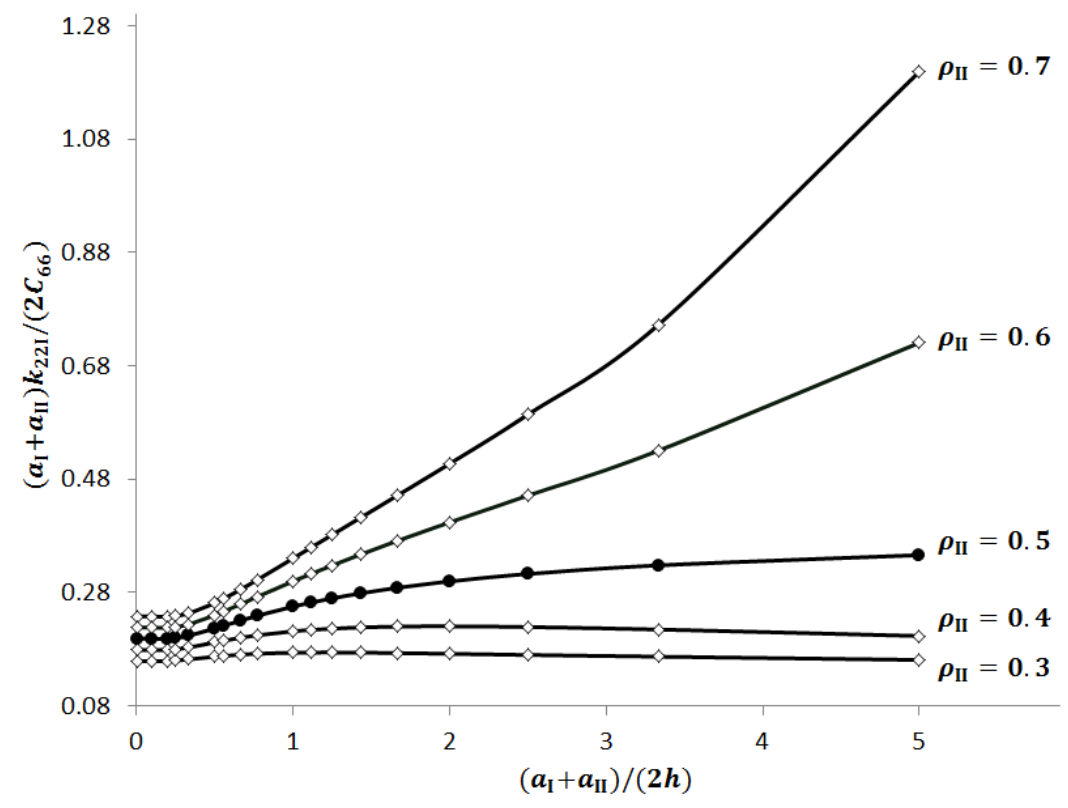

Figure 5.12: Plots of $\left(a_{\mathrm{I}}+a_{\mathrm{II}}\right) k_{11 \mathrm{I}} /\left(2 C_{66}\right)$ and $\left(a_{\mathrm{I}}+a_{\mathrm{II}}\right) k_{22 \mathrm{I}} /\left(2 C_{66}\right)$ against $\left(a_{\mathrm{I}}+a_{\mathrm{II}}\right) /(2 h)$ for selected values of $\rho_{\mathrm{II}}$. 
(a)

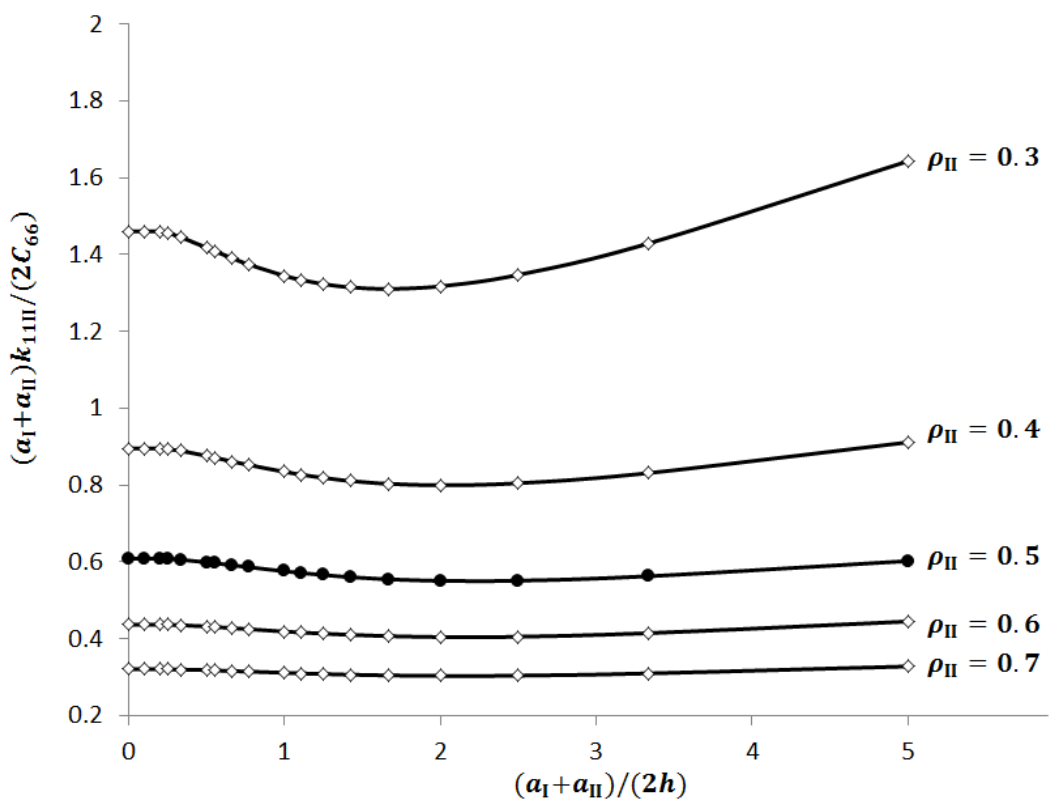

(b)

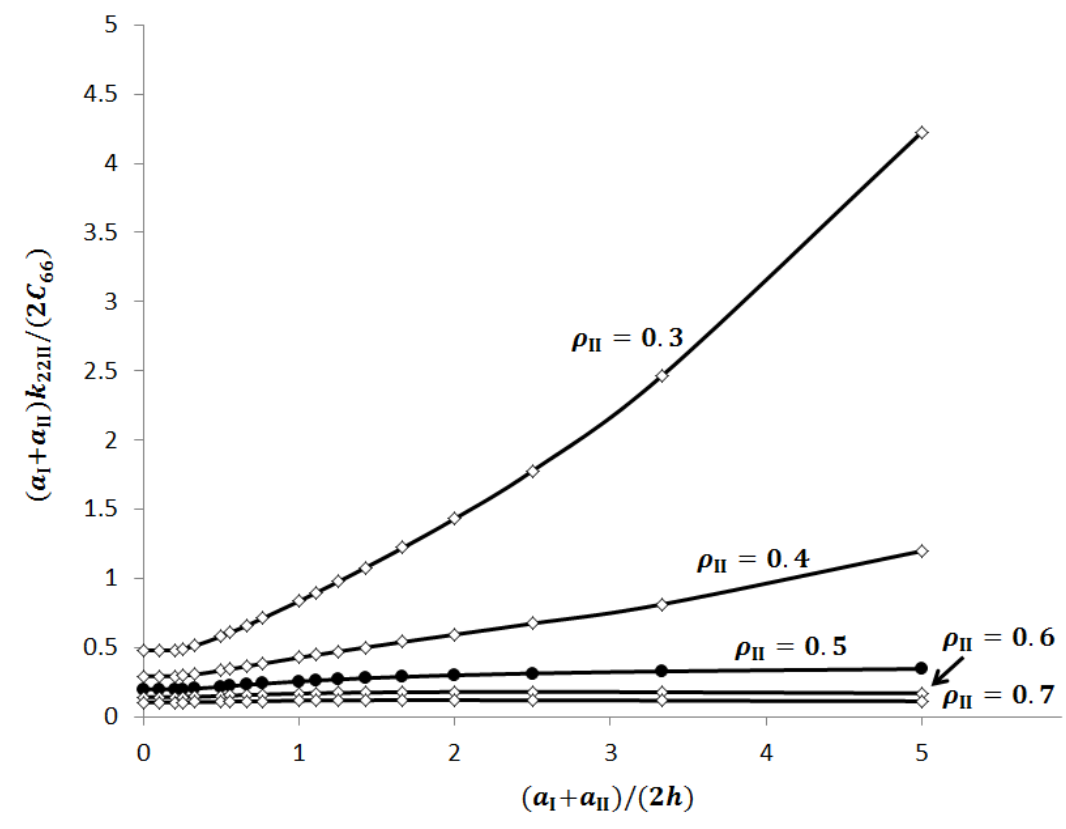

Figure 5.13: Plots of $\left(a_{\mathrm{I}}+a_{\mathrm{II}}\right) k_{11 \mathrm{II}} /\left(2 C_{66}\right)$ and $\left(a_{\mathrm{I}}+a_{\mathrm{II}}\right) k_{22 \mathrm{II}} /\left(2 C_{66}\right)$ against $\left(a_{\mathrm{I}}+a_{\mathrm{II}}\right) /(2 h)$ for selected values of $\rho_{\mathrm{II}}$. 


\section{Micromechanical Models for Weak Interfaces}

decreases to zero. This is as expected since both $k_{11 I I}$ and $k_{22 I I}$ should tend to infinity as $\rho_{\text {II }}$ tends to zero, that is as interface II approaches being perfect. From Figure 5.13 , it is also apparent that $\left(a_{\mathrm{I}}+a_{\mathrm{II}}\right) k_{22 \mathrm{II}} /\left(2 C_{66}\right)$ increases with $\left(a_{\mathrm{I}}+a_{\mathrm{II}}\right) /(2 h)$ at a faster rate for a smaller value of $\rho_{\mathrm{II}}$.

Case (c): The thin layer is occupied by graphite-epoxy. The material in the upper half-space is taken to be the same as that in the lower half-space. One of the following materials - martensite, graphite-epoxy and e-glass-epoxy - is selected to occupy the half-spaces. As in Case (a), the interfaces are such that $\rho_{\mathrm{I}}=\rho_{\mathrm{II}}=\rho$ and $a_{\mathrm{I}}=a_{\mathrm{II}}=a$, hence $k_{11 \mathrm{I}}=k_{11 \mathrm{II}}=k_{1}$ and $k_{22 \mathrm{I}}=k_{22 \mathrm{II}}=k_{2}$. Note that the values of $k_{12 \mathrm{I}}, k_{21 \mathrm{I}}, k_{12 \mathrm{II}}$ and $k_{21 \mathrm{II}}$ are considered approximately equal to zero.

For $\rho=0.5$, Figure 5.14 plots the non-dimensionalized effective stiffness coefficients $a k_{1} / C_{66}^{(2)}$ and $a k_{2} / C_{66}^{(2)}$ against $a / h$ for selected materials in the halfspaces. Note that the thin layer and the half-spaces are occupied by the same materials if the elastic moduli of graphite-epoxy are used for the half-spaces. Figure 5.14(a) shows that, as $a / h$ increases, $a k_{1} / C_{66}^{(2)}$ increases if the half-spaces are occupied by martensite, but $a k_{1} / C_{66}^{(2)}$ becomes smaller if the half-spaces are occupied by e-glass-epoxy. In Figure 5.14(b), the rate of increase in $a k_{2} / C_{66}^{(2)}$ with increasing $a / h$ is the most drastic when the half-spaces are occupied by martensite. For a given $a / h$, Figure 5.14 shows that the values of the effective coefficients for graphite-epoxy half-spaces are respectively smaller and larger than those for martensite and e-glass-epoxy half-spaces.

The observations above on the effective stiffness coefficients can be explained by noting the relative strength of the three materials selected for the analysis here. Martensite is the hardest of the three materials, whereas e-glassepoxy is the softest. Thus, when the half-spaces are occupied by martensite, 
(a)

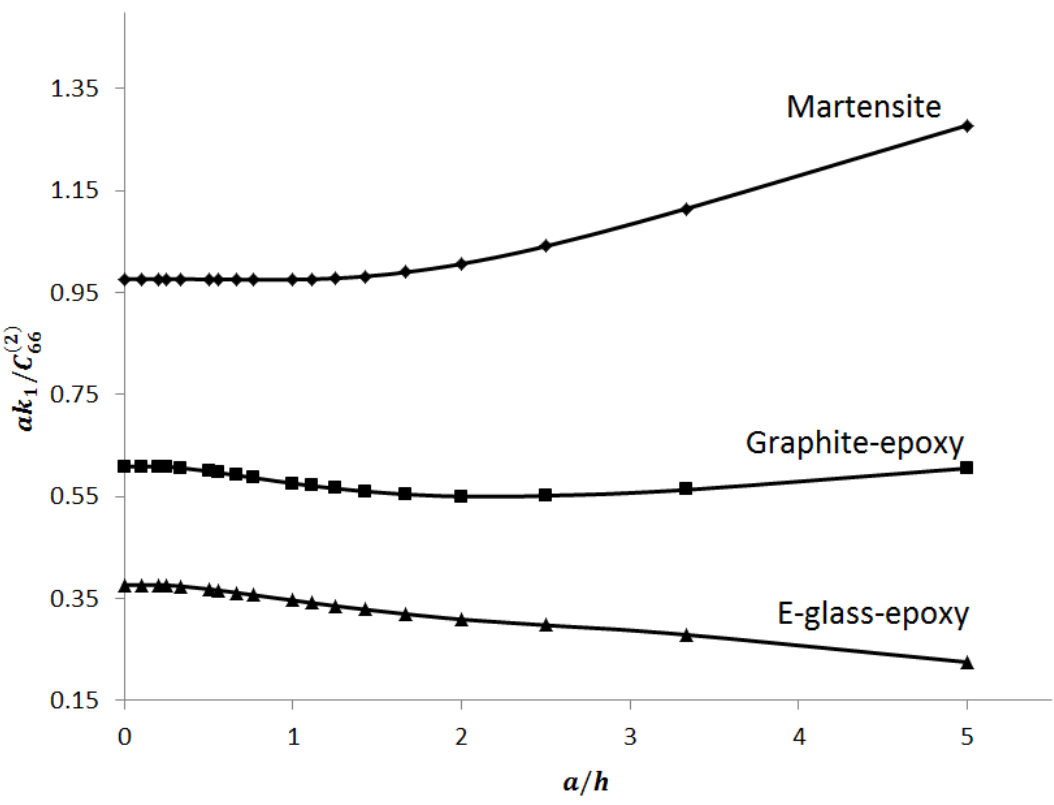

(b)

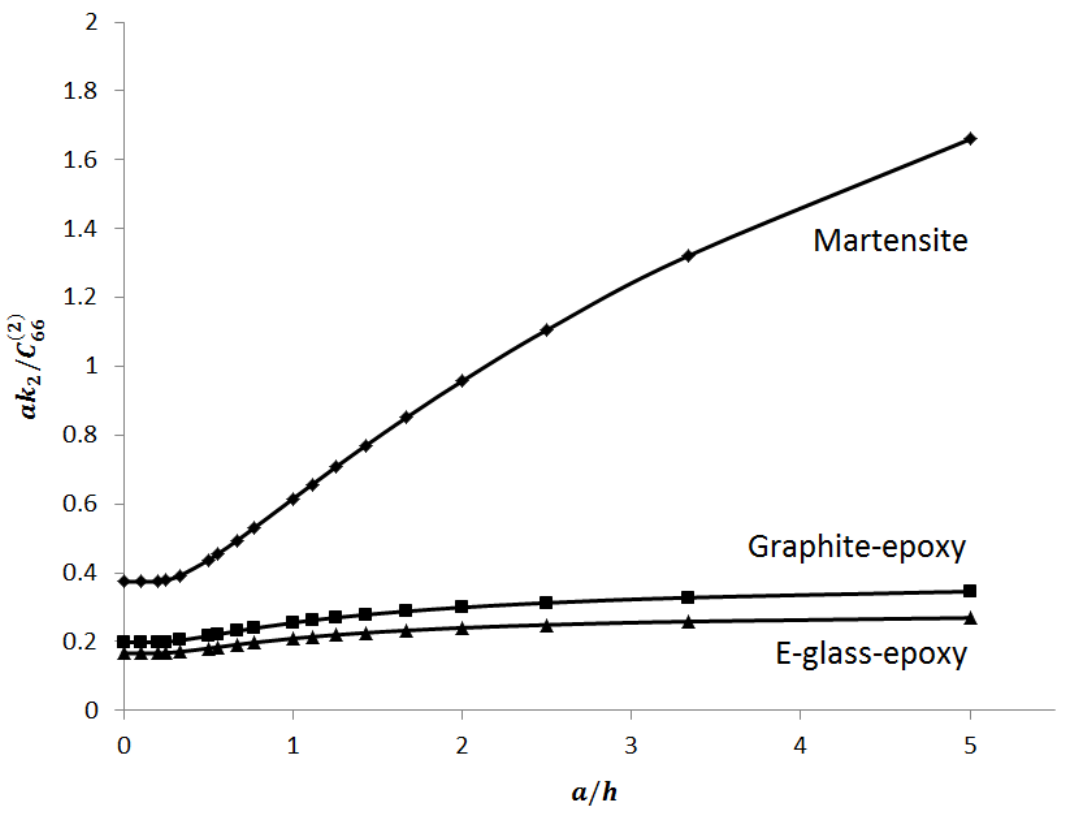

Figure 5.14: Plots of $a k_{1} / C_{66}^{(2)}$ and $a k_{2} / C_{66}^{(2)}$ against $a / h$ for the cases where martensite, graphite-epoxy and e-glass-epoxy are used as the material of the half-spaces. 
the trimaterial on the whole becomes "stronger" and the micro-cracks on the interfaces have relatively smaller displacement jumps over the micro-cracks, as $a / h$ increases (that is, as the width of the layer decreases). At the same time, as $a / h$ increases, the jump in the displacement $u_{2}$ over the micro-cracks also become smaller due to the shielding effect of parallel micro-cracks as explained in Case (a). All this combines to increase the effective stiffness coefficient $k_{2}$ of the interfaces drastically when the half-spaces are occupied by martensite. When the half-spaces are occupied by graphite-epoxy which is also the material in the layer, only the shielding effect of the parallel micro-cracks contributes to increasing the effective stiffness coefficient $k_{2}$ as $a / h$ increases. For e-glass-epoxy half-spaces, increasing $a / h$ has the effect of weakening the overall strength of the trimaterial, hence decreasing the effective stiffness coefficients. However, as shown in Figure 5.14(b) for e-glass-epoxy, the effective stiffness $k_{2}$ may still increase with increasing $a / h$ if the micro-crack shielding effect in increasing $k_{2}$ is more dominant than the opposing effect due to the weakening of the trimaterial.

\subsubsection{Micromechanical-statistical Simulations}

The micromechanical-statistical model as described in Subsection 5.6.2 is used here for estimating the effective stiffness coefficients of the interfaces. The thin layer is occupied by graphite-epoxy. Both the upper and lower half-spaces are taken to be occupied by e-glass-epoxy.

For selected values of $\rho_{\mathrm{I}}, \rho_{\mathrm{II}}$ and $\widehat{a}_{\mathrm{I}} / h, N$ pairs of randomly generated interfaces form a sample to generate the statistical data for the effective stiffness coefficients of interfaces I and II. For each pair of the interfaces, the nondimensionalized effective stiffness coefficients $\widehat{a}_{\mathrm{I}} k_{r j \mathrm{I}} / C_{66}^{(2)}$ and $\widehat{a}_{\mathrm{II}} k_{r j \mathrm{II}} / C_{66}^{(2)}\left(\widehat{a}_{\mathrm{I}}\right.$ and $\widehat{a}_{\mathrm{II}}$ are respectively the average half length of the micro-cracks on inter- 
faces I and II) are calculated as explained in Section 5.4. If $\widehat{a}_{\mathrm{I}} k_{r j \mathrm{I}} / C_{66}^{(2)}$ and $\widehat{a}_{\mathrm{II}} k_{r j \mathrm{II}} / C_{66}^{(2)}$ for the $n$-th interface are respectively written as $K_{i j \mathrm{I}}^{(n)}$ and $K_{i j \mathrm{II}}^{(n)}$, the mean values $\widehat{K}_{i j \mathrm{I}}, \widehat{K}_{i j \mathrm{II}}$ and the standard deviations $s_{i j \mathrm{I}}, s_{i j \mathrm{II}}$ of the nondimensionalized effective stiffness coefficients of interfaces I and II obtained from the $N$ pairs of randomly generated interfaces are calculated by

$$
\widehat{K}_{i j \mathrm{I}}=\frac{1}{N} \sum_{n=1}^{N} K_{i j \mathrm{I}}^{(n)} \text { and } s_{i j \mathrm{I}}=\sqrt{\frac{1}{N-1} \sum_{n=1}^{N}\left(K_{i j \mathrm{I}}^{(n)}-\widehat{K}_{i j \mathrm{I}}\right)^{2}},
$$

and

$$
\widehat{K}_{i j \mathrm{II}}=\frac{1}{N} \sum_{n=1}^{N} K_{i j \mathrm{II}}^{(n)} \text { and } s_{i j \mathrm{II}}=\sqrt{\frac{1}{N-1} \sum_{n=1}^{N}\left(K_{i j \mathrm{II}}^{(n)}-\widehat{K}_{i j \mathrm{II}}\right)^{2}} \text { for interface II. }
$$

Statistical simulations carried out here show that at least 40 micro-cracks on each interface are required to homogenize the effective stiffness coefficients of the interface. Thus the number of micro-cracks on a period length of each interface is taken to be 40 in the following simulations.

To investigate the effect of $\widehat{a}_{\mathrm{I}} / h$ on the mean values of $\widehat{K}_{i j \mathrm{I}}$ and $\widehat{K}_{i j \mathrm{II}}$ of the non-dimensionalized effective stiffness coefficients, we randomly generate 50 pairs of interfaces for selected values of $\rho_{\mathrm{I}}, \rho_{\mathrm{II}}$ and $\widehat{a}_{\mathrm{I}} / h$. For each interface, 40 micro-cracks are randomly generated over a period length of the interface - the lengths of the 40 micro-cracks follow $\chi^{2}(5)$ distribution and the microcracks are randomly distributed within the period length of the interface.

For $\rho_{\mathrm{I}}=\rho_{\mathrm{II}}=0.5$, Figure 5.15 plots the mean values $\widehat{K}_{i j \mathrm{I}}$ of the nondimensionalized effective stiffness coefficients against $\widehat{a}_{\mathrm{I}} / h$. The values of $\widehat{K}_{i j \mathrm{II}}$ are, as expected, very close to the values of $\widehat{K}_{i j \mathrm{I}}$ and therefore not plotted in 


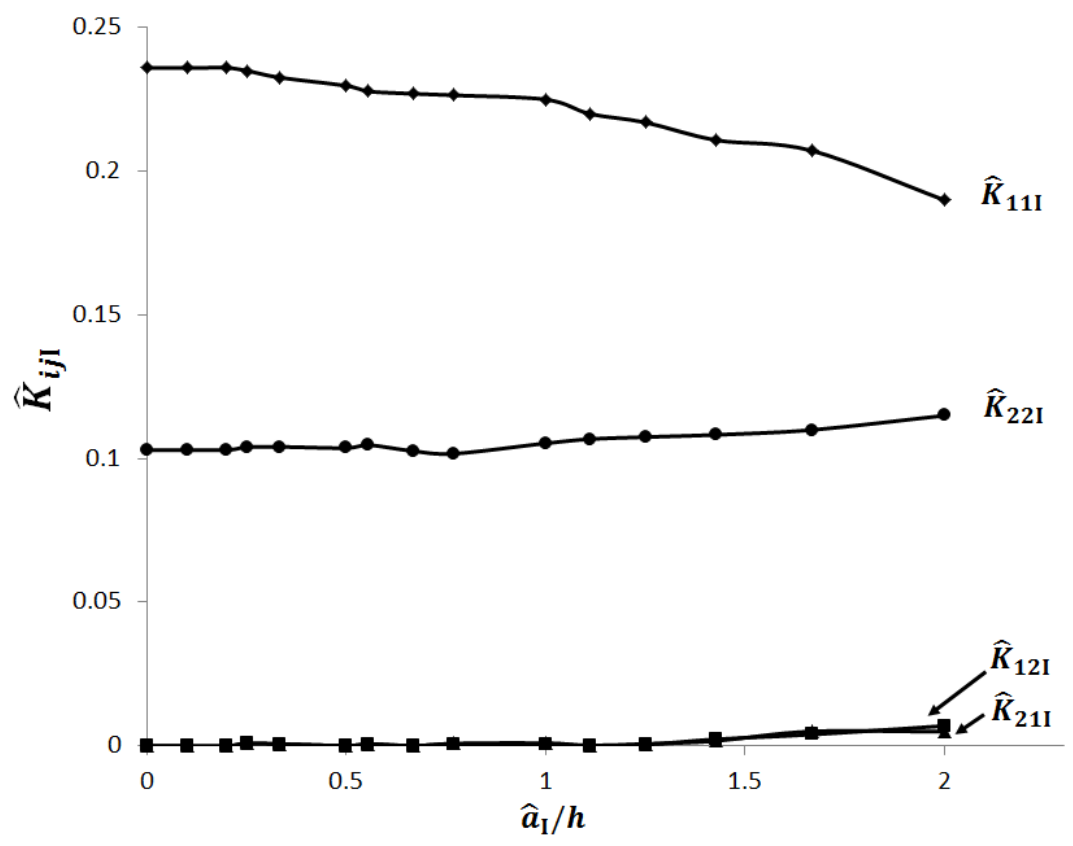

Figure 5.15: Plots of the mean values $\widehat{K}_{i j \text { I }}$ of the effective stiffness coefficients against $\widehat{a}_{\mathrm{I}} / h$ for $\rho_{\mathrm{I}}=\rho_{\mathrm{II}}=0.5$.

Figure 5.15. As $\widehat{a}_{\mathrm{I}} / h$ increases, $\widehat{K}_{11 \mathrm{I}}$ decreases but the value of $\widehat{K}_{22 \mathrm{I}}$ becomes larger. This observation shows the same trends with the corresponding plots of $a k_{1} / C_{66}^{(2)}$ and $a k_{2} / C_{66}^{(2)}$ against $\widehat{a}_{\mathrm{I}} / h$ in Figure 5.15 for the periodic model. Moreover, $\widehat{K}_{12 \mathrm{I}}$ and $\widehat{K}_{21 \mathrm{I}}$ are observed to be very small in magnitude compared to $\widehat{K}_{11 \mathrm{I}}$ and $\widehat{K}_{22 \mathrm{I}}$.

\subsection{Summary}

Hypersingular integro-differential equations are derived for micro-cracks lying on two parallel interfaces which separate a thin elastic layer from two elastic half-spaces. The equations are employed in micromechanical models for estimating the effective stiffness coefficients of the micro-cracked interfaces.

For antiplane deformations, the values of the effective stiffness coefficients 
computed for a periodic model where each of the interfaces has evenly distributed micro-cracks of equal length are compared with the corresponding values calculated by a three-phase model. The two sets of values show good agreement for wide ranges of parameters. The antiplane effective stiffness coefficients are also calculated for specific cases using a micromechanical-statistical model.

For inplane deformations, we have verified numerically that the case of a micro-cracked interface between an elastic half-space and a thin layer with one of its edges acted upon by prescribed tractions, as considered in Chapter 4, can be approximately recovered from the micromechanical model here by taking the upper interface of the trimaterial to be perfect and letting the upper half-space to be extremely soft. We have also used a particular special micromechanical model, where the equal length micro-cracks are evenly distributed on each of the interfaces, to study the effects of the thin layer width, the elastic constants of the trimaterial and interfacial micro-crack densities on the inplane effective stiffness coefficients of the pair of micro-cracked interfaces. In the special micromechanical model, some of the effective stiffness coefficients, namely $k_{12 \mathrm{I}}, k_{21 \mathrm{I}}, k_{12 \mathrm{II}}$ and $k_{21 \mathrm{II}}$, are zero. To check whether these coefficients are zero or not for interfaces where the micro-cracks have more general configurations, we have the micromechanical-statistical approach to generate the micro-cracks randomly and estimate the inplane effective stiffness coefficients. The coefficients $k_{12 \mathrm{I}}, k_{21 \mathrm{I}}, k_{12 \mathrm{II}}$ and $k_{21 \mathrm{II}}$ are not zero but they are numerically small in magnitude compared to the other coefficients $k_{12 \mathrm{I}}, k_{21 \mathrm{I}}, k_{12 \mathrm{II}}$ and $k_{21 \mathrm{II}}$. 



\section{Chapter 6}

\section{Research Contributions and Extensions}

\subsection{Summary of Contributions}

The contributions of this thesis are summarized as follows.

- Micromechanical models have been successfully implemented on the computer for estimating the effective stiffness coefficients of microscopically damaged interfaces between dissimilar orthotropic materials under antiplane and inplane deformations. The microscopically damaged interfaces are modeled as containing a periodic array of micro-cracks. Microcracked interfaces investigated here include: (i) an interface between two dissimilar half-spaces, (ii) an interface between a thin elastic layer and an elastic half-space, and (iii) a pair of parallel interfaces in an elastic trimaterial.

- Two highly simplified but useful micromechanical models, namely the three-phase model and the periodic model involving evenly spaced microcracks of equal length, are developed to gain quick initial insights into the various factors influencing the effective stiffness coefficients of a micro- 
cracked interface. The only micro details of the micro-cracked interface captured by these models are the micro-crack density (damage ratio of the interface) and the average micro-crack length. These models can also be used to verify each other's numerical procedures and provide qualitative check for the more realistic micromechanical-statistical model.

- In the micromechanical-statistical model, the random positions of the micro-cracks and the statistical variation of the micro-crack length are taken into consideration. Specifically, a period interval of the microcracked interface contains a selected number of randomly positioned micro-cracks with lengths that follow a $\chi^{2}$ distribution. According to our numerical investigations, 40 or more micro-cracks per period interval of the interface are required to homogenize the micro-cracked interface.

- With the aid of special Green's functions for perfect interfaces, all the micromechanical models are formulated in terms of hypersingular integrodifferential equations. One advantage of such formulations is that the displacement jumps across the damaged parts of the interface appear directly as unknown functions in the hypersingular integro-differential equations. The displacement jumps are needed in estimating the effective stiffness coefficients of the micro-cracked interface. Furthermore, the formulations here do not require the entire plane interface and the elastic space to be discretized into elements. This leads to relatively efficient calculations involving smaller number of unknowns.

- Numerical results giving useful insights into how the effective stiffness coefficients of micro-cracked interfaces are affected by various factors such as micro-crack density, the material constants and the geometries 
of the multilayered materials are obtained.

\subsection{Research Extensions}

Some possible extensions for the works presented in this thesis are listed below.

- The micromechanical models for micro-cracked interfaces may be extended to include three-dimensional interfacial micro-cracks under more general deformations.

- The micro-level analyses in Chapters 3,4 and 5 may be extended to estimate the effective properties of microscopically damaged interfaces between dissimilar multi-field materials (for example, piezoelectric materials) under antiplane or inplane deformations. Macro-level models for weak interfaces between multi-field materials may be found in Li and Lee $[60,61]$ and Wang and Pan [81].

- An attempt may be made to generalize the analysis in Chapter 5 to an arbitrary number of thin layers in multilayered materials. 



\section{Bibliography}

[1] Aboudi J, The generalized method of cells and high-fidelity generalized method of cells micromechanical models-A review, Mechanics of Advanced Materials and Structures 11 (2004) 329-366.

[2] Aboudi J, Mechanics of Composite Materials: A Unified Micromechanical Approach, Elsevier, New York, 2013.

[3] Aboudi J, Micromechanical analysis of composites by the method of cells-Update, Applied Mechanics Reviews 49 (1996) S83-S91.

[4] Aboudi J and Benveniste $\mathrm{Y}$, The effective moduli of cracked bodies in plane deformations, Engineering Fracture Mechanics 26 (1987) 171-184.

[5] Abramowitz M and Stegun I, Handbook of Mathematical Functions, Dover, New York, 1970.

[6] Afonso JC and Ranalli G, Elastic properties of three-phase composites: analytical model based on the modified shear-lag model and the method of cells, Composites Science and Technology 65 (2005) 1264-1275.

[7] Ang WT, A hypersingular-boundary integral equation method for a class of multiple interacting crack problems, PCCE 93-Proceedings of the 1st Pan-Pacific Conference on Computational Engineering (1993) 31-36. 
[8] Ang WT, A hypersingular-boundary integral equation method for cracks in an anisotropic elastic half-space with a partly irregular boundary, $B E M$ XIX-Proceedings of the 19th World Conference on the Boundary Element Method (1997) 339-347.

[9] Ang WT, Hypersingular Integral Equations in Fracture Analysis, Woodhead Publishing, Cambridge, 2013.

[10] Ang WT and Clements DL, Hypersingular integral equations for a thermoelastic problem of multiple planar cracks in an anisotropic medium, Engineering Analysis with Boundary Elements 23 (1999) 713-720.

[11] Ang WT, Clements DL and Cooke T, A hypersingular boundary integral equation for a class of antiplane crack problems for inhomogeneous elastic materials, Proceedings of the First UK Conference on Boundary Integral Methods (1997) 12-18.

[12] Ang WT and Fan H, A hypersingular boundary integral method for quasistatic antiplane deformations of an elastic bimaterial with an imperfect and visco-elastic interface, Engineering Computations 21 (2004) 529-539.

[13] Benveniste $\mathrm{Y}$ and Miloh T, Imperfect soft and stiff interfaces in twodimensional elasticity, Mechanics of Materials 33 (2001) 309-323.

[14] Berger J and Karageorghis A, The method of fundamental solutions for heat conduction in layered materials, International Journal for Numerical Methods in Engineering 45 (1999) 1681-1694.

[15] Berger H, Kurukuri S, Kari S, Gabbert U and Rodriguez-Ramos R, Numerical approaches for calculating the effective thermo-mechanical prop- 
erties of three-phase composites, Proceedings of The Seventh International Congress on Thermal Stresses TS2007 631-634.

[16] Berger JR and Tewary VK, Green's functions for boundary element analysis of anisotropic bimaterials, Engineering Analysis with Boundary Elements 25 (2001) 279-288.

[17] Bernardo JM, Algorithm AS 103: Psi (digamma) function, Journal of the Royal Statistical Society. Series C (Applied Statistics) 25 (1976) 315-317.

[18] Boström A and Golub M, Elastic SH wave propagation in a layered anisotropic plate with interface damage modelled by spring boundary conditions, Quarterly Journal of Mechanics and Applied Mathematics 62 (2009) 39-52.

[19] Boström A and Kvasha O, Elastic SH wave propagation in a layered anisotropic plate with periodic interface cracks: exact versus spring boundary conditions, Journal of Mechanics of Materials and Structures $\mathbf{5}$ (2010) 67-78.

[20] Chen YZ, Hypersingular integral equation approach for the multiple crack problem in an infinite plate, Acta Mechanica 108 (1995) 121-131.

[21] Chen YZ, Hypersingular integral equation method for three-dimensional crack problem in shear mode, Communications in Numerical Methods in Engineering 20 (2004) 441-454.

[22] Chen EL and Ang WT, Green's functions and boundary element analysis for bimaterials with soft and stiff planar interfaces under plane elastostatic deformations, Engineering Analysis with Boundary Elements 40 (2014) $50-61$. 
[23] Christensen RM, A critical evaluation for a class of micro-mechanics models, Journal of the Mechanics and Physics of Solids 38 (1990) 379-404.

[24] Christensen RM and Lo KH, Solutions for effective shear properties in three phase sphere and cylinder models, Journal of the Mechanics and Physics of Solids 27 (1979) 315-330.

[25] Clements DL, Boundary Value Problems Governed by Second Order Elliptic Systems, Pitman, 1981.

[26] Clements DL and Rizzo FJ, A method for the numerical solution of boundary value problems governed by second-order elliptic systems, Journal of Institute of Mathematics and Applications 22 (1978) 197-202

[27] Colombini D, Hassander H, Karlsson OJ and Maurer FHJ, Influence of the particle size and particle size ratio on the morphology and viscoelastic properties of bimodal hard/soft latex blends, Macromolecules 27 (2004) 6865-73.

[28] Colombini D and Maurer FHJ, Origin of additional mechanical transitions in multicomponent polymeric materials, Macromolecules 35 (2002) 5891902.

[29] Cui Z, Wang L, Jin AZ and Hong JS, Control of stress in multilayered MEMS devices, Proceedings of the 1st IEEE International Conference on Nano/Micro Engineered and Molecular Systems (2006) 1224-1227.

[30] Danielsson M, Parks DM and Boyce MC, Micromechanics, macromechanics and constitutive modeling of the elasto-viscoplastic deformation of rubber-toughened glassy polymers, Journal of the Mechanics and Physics of Solids $\mathbf{5 5}$ (2007) 533-561. 
[31] Fan H and Sze KY, A micro-mechanics model for imperfect interface in dielectric materials, Mechanics of Materials 33 (2001) 363-370.

[32] Fan H and Wang GF, Screw dislocation interacting with imperfect interface, Mechanics of Materials 35 (2003) 943-953.

[33] Fan H, Xie XM and Sze KY, Effective modulus of polycrystalline aggregates in different geometrical configurations, Materials Science and Engineering A $\mathbf{5 2 7}$ (2010) 5008-5017.

[34] Fannjiang AC, Paulino GH and Chan Y, Strain gradient elasticity for antiplane shear cracks: a hypersingular integrodifferential equation approach, SIAM Journal on Applied Mathematics 62 (2002) 1066-1091.

[35] Feng XQ, Mai YW and Qin QH, A micromechanical model for interpenetrating multiphase composites, Computational Materials Science $\mathbf{2 8}$ (2003) 486-493.

[36] Feng XQ, Tian Z, Liu YH and Yu SW, Effective elastic and plastic properties of interpenetrating multiphase composites, Applied Composite Materials 11 (2004) 33-55.

[37] Gao ZW, Lee ZW and Zhou YH, Crack tip shielding and anti-shielding effects of parallel cracks for a superconductor slab under an electromagnetic force, Journal of Mechanical Science and Technology 26 (2012) 353-357.

[38] García-Sánchez F, Zhang Ch, Sládek J and Sládek V, 2D transient dynamic crack analysis in piezoelectric solids by BEM, Computational Materials Science 39 (2007) 179-186. 
[39] Glodež S, Jezernik S, Kramberger J and Lassen T, Numerical modelling of fatigue crack initiation of martensitic steel, Advances in Engineering Software 41 (2010) 823-829.

[40] Guechaichia A and Trendafilova I, A simple frequency-based delamination detection and localization method without baseline model, Journal of Physics: Conference Series 382 (2012) 012033.

[41] Guinovart-Díaz R, Rodríguez-Ramosa R, Bravo-Castilleroa J, Sabinab FJ and Maugin GA, Closed-form thermoelastic moduli of a periodic threephase fiber-reinforced composite, Journal of Thermal Stresses 28 (2005) 1067-1093.

[42] Hamidi YK, Aktas L and Altan MC, Formation of microscopic voids in resin transfer molded composites, Journal of Engineering Materials and Technology 126 (2004) 420-426.

[43] Hashin Z, The spherical inclusion with imperfect interface, ASME Journal of Applied Mechanics 58 (1991) 444-449.

[44] Hills DA, Kelly PA, Dai DN and Korsunsky AM, Solution of Crack Problems: The Distributed Dislocation Technique, Kluwer Academic Publishers, Dordrecht, 1996.

[45] Ishii Y and Biwa S. Evaluation of interlayer interfacial stiffness and layer wave velocity of multilayered structures by ultrasonic spectroscopy, The Journal of the Acoustical Society of America 136 (2014) 183-191. 
[46] Ioakimidis NI, A new singular integral equation for the classical crack problem in plane and antiplane elasticity, International Journal of Fracture 21 (1983) 115-122.

[47] Ioakimidis NI, Application of Betti's reciprocal work theorem to the construction of the hypersingular integral equation of a plane crack in threedimensional elasticity, Journal of Elasticity 18 (1987) 165-171.

[48] Ioakimidis NI, The hypersingular integrodifferential equation of a straight crack along the interface of two bonded isotropic elastic half-spaces, International Journal of Fracture 38 (1988) R75-R79.

[49] Ju JW and Chen TM, Micromechanics and effective moduli of elastic composites containing randomly dispersed ellipsoidal inhomogeneities, Acta Mechanica 103 (1994) 103-121.

[50] Ju JW and Sun LZ, Effective elastoplastic behavior of metal matrix composites containing randomly located aligned spheroidal inhomogeneities, Part I: Micromechanics-based formulation, International Journal of Solids and Structures 38 (2001) 183-201.

[51] Kaya AC and Erdogan F, On the solution of integral equations with strongly singular kernels, The Quarterly of Applied Mathematics $\mathbf{4 5}$ (1987) 105-122.

[52] Kushch VI and Sevostianov I, Effective elastic properties of the particulate composite with transversely isotropic phases, International Journal of Solids and Structures 4 (2004) 885-906. 
[53] Long NMAN and Eshkuvatov ZK, Hypersingular integral equation for multiple curved cracks problem in plane elasticity, International Journal of Solids and Structures 46 (2009) 2611-2617.

[54] Li S and Wang G, Introduction to Micromechanics and Nanomechanics, World Scientific Publishing Company Incorporation, Hackensack, NJ, 2008.

[55] Linkov AM, Boundary Integral Equations in Elasticity Theory, Kluwer Academic Publishers, Dordrecht, 2002.

[56] Mandal BN and Chakrabarti A, Applied Singular Integral Equations, Science Publishers, Enfield, New Hampshire, 2011.

[57] Martin PA, End-point behaviour of solutions of hypersingular integral equations, Proceedings of the Royal Society A 432 (1991) 301-320.

[58] Michael A and Kwok CY, Design criteria for bi-stable behavior in a buckled multi-layered MEMS bridge, Journal of Micromechanics and Microengineering 16 (2006) 2034.

[59] Muralt P, Ferroelectric thin films for micro-sensors and actuators: a review, Journal of Micromechanics and Microengineering 10 (2000) 136146.

[60] Li YD and Lee KY, Crack tip shielding and anti-shielding effects of the imperfect interface in a layered piezoelectric sensor, International Journal of Solids and Structures 46 (2009) 1736-1742.

[61] Li YD and Lee KY, Interaction between an electrically permeable crack and the imperfect interface in a functionally graded piezoelectric sensor, International Journal of Engineering Science 47 (2009) 363-371. 


\section{Bibliography}

[62] Nagy PB, Ultrasonic classification of imperfect interfaces, Journal of Nondestructive Evaluation 11 (1992) 127-139.

[63] Nied HF, Periodic array of cracks in a half-plane subjected to arbitrary loading, Journal of Applied Mechanics 54 (1987) 642-648.

[64] Nix WD, Mechanical properties of thin films, Metallurgical Transactions A 20 (1989) 2217-2245.

[65] Pilarski A and Rose JL, A transverse-wave ultrasonic oblique incidence technique for interfacial weakness detection in adhesive bonds, Journal of Applied Physics 63 (1988) 300-307.

[66] Rizzo FJ, An integral equation approach to boundary value problems of classical elastostatics, Quarterly of Applied Mathematics 25 (1967) 83-95.

[67] Rokhlin SI and Huang W, Ultrasonic wave interaction with a thin anisotropic layer between two anisotropic solids: exact and asymptoticboundary condition methods, The Journal of the Acoustical Society of America 92 (1992) 1729-1742.

[68] Rokhlin SI and Wang YJ, Analysis of boundary conditions for elastic wave interaction with an interface between two solids, The Journal of the Acoustical Society of America 89 (1991) 503-515.

[69] Rubio-Gonzalez C and Mason JJ, Dynamic stress intensity factors at the tip of a uniformly loaded semi-infinite crack in an orthotropic material, Journal of the Mechanics and Physics of Solids 48 (2000) 899-925.

[70] Sadd MH, Elasticity - Theory, Applications and Numerics, Elsevier, 2005. 
[71] Schmauder S, Interfacial fracture in the presence of residual stresses, Fracture Mechanics of Ceramics 12 (1996) 443-450.

[72] Schmauder S and Meyer M, Correlation between Dundurs' parameters and elastic constants, Zeitschrift für Metallkunde 83 (1992) 524-527.

[73] Segurado J and LLorca J, Computational micromechanics of composites: the effect of particle spatial distribution, Mechanics of Materials 38 (2006) 873-883.

[74] Sertse $\mathrm{H}$ and $\mathrm{Yu} \mathrm{W}, \mathrm{A}$ Micromechanical Approach to Imperfect Interface Analysis of Heterogeneous Materials, Procedeeings of the 56th AIAA/ASCE/AHS/ASC Structures, Structural Dynamics, and Materials Conference, Florida, January, 2015.

[75] Stroh AN, Dislocations and cracks in anisotropic elasticity, Philosophical Magazine 3 (1958) 625-646.

[76] Sudak LJ, On the interaction between a dislocation and a circular inhomogeneity with imperfect interface in antiplane shear, Mechanics Research Communications 30 (2003) 53-59.

[77] Sudak LJ and Wang X, Green's function in plane anisotropic bimaterials with imperfect interface, IMA Journal of Applied Mathematics 71 (2006) 783-794.

[78] Sun C, Zhou ZF, Li WH, Huang QA, A simple method for extracting material parameters of multilayered MEMS structures using resonance frequency measurements, Journal of Micromechanics and Microengineering 24 (2014) 075014. 
[79] Xie XM and Fan H, Effective modulus of heterogeneous materials in thin film configurations, Materials Science and Engineering A 527 (2010) $5452-5461$.

[80] Wang X, Interaction between an edge dislocation and a circular inclusion with an inhomogeneously imperfect interface, Mechanics Research Communications 33 (2006) 17-25.

[81] Wang $\mathrm{X}$ and Pan E. A moving screw dislocation interacting with an imperfect piezoelectric bimaterial interface, physica status solidi 244 (2007) 1940-1956.

[82] Yu W and Tang T, Variational asymptotic method for unit cell homogenization of periodically heterogeneous materials, International Journal of Solids and Structures 44 (2007) 3738-3755.

[83] Yu W, Williams TO, Bednarcyk BA, Aboudi J and Tang T, A critical evaluation of the predictive capabilities of various advanced micromechanics models, Proceedings of the 48th AIAA/ASME/ASCE/AHS/AS Structures, Structural Dynamics and Materials Conference (2007) 23-26.

[84] Zhang Ch, Sládek J and Sládek V, Effects of material gradients on transient dynamic mode-III stress intensity factors in a FGM, International Journal of Solids and Structures 40 (2003) 5251-5270. 